\title{
Chemoselective $\alpha$-Sulfidation of Amides Using Sulfoxide Reagents
}

\author{
Mario Leypold, ${ }^{\ddagger}$ Kyan A. D’Angelo, ${ }^{\ddagger}$ and Mohammad Movassaghi* \\ Massachusetts Institute of Technology, Department of Chemistry, Cambridge, Massachusetts \\ 02139
}

$\$$ M.L. and K.A.D. contributed equally.

\section{Supporting Information}

\begin{tabular}{lr} 
General Procedures & S3 \\
\hline
\end{tabular}

\begin{tabular}{ll} 
Materials & S3 \\
\hline
\end{tabular}

$\begin{array}{ll}\text { Instrumentation } & \text { S3 }\end{array}$

Representative Procedure I for the Oxidation of Sulfides with $m$-CPBA $\quad$ S5

\begin{tabular}{ll} 
tert-Butyl Methyl Sulfoxide (2b) & S5 \\
\hline
\end{tabular}

Representative Procedure II for the Alkylation of tert-Butyl Methyl Sulfoxide (2b) $\quad$ S6

tert-Butyl Ethyl Sulfoxide (2c)

S6

tert-Butyl 3-Butenyl Sulfoxide (2d)

S7

\begin{tabular}{ll} 
tert-Butyl 2-Phenethyl Sulfoxide (2e) & S8 \\
\hline
\end{tabular}

\begin{tabular}{ll} 
Methyl 2-Phenethyl Sulfoxide (2f) & S9 \\
\hline
\end{tabular}

4-Methoxyphenmethyl Methyl Sulfoxide (2g) $\quad$ S10

\begin{tabular}{ll} 
Triphenylmethyl Methyl Sulfoxide (2h) & S11 \\
\hline
\end{tabular}

Screening of Reaction Conditions for the Formation of Sulfonium

\begin{tabular}{lr} 
Trifluoromethanesulfonate 3ba & S12 \\
\hline
\end{tabular}

\begin{tabular}{ll} 
Screening of Sulfoxides for the Spontaneous Sulfide Formation & S13 \\
\hline
\end{tabular}

Screening of Reaction Conditions for the Formation of Sulfonium

Trifluoromethanesulfonate 3ta

Method A: Representative Procedure for the $\alpha$-Sulfidation of Benzylic Amides with

Methyl Sulfoxides

Method B: Representative Procedure for the $\alpha$-Sulfidation of Benzylic Amides with tert-Butyl Sulfoxides

$\alpha$-SMe-Morpholine Amide 4a

$\alpha$-SMe-Morpholine Amide 4a (5.00 mmol scale)

Morpholine Amide Dimethyl Sulfonium Trifluoromethanesulfonate 3aa $\quad$ S18

\begin{tabular}{ll}
$\alpha$-SMe-Pyrrolidine Amide 4b & S19 \\
\hline
\end{tabular}

Pyrrolidine Amide Dimethyl Sulfonium Trifluoromethanesulfonate 3ba $\quad$ S20

$\begin{array}{ll}\alpha \text {-SMe-Weinreb Amide 4c } & \text { S21 }\end{array}$

\begin{tabular}{ll}
$\alpha-S M e-N$-Benzyl- $N$-Methyl Amide 4d & S22 \\
\hline
\end{tabular}

\begin{tabular}{ll}
$\alpha$-SMe- $N$-Phenyl- $N$-Methyl Amide 4e & S24 \\
\hline
\end{tabular}

\begin{tabular}{ll}
$\alpha$-SMe- $N$-Phenyl- $N$-Methyl Amide 4f & S25 \\
\hline
\end{tabular}

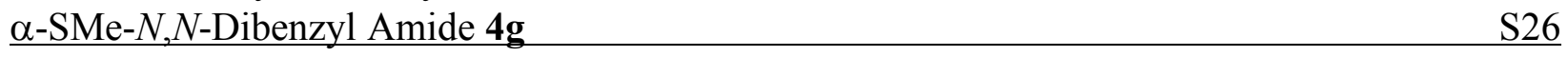

\begin{tabular}{ll}
$\alpha$-SMe-Morpholine Amide 4h & S28 \\
\hline
\end{tabular}

\begin{tabular}{lr}
$\alpha$-SMe-Morpholine Amide 4i & S30 \\
\hline
\end{tabular}

\begin{tabular}{ll}
$\alpha$-SMe-Morpholine Amide 4j & S31 \\
\hline
\end{tabular} 
$\alpha$-SMe-Morpholine Amide 4k

$\mathrm{S} 32$

$\alpha$-SMe-Morpholine Amide 41

$\alpha$-SMe-Morpholine Amide 4m

a-SMe-Morpholine Amide 4n

$\alpha$-SMe-Morpholine Amide 40

$\alpha$-SEt-Morpholine Amide 4p

$\alpha$-S(But-3-en-1-yl)-Morpholine Amide 4q

Method C: Representative Procedure for the $\alpha$-Sulfidation of Aliphatic Amides with

Methyl Sulfoxides

$\alpha$-SMe-Morpholine Amide 4s

$\alpha$-SMe-Morpholine Amide 4s $(5.00 \mathrm{mmol} \mathrm{scale})$

$\alpha$-SMe-Pyrrolidine Amide 4t

$\alpha$-SMe-Morpholine Amide 4u

$\underline{\alpha-S M e-M o r p h o l i n e ~ A m i d e ~ 4 v ~}$

$\alpha$-SMe-Morpholine Amide 4w

$\alpha$-SMe-Morpholine Amide 4x

S48

$\alpha$-SMe-Morpholine Amide 4y

$\alpha$-SMe- $N, N$-Dimethyl Amide $\mathbf{4 z}$

$N, N$-Dimethyl Amide Dimethyl Sulfonium Trifluoromethanesulfonate 3za

$\alpha$-S(2-Phenethyl)-Morpholine Amide 4aa

$\alpha$-SMe-Pyrrolidine Amide S4

S53

Overview of In Situ IR Experiments $\quad$ S54

In Situ IR Experiments

Overview of Mechanistic Experiments

Dimethyl Sulfoxide- ${ }^{18} \mathrm{O}-d_{6}\left(\mathbf{2 a -}{ }^{18} \boldsymbol{O}-\mathbf{d} \mathbf{6}\right)$

Mechanistic Experiments

X-Ray Diffraction Data of $N, N$-Dimethyl Amide Dimethyl Sulfonium

Trifluoromethanesulfonate 3za

Computational Information

Computed Reaction Profiles for the $\alpha$-Sulfidation of Benzylic Amides

$\mathrm{S} 74$

Optimized Cartesian Coordinates

References and Comments 
General Procedures. All reactions were performed in flame-dried round-bottom flasks, unless noted otherwise. The flasks were fitted with rubber septa, and reactions were conducted under a positive pressure of argon. Cannulae or gas-tight syringes with stainless steel needles were used to transfer air- or moisture-sensitive liquids or reagent solutions. Flash column chromatography was performed as described by Still et al. ${ }^{1}$ using granular silica gel (60 $\AA$ pore size, $40-63 \mu \mathrm{m}, 4-6 \%$ $\mathrm{H}_{2} \mathrm{O}$ content, Zeochem). Analytical thin layer chromatography (TLC) was performed using glass plates pre-coated with $0.25 \mathrm{~mm} \mathrm{230-400} \mathrm{mesh} \mathrm{silica} \mathrm{gel} \mathrm{impregnated} \mathrm{with} \mathrm{a} \mathrm{fluorescent} \mathrm{indicator}$ (254 nm, EMD Millipore 105715). TLC plates were visualized by exposure to short wave ultraviolet light $(254 \mathrm{~nm})$, and were irreversibly stained by treatment with an aqueous solution of ceric ammonium molybdate $(\mathrm{CAM})$ or an aqueous solution of potassium permanganate $\left(\mathrm{KMnO}_{4}\right)$ followed by heating $(\sim 1 \mathrm{~min})$ on a hot plate $\left(\sim 250^{\circ} \mathrm{C}\right)$. Organic solutions were concentrated at $29-$ $35{ }^{\circ} \mathrm{C}$ on rotary evaporators capable of achieving a minimum pressure of $\sim 2$ Torr.

Materials. Commercial reagents and solvents were used as received with the following exceptions: dichloromethane, tetrahydrofuran, and diethyl ether were purchased from J. T. Baker (CycletainerTM), and were purified by the method of Grubbs et al. under positive argon pressure. ${ }^{2}$ Benzene, chloroform- $d$, and all described pyridine bases were dried by distillation over calcium hydride or calcium chloride under an inert nitrogen or argon atmosphere, and were used directly. Trifluoromethanesulfonic anhydride and trifluoroacetic anhydride were dried by distillation over phosphorous pentoxide under an inert argon atmosphere. All other solvents and chemicals were purchased from Sigma-Aldrich, Alfa Aesar, Acros Organics, or Oakwood Chemicals.

Instrumentation. Proton nuclear magnetic resonance ( ${ }^{1} \mathrm{H}$ NMR) spectra were recorded with a Bruker AVANCE III 400 or Varian Unity Inova 500 spectrometer. Chemical shifts are recorded in parts per million on the $\delta$ scale, and are referenced from the residual protium in the NMR solvent $\left(\mathrm{CHCl}_{3}: \delta 7.26, \mathrm{CD}_{2} \mathrm{Cl}_{2}: \delta 5.32\right) .{ }^{3}$ Data are reported as follows: chemical shift [multiplicity $(\mathrm{s}=$ singlet, $\mathrm{d}=$ doublet, $\mathrm{t}=$ triplet, app-p = apparent pentet, app-sept $=$ apparent septet, $\mathrm{m}=$ multiplet, $\mathrm{bs}=$ broad singlet, $\mathrm{dd}=$ doublet of a doublet, $\mathrm{dt}=$ doublet of a triplet, $\mathrm{ddt}=$ doublet of a doublet of a triplet), coupling constant(s) in Hertz, integration]. Carbon-13 nuclear magnetic resonance $\left({ }^{13} \mathrm{C}\right.$ NMR) spectra were recorded with the same Bruker AVANCE III 400 or Varian Unity Inova 500 spectrometer, are recorded in parts per million on the $\delta$ scale, and are referenced from the carbon resonances of the solvent $\left(\mathrm{CDCl}_{3}: \delta 77.16, \mathrm{CD}_{2} \mathrm{Cl}_{2}: \delta 54.00\right)$. Data are reported as follows: chemical shift [multiplicity $(\mathrm{q}=$ quartet), coupling constant(s) in Hertz]. Fluorine-19 nuclear magnetic resonance $\left({ }^{19} \mathrm{~F}\right.$ NMR) spectra were recorded with a Bruker AVANCE III 400 spectrometer, are recorded in parts per million on the $\delta$ scale, and are referenced from the fluorine resonances of $\alpha, \alpha, \alpha$-trifluorotoluene $\left(\mathrm{C}_{6} \mathrm{H}_{5} \mathrm{CF}_{3}\right.$ in $\left.\mathrm{CDCl}_{3}: \delta-63.72\right)$. Infrared data were obtained with a Bruker ALPHA II Platinum-ATR spectrometer with a diamond probe, and are reported as follows: [frequency of absorption $\left(\mathrm{cm}^{-1}\right)$, intensity of absorption $(\mathrm{s}=$ strong, $\mathrm{m}=$ medium, $\mathrm{w}=$ weak, $\mathrm{br}=$ broad)]. React-IR experiments were recorded on an ASI React-IR 1000 instrument with a silicon probe. We thank Dr. Mohanraja Kumar at the Massachusetts Institute of Technology Department of Chemistry Instrumentation Facility for obtaining mass spectrometric data. Q-TOF mass analyses were performed on a Waters Q-TOF micro mass spectrometer using an electrospray $(\mathrm{ESI})(\mathrm{m} / \mathrm{z})$ ionization source in the positive and negative mode connected to a time of flight (TOF) mass detector. High resolution mass spectra (HRMS) were recorded on a JEOL ionSense DART AccuTOF 4G LC-plus using a direct analysis in real time (DART) ionization source. HRMS data are reported as $[\mathrm{M}+\mathrm{H}]^{+}$or $[\mathrm{M}]^{+}$for DART-TOF+ and ESI-TOF+ analyses. Chiral HPLC analysis 
was performed on an Agilent 1100 Series HPLC with a Chiralcel OD-H column as well as a Chiralpak AS-H column. Single crystal X-ray diffraction was carried out with the assistance of Dr. Peter Müller at the X-ray Diffraction Facility of the Massachusetts Institue of Technology Department of Chemistry using a Bruker X8 Kappa DUO four-circle diffractometer equipped with a Bruker APEX2 CCD. ${ }^{4}$ 


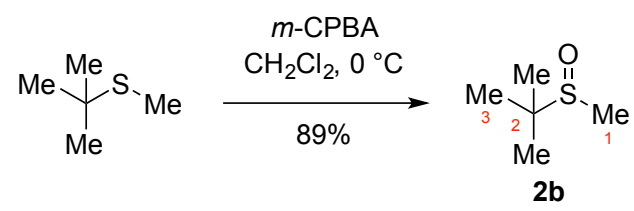

Representative Procedure I for the Oxidation of Sulfides with m-CPBA. tert-Butyl Methyl Sulfoxide (2b): $:^{5}$

$m$-Chloroperbenzoic acid ( $77 \mathrm{wt} \%, 9.0 \mathrm{~g}, 40 \mathrm{mmol}, 1$ equiv) was added in five portions over $10 \mathrm{~min}$ to a cooled solution of tert-butyl methyl sulfide ( $4.59 \mathrm{~g}, 44.0 \mathrm{mmol}, 1.1$ equiv) in dichloromethane $(120 \mathrm{~mL})$. After stirring the colorless suspension at $0{ }^{\circ} \mathrm{C}$ for $1 \mathrm{~h}$, a saturated aqueous solution of sodium bicarbonate $(120 \mathrm{~mL})$ was added, and the colorless biphasic mixture was stirred at $22{ }^{\circ} \mathrm{C}$ for $30 \mathrm{~min}$. The phases were separated, and the aqueous layer was extracted with dichloromethane $(15 \times 30 \mathrm{~mL})$. The combined colorless organic extracts were dried over sodium sulfate, were filtered, and were concentrated under reduced pressure. The light yellow crude material was purified via flash column chromatography on silica gel (eluent: dichloromethane $\rightarrow 4 \%$ methanol in dichloromethane) to yield sulfoxide $\mathbf{2 b}(4.28 \mathrm{~g}, 89 \%)$ as an oil.

Anhydrous sulfoxide $\mathbf{2 b}$ (3.36 g, 70\%) was obtained via fractional distillation from calcium hydride $(200 \mathrm{mg})$ under reduced pressure at 8-10 Torr $(10 \mathrm{~cm}$ Vigreux column, head temperature: $\left.65-66{ }^{\circ} \mathrm{C}\right)$ as an oil.

${ }^{1} \mathrm{H} \mathrm{NMR}\left(400 \mathrm{MHz}, \mathrm{CDCl}_{3}, 25^{\circ} \mathrm{C}\right)$ :

${ }^{13} \mathrm{C}$ NMR $\left(100 \mathrm{MHz}, \mathrm{CDCl}_{3}, 25{ }^{\circ} \mathrm{C}\right)$ :

FTIR (thin film) $\mathrm{cm}^{-1}$ :

HRMS (DART-TOF+) $m / z$ : $\delta 2.28\left(\mathrm{~s}, 3 \mathrm{H}, \mathrm{C}_{1} \mathbf{H}_{3}\right), 1.15\left(\mathrm{~s}, 9 \mathrm{H}, 3 \times \mathrm{C}_{3} \mathbf{H}_{3}\right)$.

$\delta 52.5\left(\mathbf{C}_{2}\right), 31.6\left(\mathbf{C}_{1}\right), 22.4\left(3 \times \mathbf{C}_{3}\right)$.

$2962(\mathrm{w}), 2869(\mathrm{w}), 1463(\mathrm{w}), 1364(\mathrm{~m}), 1182(\mathrm{w})$, 1036 (s), 932 (m), 691 (m), 569 (m), 468 (m).

$[\mathrm{M}+\mathrm{H}]^{+}$Calcd for $\mathrm{C}_{5} \mathrm{H}_{13} \mathrm{OS}^{+} 121.0682$

Found 121.0682.

TLC (4\% methanol in dichloromethane), Rf: 0.13 (CAM).

B.p.:

$65-66{ }^{\circ} \mathrm{C}(8-10$ Torr $)$. 


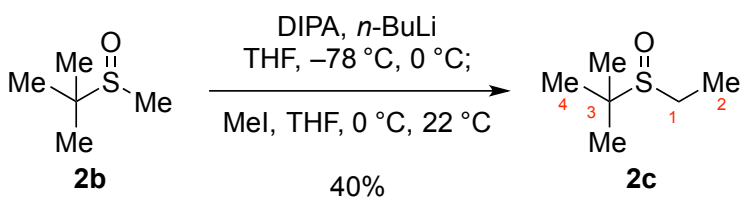

Representative Procedure II for the Alkylation of tert-Butyl Methyl Sulfoxide (2b). tert-Butyl Ethyl Sulfoxide (2c): ${ }^{6}$

A solution of $n$-butyllithium $(2.40 \mathrm{M}$ in hexane, $1.83 \mathrm{~mL}, 4.40 \mathrm{mmol}, 1.10$ equiv) was added via syringe to a colorless solution of $N, N$-diisopropylamine (DIPA, $617 \mu \mathrm{L}, 4.40 \mathrm{mmol}$, 1.10 equiv) in tetrahydrofuran $(20 \mathrm{~mL})$ at $-78^{\circ} \mathrm{C}$. The light yellow solution was stirred at $0{ }^{\circ} \mathrm{C}$ for $15 \mathrm{~min}$, before it was again cooled to $-78^{\circ} \mathrm{C}$. A solution of tert-butyl methyl sulfoxide $(\mathbf{2} \mathbf{b}, 465 \mu \mathrm{L}$, $4.00 \mathrm{mmol}, 1$ equiv) in tetrahydrofuran $(5.0 \mathrm{~mL})$ was added dropwise over $5 \mathrm{~min}$, and afterwards the yellow solution was stirred at $0{ }^{\circ} \mathrm{C}$ for $15 \mathrm{~min}$. Methyl iodide $(261 \mu \mathrm{L}, 4.20 \mathrm{mmol}, 1.05 \mathrm{equiv})$ was added over $2 \mathrm{~min}$, and the light yellow solution was stirred at $22^{\circ} \mathrm{C}$ for $1 \mathrm{~h}$. The reaction mixture was poured into a saturated aqueous solution of ammonium chloride $(20 \mathrm{~mL})$, the phases were separated, and the aqueous layer was extracted with dichloromethane $(10 \times 5 \mathrm{~mL})$. The combined organic extracts were dried over sodium sulfate, were filtered, and were concentrated under reduced pressure. The light yellow crude material was purified via flash column chromatography on silica gel (eluent: $75 \%$ EtOAc in hexanes) to yield sulfoxide $2 \mathrm{c}$ (215 $\mathrm{mg}, 40 \%$ ) as an oil.

${ }^{1} \mathrm{H} \mathrm{NMR}\left(400 \mathrm{MHz}, \mathrm{CDCl}_{3}, 25^{\circ} \mathrm{C}\right)$ :

${ }^{13} \mathrm{C}$ NMR $\left(100 \mathrm{MHz}, \mathrm{CDCl}_{3}, 25{ }^{\circ} \mathrm{C}\right)$ :

FTIR (thin film) $\mathrm{cm}^{-1}$ :

HRMS (ESI-TOF+) $m / z$ :

TLC (EtOAc), Rf: $\delta$ 2.54-2.38 (m, 2H, $\left.\mathrm{C}_{1} \mathbf{H}_{2}\right), 1.37$ (t, $J=7.5 \mathrm{~Hz}, 3 \mathrm{H}$, $\left.\mathrm{C}_{2} \mathbf{H}_{3}\right), 1.22\left(\mathrm{~s}, 9 \mathrm{H}, 3 \times \mathrm{C}_{4} \mathbf{H}_{3}\right)$.

$\delta 52.8\left(\mathbf{C}_{3}\right), 39.1\left(\mathbf{C}_{\mathbf{1}}\right), 22.9\left(3 \times \mathbf{C}_{4}\right), 8.5\left(\mathbf{C}_{\mathbf{2}}\right)$.

$2965(\mathrm{w}), 1461(\mathrm{~m}), 1365(\mathrm{~m}), 1042(\mathrm{~s}), 1009(\mathrm{~s}), 655$ (w), $585(\mathrm{w}), 471(\mathrm{w})$.

$[\mathrm{M}+\mathrm{H}]^{+}$Calcd for $\mathrm{C}_{6} \mathrm{H}_{15} \mathrm{OS}^{+} 135.0838$;

Found 135.0839.

$0.19(\mathrm{CAM})$. 


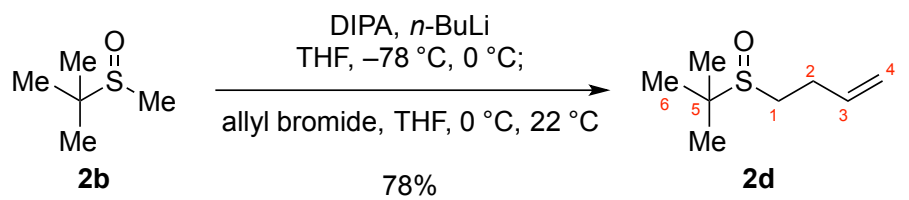

tert-Butyl 3-Butenyl Sulfoxide (2d): 6

Sulfoxide 2d was prepared via Procedure II using tert-butyl methyl sulfoxide $(\mathbf{2 b}, 465 \mu \mathrm{L}$, $4.00 \mathrm{mmol}, 1$ equiv) and allyl bromide ( $363 \mu \mathrm{L}, 4.20 \mathrm{mmol}, 1.05$ equiv). The light yellow solution was stirred at $22^{\circ} \mathrm{C}$ for $15 \mathrm{~h}$, and the crude material was purified via flash column chromatography on silica gel (eluent: $50 \%$ EtOAc in hexanes) to yield sulfoxide $2 \mathrm{~d}(501 \mathrm{mg}, 78 \%$ ) as an oil.

${ }^{1} \mathrm{H}$ NMR $\left(400 \mathrm{MHz}, \mathrm{CDCl}_{3}, 25{ }^{\circ} \mathrm{C}\right): \quad \delta 5.89-5.79\left(\mathrm{~m}, 1 \mathrm{H}, \mathrm{C}_{3} \mathbf{H}\right), 5.13(\mathrm{~d}, J=17.1 \mathrm{~Hz}, 1 \mathrm{H}$, $\left.\mathrm{C}_{4} \mathbf{H}_{\mathbf{a}}\right), 5.06\left(\mathrm{~d}, J=10.1 \mathrm{~Hz}, 1 \mathrm{H}, \mathrm{C}_{4} \mathbf{H}_{\mathbf{b}}\right), 2.64-2.44$ $\left(\mathrm{m}, 4 \mathrm{H}, \mathrm{C}_{1} \mathbf{H}_{2}, \mathrm{C}_{2} \mathbf{H}_{2}\right), 1.22\left(\mathrm{~s}, 9 \mathrm{H}, 3 \times \mathrm{C}_{6} \mathbf{H}_{3}\right)$.

${ }^{13} \mathrm{C} \mathrm{NMR}\left(100 \mathrm{MHz}, \mathrm{CDCl}_{3}, 25^{\circ} \mathrm{C}\right)$ :

$\delta 135.4\left(\mathbf{C}_{\mathbf{3}}\right), 116.9\left(\mathbf{C}_{4}\right), 52.8\left(\mathbf{C}_{\mathbf{5}}\right), 44.9\left(\mathbf{C}_{\mathbf{1}}\right), 27.8$ $\left(\mathbf{C}_{2}\right), 22.8\left(3 \times \mathbf{C}_{6}\right)$.

FTIR (thin film) $\mathrm{cm}^{-1}$ :

$2974(\mathrm{w}), 1641(\mathrm{w}), 1365(\mathrm{~m}), 1183(\mathrm{w}), 1031(\mathrm{~s})$, $914(\mathrm{~m}), 584(\mathrm{w}), 471(\mathrm{~m})$.

HRMS (ESI-TOF+) $m / z$ :

$[\mathrm{M}+\mathrm{H}]^{+}$Calcd for $\mathrm{C}_{8} \mathrm{H}_{17} \mathrm{OS}^{+}$161.0995; Found 161.0994.

TLC (EtOAc), Rf:

0.36 (CAM). 


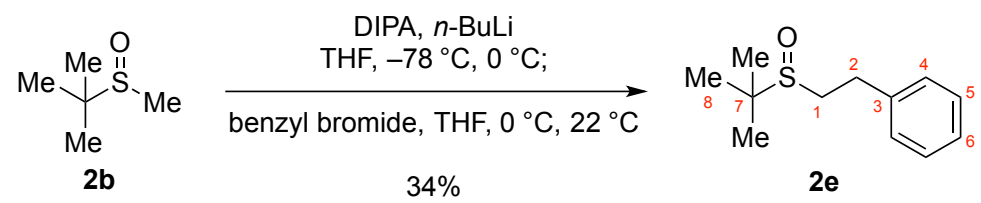

tert-Butyl 2-Phenethyl Sulfoxide (2e):

Sulfoxide 2e was prepared via Procedure II using tert-butyl methyl sulfoxide $(\mathbf{2 b}, 465 \mu \mathrm{L}$, $4.00 \mathrm{mmol}, 1$ equiv) and benzyl bromide $(500 \mu \mathrm{L}, 4.20 \mathrm{mmol}, 1.05$ equiv). The light yellow solution was stirred at $22{ }^{\circ} \mathrm{C}$ for $15 \mathrm{~h}$, and the crude material was purified via flash column chromatography on silica gel (eluent: 50\% EtOAc in hexanes) to yield sulfoxide $2 \mathrm{e}$ ( $283 \mathrm{mg}, 34 \%$ ) as an oil.

${ }^{1} \mathrm{H}$ NMR (400 $\left.\mathrm{MHz}, \mathrm{CDCl}_{3}, 25^{\circ} \mathrm{C}\right)$ :

${ }^{13} \mathrm{C} \mathrm{NMR}\left(100 \mathrm{MHz}, \mathrm{CDCl}_{3}, 25{ }^{\circ} \mathrm{C}\right)$ :

FTIR (thin film) $\mathrm{cm}^{-1}$ :

HRMS (DART-TOF+) $m / z$ :

TLC (EtOAc), Rf: $\delta 7.31\left(\mathrm{t}, J=7.2 \mathrm{~Hz}, 2 \mathrm{H}, 2 \times \mathrm{C}_{5} \mathbf{H}\right), 7.26-7.21(\mathrm{~m}, 3 \mathrm{H}$, $\left.2 \times \mathrm{C}_{4} \mathbf{H}, \mathrm{C}_{6} \mathbf{H}\right), 3.24-3.16\left(\mathrm{~m}, 1 \mathrm{H}, \mathrm{C}_{2} \mathbf{H}_{\mathbf{a}}\right), 3.07-2.99$ $\left(\mathrm{m}, 1 \mathrm{H}, \mathrm{C}_{2} \mathbf{H}_{\mathrm{b}}\right), 2.76-2.65\left(\mathrm{~m}, 2 \mathrm{H}, \mathrm{C}_{1} \mathbf{H}_{2}\right), 1.23(\mathrm{~s}, 9 \mathrm{H}$, $\left.3 \times \mathrm{C}_{8} \mathbf{H}_{3}\right)$.

$\delta 139.4\left(\mathbf{C}_{3}\right), 128.7\left(2 \times \mathbf{C}_{\text {Ar }}\right), 128.6\left(2 \times \mathbf{C}_{\text {Ar }}\right), 126.7$ $\left(\mathbf{C}_{6}\right), 53.0\left(\mathbf{C}_{7}\right), 47.3\left(\mathbf{C}_{1}\right), 30.0\left(\mathbf{C}_{2}\right), 22.9\left(3 \times \mathbf{C}_{8}\right)$.

2962 (w), $1604(\mathrm{w}), 1455(\mathrm{~m}), 1364(\mathrm{~m}), 1176(\mathrm{w})$, 1032 (s), 750 (m), 700 (s), 468 (m).

$[\mathrm{M}+\mathrm{H}]^{+}$Calcd for $\mathrm{C}_{12} \mathrm{H}_{19} \mathrm{OS}^{+} 211.1151$;

Found 211.1146.

0.41 (UV, CAM). 


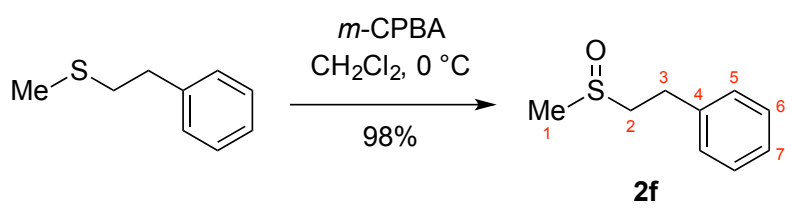

Methyl 2-Phenethyl Sulfoxide (2f):

Sulfoxide 2f was prepared via Procedure I using methyl 2-phenethyl sulfide $(1.51 \mathrm{~g}$, $9.90 \mathrm{mmol}, 1.1$ equiv) and $m$-chloroperbenzoic acid ( $77 \mathrm{wt} \%, 2.0 \mathrm{~g}, 9.0 \mathrm{mmol}, 1$ equiv). The light yellow crude material was purified via flash column chromatography on silica gel (eluent: dichloromethane $\rightarrow 2 \%$ methanol in dichloromethane) to yield sulfoxide $\mathbf{2 f}(1.48 \mathrm{~g}, 98 \%)$ as a solid.

${ }^{1} \mathrm{H}$ NMR $\left(400 \mathrm{MHz}, \mathrm{CDCl}_{3}, 25^{\circ} \mathrm{C}\right): \quad \delta 7.33\left(\mathrm{t}, J=7.4 \mathrm{~Hz}, 2 \mathrm{H}, 2 \times \mathrm{C}_{6} \mathbf{H}\right), 7.29-7.23(\mathrm{~m}, 3 \mathrm{H}$, $\left.2 \times \mathrm{C}_{5} \mathbf{H}, \mathrm{C}_{7} \mathbf{H}\right), 3.17-3.04\left(\mathrm{~m}, 2 \mathrm{H}, \mathrm{C}_{2} \mathbf{H}_{2}\right), 3.03-2.90$ $\left(\mathrm{m}, 2 \mathrm{H}, \mathrm{C}_{3} \mathbf{H}_{2}\right), 2.58\left(\mathrm{~s}, 3 \mathrm{H}, \mathrm{C}_{1} \mathbf{H}_{3}\right)$.

${ }^{13} \mathrm{C} \mathrm{NMR}\left(100 \mathrm{MHz}, \mathrm{CDCl}_{3}, 25{ }^{\circ} \mathrm{C}\right): \quad \delta 138.8\left(\mathbf{C}_{4}\right), 128.8\left(2 \times \mathbf{C}_{6}\right), 128.6\left(2 \times \mathbf{C}_{5}\right), 126.8$ $\left(\mathbf{C}_{7}\right), 56.1\left(\mathbf{C}_{3}\right), 38.7\left(\mathbf{C}_{1}\right), 28.8\left(\mathbf{C}_{2}\right)$.

FTIR (thin film) $\mathrm{cm}^{-1}$ :

$3061(\mathrm{w}), 2913(\mathrm{w}), 1603(\mathrm{w}), 1497(\mathrm{w}), 1454(\mathrm{w})$, $1028(\mathrm{~s}), 974(\mathrm{~m}), 938(\mathrm{~m}), 752(\mathrm{~m}), 699(\mathrm{~s}), 564(\mathrm{w})$, 497 (m).

HRMS (DART-TOF+) $m / z$ :

$[\mathrm{M}+\mathrm{H}]^{+}$Calcd for $\mathrm{C}_{9} \mathrm{H}_{13} \mathrm{OS}^{+}$169.0682;

Found 169.0679 .

TLC (4\% methanol in dichloromethane), Rf: 0.31 (UV, CAM).

M.p.:

$30-32{ }^{\circ} \mathrm{C}\left(\mathrm{CH}_{2} \mathrm{Cl}_{2}\right)$. 

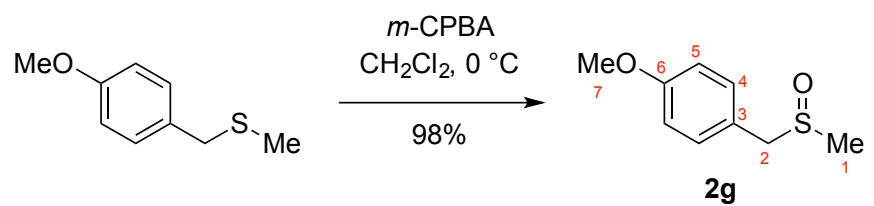

\section{4-Methoxyphenmethyl Methyl Sulfoxide (2g):}

Sulfoxide $\mathbf{2 g}$ was prepared via Procedure I using 4-methoxyphenmethyl methyl sulfide (1.48 g, $8.80 \mathrm{mmol}, 1.1$ equiv) and $m$-chloroperbenzoic acid (77 wt $\%, 1.8 \mathrm{~g}, 8.0 \mathrm{mmol}, 1$ equiv). The light yellow crude material was purified via flash column chromatography on silica gel (eluent: dichloromethane $\rightarrow 2 \%$ methanol in dichloromethane) to yield sulfoxide $2 \mathrm{~g}(1.45 \mathrm{~g}, 98 \%)$ as a solid.

${ }^{1} \mathrm{H} \mathrm{NMR}\left(400 \mathrm{MHz}, \mathrm{CDCl}_{3}, 25^{\circ} \mathrm{C}\right)$ :

${ }^{13} \mathrm{C}$ NMR $\left(100 \mathrm{MHz}, \mathrm{CDCl}_{3}, 25^{\circ} \mathrm{C}\right):$

FTIR (thin film) $\mathrm{cm}^{-1}$ :

HRMS (DART-TOF+) $m / z$ : $\delta 7.19\left(\mathrm{~d}, J=8.3 \mathrm{~Hz}, 2 \mathrm{H}, 2 \times \mathrm{C}_{4} \mathbf{H}\right), 6.89(\mathrm{~d}, J=$ $\left.8.5 \mathrm{~Hz}, 2 \mathrm{H}, 2 \times \mathrm{C}_{5} \mathbf{H}\right), 3.97(\mathrm{~d}, J=13.0 \mathrm{~Hz}, 1 \mathrm{H}$, $\left.\mathrm{C}_{2} \mathbf{H}_{\mathbf{a}}\right), 3.86\left(\mathrm{~d}, J=13.0 \mathrm{~Hz}, 1 \mathrm{H}, \mathrm{C}_{2} \mathbf{H}_{\mathbf{b}}\right), 3.79(\mathrm{~s}, 3 \mathrm{H}$, $\left.\mathrm{C}_{7} \mathbf{H}_{3}\right), 2.41$ (s, 3H, $\left.\mathrm{C}_{1} \mathbf{H}_{3}\right)$.

$\delta 159.8\left(\mathbf{C}_{6}\right), 131.3\left(2 \times \mathbf{C}_{4}\right), 121.6\left(\mathbf{C}_{3}\right), 114.5(2 \times$ $\left.\mathbf{C}_{5}\right), 59.7\left(\mathbf{C}_{2}\right), 55.4\left(\mathbf{C}_{7}\right), 37.2\left(\mathbf{C}_{1}\right)$.

$2955(\mathrm{w}), 2907(\mathrm{w}), 2836(\mathrm{w}), 1694(\mathrm{~m}), 1512(\mathrm{~m})$, $1243(\mathrm{~m}), 1173(\mathrm{~m}), 1106(\mathrm{~m}), 1026(\mathrm{~s}), 835(\mathrm{~m}), 681$ (w), $553(\mathrm{~m}), 513(\mathrm{~s})$.

$[\mathrm{M}+\mathrm{H}]^{+}$Calcd for $\mathrm{C}_{9} \mathrm{H}_{13} \mathrm{O}_{2} \mathrm{~S}^{+}$185.0631;

Found 185.0629 .

TLC (4\% methanol in dichloromethane), Rf: 0.29 (UV, CAM).

M.p.:

$51-53{ }^{\circ} \mathrm{C}\left(\mathrm{CH}_{2} \mathrm{Cl}_{2}\right)$. 


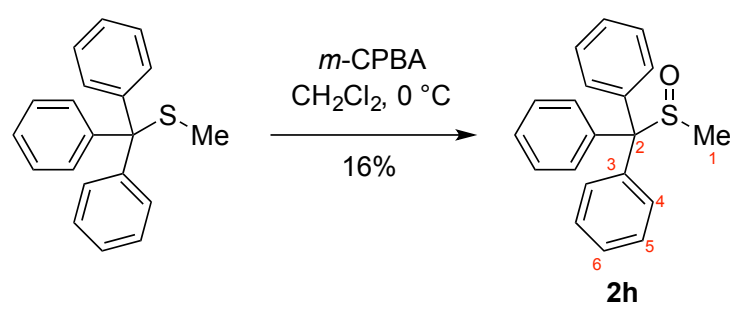

\section{Triphenylmethyl Methyl Sulfoxide (2h): $:^{5}$}

Sulfoxide $\mathbf{2 h}$ was prepared via Procedure I using triphenmethyl methyl sulfide (1.28 g, $4.40 \mathrm{mmol}, 1.1$ equiv) and $m$-chloroperbenzoic acid $(77 \mathrm{wt} \%, 900 \mathrm{mg}, 4.0 \mathrm{mmol}, 1$ equiv). After stirring the colorless suspension at $0^{\circ} \mathrm{C}$ for $1 \mathrm{~h}$, an aqueous solution of sodium hydroxide $(1 \mathrm{M}$, $15 \mathrm{~mL}$ ) was added. The light yellow crude material was purified via flash column chromatography on silica gel (eluent: 10\% EtOAc in hexanes) to yield sulfoxide $\mathbf{2 h}(200 \mathrm{mg}, 16 \%)$ as a solid.

${ }^{1} \mathrm{H} \mathrm{NMR}\left(400 \mathrm{MHz}, \mathrm{CD}_{2} \mathrm{Cl}_{2}, 25^{\circ} \mathrm{C}\right)$ :

${ }^{13} \mathrm{C}$ NMR $\left(100 \mathrm{MHz}, \mathrm{CD}_{2} \mathrm{Cl}_{2}, 25^{\circ} \mathrm{C}\right)$ :

FTIR (thin film) $\mathrm{cm}^{-1}$ :

HRMS (ESI-TOF+) $m / z$ :

TLC (25\% EtOAc in hexanes), Rf:

M.p.: $\delta$ 7.43-7.35 (m, 15H, $\left.6 \times \mathrm{C}_{4} \mathbf{H}, 6 \times \mathrm{C}_{5} \mathbf{H}, 3 \times \mathrm{C}_{6} \mathbf{H}\right)$, $2.82\left(\mathrm{~s}, 3 \mathrm{H}, \mathrm{C}_{1} \mathbf{H}_{3}\right)$.

$\delta 139.4\left(3 \times \mathbf{C}_{3}\right), 131.4\left(6 \times \mathbf{C}_{A r}\right), 129.0\left(3 \times \mathbf{C}_{6}\right)$, $128.8\left(6 \times \mathbf{C}_{\mathrm{Ar}}\right), 86.8\left(\mathbf{C}_{2}\right), 42.8\left(\mathbf{C}_{\mathbf{1}}\right)$.

$2933(\mathrm{w}), 1490(\mathrm{w}), 1440(\mathrm{w}), 1127(\mathrm{~s}), 953$ (w), 726 (m), $697(\mathrm{~s}), 538(\mathrm{~s}), 482(\mathrm{~m}), 471(\mathrm{~m})$.

$[\mathrm{M}+\mathrm{H}]^{+}$Calcd for $\mathrm{C}_{20} \mathrm{H}_{19} \mathrm{OS}^{+} 307.1151$;

Found 307.1139.

0.45 (UV, CAM).

$178-180{ }^{\circ} \mathrm{C}\left(\mathrm{CH}_{2} \mathrm{Cl}_{2}\right.$, decomposition $)$. 
<smiles>O=C(Cc1ccccc1)N1CCCC1</smiles>

$1 b$

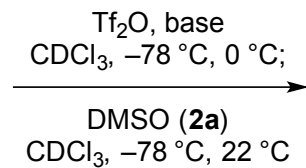

$\mathrm{CDCl}_{3},-78^{\circ} \mathrm{C}, 22^{\circ} \mathrm{C}$

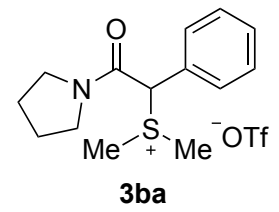

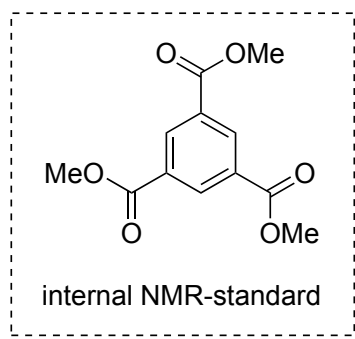

Representative Procedure for Entry 3 of Table S1: Screening of Reaction Conditions for the Formation of Sulfonium Trifluoromethanesulfonate 3ba:

Trifluoromethanesulfonic anhydride $(46.3 \mu \mathrm{L}, 275 \mu \mathrm{mol}, 1.10$ equiv) was added via syringe over $1 \mathrm{~min}$ to a stirred solution of pyrrolidine amide $1 \mathbf{b}(47.3 \mathrm{mg}, 250 \mu \mathrm{mol}, 1$ equiv), trimethyl-1,3,5-benzene tricarboxylate $(21.0 \mathrm{mg}, 83.3 \mu \mathrm{mol}, 0.33$ equiv) and 2-chloropyridine $\left(47.3 \mu \mathrm{L}, 500 \mu \mathrm{mol}, 2.00\right.$ equiv) in chloroform- $d(800 \mu \mathrm{L})$ at $-78^{\circ} \mathrm{C}$ under argon atmosphere. The dark red solution was stirred at $0{ }^{\circ} \mathrm{C}$ for $15 \mathrm{~min}$, before it was again cooled to $-78{ }^{\circ} \mathrm{C}$. A solution of dimethyl sulfoxide (2a, $26.6 \mu \mathrm{L}, 375 \mu \mathrm{mol}, 1.50$ equiv) in chloroform- $d$ (200 $\mu \mathrm{L})$ was added via cannula over $2 \mathrm{~min}$ at $-78{ }^{\circ} \mathrm{C}$. The reaction mixture was stirred at $0{ }^{\circ} \mathrm{C}$ for $15 \mathrm{~min}$, then at $22{ }^{\circ} \mathrm{C}$ for $30 \mathrm{~min}$ to give a yellow solution. This solution $(300 \mu \mathrm{L})$ was transferred into an NMR-tube under intert atmosphere, was diluted with chloroform- $d(350 \mu \mathrm{L})$, and was measured on a Bruker AVANCE III 400 spectrometer with a relaxation delay of $25 \mathrm{~s}$. The NMR-yield was determined with respect to the internal standard.

Table S1: Screening of reaction conditions for the formation of sulfonium trifluoromethanesulfonate $\mathbf{3 b a}$ with dimethyl sulfoxide (2a).

\begin{tabular}{|c|c|c|c|c|c|c|c|}
\hline Entry & \multicolumn{2}{|c|}{ Base } & $\mathbf{T f}_{2} \mathrm{O}$ & DMSO (2a) & Temp. ${ }^{[a]}$ & Time $^{[\mathrm{a}]}$ & NMR-yield \\
\hline 1 & - & - & 1.10 equiv & 1.50 equiv & $-78^{\circ} \mathrm{C}, 0^{\circ} \mathrm{C}$ & $15 \mathrm{~min}$ & n.r. \\
\hline 2 & 2-FPy & 2.00 equiv & 1.10 equiv & 1.50 equiv & $-78^{\circ} \mathrm{C}, 0^{\circ} \mathrm{C}$ & $15 \mathrm{~min}$ & $36 \%$ \\
\hline 3 & 2-ClPy & 2.00 equiv & 1.10 equiv & 1.50 equiv & $-78^{\circ} \mathrm{C}, 0^{\circ} \mathrm{C}$ & $15 \mathrm{~min}$ & $63 \%\left(63 \%{ }^{[\mathrm{b}]}\right)$ \\
\hline 4 & 2-BrPy & 2.00 equiv & 1.10 equiv & 1.50 equiv & $-78^{\circ} \mathrm{C}, 0^{\circ} \mathrm{C}$ & $15 \mathrm{~min}$ & $63 \%$ \\
\hline 5 & 2-IPy & 2.00 equiv & 1.10 equiv & 1.50 equiv & $-78^{\circ} \mathrm{C}, 0^{\circ} \mathrm{C}$ & $15 \mathrm{~min}$ & $\sim 72 \%\left(60 \% 0^{[b]}\right)$ \\
\hline 6 & 2-MeOPy & 2.00 equiv & 1.10 equiv & 1.50 equiv & $-78^{\circ} \mathrm{C}, 0^{\circ} \mathrm{C}$ & $15 \mathrm{~min}$ & $\sim 87 \%\left(60 \%{ }^{[b]}\right)$ \\
\hline 7 & 2-MePy & 2.00 equiv & 1.10 equiv & 1.50 equiv & $-78^{\circ} \mathrm{C}, 0^{\circ} \mathrm{C}$ & $15 \mathrm{~min}$ & $<15 \%$ \\
\hline 8 & 2,6-DMeOPy & 2.00 equiv & 1.10 equiv & 1.50 equiv & $-78^{\circ} \mathrm{C}, 0^{\circ} \mathrm{C}$ & $15 \mathrm{~min}$ & $63 \%$ \\
\hline 9 & 2,4-DMeOPy & 2.00 equiv & 1.10 equiv & 1.50 equiv & $-78^{\circ} \mathrm{C}, 0^{\circ} \mathrm{C}$ & $15 \mathrm{~min}$ & $<15 \%$ \\
\hline 10 & 2-ClPy & 2.00 equiv & 1.10 equiv & 1.50 equiv & $0^{\circ} \mathrm{C}$ & $15 \mathrm{~min}$ & $59 \%$ \\
\hline 11 & 2-ClPy & 2.00 equiv & 1.10 equiv & 1.50 equiv & $-78^{\circ} \mathrm{C}$ & $15 \mathrm{~min}$ & n.r. \\
\hline 12 & 2-ClPy & 2.00 equiv & 1.10 equiv & 1.50 equiv & $-78^{\circ} \mathrm{C}, 0{ }^{\circ} \mathrm{C}$ & $5 \mathrm{~min}$ & $75 \%\left(63 \% 0^{[b]}\right)$ \\
\hline 13 & 2-ClPy & 2.00 equiv & 1.10 equiv & 1.50 equiv & $-78^{\circ} \mathrm{C}, 0^{\circ} \mathrm{C}$ & $30 \mathrm{~min}$ & $59 \%$ \\
\hline 14 & 2-CIPy & 1.20 equiv & 1.10 equiv & 1.50 equiv & $-78^{\circ} \mathrm{C}, 0^{\circ} \mathrm{C}$ & $15 \mathrm{~min}$ & $20 \%$ \\
\hline 15 & 2-CIPy & 3.00 equiv & 1.10 equiv & 1.50 equiv & $-78^{\circ} \mathrm{C}, 0{ }^{\circ} \mathrm{C}$ & $15 \mathrm{~min}$ & $74 \%\left(71 \% 0^{[\mathrm{b}]}\right)$ \\
\hline 16 & 2-ClPy & 5.00 equiv & 1.10 equiv & 1.50 equiv & $-78^{\circ} \mathrm{C}, 0^{\circ} \mathrm{C}$ & $15 \mathrm{~min}$ & $66 \%$ \\
\hline 17 & 2-ClPy & 2.00 equiv & 1.10 equiv & 1.10 equiv & $-78^{\circ} \mathrm{C}, 0^{\circ} \mathrm{C}$ & $15 \mathrm{~min}$ & $62 \%$ \\
\hline 18 & 2-ClPy & 2.00 equiv & 1.10 equiv & 3.00 equiv & $-78^{\circ} \mathrm{C}, 0{ }^{\circ} \mathrm{C}$ & $15 \mathrm{~min}$ & $64 \%$ \\
\hline 19 & 2-CIPy & 2.00 equiv & 1.00 equiv & 1.50 equiv & $-78^{\circ} \mathrm{C}, 0^{\circ} \mathrm{C}$ & $15 \mathrm{~min}$ & $67 \%$ \\
\hline 20 & 2-CIPy & 2.00 equiv & 1.30 equiv & 1.50 equiv & $-78^{\circ} \mathrm{C}, 0^{\circ} \mathrm{C}$ & $15 \mathrm{~min}$ & $51 \%$ \\
\hline $21^{[\mathrm{c}]}$ & 2-CIPy & 2.00 equiv & 1.10 equiv & 1.50 equiv & $-78^{\circ} \mathrm{C}, 0^{\circ} \mathrm{C}$ & $15 \mathrm{~min}$ & $73 \%$ \\
\hline $22^{[\mathrm{d}]}$ & 2-ClPy & 2.00 equiv & 1.10 equiv & 1.50 equiv & $-78^{\circ} \mathrm{C}, 0^{\circ} \mathrm{C}$ & $15 \mathrm{~min}$ & $61 \%$ \\
\hline $23^{[\mathrm{c}]}$ & 2-CIPy & 3.00 equiv & 1.05 equiv & 1.20 equiv & $-78^{\circ} \mathrm{C}, 0^{\circ} \mathrm{C}$ & $15 \mathrm{~min}$ & 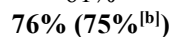 \\
\hline
\end{tabular}

n.r.: No reaction.

${ }^{[a]}$ : Temperatures and reaction times after the addition of substituted pyrine base.

[b]: Isolated yield determined after dealkylation with 10.0 equiv $\mathrm{Et}_{3} \mathrm{~N}$ in $\mathrm{MeCN}\left(60{ }^{\circ} \mathrm{C}, 15 \mathrm{~h}\right)$.

${ }^{[c]}$ : Pyrrolidine amide $\mathbf{1 b}$ was azeotropically dried with anhydrous benzene $(1.0 \mathrm{~mL})$ three times.

[d]: Activated $3 \AA$ molecular sieves $(10 \mathrm{mg})$ were added to the reaction solution.

$\mathrm{Tf}_{2} \mathrm{O}$ : Trifluoromethanesulfonic anhydride; DMSO: Dimethyl sulfoxide; 2-FPy: 2-Fluoropyridine; 2-ClPy: 2Chloropyridine; 2-BrPy: 2-Bromopyridine; 2-IPy: 2-Iodopyridine; 2-MeOPy: 2-Methoxypyridine; 2-MePy: 2Methylpyridine; 2,6-DMeOPy: 2,6-Dimethoxypyridine; 2,4-DMeOPy: 2,4-Dimethoxypyridine. 

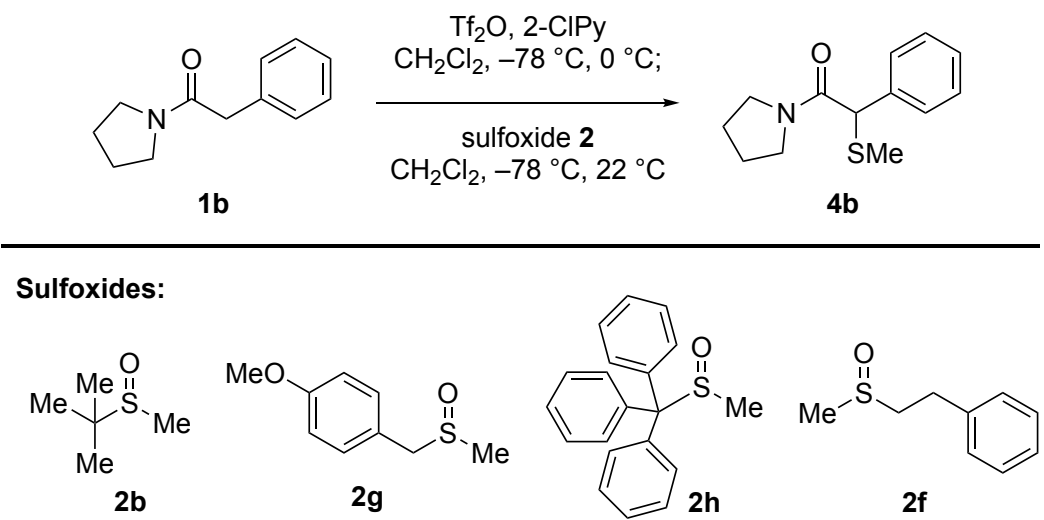

\section{Representative Procedure for Entry 1 of Table S2: Screening of Sulfoxides for the} Spontaneous Sulfide Formation:

Trifluoromethanesulfonic anhydride $(92.5 \mu \mathrm{L}, 550 \mu \mathrm{mol}, 1.10$ equiv) was added via syringe over $1 \mathrm{~min}$ to a stirred solution of pyrrolidine amide $\mathbf{1 b}(94.6 \mathrm{mg}, 500 \mu \mathrm{mol}, 1 \mathrm{equiv})$ and 2-chloropyridine $\left(94.6 \mu \mathrm{L}, 1.00 \mu \mathrm{mol}, 2.00\right.$ equiv) in dichloromethane $(1.7 \mathrm{~mL})$ at $-78^{\circ} \mathrm{C}$ under argon atmosphere. The dark red solution was stirred at $0{ }^{\circ} \mathrm{C}$ for $15 \mathrm{~min}$, before it was again cooled to $-78{ }^{\circ} \mathrm{C}$. A solution of tert-butyl methyl sulfoxide $(\mathbf{2 b}, 87.3 \mu \mathrm{L}, 750 \mu \mathrm{mol}, 1.50$ equiv) in dichloromethane $(200 \mu \mathrm{L})$ was added via cannula over $2 \mathrm{~min}$ at $-78^{\circ} \mathrm{C}$. The reaction mixture was stirred at $0{ }^{\circ} \mathrm{C}$ for $15 \mathrm{~min}$, then at $22{ }^{\circ} \mathrm{C}$ for $30 \mathrm{~min}$ to give a yellow solution. A saturated aqueous solution of sodium bicarbonate $(2.0 \mathrm{~mL})$ and dichloromethane $(1.0 \mathrm{~mL})$ were added subsequently, and the yellow biphasic mixture was vigorously stirred at $22{ }^{\circ} \mathrm{C}$ for $15 \mathrm{~min}$. After separation of the organic layer, the aqueous phase was extracted with dichloromethane $(3 \times 1.0 \mathrm{~mL})$. The combined light yellow organic extracts were dried over sodium sulfate, were filtered, and were concentrated under reduced pressure. The light yellow oily crude material was purified via flash column chromatography on silica gel (eluent: $25 \%$ EtOAc in hexanes) to yield $\alpha$-SMe-pyrrolidine amide $4 \mathbf{b}(68.3 \mathrm{mg}, 58 \%)$ as a solid.

Table S2: Screening of sulfoxides for the spontaneous sulfide formation.

\begin{tabular}{|c|c|c|c|}
\hline Entry & Sulfoxide & Time $^{[a]}$ & Isolated yield \\
\hline 1 & $2 \mathbf{b}$ & $0^{\circ} \mathrm{C}, 15 \min , 22{ }^{\circ} \mathrm{C}, 30 \mathrm{~min}$ & $\mathbf{5 8 \%}$ \\
\hline 2 & $2 \mathrm{~g}$ & $0^{\circ} \mathrm{C}, 15 \min , 22^{\circ} \mathrm{C}, 30 \min$ & $56 \%$ \\
\hline 3 & $2 \mathrm{~h}$ & $0^{\circ} \mathrm{C}, 15 \mathrm{~min}, 22^{\circ} \mathrm{C}, 30 \mathrm{~min}$ & $<5 \%$ \\
\hline 4 & $2 f$ & $0^{\circ} \mathrm{C}, 15 \mathrm{~min}, 22^{\circ} \mathrm{C}, 30 \mathrm{~min}$ & n.s.d. $\left(41 \%\left[{ }^{[b]}\right)\right.$ \\
\hline 5 & $2 \mathbf{b}$ & $0{ }^{\circ} \mathrm{C}, 1 \mathrm{~min}$ & $58 \%$ \\
\hline 6 & $2 \mathbf{b}$ & $15 \min$ & $56 \%$ \\
\hline 7 & $2 \mathbf{b}$ & $0^{\circ} \mathrm{C}, 15 \mathrm{~min}, 22^{\circ} \mathrm{C}, 3 \mathrm{~h}$ & $56 \%$ \\
\hline \multicolumn{4}{|c|}{$\begin{array}{l}\text { n.s.d.: No spontaneous dealkylation. } \\
\text { [a]: Temperatures and reaction times after the addition of the correspondin } \\
\text { sulfoxide. } \\
{[\text { [b]: Corresponding }(S)-(2-\text { phenethyl)amide isolated after dealkylation wit }} \\
\text { Et } 3 \mathrm{~N}\left(10.0 \text { equiv) in } \mathrm{MeCN}\left(60^{\circ} \mathrm{C}, 15 \mathrm{~h}\right) \text {. }\right.\end{array}$} \\
\hline
\end{tabular}


<smiles>O=C(CCc1ccccc1)N1CCCC1</smiles>

$1 t$

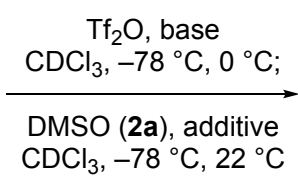

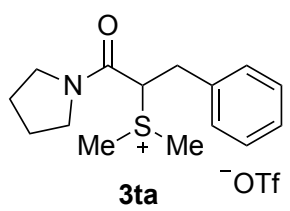

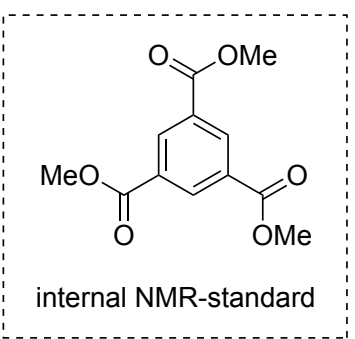

Representative Procedure for Entry 4 of Table S3: Screening of Reaction Conditions for the Formatiom of Sulfonium Trifluoromethanesulfonate 3ta:

Trifluoromethanesulfonic anhydride $(46.3 \mu \mathrm{L}, 275 \mu \mathrm{mol}, 1.10$ equiv) was added via syringe over $1 \mathrm{~min}$ to a stirred solution of pyrrolidine amide $\mathbf{1 t}(50.8 \mathrm{mg}, 250 \mu \mathrm{mol}, 1$ equiv), trimethyl-1,3,5-benzene tricarboxylate $(21.0 \mathrm{mg}, 83.3 \mu \mathrm{mol}, 0.33$ equiv) and 2-chloropyridine $\left(71.0 \mu \mathrm{L}, 750 \mu \mathrm{mol}, 3.00\right.$ equiv) in chloroform- $d(800 \mu \mathrm{L})$ at $-78^{\circ} \mathrm{C}$ under argon atmosphere. The red solution was stirred at $0{ }^{\circ} \mathrm{C}$ for $15 \mathrm{~min}$, before it was again cooled to $-78{ }^{\circ} \mathrm{C}$. A solution of dimethyl sulfoxide (2a, $44.4 \mu \mathrm{L}, 625 \mu \mathrm{mol}, 2.50$ equiv) in chloroform- $d$ ( $200 \mu \mathrm{L})$ was added via cannula over $2 \mathrm{~min}$, followed by addition of trifluoromethanesulfonic anhydride $(42.1 \mu \mathrm{L}$, $250 \mu \mathrm{mol}, 1.00$ equiv) via syringe over $1 \mathrm{~min}$ at $-78^{\circ} \mathrm{C}$. The reaction mixture was stirred at $0{ }^{\circ} \mathrm{C}$ for $15 \mathrm{~min}$, then at $22^{\circ} \mathrm{C}$ for $30 \mathrm{~min}$ to give a yellow solution. This solution $(300 \mu \mathrm{L})$ was transferred into an NMR-tube under intert atmosphere, was diluted with chloroform- $d(350 \mu \mathrm{L})$, and was measured on a Bruker AVANCE III 400 spectrometer with a relaxation delay of $25 \mathrm{~s}$. The NMR-yield was determined with respect to the internal standard.

Table S3: Screening of reaction conditions for the formation of sulfonium trifluoromethanesulfonate 3 ta with dimethyl sulfoxide

\begin{tabular}{|c|c|c|c|c|}
\hline \multirow{2}{*}{$\frac{\text { Entry }}{1}$} & \multicolumn{2}{|c|}{ Additive } & \multirow{2}{*}{$\frac{\text { DMSO (2a) }}{2.50 \text { equiv }}$} & \multirow{2}{*}{$\frac{\text { NMR-yield }}{<5 \%}$} \\
\hline & $\mathrm{Tf}_{2} \mathrm{O}$ & - & & \\
\hline 2 & $\mathrm{Tf}_{2} \mathrm{O}$ & 0.20 equiv & 2.50 equiv & $17 \%$ \\
\hline 3 & $\mathrm{Tf}_{2} \mathrm{O}$ & 0.60 equiv & 2.50 equiv & $46 \%$ \\
\hline 4 & $\mathrm{Tf}_{2} \mathrm{O}$ & 1.00 equiv & 2.50 equiv & $79 \%$ \\
\hline 5 & $\mathrm{Tf}_{2} \mathrm{O}$ & 1.40 equiv & 2.50 equiv & $12 \%$ \\
\hline 6 & $\mathrm{Ms}_{2} \mathrm{O}$ & 1.00 equiv & 2.50 equiv & $33 \%$ \\
\hline 7 & TFAA & 0.20 equiv & 2.50 equiv & $40 \%$ \\
\hline 8 & TFAA & 0.60 equiv & 2.50 equiv & $77 \%$ \\
\hline 9 & TFAA & 1.00 equiv & 2.50 equiv & $84 \%$ \\
\hline 10 & TFAA & 1.40 equiv & 2.50 equiv & $81 \%$ \\
\hline 11 & TFAA & 1.00 equiv & 5.00 equiv & $81 \%$ \\
\hline $12^{[\mathrm{a}]^{-}}$ & $\mathrm{MsOH}$ & 1.00 equiv & 1.20 equiv & $33 \%$ \\
\hline $13^{[\mathrm{a}]}$ & TFA & 1.00 equiv & 1.20 equiv & $45 \%$ \\
\hline $14^{[\mathrm{a}]}$ & TCA & 1.00 equiv & 1.20 equiv & $46 \%$ \\
\hline $15^{[\mathrm{a}]}$ & $\mathrm{AcOH}$ & 1.00 equiv & 1.20 equiv & $11 \%$ \\
\hline $16^{[\mathrm{a}]}$ & DBP & 1.00 equiv & 1.20 equiv & $<5 \%$ \\
\hline
\end{tabular}

[a]: $\mathrm{Tf}_{2} \mathrm{O}$ ( 1.05 equiv) was used for amide activation.

DMSO: Dimethyl sulfoxide; $\mathrm{Tf}_{2} \mathrm{O}$ : Trifluoromethanesulfonic anhydride; $\mathrm{Ms}_{2} \mathrm{O}$ : Methanesulfonic anhydride; TFAA: Trifluoroacetic anhydride; $\mathrm{MsOH}$ : Methanesulfonic acid; TFA: Trifluoroacetic acid; TCA: Trichloroacetic acid; AcOH: Acetic acid; DBP: Di- $n$-butyl phosphate. 
<smiles>O=C(Cc1ccccc1)N1CCOCC1</smiles>

$1 \mathrm{a}$

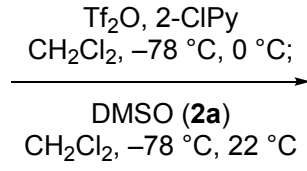

$\mathrm{CH}_{2} \mathrm{Cl}_{2},-78^{\circ} \mathrm{C}, 22^{\circ} \mathrm{C}$

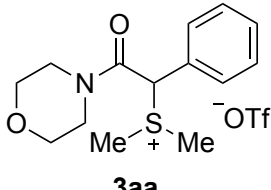

3aa

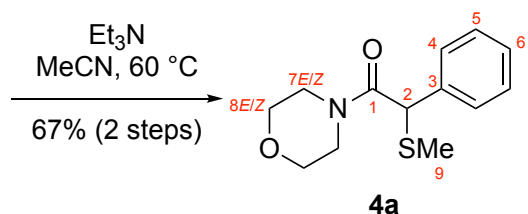

$4 a$

Method A: Representative Procedure for the $\alpha$-Sulfidation of Benzylic Amides with Methyl Sulfoxides. $\alpha$-SMe-Morpholine Amide 4a:

Trifluoromethanesulfonic anhydride $(88.3 \mu \mathrm{L}, 525 \mu \mathrm{mol}, 1.05$ equiv) was added via syringe over $1 \mathrm{~min}$ to a stirred solution of azeotropically dried morpholine amide $1 \mathrm{a}$ (103 $\mathrm{mg}$, $500 \mu \mathrm{mol}, 1$ equiv) and 2-chloropyridine $(142 \mu \mathrm{L}, 1.50 \mathrm{mmol}, 3.00$ equiv) in dichloromethane $(1.7 \mathrm{~mL})$ at $-78^{\circ} \mathrm{C}$ under argon atmosphere. The dark red solution was stirred at $0{ }^{\circ} \mathrm{C}$ for $15 \mathrm{~min}$, before it was again cooled to $-78^{\circ} \mathrm{C}$. A solution of dimethyl sulfoxide $(2 \mathbf{a}, 42.6 \mu \mathrm{L}, 600 \mu \mathrm{mol}$, 1.20 equiv) in dichloromethane $(0.3 \mathrm{~mL})$ was added via cannula over $2 \mathrm{~min}$ at $-78^{\circ} \mathrm{C}$. The reaction mixture was stirred at $0{ }^{\circ} \mathrm{C}$ for $15 \mathrm{~min}$, then at $22{ }^{\circ} \mathrm{C}$ for $30 \mathrm{~min}$ to give a yellow solution, which was concentrated under reduced pressure. The orange oily residue was dissolved in acetonitrile $(2.0 \mathrm{~mL})$. Triethylamine $(697 \mu \mathrm{L}, 5.00 \mathrm{mmol}, 10.0$ equiv) was added, and the resulting brown solution was stirred in a sealed roundbottom flask at $60{ }^{\circ} \mathrm{C}$ for $15 \mathrm{~h}$. After concentration under reduced pressure, the brown oily crude material was purified via flash column chromatography on silica gel (eluent: 25\% EtOAc in hexanes) to yield $\alpha$-SMe-morpholine amide 4a $(84.4 \mathrm{mg}, 67 \%)$ as a solid. Structural assignments were made using additional information from gCOSY, gHSQC and $\mathrm{gHMBC}$ experiments.

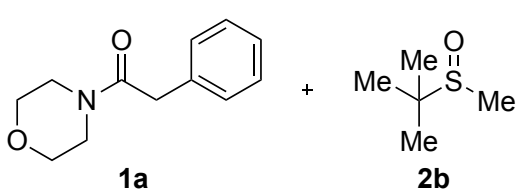

$1 \mathrm{a}$

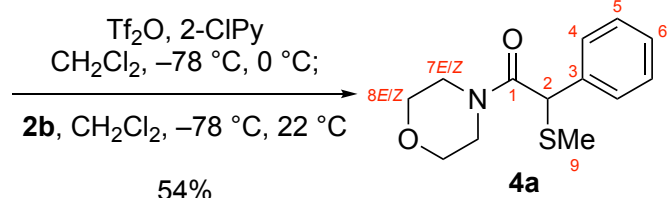

$54 \%$

Method B: Representative Procedure for the $\alpha$-Sulfidation of Benzylic Amides with tertButyl Sulfoxides. $\alpha$-SMe-Morpholine Amide 4a:

Trifluoromethanesulfonic anhydride $(88.3 \mu \mathrm{L}, 525 \mu \mathrm{mol}, 1.05$ equiv) was added via syringe over $1 \mathrm{~min}$ to a stirred solution of azeotropically dried morpholine amide $1 \mathbf{a}$ (103 $\mathrm{mg}$, $500 \mu \mathrm{mol}, 1$ equiv) and 2-chloropyridine $(142 \mu \mathrm{L}, 1.50 \mathrm{mmol}, 3.00$ equiv) in dichloromethane $(1.7 \mathrm{~mL})$ at $-78^{\circ} \mathrm{C}$ under argon atmosphere. The dark red solution was stirred at $0{ }^{\circ} \mathrm{C}$ for $15 \mathrm{~min}$, before it was again cooled to $-78^{\circ} \mathrm{C}$. A solution of tert-butyl methyl sulfoxide $(\mathbf{2} \mathbf{b}, 69.8 \mu \mathrm{L}$, $600 \mu \mathrm{mol}, 1.20$ equiv) in dichloromethane $(0.3 \mathrm{~mL})$ was added via cannula over $2 \mathrm{~min}$ at $-78^{\circ} \mathrm{C}$. The reaction mixture was stirred at $0{ }^{\circ} \mathrm{C}$ for $15 \mathrm{~min}$, then at $22^{\circ} \mathrm{C}$ for $30 \mathrm{~min}$ to give a yellow solution. A saturated aqueous solution of sodium bicarbonate $(2.0 \mathrm{~mL})$ followed by dichloromethane $(1.0 \mathrm{~mL})$ was added, and the yellow biphasic mixture was vigorously stirred at $22{ }^{\circ} \mathrm{C}$ for $15 \mathrm{~min}$. After separation of the organic layer, the aqueous phase was extracted with dichloromethane $(3 \times 1.0 \mathrm{~mL})$. The combined light yellow organic extracts were dried over sodium sulfate, were filtered, and were concentrated under reduced pressure. The light yellow oily crude material was purified via flash column chromatography on silica gel (eluent: $25 \%$ EtOAc in hexanes) to yield $\alpha$-SMe-morpholine amide $4 \mathbf{a}(67.6 \mathrm{mg}, 54 \%)$ as a solid. The analytic data is fully consistent with that of compound $\mathbf{4 a}$ from Method A.

${ }^{1} \mathrm{H}$ NMR $\left(400 \mathrm{MHz}, \mathrm{CDCl}_{3}, 25^{\circ} \mathrm{C}\right): \quad \delta 7.39-7.33\left(\mathrm{~m}, 4 \mathrm{H}, 2 \times \mathrm{C}_{4} \mathbf{H}, 2 \times \mathrm{C}_{5} \mathbf{H}\right), 7.30-7.26$ $\left(\mathrm{m}, 1 \mathrm{H}, \mathrm{C}_{6} \mathbf{H}\right), 4.71\left(\mathrm{~s}, 1 \mathrm{H}, \mathrm{C}_{2} \mathbf{H}\right), 3.72-3.43(\mathrm{~m}, 6 \mathrm{H}$, 
$\left.3 \times \mathrm{C}_{7 E / Z} \mathbf{H}, 3 \times \mathrm{C}_{8 E / Z} \mathbf{H}\right), 3.37-3.32\left(\mathrm{~m}, 1 \mathrm{H}, \mathrm{C}_{7 E / Z} \mathbf{H}\right)$, 3.28-3.23 (m, 1H, $\left.\mathrm{C}_{8 E / Z} \mathbf{H}\right), 2.01$ ( $\left.\mathrm{s}, 3 \mathrm{H}, \mathrm{C}_{9} \mathbf{H}_{3}\right)$.

${ }^{13} \mathrm{C} \mathrm{NMR}\left(100 \mathrm{MHz}, \mathrm{CDCl}_{3}, 25{ }^{\circ} \mathrm{C}\right)$ :

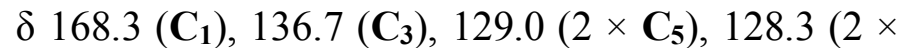
$\left.\mathbf{C}_{4}\right), 128.0\left(\mathbf{C}_{6}\right), 66.9\left(\mathbf{C}_{8 E / Z}\right), 66.4\left(\mathbf{C}_{8 E / Z}\right), 53.5\left(\mathbf{C}_{2}\right)$, $46.6\left(\mathbf{C}_{7 E / Z}\right), 42.8\left(\mathbf{C}_{7 E / Z}\right), 14.7\left(\mathbf{C}_{9}\right)$.

FTIR (thin film) $\mathrm{cm}^{-1}$ :

$2956(\mathrm{w}), 2905$ (w), $2852(\mathrm{w}), 1621$ (s), 1439 (s), 1223 (s), 1112 (s), $1036(\mathrm{~m}), 963(\mathrm{w}), 853(\mathrm{~m}), 733$ (s), $719(\mathrm{~s}), 697(\mathrm{~s}), 647(\mathrm{~m}), 576(\mathrm{~s}), 496(\mathrm{~m}), 441$ (m).

HRMS (DART-TOF+) $m / z$ :

$[\mathrm{M}+\mathrm{H}]^{+}$Calcd for $\mathrm{C}_{13} \mathrm{H}_{18} \mathrm{NO}_{2} \mathrm{~S}^{+} 252.1053$;

Found 252.1053.

TLC (67\% EtOAc in hexanes), Rf:

$0.36(\mathrm{UV}, \mathrm{CAM})$.

M.p.:

$79-81{ }^{\circ} \mathrm{C}\left(\mathrm{CH}_{2} \mathrm{Cl}_{2}\right)$. 
<smiles>O=C(Cc1ccccc1)N1CCOCC1</smiles>

1a

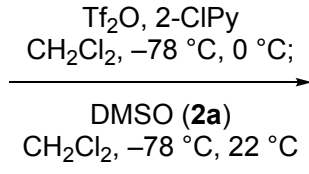

$\mathrm{CH}_{2} \mathrm{Cl}_{2},-78^{\circ} \mathrm{C}, 22^{\circ} \mathrm{C}$
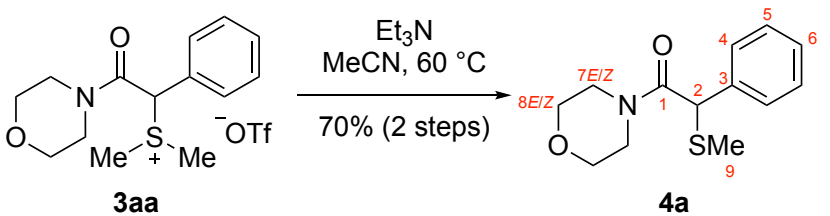

a-SMe-Morpholine Amide 4a (5.00 mmol scale):

Trifluoromethanesulfonic anhydride $(883 \mu \mathrm{L}, 5.25 \mathrm{mmol}, 1.05$ equiv) was added via syringe over $1 \mathrm{~min}$ to a stirred solution of azeotropically dried morpholine amide $1 \mathrm{a}$ (1.03 g, $5.00 \mathrm{mmol}, 1$ equiv) and 2-chloropyridine (1.42 $\mathrm{mL}, 15.0 \mathrm{mmol}, 3.00$ equiv) in dichloromethane $(17 \mathrm{~mL})$ at $-78^{\circ} \mathrm{C}$ under argon atmosphere. The dark red solution was stirred at $0{ }^{\circ} \mathrm{C}$ for $15 \mathrm{~min}$, before it was again cooled to $-78^{\circ} \mathrm{C}$. A solution of dimethyl sulfoxide $(\mathbf{2 a}, 426 \mu \mathrm{L}, 6.00 \mathrm{mmol}$, 1.20 equiv) in dichloromethane $(3.0 \mathrm{~mL})$ was added via cannula over $2 \mathrm{~min}$ at $-78^{\circ} \mathrm{C}$. The reaction mixture was stirred at $0{ }^{\circ} \mathrm{C}$ for $15 \mathrm{~min}$, then at $22^{\circ} \mathrm{C}$ for $30 \mathrm{~min}$ to give a yellow solution, which was concentrated under reduced pressure. The orange oily residue was dissolved in acetonitrile $(20 \mathrm{~mL})$. Triethylamine $(6.97 \mathrm{~mL}, 50.0 \mathrm{mmol}, 10.0$ equiv) was added, and the resulting brown solution was stirred in a sealed roundbottom flask at $60{ }^{\circ} \mathrm{C}$ for $15 \mathrm{~h}$. After concentration under reduced pressure, the brown oily crude material was purified via flash column chromatography on silica gel (eluent: 25\% EtOAc in hexanes) to yield $\alpha$-SMe-morpholine amide $4 \mathbf{a}(882 \mathrm{mg}, 70 \%)$ as a solid. The analytic data is fully consistent with that of compound 4a from Method A as described on a previous page of this document.

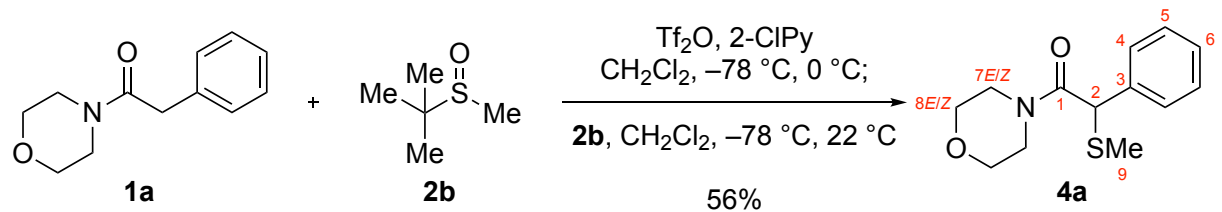

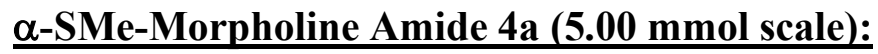

Trifluoromethanesulfonic anhydride $(883 \mu \mathrm{L}, 5.25 \mathrm{mmol}, 1.05$ equiv) was added via syringe over $1 \mathrm{~min}$ to a stirred solution of azeotropically dried morpholine amide $\mathbf{1 a}(1.03 \mathrm{~g}$, $5.00 \mathrm{mmol}, 1$ equiv) and 2-chloropyridine (1.42 mL, $15.0 \mathrm{mmol}, 3.00$ equiv) in dichloromethane $(17 \mathrm{~mL})$ at $-78^{\circ} \mathrm{C}$ under argon atmosphere. The dark red solution was stirred at $0{ }^{\circ} \mathrm{C}$ for $15 \mathrm{~min}$, before it was again cooled to $-78^{\circ} \mathrm{C}$. A solution of tert-butyl methyl sulfoxide $(2 \mathbf{b}, 698 \mu \mathrm{L}$, $6.00 \mathrm{mmol}, 1.20$ equiv) in dichloromethane $(3.0 \mathrm{~mL})$ was added via cannula over $2 \mathrm{~min}$ at $-78^{\circ} \mathrm{C}$. The reaction mixture was stirred at $0{ }^{\circ} \mathrm{C}$ for $15 \mathrm{~min}$, then at $22^{\circ} \mathrm{C}$ for $30 \mathrm{~min}$ to give a yellow solution. A saturated aqueous solution of sodium bicarbonate $(20 \mathrm{~mL})$ followed by dichloromethane $(10 \mathrm{~mL})$ was added, and the yellow biphasic mixture was vigorously stirred at $22{ }^{\circ} \mathrm{C}$ for $15 \mathrm{~min}$. After separation of the organic layer, the aqueous phase was extracted with dichloromethane $(3 \times 10 \mathrm{~mL})$. The combined light yellow organic extracts were dried over sodium sulfate, were filtered, and were concentrated under reduced pressure. The light yellow oily crude material was purified via flash column chromatography on silica gel (eluent: $25 \%$ EtOAc in hexanes) to yield $\alpha$-SMe-morpholine amide $\mathbf{4 a}(706 \mathrm{mg}, 56 \%)$ as a solid. The analytic data is fully consistent with that of compound 4a from Method A as described on a previous page of this document. 


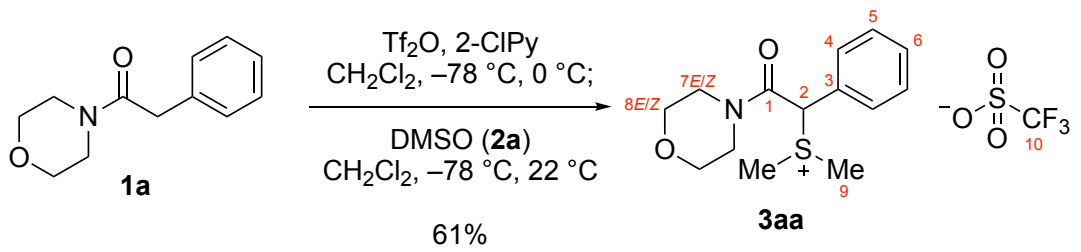

\section{Morpholine Amide Dimethyl Sulfonium Trifluoromethanesulfonate 3aa:}

Morpholine amide dimethyl sulfonium trifluoromethanesulfonate 3aa was prepared via Method A without triethylamine treatment for demethylation using azeotropically dried morpholine amide $\mathbf{1 a}(103 \mathrm{mg}, 500 \mu \mathrm{mol}, 1$ equiv) and dimethyl sulfoxide (2a, $42.6 \mu \mathrm{L}, 600 \mu \mathrm{mol}$, 1.20 equiv). The orange oily crude material was purified via flash column chromatography on silica gel (eluent: EtOAc $\rightarrow 10 \%$ methanol in EtOAc) to yield morpholine amide dimethyl sulfonium trifluoromethanesulfonate 3aa (127 mg, 61\%) as an oil. Structural assignments were made using additional information from gCOSY, gHSQC and gHMBC experiments.

${ }^{1} \mathrm{H}$ NMR $\left(500 \mathrm{MHz}, \mathrm{CDCl}_{3}, 30{ }^{\circ} \mathrm{C}\right)$ :

${ }^{13} \mathrm{C} \mathrm{NMR}\left(125 \mathrm{MHz}, \mathrm{CDCl}_{3}, 30{ }^{\circ} \mathrm{C}\right)$ :

${ }^{19} \mathrm{~F}$ NMR $\left(470 \mathrm{MHz}, \mathrm{CDCl}_{3}, 30{ }^{\circ} \mathrm{C}\right)$ :

FTIR (thin film) $\mathrm{cm}^{-1}$ :

HRMS (DART-TOF+) $m / z$ : $\delta$ 7.57-7.51 (m, 5H, $\left.2 \times \mathrm{C}_{4} \mathbf{H}, 2 \times \mathrm{C}_{5} \mathbf{H}, \mathrm{C}_{6} \mathbf{H}\right), 6.60(\mathrm{~s}$, $\left.1 \mathrm{H}, \mathrm{C}_{2} \mathbf{H}\right), 3.73-3.67\left(\mathrm{~m}, 1 \mathrm{H}, \mathrm{C}_{7 E / Z} \mathbf{H}\right), 3.66-3.63(\mathrm{~m}$, $\left.1 \mathrm{H}, \mathrm{C}_{8 E / Z} \mathbf{H}\right), 3.59-3.54\left(\mathrm{~m}, 3 \mathrm{H}, \mathrm{C}_{7 E / Z} \mathbf{H}, 2 \times \mathrm{C}_{8 E / Z} \mathbf{H}\right)$, 3.53-3.48 (m, 1H, $\left.\mathrm{C}_{7 E / Z} \mathbf{H}\right), 3.16-3.13(\mathrm{~m}, 1 \mathrm{H}$, $\left.\mathrm{C}_{8 E / Z} \mathbf{H}\right), 3.12-3.07\left(\mathrm{~m}, 1 \mathrm{H}, \mathrm{C}_{7 E / Z} \mathbf{H}\right), 3.10(\mathrm{~s}, 3 \mathrm{H}$, $\left.\mathrm{C}_{9} \mathbf{H}_{3}\right), 2.68$ (s, 3H, $\left.\mathrm{C}_{9} \mathbf{H}_{3}\right)$.

$\delta 163.6\left(\mathbf{C}_{\mathbf{1}}\right), 131.8\left(\mathbf{C}_{\mathbf{6}}\right), 130.7\left(2 \times \mathbf{C}_{\mathbf{5}}\right), 129.7(2 \times$ $\left.\mathbf{C}_{4}\right), 126.9\left(\mathbf{C}_{3}\right), 120.6\left(\mathrm{q}, J=320 \mathrm{~Hz}, \mathbf{C}_{\mathbf{1 0}}\right), 66.8\left(\mathbf{C}_{2}\right)$, $66.3\left(\mathbf{C}_{8 E / Z}\right), 66.1\left(\mathbf{C}_{8 E / Z}\right), 46.6\left(\mathbf{C}_{7 E / Z}\right), 43.3\left(\mathbf{C}_{7 E / Z}\right)$, $25.8\left(\mathbf{C}_{\mathbf{9}}\right), 22.1\left(\mathbf{C}_{\mathbf{9}}\right)$.

$\delta-79.4$.

3019 (w), 2858 (w), 1636 (s), 1459 (m), 1249 (s), 1153 (s), 1026 (s), 845 (w), 732 (m), 632 (s), 512 (m).

$[\mathrm{M}]^{+}$Calcd for $\mathrm{C}_{14} \mathrm{H}_{20} \mathrm{NO}_{2} \mathrm{~S}^{+}$266.1209;

Found 266.1213. 


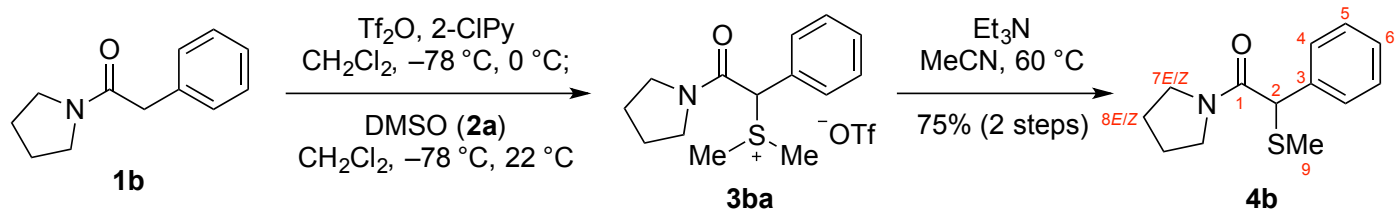

\section{a-SMe-Pyrrolidine Amide 4b:}

The $\alpha$-SMe-pyrrolidine amide $4 \mathbf{b}$ was prepared via Method A using azeotropically dried pyrrolidine amide $\mathbf{1 b}(94.6 \mathrm{mg}, 500 \mu \mathrm{mol}, 1$ equiv) and dimethyl sulfoxide (2a, $42.6 \mu \mathrm{L}, 600 \mu \mathrm{mol}$, 1.20 equiv). The brown oily crude material was purified via flash column chromatography on silica gel (eluent: $25 \%$ EtOAc in hexanes) to yield $\alpha$-SMe-pyrrolidine amide $\mathbf{4 b}(88.1 \mathrm{mg}, 75 \%)$ as a solid. Structural assignments were made using additional information from gCOSY, gHSQC and gHMBC experiments.
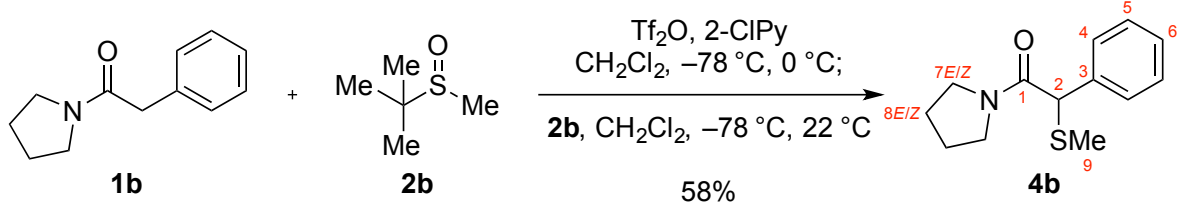

\section{a-SMe-Pyrrolidine Amide 4b:}

The $\alpha$-SMe-pyrrolidine amide $\mathbf{4 b}$ was prepared via Method B using azeotropically dried pyrrolidine amide $\mathbf{1 b}(94.6 \mathrm{mg}, 500 \mu \mathrm{mol}, 1$ equiv) and tert-butyl methyl sulfoxide $(\mathbf{2 b}, 69.8 \mu \mathrm{L}$, $600 \mu \mathrm{mol}, 1.20$ equiv). The light yellow oily crude material was purified via flash column chromatography on silica gel (eluent: $25 \%$ EtOAc in hexanes) to yield $\alpha$-SMe-pyrrolidine amide $\mathbf{4 b}(68.3 \mathrm{mg}, 58 \%)$ as a solid. The analytic data is fully consistent with that of compound $\mathbf{4 b}$ from Method A.

${ }^{1} \mathrm{H}$ NMR $\left(400 \mathrm{MHz}, \mathrm{CDCl}_{3}, 25^{\circ} \mathrm{C}\right)$ :

${ }^{13} \mathrm{C}$ NMR (100 MHz, $\left.\mathrm{CDCl}_{3}, 25{ }^{\circ} \mathrm{C}\right)$ :

FTIR (thin film) $\mathrm{cm}^{-1}$ :

HRMS (DART-TOF+) $m / z$ :

TLC (67\% EtOAc in hexanes), Rf:

M.p.: $\delta 7.45\left(\mathrm{~d}, J=7.3 \mathrm{~Hz}, 2 \mathrm{H}, 2 \times \mathrm{C}_{4} \mathbf{H}\right), 7.33(\mathrm{t}, J=$ $\left.7.3 \mathrm{~Hz}, 2 \mathrm{H}, 2 \times \mathrm{C}_{5} \mathbf{H}\right), 7.29-7.25\left(\mathrm{~m}, 1 \mathrm{H}, \mathrm{C}_{6} \mathbf{H}\right), 4.58$ (s, 1H, $\left.\mathrm{C}_{2} \mathbf{H}\right), 3.59-3.49\left(\mathrm{~m}, 2 \mathrm{H}, 2 \times \mathrm{C}_{7 E / Z} \mathbf{H}\right), 3.47-$ $3.40\left(\mathrm{~m}, 1 \mathrm{H}, \mathrm{C}_{7 E / Z} \mathbf{H}\right), 3.29-3.23\left(\mathrm{~m}, 1 \mathrm{H}, \mathrm{C}_{7 E / Z} \mathbf{H}\right)$, 2.00 (s, 3H, $\left.\mathrm{C}_{9} \mathbf{H}_{3}\right), 1.95-1.89\left(\mathrm{~m}, 1 \mathrm{H}, \mathrm{C}_{8 E / Z} \mathbf{H}\right), 1.87-$ $1.75\left(\mathrm{~m}, 3 \mathrm{H}, 3 \times \mathrm{C}_{8 E / Z} \mathbf{H}\right)$.

$\delta 168.1\left(\mathbf{C}_{\mathbf{1}}\right), 136.7\left(\mathbf{C}_{3}\right), 128.8\left(2 \times \mathbf{C}_{4}\right), 128.7(2 \times$ $\left.\mathbf{C}_{5}\right), 127.9\left(\mathbf{C}_{6}\right), 54.2\left(\mathbf{C}_{2}\right), 46.7\left(\mathbf{C}_{7 E / Z}\right), 46.5\left(\mathbf{C}_{7 E / Z}\right)$, 26.3 ( $\left.\mathbf{C}_{8 E / Z}\right), 24.3\left(\mathbf{C}_{8 E / Z}\right), 14.5\left(\mathbf{C}_{9}\right)$.

2976 (w), $2911(\mathrm{w}), 2872(\mathrm{w}), 1629$ (s), 1421 (s), 1253 (w), $1187(\mathrm{w}), 861(\mathrm{w}), 746(\mathrm{~m}), 696(\mathrm{~s}), 676$ (m), 508 (m).

$[\mathrm{M}+\mathrm{H}]^{+}$Calcd for $\mathrm{C}_{13} \mathrm{H}_{18} \mathrm{NOS}^{+} 236.1104$;

Found 236.1104.

0.42 (UV, CAM).

$75-77{ }^{\circ} \mathrm{C}\left(\mathrm{CH}_{2} \mathrm{Cl}_{2}\right)$. 


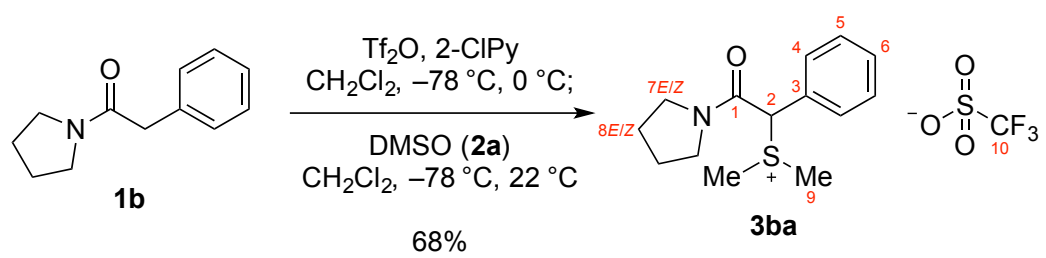

\section{Pyrrolidine Amide Dimethyl Sulfonium Trifluoromethanesulfonate 3ba:}

Pyrrolidine amide dimethyl sulfonium trifluoromethanesulfonate $\mathbf{3 b a}$ was prepared via Method A without triethylamine treatment for demethylation using azeotropically dried pyrrolidine amide $\mathbf{1 b}$ (94.6 mg, $500 \mu \mathrm{mol}, 1$ equiv) and dimethyl sulfoxide (2a, $42.6 \mu \mathrm{L}, 600 \mu \mathrm{mol}$, 1.20 equiv). The orange oily crude material was purified via flash column chromatography on silica gel (eluent: EtOAc $\rightarrow 5 \%$ methanol in EtOAc) to yield pyrrolidine amide dimethyl sulfonium trifluoromethanesulfonate $\mathbf{3 b a}(136 \mathrm{mg}, 68 \%)$ as an oil. Structural assignments were made using additional information from gCOSY, gHSQC and gHMBC experiments.

${ }^{1} \mathrm{H}$ NMR (400 $\left.\mathrm{MHz}, \mathrm{CDCl}_{3}, 25^{\circ} \mathrm{C}\right)$ :

${ }^{13} \mathrm{C}$ NMR (100 MHz, $\left.\mathrm{CDCl}_{3}, 25^{\circ} \mathrm{C}\right)$ :

${ }^{19} \mathrm{~F}$ NMR $\left(376 \mathrm{MHz}, \mathrm{CDCl}_{3}, 25^{\circ} \mathrm{C}\right)$ :

FTIR (thin film) $\mathrm{cm}^{-1}$ :

HRMS (DART-TOF+) $m / z$ : $\delta$ 7.54-7.52 (m, 2H, $\left.2 \times \mathrm{C}_{4} \mathbf{H}\right), 7.50-7.44(\mathrm{~m}, 3 \mathrm{H}, 2 \times$ $\left.\mathrm{C}_{5} \mathbf{H}, \mathrm{C}_{6} \mathbf{H}\right), 6.24\left(\mathrm{~s}, 1 \mathrm{H}, \mathrm{C}_{2} \mathbf{H}\right), 3.74-3.68(\mathrm{~m}, 1 \mathrm{H}$, $\left.\mathrm{C}_{7 E / Z} \mathbf{H}\right), 3.51\left(\mathrm{dt}, J=13.4,6.9 \mathrm{~Hz}, 1 \mathrm{H}, \mathrm{C}_{7 E / Z} \mathbf{H}\right), 3.40$ $\left(\mathrm{dt}, J=12.4,6.7 \mathrm{~Hz}, 1 \mathrm{H}, \mathrm{C}_{7 E / Z} \mathbf{H}\right), 3.06\left(\mathrm{~s}, 3 \mathrm{H}, \mathrm{C}_{9} \mathbf{H}_{3}\right)$, $2.74\left(\mathrm{dt}, J=10.7,6.9 \mathrm{~Hz}, 1 \mathrm{H}, \mathrm{C}_{7 E / Z} \mathbf{H}\right), 2.63(\mathrm{~s}, 3 \mathrm{H}$, $\left.\mathrm{C}_{9} \mathbf{H}_{3}\right), 1.95-1.81\left(\mathrm{~m}, 2 \mathrm{H}, 2 \times \mathrm{C}_{8 E / Z} \mathbf{H}\right), 1.78-1.64(\mathrm{~m}$, $\left.2 \mathrm{H}, 2 \times \mathrm{C}_{8 E / Z} \mathbf{H}\right)$.

$\delta 163.0\left(\mathbf{C}_{\mathbf{1}}\right), 131.5\left(\mathbf{C}_{\mathbf{6}}\right), 130.4\left(2 \times \mathbf{C}_{\mathbf{5}}\right), 129.9(2 \times$ $\left.\mathbf{C}_{4}\right), 126.6\left(\mathbf{C}_{3}\right), 120.5\left(\mathrm{q}, J=320 \mathrm{~Hz}, \mathbf{C}_{\mathbf{1 0}}\right), 67.0\left(\mathbf{C}_{2}\right)$, $47.1\left(\mathbf{C}_{7 E / Z}\right), 46.6\left(\mathbf{C}_{7 E / Z}\right), 25.9\left(\mathbf{C}_{8 E / Z}\right), 25.6\left(\mathbf{C}_{9}\right)$, $24.0\left(\mathbf{C}_{\mathbf{8 E} / Z}\right), 21.9\left(\mathbf{C}_{\mathbf{9}}\right)$.

$\delta-79.4$.

$2936(\mathrm{w}), 1635(\mathrm{~s}), 1440(\mathrm{~m}), 1248(\mathrm{~s}), 1151(\mathrm{~s}), 1027$ (s), $716(\mathrm{~m}), 635(\mathrm{~s}), 516(\mathrm{~m})$.

$[\mathrm{M}]^{+}$Calcd for $\mathrm{C}_{14} \mathrm{H}_{20} \mathrm{NOS}^{+} 250.1260$;

Found 250.1256. 


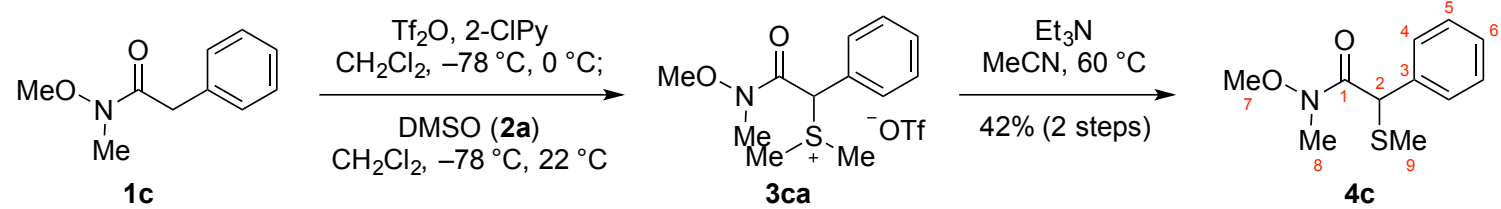

\section{$\alpha$-SMe-Weinreb Amide 4c:}

The $\alpha$-SMe-Weinreb amide 4c was prepared via Method A using azeotropically dried Weinreb amide 1c $(89.6 \mathrm{mg}, 500 \mu \mathrm{mol}, 1$ equiv) and dimethyl sulfoxide $(\mathbf{2 a}, 42.6 \mu \mathrm{L}, 600 \mu \mathrm{mol}$, 1.20 equiv). The brown oily crude material was purified via flash column chromatography on silica gel (eluent: $25 \%$ EtOAc in hexanes) to yield $\alpha$-SMe-Weinreb amide $4 \mathrm{c}$ (47.0 $\mathrm{mg}, 42 \%$ ) as a solid. Structural assignments were made using additional information from gCOSY, gHSQC and gHMBC experiments.

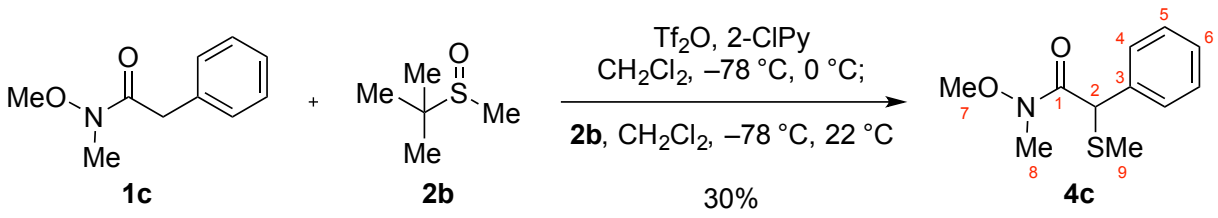

$\alpha$-SMe-Weinreb Amide 4c:

The $\alpha$-SMe-Weinreb amide 4c was prepared via Method B using azeotropically dried Weinreb amide 1c $(89.6 \mathrm{mg}, 500 \mu \mathrm{mol}, 1$ equiv) and tert-butyl methyl sulfoxide $(\mathbf{2 b}, 69.8 \mu \mathrm{L}$, $600 \mu \mathrm{mol}, 1.20$ equiv). The light yellow oily crude material was purified via flash column chromatography on silica gel (eluent: $25 \%$ EtOAc in hexanes) to yield $\alpha$-SMe-Weinreb amide $4 \mathbf{c}$ $(33.8 \mathrm{mg}, 30 \%)$ as a solid. The analytic data is fully consistent with that of compound $\mathbf{4 c}$ from Method A.

${ }^{1} \mathrm{H}$ NMR $\left(400 \mathrm{MHz}, \mathrm{CDCl}_{3}, 25^{\circ} \mathrm{C}\right)$ :

${ }^{13} \mathrm{C}$ NMR $\left(100 \mathrm{MHz}, \mathrm{CDCl}_{3}, 25{ }^{\circ} \mathrm{C}\right)$ :

FTIR (thin film) $\mathrm{cm}^{-1}$ :

HRMS (DART-TOF+) $m / z$ :

TLC (33\% EtOAc in hexanes), Rf:

M.p.: $\delta$ 7.47-7.45 (m, 2H, $\left.2 \times \mathrm{C}_{4} \mathbf{H}\right), 7.35-7.31(\mathrm{~m}, 2 \mathrm{H}, 2 \times$ $\left.\mathrm{C}_{5} \mathbf{H}\right), 7.29-7.25\left(\mathrm{~m}, 1 \mathrm{H}, \mathrm{C}_{6} \mathbf{H}\right), 5.02\left(\mathrm{~s}, 1 \mathrm{H}, \mathrm{C}_{2} \mathbf{H}\right)$, $3.48\left(\mathrm{~s}, 3 \mathrm{H}, \mathrm{C}_{7} \mathbf{H}_{3}\right), 3.19\left(\mathrm{~s}, 3 \mathrm{H}, \mathrm{C}_{8} \mathbf{H}_{3}\right), 2.03(\mathrm{~s}, 3 \mathrm{H}$, $\left.\mathrm{C}_{9} \mathrm{H}_{3}\right)$.

$\delta 171.1\left(\mathbf{C}_{\mathbf{1}}\right), 136.9\left(\mathbf{C}_{3}\right), 128.8\left(2 \times \mathbf{C}_{4}\right), 128.7(2 \times$ $\left.\mathbf{C}_{5}\right), 127.9\left(\mathbf{C}_{6}\right), 61.5\left(\mathbf{C}_{7}\right), 50.9\left(\mathbf{C}_{2}\right), 32.6\left(\mathbf{C}_{8}\right), 14.5$ (C9).

2915 (w), 1644 (s), 1410 (m), 1379 (s), 1167 (m), 987 (s), $733(\mathrm{~s}), 690(\mathrm{~s}), 622(\mathrm{~m}), 495(\mathrm{~m}), 476(\mathrm{~m})$.

$[\mathrm{M}+\mathrm{H}]^{+}$Calcd for $\mathrm{C}_{11} \mathrm{H}_{16} \mathrm{NO}_{2} \mathrm{~S}^{+}$226.0896; Found 226.0892.

0.38 (UV, CAM). $32-34{ }^{\circ} \mathrm{C}\left(\mathrm{CH}_{2} \mathrm{Cl}_{2}\right)$. 


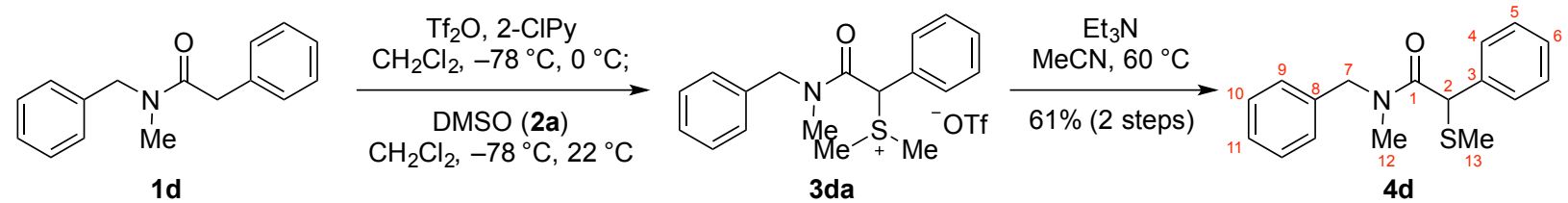

$\alpha$-SMe- $N$-Benzyl- $N$-Methyl Amide 4d:

The $\alpha$-SMe- $N$-benzyl- $N$-methyl amide 4d was prepared via Method A using azeotropically dried $N$-benzyl- $N$-methyl amide 1d (120 mg, $500 \mu \mathrm{mol}, 1$ equiv) and dimethyl sulfoxide (2a, $42.6 \mu \mathrm{L}, 600 \mu \mathrm{mol}, 1.20$ equiv). The brown oily crude material was purified via flash column chromatography on silica gel (eluent: $15 \%$ EtOAc in hexanes) to yield $\alpha$-SMe- $N$-benzyl- $N$-methyl amide 4d (86.9 mg, 61\%) as an oil. ${ }^{7,8}$ Structural assignments were made using additional information from gCOSY, gHSQC and gHMBC experiments.
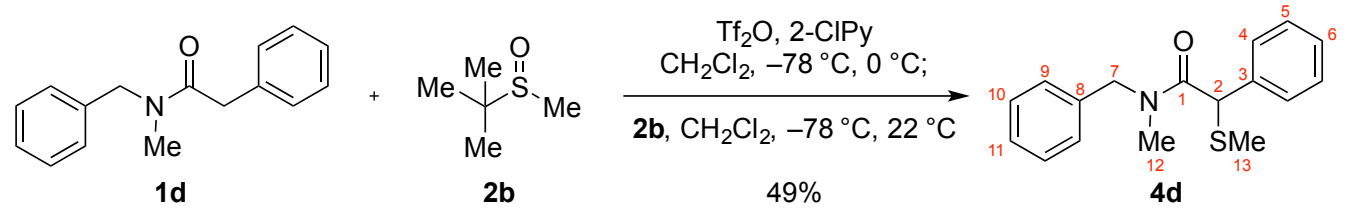

\section{$\alpha$-SMe- $N$-Benzyl- $N$-Methyl Amide 4d:}

The $\alpha$-SMe- $N$-benzyl- $N$-methyl amide 4d was prepared via Method B using azeotropically dried $N$-benzyl- $N$-methyl amide 1d $(120 \mathrm{mg}, 500 \mu \mathrm{mol}, 1$ equiv) and tert-butyl methyl sulfoxide (2b, $69.8 \mu \mathrm{L}, 600 \mu \mathrm{mol}, 1.20$ equiv). The light yellow oily crude material was purified via flash column chromatography on silica gel (eluent: $15 \%$ EtOAc in hexanes) to yield $\alpha$-SMe- $N$-benzyl$\mathrm{N}$-methyl amide $\mathbf{4 d}(70.5 \mathrm{mg}, 49 \%)$ as an oil. ${ }^{7,8}$ The analytic data is fully consistent with that of compound 4d from Method A.

${ }^{1} \mathrm{H} \mathrm{NMR}\left(400 \mathrm{MHz}, \mathrm{CDCl}_{3}, 25^{\circ} \mathrm{C}, 3: 2\right.$ mixture of atropisomers, *denotes minor atropisomers):

$\delta 7.45\left(\mathrm{~d}, J=7.4 \mathrm{~Hz}, 2 \mathrm{H}, 2 \times \mathrm{C}_{4} \mathbf{H}\right), 7.40(\mathrm{~d}, J=$ $\left.7.2 \mathrm{~Hz}, 2 \mathrm{H}, 2 \times \mathrm{C}_{4} \mathbf{H}^{*}\right), 7.37-7.22\left(\mathrm{~m}, 6 \mathrm{H}, 6 \times \mathrm{C}_{\mathrm{Ar}} \mathbf{H}\right.$, $\left.6 \mathrm{H}^{*}, 6 \times \mathrm{C}_{\mathrm{Ar}} \mathbf{H}^{*}\right), 7.19\left(\mathrm{~d}, J=8.0 \mathrm{~Hz}, 2 \mathrm{H}, 2 \times \mathrm{C}_{\mathrm{Ar}} \mathbf{H}\right)$, $7.06\left(\mathrm{~d}, J=7.3 \mathrm{~Hz}, 2 \mathrm{H}, 2 \times \mathrm{C}_{\mathrm{Ar}} \mathbf{H}^{*}\right), 4.78(\mathrm{~s}, 1 \mathrm{H}$, $\left.\mathrm{C}_{2} \mathbf{H}\right), 4.70$ (s, 1H, $\left.\mathrm{C}_{2} \mathbf{H}^{*}\right), 4.65-4.57\left(\mathrm{~m}, 2 \mathrm{H}, \mathrm{C}_{7} \mathbf{H}_{2}\right.$, $\left.1 \mathrm{H}, \mathrm{C}_{7} \mathbf{H}_{\mathbf{a}}{ }^{*}\right), 4.47$ (d, $\left.J=16.9 \mathrm{~Hz}, 1 \mathrm{H}, \mathrm{C}_{7} \mathbf{H}_{\mathbf{b}} *\right), 2.97$ $\left(\mathrm{s}, 3 \mathrm{H}, \mathrm{C}_{12} \mathbf{H}_{3}{ }^{*}\right), 2.87\left(\mathrm{~s}, 3 \mathrm{H}, \mathrm{C}_{12} \mathbf{H}_{3}\right), 2.03(\mathrm{~s}, 3 \mathrm{H}$, $\left.\mathrm{C}_{13} \mathbf{H}_{3}\right), 1.96\left(\mathrm{~s}, 3 \mathrm{H}, \mathrm{C}_{13} \mathbf{H}_{3} *\right)$.

${ }^{13} \mathrm{C} \mathrm{NMR}\left(100 \mathrm{MHz}, \mathrm{CDCl}_{3}, 25^{\circ} \mathrm{C}, 3: 2\right.$ mixture of atropisomers, *denotes minor atropisomers): $\delta 170.1\left(\mathbf{C}_{\mathbf{1}}{ }^{*}\right), 169.8\left(\mathbf{C}_{1}\right), 137.0\left(\mathbf{C}_{3}\right), 136.9\left(\mathbf{C}_{3}{ }^{*}\right)$, $136.6\left(\mathbf{C}_{8}\right), 136.3\left(\mathbf{C}_{8}{ }^{*}\right), 129.0\left(\mathbf{C}_{\mathbf{A r}}{ }^{*}\right), 128.8\left(\mathbf{C}_{\mathbf{A r}}{ }^{*}\right)$, $128.7\left(\mathbf{C}_{\mathrm{Ar}}\right), 128.6\left(\mathbf{C}_{\mathrm{Ar}}\right), 128.5\left(\mathbf{C}_{\mathrm{Ar}}\right), 128.5\left(\mathbf{C}_{\mathrm{Ar}}{ }^{*}\right)$, $128.0\left(\mathbf{C}_{\mathbf{A r}}\right), 127.9\left(\mathbf{C}_{\mathbf{A r}}{ }^{*}\right), 127.9\left(\mathbf{C}_{\mathbf{A r}}\right), 127.7\left(\mathbf{C}_{\mathbf{A r}}{ }^{*}\right)$, $127.4\left(\mathbf{C}_{\mathrm{Ar}}\right), 126.3\left(\mathbf{C}_{\mathbf{A r}}{ }^{*}\right), 53.4\left(\mathbf{C}_{7}{ }^{*}\right), 53.4\left(\mathbf{C}_{2}\right), 53.0$ $\left(\mathbf{C}_{2} *\right), 51.5\left(\mathbf{C}_{7}\right), 35.0\left(\mathbf{C}_{12}\right), 34.6\left(\mathbf{C}_{12} *\right), 14.7\left(\mathbf{C}_{13} *\right)$, $14.5\left(\mathbf{C}_{13}\right)$.

FTIR (thin film) $\mathrm{cm}^{-1}$ : 1355 (w), $1104(\mathrm{~m}), 1029(\mathrm{w}), 733(\mathrm{~m}), 695$ (s), 511 (m). 
HRMS (DART-TOF+) $m / z$ :

TLC (33\% EtOAc in hexanes), Rf:
$[\mathrm{M}+\mathrm{H}]^{+}$Calcd for $\mathrm{C}_{17} \mathrm{H}_{20} \mathrm{NOS}^{+} 286.1260$

Found 286.1256.

0.45 (UV, CAM). 


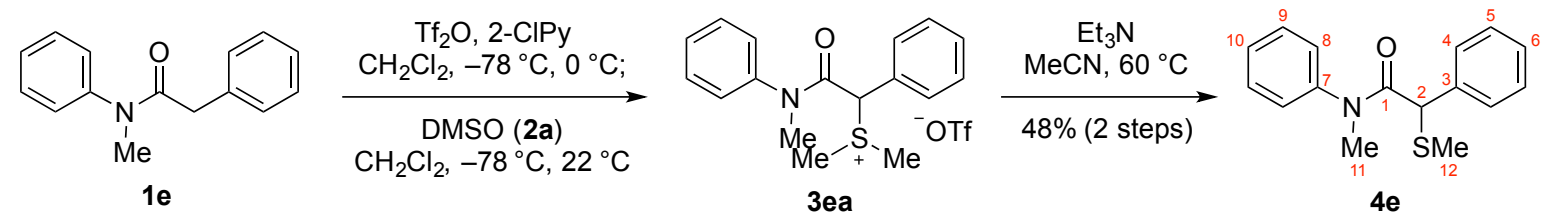

$\underline{\alpha-S M e-N \text {-Phenyl- } N \text {-Methyl Amide 4e: }}$

The $\alpha$-SMe- $N$-phenyl- $N$-methyl amide 4e was prepared via Method A using azeotropically dried $N$-phenyl- $N$-methyl amide 1e $(113 \mathrm{mg}, 500 \mu \mathrm{mol}, 1$ equiv) and dimethyl sulfoxide (2a, $42.6 \mu \mathrm{L}, 600 \mu \mathrm{mol}, 1.20$ equiv). The brown oily crude material was purified via flash column chromatography on silica gel (eluent: $10 \%$ EtOAc in hexanes) to yield $\alpha$-SMe- $N$-phenyl- $N$-methyl amide $4 \mathbf{e}(65.1 \mathrm{mg}, 48 \%)$ as an oil. ${ }^{7,9}$ Structural assignments were made using additional information from gCOSY, gHSQC and gHMBC experiments.
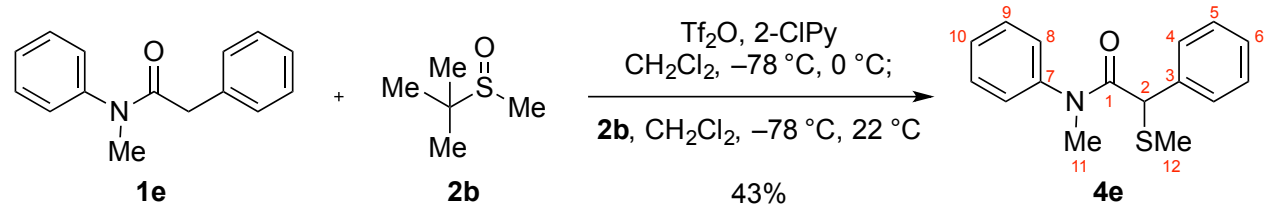

\section{$\alpha$-SMe- $N$-Phenyl- $N$-Methyl Amide 4e:}

The $\alpha$-SMe- $N$-phenyl- $N$-methyl amide 4e was prepared via Method B using azeotropically dried $N$-phenyl- $N$-methyl amide 1e $(113 \mathrm{mg}, 500 \mu \mathrm{mol}, 1$ equiv) and tert-butyl methyl sulfoxide (2b, $69.8 \mu \mathrm{L}, 600 \mu \mathrm{mol}, 1.20$ equiv). The light yellow oily crude material was purified via flash column chromatography on silica gel (eluent: $10 \%$ EtOAc in hexanes) to yield $\alpha$-SMe- $N$-phenyl$\mathrm{N}$-methyl amide $4 \mathbf{e}(58.3 \mathrm{mg}, 43 \%)$ as an oil. ${ }^{7,10}$ The analytic data is fully consistent with that of compound $4 \mathbf{e}$ from Method A.

${ }^{1} \mathrm{H} \mathrm{NMR}\left(400 \mathrm{MHz}, \mathrm{CDCl}_{3}, 25{ }^{\circ} \mathrm{C}\right)$ :

${ }^{13} \mathrm{C}$ NMR $\left(100 \mathrm{MHz}, \mathrm{CDCl}_{3}, 25{ }^{\circ} \mathrm{C}\right)$ :

FTIR (thin film) $\mathrm{cm}^{-1}$ :

HRMS (DART-TOF+) $m / z$ :

TLC (33\% EtOAc in hexanes), Rf: $\delta$ 7.41-7.36 (m, 3H, $\left.2 \times \mathrm{C}_{4} \mathbf{H}, \mathrm{C}_{6} \mathbf{H}\right), 7.26-7.20(\mathrm{~m}$, $\left.5 \mathrm{H}, 2 \times \mathrm{C}_{5} \mathbf{H}, 2 \times \mathrm{C}_{9} \mathbf{H}, \mathrm{C}_{10} \mathbf{H}\right), 7.08\left(\mathrm{bs}, 2 \mathrm{H}, 2 \times \mathrm{C}_{8} \mathbf{H}\right)$, $4.40\left(\mathrm{~s}, 1 \mathrm{H}, \mathrm{C}_{2} \mathbf{H}\right), 3.28\left(\mathrm{~s}, 3 \mathrm{H}, \mathrm{C}_{11} \mathbf{H}_{3}\right), 1.91(\mathrm{~s}, 3 \mathrm{H}$, $\mathrm{C}_{12} \mathbf{H}_{3}$ ).

$\delta 170.0\left(\mathbf{C}_{\mathbf{1}}\right), 143.5\left(\mathbf{C}_{7}\right), 137.0\left(\mathbf{C}_{\mathbf{3}}\right), 129.9\left(2 \times \mathbf{C}_{5}\right)$, $128.7\left(2 \times \mathbf{C}_{4}\right), 128.5\left(2 \times \mathbf{C}_{9}\right), 128.3\left(\mathbf{C}_{6}\right), 127.8(2 \times$ $\left.\mathbf{C}_{8}, \mathbf{C}_{10}\right), 51.8\left(\mathbf{C}_{2}\right), 38.0\left(\mathbf{C}_{11}\right), 14.5\left(\mathbf{C}_{12}\right)$.

3060 (w), 2914 (w), 1652 (s), 1593 (m), 1493 (s), 1372 (s), 1292 (m), $1238(\mathrm{w}), 1114(\mathrm{~s}), 772(\mathrm{~m}), 735$ (m), $695(\mathrm{~s}), 661(\mathrm{~m}), 553(\mathrm{~m}), 498(\mathrm{~m})$.

$[\mathrm{M}+\mathrm{H}]^{+}$Calcd for $\mathrm{C}_{16} \mathrm{H}_{18} \mathrm{NOS}^{+} 272.1104$; Found 272.1099.

0.51 (UV, CAM). 


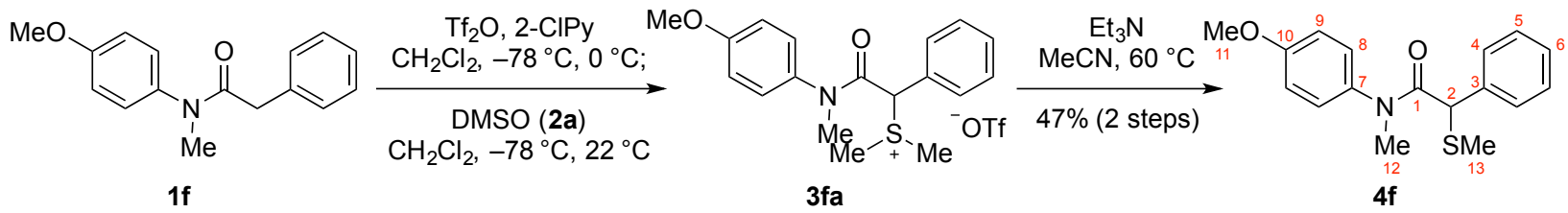

\section{$\alpha$-SMe- $N$-Phenyl- $N$-Methyl Amide 4f:}

The $\alpha$-SMe- $N$-phenyl- $N$-methyl amide $4 \mathbf{f}$ was prepared via Method A using azeotropically dried $N$-phenyl- $N$-methyl amide $\mathbf{1 f}(128 \mathrm{mg}, 500 \mu \mathrm{mol}, 1$ equiv) and dimethyl sulfoxide (2a, $42.6 \mu \mathrm{L}, 600 \mu \mathrm{mol}, 1.20$ equiv). The brown oily crude material was purified via flash column chromatography on silica gel (eluent: $20 \%$ EtOAc in hexanes) to yield $\alpha$-SMe- $N$-phenyl- $N$-methyl amide $4 \mathrm{f}(70.7 \mathrm{mg}, 47 \%)$ as a solid. ${ }^{7,11}$ Structural assignments were made using additional information from gCOSY, gHSQC and gHMBC experiments.
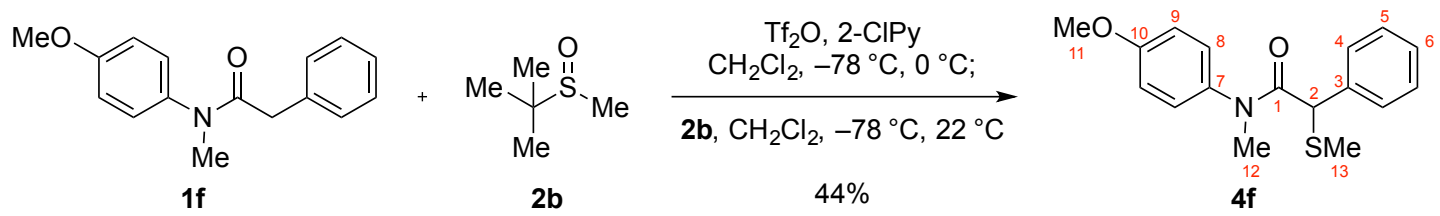

$\alpha$-SMe- $N$-Phenyl- $N$-Methyl Amide 4f:

The $\alpha$-SMe- $N$-phenyl- $N$-methyl amide $4 \mathbf{f}$ was prepared via Method B using azeotropically dried $N$-phenyl- $N$-methyl amide $\mathbf{1 f}(128 \mathrm{mg}, 500 \mu \mathrm{mol}, 1$ equiv) and tert-butyl methyl sulfoxide (2b, $69.8 \mu \mathrm{L}, 600 \mu \mathrm{mol}, 1.20$ equiv). The bright orange oily crude material was purified via flash column chromatography on silica gel (eluent: 20\% EtOAc in hexanes) to yield $\alpha$-SMe- $N$-phenyl$N$-methyl amide $\mathbf{4 f}(66.3 \mathrm{mg}, 44 \%)$ as a solid. ${ }^{7,12}$ The analytic data is fully consistent with that of compound 4f from Method A.

${ }^{1} \mathrm{H}$ NMR $\left(400 \mathrm{MHz}, \mathrm{CDCl}_{3}, 25^{\circ} \mathrm{C}\right)$ :

${ }^{13} \mathrm{C}$ NMR (100 MHz, $\left.\mathrm{CDCl}_{3}, 25^{\circ} \mathrm{C}\right)$ :

FTIR (thin film) $\mathrm{cm}^{-1}$ :

HRMS (DART-TOF+) $m / z$ :

TLC (67\% EtOAc in hexanes), Rf:

M.p.: $\delta 7.30-7.23\left(\mathrm{~m}, 5 \mathrm{H}, 2 \times \mathrm{C}_{4} \mathbf{H}, 2 \times \mathrm{C}_{5} \mathbf{H}, \mathrm{C}_{6} \mathbf{H}\right), 7.00$ (bs, $\left.2 \mathrm{H}, 2 \times \mathrm{C}_{8} \mathbf{H}\right), 6.89\left(\mathrm{~d}, J=8.2 \mathrm{~Hz}, 2 \mathrm{H}, 2 \times \mathrm{C}_{9} \mathbf{H}\right)$, $4.44\left(\mathrm{~s}, 1 \mathrm{H}, \mathrm{C}_{2} \mathbf{H}\right), 3.86\left(\mathrm{~s}, 3 \mathrm{H}, \mathrm{C}_{11} \mathbf{H}_{3}\right), 3.26(\mathrm{~s}, 3 \mathrm{H}$, $\left.\mathrm{C}_{12} \mathbf{H}_{3}\right), 1.93$ (s, 3H, $\left.\mathrm{C}_{13} \mathbf{H}_{3}\right)$.

$\delta 170.3\left(\mathbf{C}_{\mathbf{1}}\right), 159.3\left(\mathbf{C}_{\mathbf{1 0}}\right), 137.1\left(\mathbf{C}_{\mathbf{3}}\right), 136.2\left(\mathbf{C}_{7}\right)$, $128.8\left(2 \times \mathbf{C}_{8}\right), 128.8\left(2 \times \mathbf{C}_{4}\right), 128.4\left(2 \times \mathbf{C}_{5}\right), 127.7$ $\left(\mathbf{C}_{6}\right), 114.9\left(2 \times \mathbf{C}_{9}\right), 55.6\left(\mathbf{C}_{11}\right), 51.7\left(\mathbf{C}_{2}\right), 38.1\left(\mathbf{C}_{12}\right)$, $14.6\left(\mathbf{C}_{13}\right)$.

2955 (w), 2921 (w), 2837 (w), 1650 (s), 1504 (s), $1453(\mathrm{~m}), 1369(\mathrm{~m}), 1240$ (s), $1106(\mathrm{~m}), 1024(\mathrm{~m})$, 845 (m), 735 (s), 699 (s), 605 (m), 564 (s), 502 (m).

$[\mathrm{M}+\mathrm{H}]^{+}$Calcd for $\mathrm{C}_{17} \mathrm{H}_{20} \mathrm{NO}_{2} \mathrm{~S}^{+} 302.1209$;

Found 302.1205.

0.67 (UV, CAM).

97-99 ${ }^{\circ} \mathrm{C}\left(\mathrm{CH}_{2} \mathrm{Cl}_{2}\right)$. 


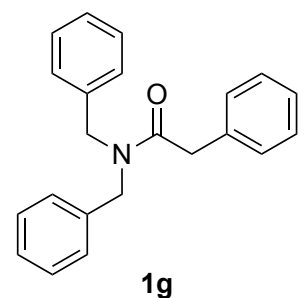

$1 \mathrm{~g}$

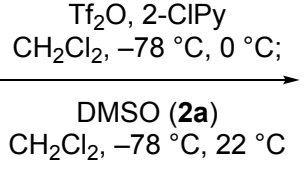

$\mathrm{CH}_{2} \mathrm{Cl}_{2},-78^{\circ} \mathrm{C}, 22^{\circ} \mathrm{C}$

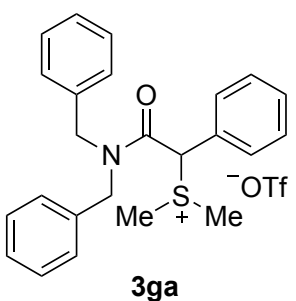

3ga

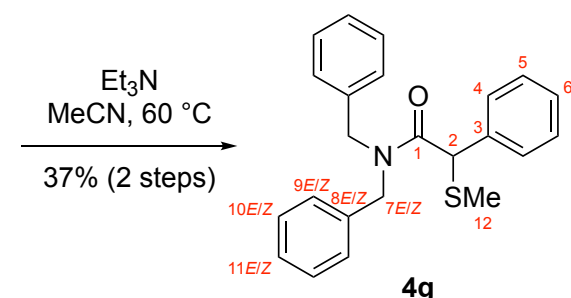

$4 \mathrm{~g}$

\section{$\alpha$-SMe- $N, N$-Dibenzyl Amide 4g:}

The $\alpha$-SMe- $N, N$-dibenzyl amide $\mathbf{4 g}$ was prepared via Method A using azeotropically dried $N, N$-dibenzyl amide $\mathbf{1 g}(158 \mathrm{mg}, 500 \mu \mathrm{mol}, 1$ equiv) and dimethyl sulfoxide $(\mathbf{2 a}, 42.6 \mu \mathrm{L}$, $600 \mu \mathrm{mol}, 1.20$ equiv). The brown oily crude material was purified via flash column chromatography on silica gel (eluent: $10 \%$ EtOAc in hexanes) to yield $\alpha$-SMe- $N, N$-dibenzyl amide $\mathbf{4 g}(66.2 \mathrm{mg}, 37 \%)$ as an oil. ${ }^{7,13}$ Structural assignments were made using additional information from gCOSY, gHSQC and gHMBC experiments.
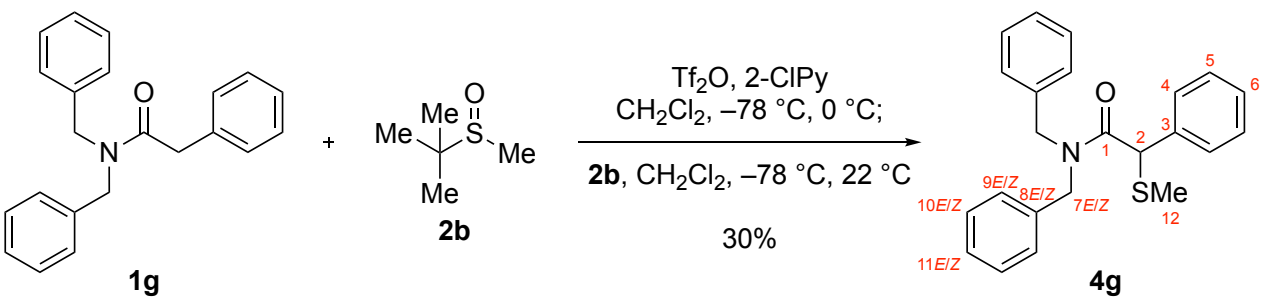

\section{Q-SMe- $N, N$-Dibenzyl Amide 4g:}

The $\alpha$-SMe- $N, N$-dibenzyl amide $4 \mathrm{~g}$ was prepared via Method B using azeotropically dried $N, N$-dibenzyl amide $\mathbf{1 g}(158 \mathrm{mg}, 500 \mu \mathrm{mol}, 1$ equiv) and tert-butyl methyl sulfoxide $(\mathbf{2 b}, 69.8 \mu \mathrm{L}$, $600 \mu \mathrm{mol}, 1.20$ equiv). The light yellow oily crude material was purified via flash column chromatography on silica gel (eluent: $10 \%$ EtOAc in hexanes) to yield $\alpha$-SMe- $N, N$-dibenzyl amide $\mathbf{4 g}(54.4 \mathrm{mg}, 30 \%)$ as an oil. ${ }^{7,14}$ The analytic data is fully consistent with that of compound $\mathbf{4 g}$ from Method A.

${ }^{1} \mathrm{H}$ NMR $\left(400 \mathrm{MHz}, \mathrm{CDCl}_{3}, 25^{\circ} \mathrm{C}\right)$ :

${ }^{13} \mathrm{C} \mathrm{NMR}\left(100 \mathrm{MHz}, \mathrm{CDCl}_{3}, 25{ }^{\circ} \mathrm{C}\right)$ :

FTIR (thin film) $\mathrm{cm}^{-1}$ : $\delta 7.44\left(\mathrm{~d}, J=6.6 \mathrm{~Hz}, 2 \mathrm{H}, 2 \times \mathrm{C}_{4} \mathbf{H}\right), 7.40-7.26(\mathrm{~m}$, $\left.9 \mathrm{H}, 2 \times \mathrm{C}_{5} \mathbf{H}, \mathrm{C}_{6} \mathbf{H}, 4 \times \mathrm{C}_{10 E / Z} \mathbf{H}, 2 \times \mathrm{C}_{11 E / Z} \mathbf{H}\right), 7.21(\mathrm{~d}$, $\left.J=7.1 \mathrm{~Hz}, 2 \mathrm{H}, 2 \times \mathrm{C}_{9 E / Z} \mathbf{H}\right), 7.12(\mathrm{~d}, J=7.2 \mathrm{~Hz}, 2 \mathrm{H}$, $\left.2 \times \mathrm{C}_{9 E / Z} \mathbf{H}\right), 4.84\left(\mathrm{~d}, J=14.8 \mathrm{~Hz}, 1 \mathrm{H}, \mathrm{C}_{7 E / Z} \mathbf{H}\right), 4.74$ $\left(\mathrm{s}, 1 \mathrm{H}, \mathrm{C}_{2} \mathbf{H}\right), 4.54-4.42\left(\mathrm{~m}, 3 \mathrm{H}, 3 \times \mathrm{C}_{7 E / Z} \mathbf{H}\right), 2.02(\mathrm{~s}$, $\left.3 \mathrm{H}, \mathrm{C}_{12} \mathbf{H}_{3}\right)$.

$\delta 170.5\left(\mathbf{C}_{\mathbf{1}}\right), 137.1\left(\mathbf{C}_{\mathrm{Ar}}\right), 136.8\left(\mathbf{C}_{\mathbf{3}}\right), 136.4\left(\mathbf{C}_{\mathrm{Ar}}\right)$, $129.1\left(2 \times \mathbf{C}_{\mathrm{Ar}}\right), 128.8\left(2 \times \mathbf{C}_{\mathrm{Ar}}\right), 128.7\left(4 \times \mathbf{C}_{\mathrm{Ar}}\right)$, $128.3\left(2 \times \mathbf{C}_{\mathbf{9 E} / Z}\right), 128.1\left(\mathbf{C}_{\mathrm{Ar}}\right), 127.9\left(\mathbf{C}_{\mathrm{Ar}}\right), 127.5$ $\left(\mathbf{C}_{\mathbf{A r}}\right), 126.4\left(2 \times \mathbf{C}_{\mathbf{9 E / Z}}\right), 53.0\left(\mathbf{C}_{2}\right), 50.2\left(\mathbf{C}_{7 E / Z}\right), 49.1$ $\left(\mathbf{C}_{7 E / Z}\right), 14.8\left(\mathbf{C}_{12}\right)$.

3027 (w), 2914 (w), 1640 (s), 1494 (m), 1452 (m), 1418 (s), 1359 (m), 1193 (m), 1169 (m), 1077 (m), 1029 (m), 948 (m), 732 (m), 695 (s), 509 (m). 
HRMS (DART-TOF+) $m / z$ :

TLC (33\% EtOAc in hexanes), Rf:
$[\mathrm{M}+\mathrm{H}]^{+}$Calcd for $\mathrm{C}_{23} \mathrm{H}_{24} \mathrm{NOS}^{+} 362.1573$;

Found 362.1566.

$0.70(\mathrm{UV}, \mathrm{CAM})$. 


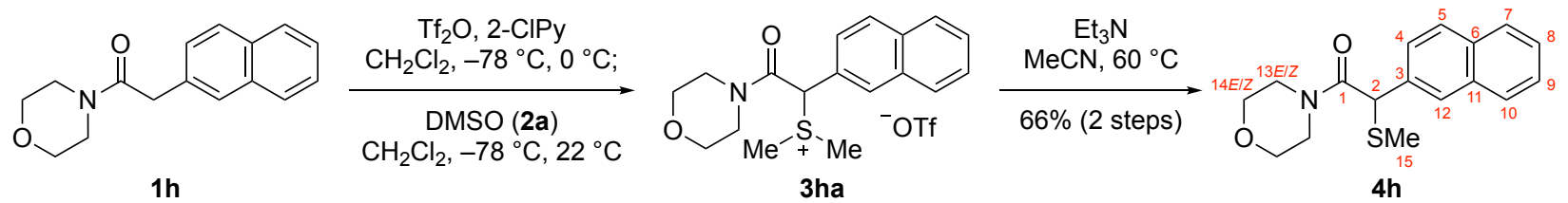

a-SMe-Morpholine Amide 4h:

The $\alpha$-SMe-morpholine amide $\mathbf{4 h}$ was prepared via Method A using azeotropically dried morpholine amide $\mathbf{1 h}(128 \mathrm{mg}, 500 \mu \mathrm{mol}, 1$ equiv) and dimethyl sulfoxide (2a, $42.6 \mu \mathrm{L}, 600 \mu \mathrm{mol}$, 1.20 equiv). The brown oily crude material was purified via flash column chromatography on silica gel (eluent: 33\% EtOAc in hexanes) to yield $\alpha$-SMe-morpholine amide $4 \mathbf{h}(99.5 \mathrm{mg}, 66 \%)$ as a solid. Structural assignments were made using additional information from gCOSY, gHSQC and gHMBC experiments.

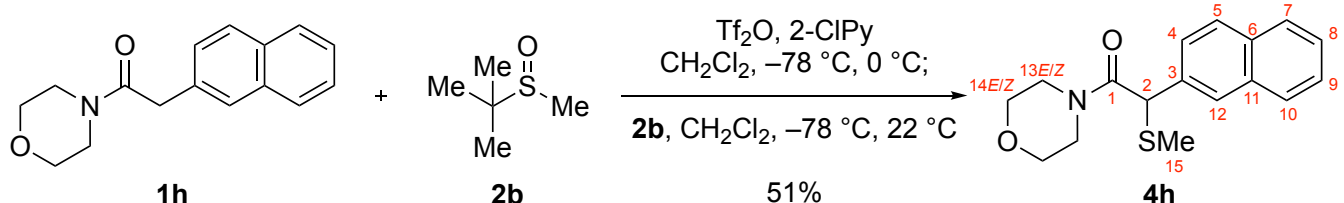

a-SMe-Morpholine Amide 4h:

The $\alpha$-SMe-morpholine amide $\mathbf{4 h}$ was prepared via Method B using azeotropically dried morpholine amide $\mathbf{1 h}(128 \mathrm{mg}, 500 \mu \mathrm{mol}, 1$ equiv) and tert-butyl methyl sulfoxide $(\mathbf{2 b}, 69.8 \mu \mathrm{L}$, $600 \mu \mathrm{mol}, 1.20$ equiv). The light yellow oily crude material was purified via flash column chromatography on silica gel (eluent: $33 \%$ EtOAc in hexanes) to yield $\alpha$-SMe-morpholine amide $\mathbf{4 h}(77.4 \mathrm{mg}, 51 \%)$ as a solid. The analytic data is fully consistent with that of compound $\mathbf{4 h}$ from Method A.

${ }^{1} \mathrm{H}$ NMR $\left(400 \mathrm{MHz}, \mathrm{CDCl}_{3}, 25^{\circ} \mathrm{C}\right): \quad \delta 7.87-7.80\left(\mathrm{~m}, 3 \mathrm{H}, \mathrm{C}_{5} \mathbf{H}, \mathrm{C}_{7} \mathbf{H}, \mathrm{C}_{10} \mathbf{H}\right), 7.76(\mathrm{~s}, 1 \mathrm{H}$, $\left.\mathrm{C}_{12} \mathbf{H}\right), 7.58\left(\mathrm{dd}, J=8.6,1.8 \mathrm{~Hz}, 1 \mathrm{H}, \mathrm{C}_{4} \mathbf{H}\right), 7.51-7.47$ $\left(\mathrm{m}, 2 \mathrm{H}, \mathrm{C}_{8} \mathbf{H}, \mathrm{C}_{9} \mathbf{H}\right), 4.88\left(\mathrm{~s}, 1 \mathrm{H}, \mathrm{C}_{2} \mathbf{H}\right), 3.74-3.48(\mathrm{~m}$, $\left.6 \mathrm{H}, 3 \times \mathrm{C}_{13 E / Z} \mathbf{H}, 3 \times \mathrm{C}_{14 E / Z} \mathbf{H}\right), 3.42-3.36(\mathrm{~m}, 1 \mathrm{H}$, $\left.\mathrm{C}_{13 E / Z} \mathbf{H}\right), 3.28-3.23\left(\mathrm{~m}, 1 \mathrm{H}, \mathrm{C}_{14 E / Z} \mathbf{H}\right), 2.02(\mathrm{~s}, 3 \mathrm{H}$, $\left.\mathrm{C}_{15} \mathbf{H}_{3}\right)$.

${ }^{13} \mathrm{C} \mathrm{NMR}\left(100 \mathrm{MHz}, \mathrm{CDCl}_{3}, 25^{\circ} \mathrm{C}\right): \quad \delta 168.3\left(\mathbf{C}_{\mathbf{1}}\right), 134.0\left(\mathbf{C}_{\mathbf{3}}\right), 133.2\left(\mathbf{C}_{11}\right), 133.0\left(\mathbf{C}_{\mathbf{6}}\right)$, $129.1\left(\mathbf{C}_{\mathrm{Ar}}\right), 128.0\left(\mathbf{C}_{\mathbf{A r}}\right), 127.8\left(\mathbf{C}_{\mathbf{A r}}\right), 127.2\left(\mathbf{C}_{12}\right)$, $126.6\left(\mathbf{C}_{\text {Ar }}\right), 126.5\left(\mathbf{C}_{\text {Ar }}\right), 126.0\left(\mathbf{C}_{4}\right), 66.9\left(\mathbf{C}_{14 E / Z}\right)$, $66.4\left(\mathbf{C}_{14 E / Z}\right), 53.7\left(\mathbf{C}_{2}\right), 46.6\left(\mathbf{C}_{13 E / Z}\right), 42.8\left(\mathbf{C}_{13 E / Z}\right)$, $14.6\left(\mathbf{C}_{15}\right)$.

FTIR (thin film) $\mathrm{cm}^{-1}$ : $2970(\mathrm{w}), 2918(\mathrm{w}), 2851(\mathrm{w}), 1640(\mathrm{~s}), 1422(\mathrm{~m})$, 1237 (m), 1107 (s), 1029 (m), 802 (s), 749 (m), 662 (m), $581(\mathrm{~m}), 477(\mathrm{~s})$.

HRMS (DART-TOF+) $m / z$ :

$[\mathrm{M}+\mathrm{H}]^{+}$Calcd for $\mathrm{C}_{17} \mathrm{H}_{20} \mathrm{NO}_{2} \mathrm{~S}^{+} 302.1209$;

Found 302.1201.

TLC (67\% EtOAc in hexanes), Rf: 0.36 (UV, CAM). 
M.p.:

$98-100{ }^{\circ} \mathrm{C}\left(\mathrm{CH}_{2} \mathrm{Cl}_{2}\right)$. 
<smiles>O=C(Cc1cccs1)N1CCOCC1</smiles>

$1 \mathrm{i}$

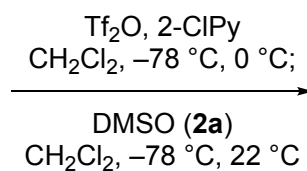

$\mathrm{CH}_{2} \mathrm{Cl}_{2},-78^{\circ} \mathrm{C}, 22^{\circ} \mathrm{C}$
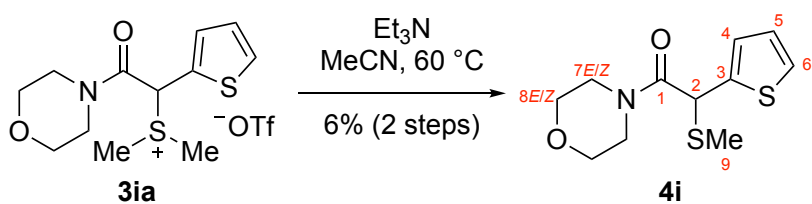

\section{$\alpha$-SMe-Morpholine Amide 4i:}

The $\alpha$-SMe-morpholine amide $4 \mathbf{i}$ was prepared via Method A using azeotropically dried morpholine amide 1i (106 mg, $500 \mu \mathrm{mol}, 1$ equiv) and dimethyl sulfoxide (2a, $42.6 \mu \mathrm{L}, 600 \mu \mathrm{mol}$, 1.20 equiv). The brown oily crude material was purified via flash column chromatography on silica gel (eluent: 33\% EtOAc in hexanes) to yield $\alpha$-SMe-morpholine amide $4 \mathbf{i}(7.7 \mathrm{mg}, 6 \%)$ as a solid. Structural assignments were made using additional information from gCOSY, gHSQC and gHMBC experiments.
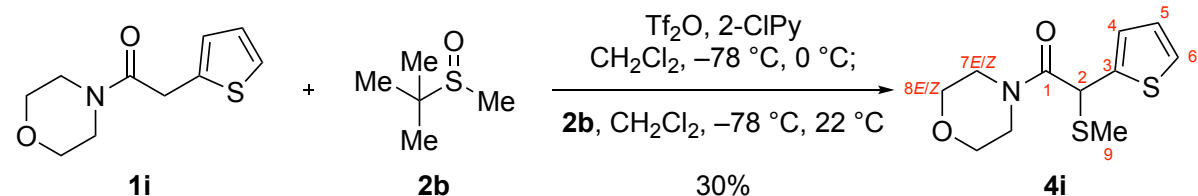

\section{$\alpha$-SMe-Morpholine Amide 4i:}

The $\alpha$-SMe-morpholine amide $4 \mathbf{i}$ was prepared via Method B using azeotropically dried morpholine amide 1i (106 mg, $500 \mu \mathrm{mol}, 1$ equiv) and tert-butyl methyl sulfoxide $(\mathbf{2 b}, 69.8 \mu \mathrm{L}$, $600 \mu \mathrm{mol}, 1.20$ equiv). The light yellow oily crude material was purified via flash column chromatography on silica gel (eluent: $33 \%$ EtOAc in hexanes) to yield $\alpha$-SMe-morpholine amide $4 \mathbf{i}(38.3 \mathrm{mg}, 30 \%)$ as a solid. The analytic data is fully consistent with that of compound $\mathbf{4 i}$ from Method A.

${ }^{1} \mathrm{H}$ NMR (400 MHz, $\left.\mathrm{CDCl}_{3}, 25^{\circ} \mathrm{C}\right)$ :

${ }^{13} \mathrm{C}$ NMR $\left(100 \mathrm{MHz}, \mathrm{CDCl}_{3}, 25{ }^{\circ} \mathrm{C}\right)$ :

FTIR (thin film) $\mathrm{cm}^{-1}$ :

HRMS (DART-TOF+) $m / z$ :

TLC (67\% EtOAc in hexanes), Rf:

M.p.: $\delta 7.30\left(\mathrm{dd}, J=5.2,1.3 \mathrm{~Hz}, 1 \mathrm{H}, \mathrm{C}_{6} \mathbf{H}\right), 7.04(\mathrm{dd}, J=$ 3.6, $\left.1.0 \mathrm{~Hz}, 1 \mathrm{H}, \mathrm{C}_{4} \mathbf{H}\right), 6.95(\mathrm{dd}, J=5.2,3.5 \mathrm{~Hz}, 1 \mathrm{H}$, $\left.\mathrm{C}_{5} \mathbf{H}\right), 5.02\left(\mathrm{~s}, 1 \mathrm{H}, \mathrm{C}_{2} \mathbf{H}\right), 3.72-3.47(\mathrm{~m}, 8 \mathrm{H}, 4 \times$ $\left.\mathrm{C}_{7 E / Z} \mathbf{H}, 4 \times \mathrm{C}_{8 E / Z} \mathbf{H}\right), 2.10\left(\mathrm{~s}, 3 \mathrm{H}, \mathrm{C}_{9} \mathbf{H}_{3}\right)$.

$\delta 167.5\left(\mathbf{C}_{\mathbf{1}}\right), 139.6\left(\mathbf{C}_{\mathbf{3}}\right), 126.7\left(\mathbf{C}_{\mathbf{4}}\right), 126.7\left(\mathbf{C}_{\mathbf{6}}\right)$, $126.4\left(\mathbf{C}_{5}\right), 66.9\left(\mathbf{C}_{8 E / Z}\right), 66.5\left(\mathbf{C}_{8 E / Z}\right), 46.9\left(\mathbf{C}_{7 E / Z}\right)$, $46.3\left(\mathbf{C}_{2}\right), 42.9\left(\mathbf{C}_{7 E / Z}\right), 14.4\left(\mathbf{C}_{9}\right)$.

$2957(\mathrm{w}), 2907(\mathrm{w}), 2857(\mathrm{w}), 1621$ (s), 1433 (s), 1219 (s), 1111 (s), 1035 (m), 853 (m), 800 (m), 703 (s), $661(\mathrm{~m}), 628(\mathrm{~m}), 592(\mathrm{~m}), 571(\mathrm{~m}), 501(\mathrm{~m})$.

$[\mathrm{M}+\mathrm{H}]^{+}$Calcd for $\mathrm{C}_{11} \mathrm{H}_{16} \mathrm{NO}_{2} \mathrm{~S}_{2}{ }^{+}$258.0617; Found 258.0618.

0.53 (UV, CAM). $60-62{ }^{\circ} \mathrm{C}\left(\mathrm{CH}_{2} \mathrm{Cl}_{2}\right)$. 


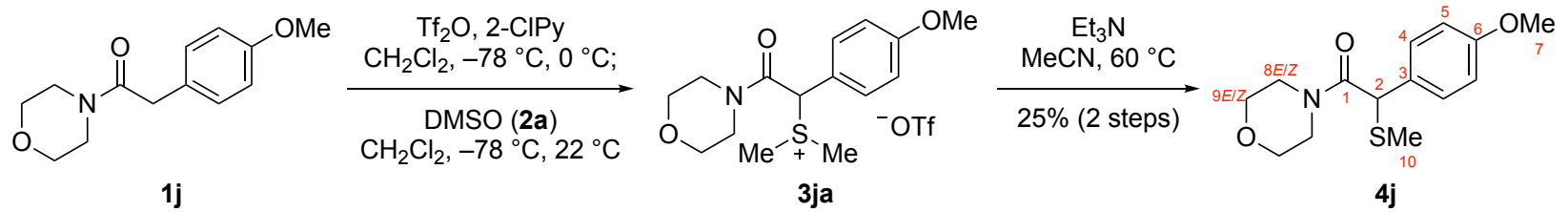

a-SMe-Morpholine Amide 4j:

The $\alpha$-SMe-morpholine amide $\mathbf{4 j}$ was prepared via Method A using azeotropically dried morpholine amide $\mathbf{1 j}$ (118 mg, $500 \mu \mathrm{mol}, 1$ equiv) and dimethyl sulfoxide (2a, $42.6 \mu \mathrm{L}, 600 \mu \mathrm{mol}$, 1.20 equiv). The brown oily crude material was purified via flash column chromatography on silica gel (eluent: $50 \%$ EtOAc in hexanes) to yield $\alpha$-SMe-morpholine amide $\mathbf{4 j}(34.5 \mathrm{mg}, 25 \%)$ as an oil. Structural assignments were made using additional information from gCOSY, gHSQC and gHMBC experiments.

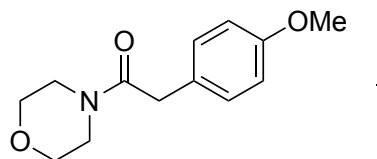

$1 \mathrm{j}$

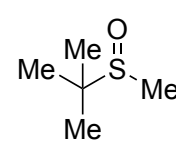

2b

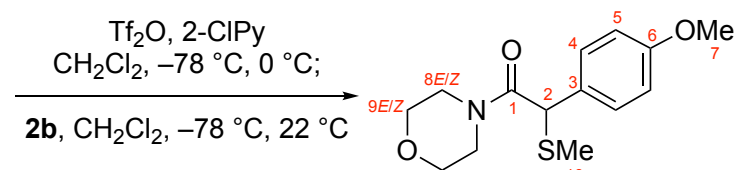

$23 \%$

4j

$\alpha$-SMe-Morpholine Amide 4j:

The $\alpha$-SMe-morpholine amide $\mathbf{4 j}$ was prepared via Method B using azeotropically dried morpholine amide $\mathbf{1 j}$ (118 mg, $500 \mu \mathrm{mol}, 1$ equiv) and tert-butyl methyl sulfoxide $(\mathbf{2 b}, 69.8 \mu \mathrm{L}$, $600 \mu \mathrm{mol}, 1.20$ equiv). The light yellow oily crude material was purified via flash column chromatography on silica gel (eluent: $50 \%$ EtOAc in hexanes) to yield $\alpha$-SMe-morpholine amide $\mathbf{4 j}(32.3 \mathrm{mg}, 23 \%)$ as an oil. The analytic data is fully consistent with that of compound $\mathbf{4 j}$ from Method A.

${ }^{1} \mathrm{H}$ NMR $\left(400 \mathrm{MHz}, \mathrm{CDCl}_{3}, 25^{\circ} \mathrm{C}\right)$ :

${ }^{13} \mathrm{C} \mathrm{NMR}\left(100 \mathrm{MHz}, \mathrm{CDCl}_{3}, 25^{\circ} \mathrm{C}\right)$ :

FTIR (thin film) $\mathrm{cm}^{-1}$ :

HRMS (DART-TOF+) $m / z$ :

TLC (67\% EtOAc in hexanes), Rf: $\delta 7.29\left(\mathrm{~d}, J=8.4 \mathrm{~Hz}, 2 \mathrm{H}, 2 \times \mathrm{C}_{4} \mathbf{H}\right), 6.87(\mathrm{~d}, J=$ $\left.8.2 \mathrm{~Hz}, 2 \mathrm{H}, 2 \times \mathrm{C}_{5} \mathbf{H}\right), 4.67\left(\mathrm{~s}, 1 \mathrm{H}, \mathrm{C}_{2} \mathbf{H}\right), 3.79(\mathrm{~s}, 3 \mathrm{H}$, $\left.\mathrm{C}_{7} \mathbf{H}_{3}\right), 3.71-3.43\left(\mathrm{~m}, 6 \mathrm{H}, 3 \times \mathrm{C}_{8 E / Z} \mathbf{H}, 3 \times \mathrm{C}_{9 E / Z} \mathbf{H}\right)$, 3.38-3.33 (m, 1H, $\left.\mathrm{C}_{8 E / Z} \mathbf{H}\right), 3.30-3.24(\mathrm{~m}, 1 \mathrm{H}$, $\left.\mathrm{C}_{9 E / Z} \mathbf{H}\right), 1.99\left(\mathrm{~s}, 3 \mathrm{H}, \mathrm{C}_{10} \mathbf{H}_{3}\right)$.

$\delta 168.5\left(\mathbf{C}_{\mathbf{1}}\right), 159.3\left(\mathbf{C}_{\mathbf{6}}\right), 129.5\left(2 \times \mathbf{C}_{4}\right), 128.6\left(\mathbf{C}_{\mathbf{3}}\right)$, $114.3\left(2 \times \mathbf{C}_{5}\right), 66.9\left(\mathbf{C}_{\mathbf{9 E} / Z}\right), 66.4\left(\mathbf{C}_{9 E / Z}\right), 55.4\left(\mathbf{C}_{7}\right)$, $52.8\left(\mathbf{C}_{2}\right), 46.6\left(\mathbf{C}_{8 E / Z}\right), 42.8\left(\mathbf{C}_{8 E / Z}\right), 14.6\left(\mathbf{C}_{10}\right)$.

2962 (w), $2915(\mathrm{w}), 2853(\mathrm{w}), 1634(\mathrm{~s}), 1607$ (m), $1509(\mathrm{~s}), 1429(\mathrm{~s}), 1247(\mathrm{~s}), 1220(\mathrm{~s}), 1174(\mathrm{~s}), 1110$ (s), $1067(\mathrm{w}), 1030(\mathrm{~s}), 958(\mathrm{w}), 852(\mathrm{~m}), 835(\mathrm{~m})$, $791(\mathrm{~m}), 760(\mathrm{~m}), 572(\mathrm{~m}), 528(\mathrm{~m})$.

$[\mathrm{M}+\mathrm{H}]^{+}$Calcd for $\mathrm{C}_{14} \mathrm{H}_{20} \mathrm{NO}_{3} \mathrm{~S}^{+} 282.1158$;

Found 282.1152.

0.38 (UV, CAM). 


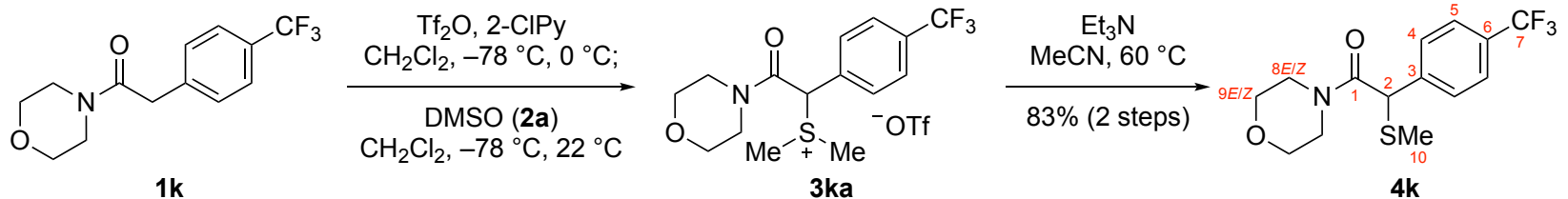

$\alpha$-SMe-Morpholine Amide 4k:

The $\alpha$-SMe-morpholine amide $\mathbf{4 k}$ was prepared via Method A using azeotropically dried morpholine amide $\mathbf{1 k}(137 \mathrm{mg}, 500 \mu \mathrm{mol}, 1$ equiv) and dimethyl sulfoxide (2a, $42.6 \mu \mathrm{L}, 600 \mu \mathrm{mol}$, 1.20 equiv). The brown oily crude material was purified via flash column chromatography on silica gel (eluent: 33\% EtOAc in hexanes) to yield $\alpha$-SMe-morpholine amide $4 \mathbf{k}(133 \mathrm{mg}, 83 \%)$ as a solid. Structural assignments were made using additional information from gCOSY, gHSQC and gHMBC experiments.

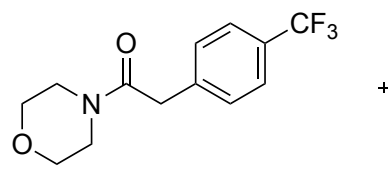

$1 \mathrm{k}$

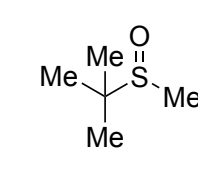

$2 \mathbf{b}$

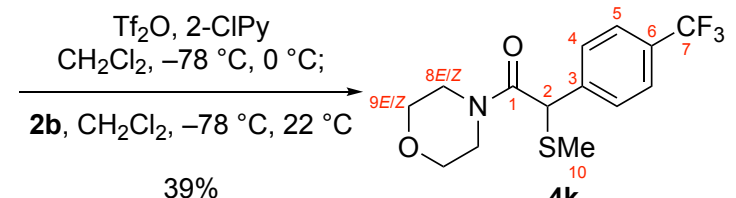

$39 \%$

\section{-SMe-Morpholine Amide 4k:}

The $\alpha$-SMe-morpholine amide $\mathbf{4 k}$ was prepared via Method B using azeotropically dried morpholine amide $\mathbf{1 k}(137 \mathrm{mg}, 500 \mu \mathrm{mol}, 1$ equiv) and tert-butyl methyl sulfoxide $(\mathbf{2 b}, 69.8 \mu \mathrm{L}$, $600 \mu \mathrm{mol}, 1.20$ equiv). The light yellow oily crude material was purified via flash column chromatography on silica gel (eluent: $33 \%$ EtOAc in hexanes) to yield $\alpha$-SMe-morpholine amide $\mathbf{4 k}(61.8 \mathrm{mg}, 39 \%)$ as a solid. The analytic data is fully consistent with that of compound $\mathbf{4 k}$ from Method A.

${ }^{1} \mathrm{H}$ NMR (400 MHz, $\left.\mathrm{CDCl}_{3}, 25^{\circ} \mathrm{C}\right)$ :

${ }^{13} \mathrm{C}$ NMR (100 MHz, $\left.\mathrm{CDCl}_{3}, 25^{\circ} \mathrm{C}\right)$ :

${ }^{19} \mathrm{~F}$ NMR $\left(376 \mathrm{MHz}, \mathrm{CDCl}_{3}, 25^{\circ} \mathrm{C}\right)$ :

FTIR (thin film) $\mathrm{cm}^{-1}$ :

HRMS (DART-TOF+) $m / z$ :

TLC (67\% EtOAc in hexanes), Rf: $\delta 7.62\left(\mathrm{~d}, J=8.1 \mathrm{~Hz}, 2 \mathrm{H}, 2 \times \mathrm{C}_{5} \mathbf{H}\right), 7.54(\mathrm{~d}, J=$ $\left.8.1 \mathrm{~Hz}, 2 \mathrm{H}, 2 \times \mathrm{C}_{4} \mathbf{H}\right), 4.73\left(\mathrm{~s}, 1 \mathrm{H}, \mathrm{C}_{2} \mathbf{H}\right), 3.70-3.50$ $\left(\mathrm{m}, 6 \mathrm{H}, 3 \times \mathrm{C}_{8 E / Z} \mathbf{H}, 3 \times \mathrm{C}_{9 E / Z} \mathbf{H}\right), 3.45-3.33(\mathrm{~m}, 2 \mathrm{H}$, $\left.\mathrm{C}_{8 E / Z} \mathbf{H}, \mathrm{C}_{9 E / Z} \mathbf{H}\right), 2.01\left(\mathrm{~s}, 3 \mathrm{H}, \mathrm{C}_{10} \mathbf{H}_{3}\right)$.

$\delta 167.6\left(\mathbf{C}_{1}\right), 140.9\left(\mathbf{C}_{3}\right), 130.3\left(\mathrm{q}, J=32.5 \mathrm{~Hz}, \mathbf{C}_{\mathbf{6}}\right)$, $129.0\left(2 \times \mathbf{C}_{4}\right), 125.8\left(\mathrm{q}, J=3.7 \mathrm{~Hz}, 2 \times \mathbf{C}_{5}\right), 124.0$ $\left(\mathrm{q}, J=272 \mathrm{~Hz}, \mathbf{C}_{7}\right), 66.8\left(\mathbf{C}_{\mathbf{9 E / Z}}\right), 66.5\left(\mathbf{C}_{\mathbf{9 E / Z}}\right), 52.2$ $\left(\mathbf{C}_{2}\right), 46.7\left(\mathbf{C}_{8 E / Z}\right), 42.9\left(\mathbf{C}_{8 E / Z}\right), 14.7\left(\mathbf{C}_{10}\right)$.

$\delta-63.6$.

2917 (w), 2863 (w), 1633 (s), 1615 (m), 1433 (m), 1323 (s), $1224(\mathrm{~m}), 1162$ (s), 1107 (s), 1065 (s), 1033 (m), $1004(\mathrm{~m}), 851(\mathrm{~m}), 782(\mathrm{~m}), 705(\mathrm{~m}), 603(\mathrm{~m})$, $587(\mathrm{~m})$.

$[\mathrm{M}+\mathrm{H}]^{+}$Calcd for $\mathrm{C}_{14} \mathrm{H}_{17} \mathrm{~F}_{3} \mathrm{NO}_{2} \mathrm{~S}^{+} 320.0927$;

Found 320.0923.

0.41 (UV, CAM). 
M.p.:

76-78 ${ }^{\circ} \mathrm{C}\left(\mathrm{CH}_{2} \mathrm{Cl}_{2}\right)$. 


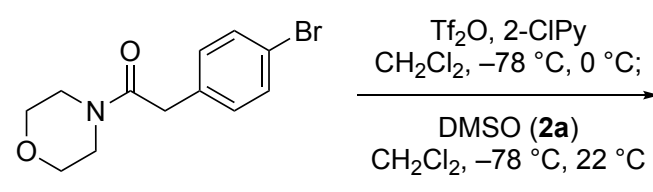

11

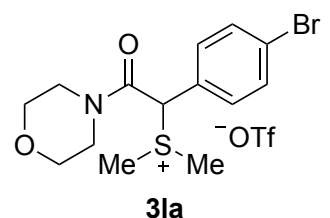

3la

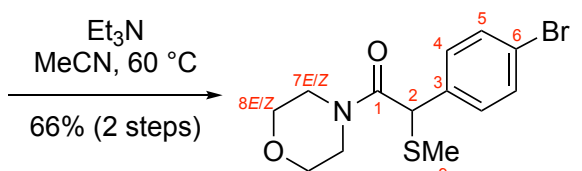

41

a-SMe-Morpholine Amide 4l:

The $\alpha$-SMe-morpholine amide $\mathbf{4 l}$ was prepared via Method A using azeotropically dried morpholine amide 11 (142 mg, $500 \mu \mathrm{mol}, 1$ equiv) and dimethyl sulfoxide (2a, $42.6 \mu \mathrm{L}, 600 \mu \mathrm{mol}$, 1.20 equiv). The brown oily crude material was purified via flash column chromatography on silica gel (eluent: 33\% EtOAc in hexanes) to yield $\alpha$-SMe-morpholine amide $4 \mathbf{l}(109 \mathrm{mg}, 66 \%)$ as an oil. Structural assignments were made using additional information from gCOSY, gHSQC and gHMBC experiments.

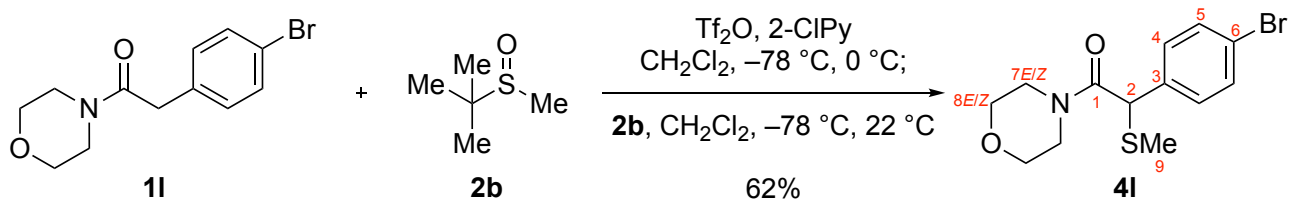

\section{$\alpha$-SMe-Morpholine Amide 4l:}

The $\alpha$-SMe-morpholine amide $4 \mathbf{l}$ was prepared via Method B using azeotropically dried morpholine amide 11 (142 mg, $500 \mu \mathrm{mol}, 1$ equiv) and tert-butyl methyl sulfoxide $(\mathbf{2 b}, 69.8 \mu \mathrm{L}$, $600 \mu \mathrm{mol}, 1.20$ equiv). The light yellow oily crude material was purified via flash column chromatography on silica gel (eluent: $33 \%$ EtOAc in hexanes) to yield $\alpha$-SMe-morpholine amide $41(102 \mathrm{mg}, 62 \%)$ as an oil. The analytic data is fully consistent with that of compound $4 \mathbf{l}$ from Method A.

${ }^{1} \mathrm{H}$ NMR $\left(400 \mathrm{MHz}, \mathrm{CDCl}_{3}, 25^{\circ} \mathrm{C}\right)$ :

${ }^{13} \mathrm{C}$ NMR $\left(100 \mathrm{MHz}, \mathrm{CDCl}_{3}, 25^{\circ} \mathrm{C}\right)$ :

FTIR (thin film) $\mathrm{cm}^{-1}$ :

HRMS (DART-TOF+) $m / z$ :

TLC (67\% EtOAc in hexanes), Rf: $\delta 7.48\left(\mathrm{~d}, J=8.3 \mathrm{~Hz}, 2 \mathrm{H}, 2 \times \mathrm{C}_{5} \mathbf{H}\right), 7.28(\mathrm{~d}, J=$ $\left.8.3 \mathrm{~Hz}, 2 \mathrm{H}, 2 \times \mathrm{C}_{4} \mathbf{H}\right), 4.65\left(\mathrm{~s}, 1 \mathrm{H}, \mathrm{C}_{2} \mathbf{H}\right), 3.68-3.45$ $\left(\mathrm{m}, 6 \mathrm{H}, 3 \times \mathrm{C}_{7 E / Z} \mathbf{H}, 3 \times \mathrm{C}_{8 E / Z} \mathbf{H}\right), 3.40-3.32(\mathrm{~m}, 2 \mathrm{H}$, $\left.\mathrm{C}_{7 E / Z} \mathbf{H}, \mathrm{C}_{8 E / Z} \mathbf{H}\right), 2.00\left(\mathrm{~s}, 3 \mathrm{H}, \mathrm{C}_{9} \mathbf{H}_{3}\right)$.

$\delta 167.8\left(\mathbf{C}_{\mathbf{1}}\right), 135.8\left(\mathbf{C}_{\mathbf{3}}\right), 132.0\left(2 \times \mathbf{C}_{\mathbf{5}}\right), 130.2(2 \times$ $\left.\mathbf{C}_{4}\right), 122.1\left(\mathbf{C}_{6}\right), 66.8\left(\mathbf{C}_{8 E / Z}\right), 66.5\left(\mathbf{C}_{8 E / Z}\right), 52.3\left(\mathbf{C}_{2}\right)$, $46.6\left(\mathbf{C}_{7 E / Z}\right), 42.8\left(\mathbf{C}_{7 E / Z}\right), 14.7\left(\mathbf{C}_{9}\right)$.

2963 (w), 2915 (w), 2853 (w), 1633 (s), 1486 (m), $1427(\mathrm{~s}), 1273(\mathrm{~m}), 1216(\mathrm{~m}), 1111(\mathrm{~s}), 1069(\mathrm{~m})$, $1033(\mathrm{~m}), 1009(\mathrm{~m}), 852(\mathrm{~m}), 768(\mathrm{~m}), 573(\mathrm{~m}), 507$ (m).

$[\mathrm{M}+\mathrm{H}]^{+}$Calcd for $\mathrm{C}_{13} \mathrm{H}_{17} \mathrm{BrNO}_{2} \mathrm{~S}^{+} 330.0158$; Found 330.0155.

0.46 (UV, CAM). 


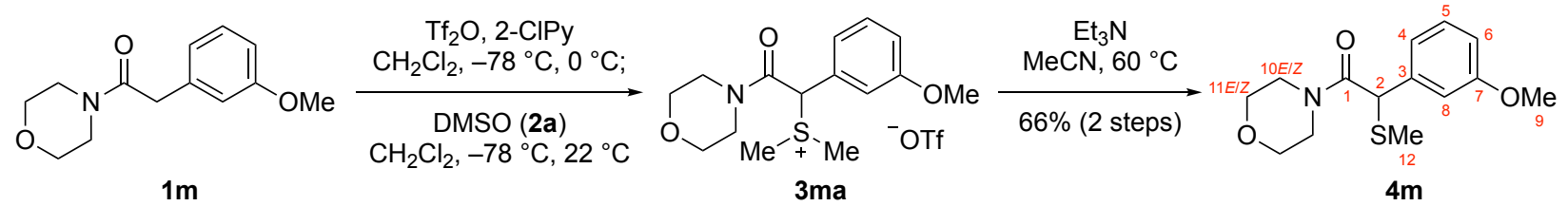

Q-SMe-Morpholine Amide 4m:

The $\alpha$-SMe-morpholine amide $\mathbf{4 m}$ was prepared via Method A using azeotropically dried morpholine amide $\mathbf{1 m}$ (118 mg, $500 \mu \mathrm{mol}, 1$ equiv) and dimethyl sulfoxide $(\mathbf{2 a}, 42.6 \mu \mathrm{L}, 600 \mu \mathrm{mol}$, 1.20 equiv). The brown oily crude material was purified via flash column chromatography on silica gel (eluent: $33 \%$ EtOAc in hexanes) to yield $\alpha$-SMe-morpholine amide $\mathbf{4 m}(92.6 \mathrm{mg}, 66 \%)$ as an oil. Structural assignments were made using additional information from gCOSY, gHSQC and gHMBC experiments.
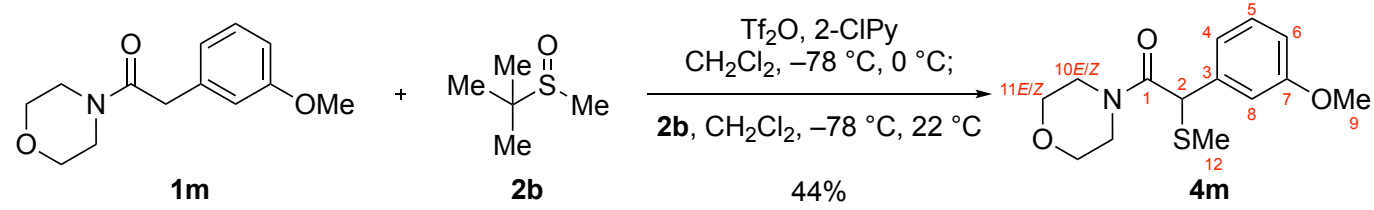

\section{a-SMe-Morpholine Amide 4m:}

The $\alpha$-SMe-morpholine amide $\mathbf{4 m}$ was prepared via Method B using azeotropically dried morpholine amide $\mathbf{1 m}(118 \mathrm{mg}, 500 \mu \mathrm{mol}, 1$ equiv) and tert-butyl methyl sulfoxide $(\mathbf{2 b}, 69.8 \mu \mathrm{L}$, $600 \mu \mathrm{mol}, 1.20$ equiv). The light yellow oily crude material was purified via flash column chromatography on silica gel (eluent: $33 \%$ EtOAc in hexanes) to yield $\alpha$-SMe-morpholine amide $\mathbf{4 m}(61.6 \mathrm{mg}, 44 \%)$ as an oil. The analytic data is fully consistent with that of compound $\mathbf{4 m}$ from Method A.

${ }^{1} \mathrm{H}$ NMR $\left(400 \mathrm{MHz}, \mathrm{CDCl}_{3}, 25^{\circ} \mathrm{C}\right)$ :

${ }^{13} \mathrm{C}$ NMR $\left(100 \mathrm{MHz}, \mathrm{CDCl}_{3}, 25{ }^{\circ} \mathrm{C}\right)$ :

FTIR (thin film) $\mathrm{cm}^{-1}$ :

HRMS (DART-TOF+) $m / z$ :

TLC (67\% EtOAc in hexanes), Rf: $\delta 7.24\left(\mathrm{t}, J=8.3 \mathrm{~Hz}, 1 \mathrm{H}, \mathrm{C}_{5} \mathbf{H}\right), 6.95-6.90(\mathrm{~m}, 2 \mathrm{H}$, $\left.\mathrm{C}_{4} \mathbf{H}, \mathrm{C}_{8} \mathbf{H}\right), 6.81\left(\mathrm{dd}, J=8.3,1.9 \mathrm{~Hz}, 1 \mathrm{H}, \mathrm{C}_{6} \mathbf{H}\right), 4.68$ $\left(\mathrm{s}, 1 \mathrm{H}, \mathrm{C}_{2} \mathbf{H}\right), 3.79\left(\mathrm{~s}, 3 \mathrm{H}, \mathrm{C}_{9} \mathbf{H}_{3}\right), 3.71-3.40(\mathrm{~m}, 6 \mathrm{H}$, $\left.3 \times \mathrm{C}_{10 E / Z} \mathbf{H}, 3 \times \mathrm{C}_{11 E / Z} \mathbf{H}\right), 3.37-3.32(\mathrm{~m}, 1 \mathrm{H}$, $\left.\mathrm{C}_{10 E / Z} \mathbf{H}\right), 3.30-3.24\left(\mathrm{~m}, 1 \mathrm{H}, \mathrm{C}_{11 E / Z} \mathbf{H}\right), 2.01(\mathrm{~s}, 3 \mathrm{H}$, $\left.\mathrm{C}_{12} \mathbf{H}_{3}\right)$.

$\delta 168.2\left(\mathbf{C}_{\mathbf{1}}\right), 160.1\left(\mathbf{C}_{7}\right), 138.2\left(\mathbf{C}_{\mathbf{3}}\right), 129.8\left(\mathbf{C}_{\mathbf{5}}\right)$, $120.6\left(\mathbf{C}_{4}\right), 113.7\left(\mathbf{C}_{8}\right), 113.6\left(\mathbf{C}_{6}\right), 66.8\left(\mathbf{C}_{11 E / Z}\right), 66.4$ $\left(\mathbf{C}_{11 E / Z}\right), 55.4\left(\mathbf{C}_{9}\right), 53.6\left(\mathbf{C}_{2}\right), 46.6\left(\mathbf{C}_{10 E / Z}\right), 42.8$ (C10E/Z), $14.7\left(\mathbf{C}_{12}\right)$.

$2962(\mathrm{w}), 2916(\mathrm{w}), 2853(\mathrm{w}), 1636(\mathrm{~s}), 1596(\mathrm{~m})$, 1426 (s), 1252 (s), $1214(\mathrm{~m}), 1111(\mathrm{~s}), 1033$ (s), 959 (w), $849(\mathrm{w}), 778(\mathrm{~m}), 694(\mathrm{~m}), 573(\mathrm{~m})$.

$[\mathrm{M}+\mathrm{H}]^{+}$Calcd for $\mathrm{C}_{14} \mathrm{H}_{20} \mathrm{NO}_{3} \mathrm{~S}^{+} 282.1158$;

Found 282.1156.

0.43 (UV, CAM). 

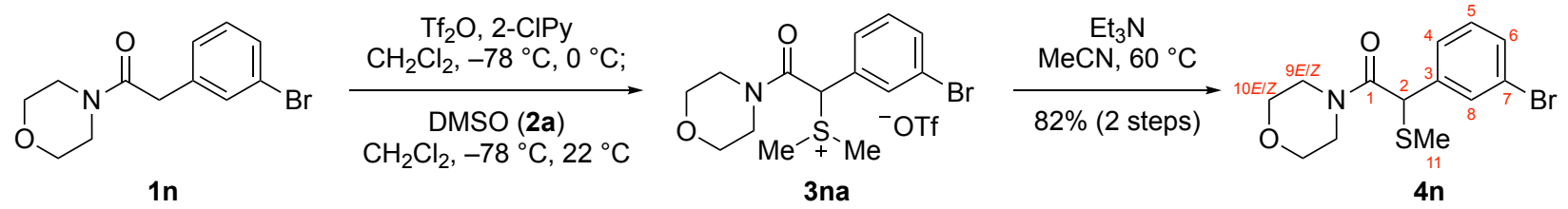

a-SMe-Morpholine Amide 4n:

The $\alpha$-SMe-morpholine amide $4 \mathbf{n}$ was prepared via Method A using azeotropically dried morpholine amide 1n (142 mg, $500 \mu \mathrm{mol}, 1$ equiv) and dimethyl sulfoxide (2a, $42.6 \mu \mathrm{L}, 600 \mu \mathrm{mol}$, 1.20 equiv). The brown oily crude material was purified via flash column chromatography on silica gel (eluent: 25\% EtOAc in hexanes) to yield $\alpha$-SMe-morpholine amide 4n (136 $\mathrm{mg}, 82 \%)$ as an oil. Structural assignments were made using additional information from gCOSY, gHSQC and gHMBC experiments.
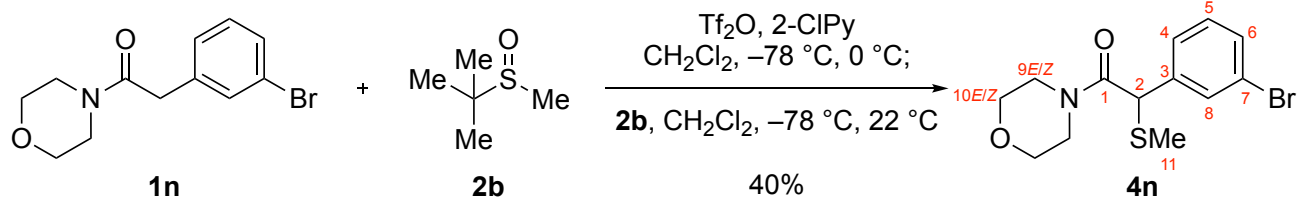

\section{a-SMe-Morpholine Amide 4n:}

The $\alpha$-SMe-morpholine amide $4 \mathbf{n}$ was prepared via Method B using azeotropically dried morpholine amide 1n (142 mg, $500 \mu \mathrm{mol}, 1$ equiv) and tert-butyl methyl sulfoxide $(\mathbf{2 b}, 69.8 \mu \mathrm{L}$, $600 \mu \mathrm{mol}, 1.20$ equiv). The light yellow oily crude material was purified via flash column chromatography on silica gel (eluent: $25 \%$ EtOAc in hexanes) to yield $\alpha$-SMe-morpholine amide 4n $(65.7 \mathrm{mg}, 40 \%)$ as an oil. The analytic data is fully consistent with that of compound $\mathbf{4 n}$ from Method A.

${ }^{1} \mathrm{H}$ NMR (400 MHz, $\left.\mathrm{CDCl}_{3}, 25^{\circ} \mathrm{C}\right)$ :

${ }^{13} \mathrm{C} \mathrm{NMR}\left(100 \mathrm{MHz}, \mathrm{CDCl}_{3}, 25^{\circ} \mathrm{C}\right)$ :

FTIR (thin film) $\mathrm{cm}^{-1}$ :

HRMS (DART-TOF+) $m / z$ :

TLC (67\% EtOAc in hexanes), Rf: $\delta 7.57\left(\mathrm{~s}, 1 \mathrm{H}, \mathrm{C}_{8} \mathbf{H}\right), 7.43\left(\mathrm{~d}, J=8.0 \mathrm{~Hz}, 1 \mathrm{H}, \mathrm{C}_{6} \mathbf{H}\right)$, $7.34\left(\mathrm{~d}, J=7.8 \mathrm{~Hz}, 1 \mathrm{H}, \mathrm{C}_{4} \mathbf{H}\right), 7.22(\mathrm{t}, J=7.8 \mathrm{~Hz}, 1 \mathrm{H}$, $\left.\mathrm{C}_{5} \mathbf{H}\right), 4.65\left(\mathrm{~s}, 1 \mathrm{H}, \mathrm{C}_{2} \mathbf{H}\right), 3.67-3.47(\mathrm{~m}, 6 \mathrm{H}, 3 \times$ $\left.\mathrm{C}_{9 E / Z} \mathbf{H}, 3 \times \mathrm{C}_{10 E / Z} \mathbf{H}\right), 3.42-3.33\left(\mathrm{~m}, 2 \mathrm{H}, \mathrm{C}_{9 E / Z} \mathbf{H}\right.$, $\left.\mathrm{C}_{10 E / Z} \mathbf{H}\right), 2.01\left(\mathrm{~s}, 3 \mathrm{H}, \mathrm{C}_{11} \mathbf{H}_{3}\right)$.

$\delta 167.7\left(\mathbf{C}_{\mathbf{1}}\right), 139.1\left(\mathbf{C}_{3}\right), 131.5\left(\mathbf{C}_{8}\right), 131.2\left(\mathbf{C}_{6}\right)$, $130.4\left(\mathbf{C}_{5}\right), 127.2\left(\mathbf{C}_{4}\right), 122.9\left(\mathbf{C}_{7}\right), 66.9\left(\mathbf{C}_{10 E / Z}\right), 66.5$ $\left(\mathbf{C}_{10 E / Z}\right), 52.3\left(\mathbf{C}_{2}\right), 46.7\left(\mathbf{C}_{9 E / Z}\right), 42.9\left(\mathbf{C}_{9 E / Z}\right), 14.7$ $\left(\mathbf{C}_{11}\right)$.

2964 (w), 2915 (w), 2853 (w), 1633 (s), $1566(\mathrm{~m})$, 1422 (s), 1273 (m), 1215 (m), $1111(\mathrm{~s}), 1069$ (m), $1032(\mathrm{~s}), 958(\mathrm{w}), 876(\mathrm{w}), 850(\mathrm{w}), 746(\mathrm{~s}), 689(\mathrm{~m})$, $661(\mathrm{~m}), 579(\mathrm{~m}), 436(\mathrm{~m})$.

$[\mathrm{M}+\mathrm{H}]^{+}$Calcd for $\mathrm{C}_{13} \mathrm{H}_{17} \mathrm{BrNO}_{2} \mathrm{~S}^{+} 330.0158$;

Found 330.0158.

0.49 (UV, CAM). 

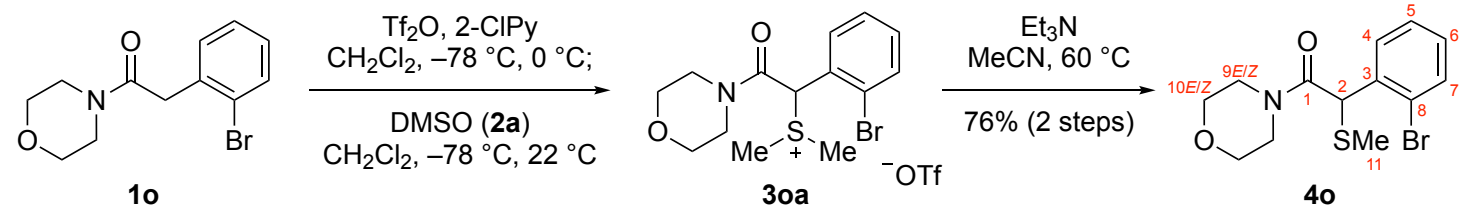

a-SMe-Morpholine Amide 40:

The $\alpha$-SMe-morpholine amide 40 was prepared via Method A using azeotropically dried morpholine amide 10 (142 mg, $500 \mu \mathrm{mol}, 1$ equiv) and dimethyl sulfoxide (2a, $42.6 \mu \mathrm{L}, 600 \mu \mathrm{mol}$, 1.20 equiv). The brown oily crude material was purified via flash column chromatography on silica gel (eluent: 25\% EtOAc in hexanes) to yield $\alpha$-SMe-morpholine amide 40 (126 $\mathrm{mg}, 76 \%$ ) as an oil. Structural assignments were made using additional information from gCOSY, gHSQC and gHMBC experiments.

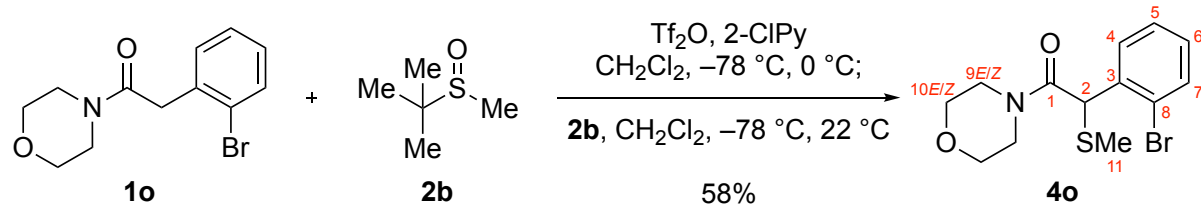

a-SMe-Morpholine Amide 4o:

The $\alpha$-SMe-morpholine amide 40 was prepared via Method B using azeotropically dried morpholine amide 10 (142 mg, $500 \mu \mathrm{mol}, 1$ equiv) and tert-butyl methyl sulfoxide $(\mathbf{2 b}, 69.8 \mu \mathrm{L}$, $600 \mu \mathrm{mol}, 1.20$ equiv). The light yellow oily crude material was purified via flash column chromatography on silica gel (eluent: $25 \%$ EtOAc in hexanes) to yield $\alpha$-SMe-morpholine amide 4o $(96.1 \mathrm{mg}, 58 \%)$ as an oil. The analytic data is fully consistent with that of compound 40 from Method A.

${ }^{1} \mathrm{H}$ NMR (400 MHz, $\left.\mathrm{CDCl}_{3}, 25^{\circ} \mathrm{C}\right)$ :

${ }^{13} \mathrm{C}$ NMR (100 MHz, $\left.\mathrm{CDCl}_{3}, 25^{\circ} \mathrm{C}\right)$ :

FTIR (thin film) $\mathrm{cm}^{-1}$ :

HRMS (DART-TOF+) $m / z$ :

TLC (67\% EtOAc in hexanes), Rf: $\delta 7.70\left(\mathrm{~d}, J=7.8 \mathrm{~Hz}, 1 \mathrm{H}, \mathrm{C}_{7} \mathbf{H}\right), 7.54(\mathrm{~d}, J=8.0 \mathrm{~Hz}$, $\left.1 \mathrm{H}, \mathrm{C}_{4} \mathbf{H}\right), 7.35\left(\mathrm{t}, J=7.6 \mathrm{~Hz}, 1 \mathrm{H}, \mathrm{C}_{5} \mathbf{H}\right), 7.16(\mathrm{t}, J=$ $\left.7.7 \mathrm{~Hz}, 1 \mathrm{H}, \mathrm{C}_{6} \mathbf{H}\right), 5.25\left(\mathrm{~s}, 1 \mathrm{H}, \mathrm{C}_{2} \mathbf{H}\right), 3.70-3.52(\mathrm{~m}$, $\left.6 \mathrm{H}, 3 \times \mathrm{C}_{9 E / Z} \mathbf{H}, 3 \times \mathrm{C}_{10 E / Z} \mathbf{H}\right), 3.43-3.36(\mathrm{~m}, 1 \mathrm{H}$, $\left.\mathrm{C}_{9 E / Z} \mathbf{H}\right), 3.35-3.28\left(\mathrm{~m}, 1 \mathrm{H}, \mathrm{C}_{10 E / Z} \mathbf{H}\right), 2.06(\mathrm{~s}, 3 \mathrm{H}$, $\left.\mathrm{C}_{11} \mathbf{H}_{3}\right)$.

$\delta 167.8\left(\mathbf{C}_{\mathbf{1}}\right), 136.5\left(\mathbf{C}_{3}\right), 132.8\left(\mathbf{C}_{4}\right), 130.8\left(\mathbf{C}_{7}\right)$, $129.6\left(\mathbf{C}_{6}\right), 128.4\left(\mathbf{C}_{5}\right), 124.1\left(\mathbf{C}_{8}\right), 66.8\left(\mathbf{C}_{10 E / Z}\right), 66.5$ $\left(\mathbf{C}_{10 E / Z}\right), 51.1\left(\mathbf{C}_{2}\right), 46.5\left(\mathbf{C}_{9 E / Z}\right), 43.0\left(\mathbf{C}_{9 E / Z}\right), 14.6$ $\left(\mathbf{C}_{11}\right)$.

2963 (w), 2916 (w), 2852 (w), 1641 (s), 1423 (s), $1273(\mathrm{~m}), 1216(\mathrm{~m}), 1111(\mathrm{~s}), 1067$ (s), 850 (m), 747 (s), $577(\mathrm{~m}), 447(\mathrm{~m})$.

$[\mathrm{M}+\mathrm{H}]^{+}$Calcd for $\mathrm{C}_{13} \mathrm{H}_{17} \mathrm{BrNO}_{2} \mathrm{~S}^{+} 330.0158$; Found 330.0155.

0.59 (UV, CAM). 


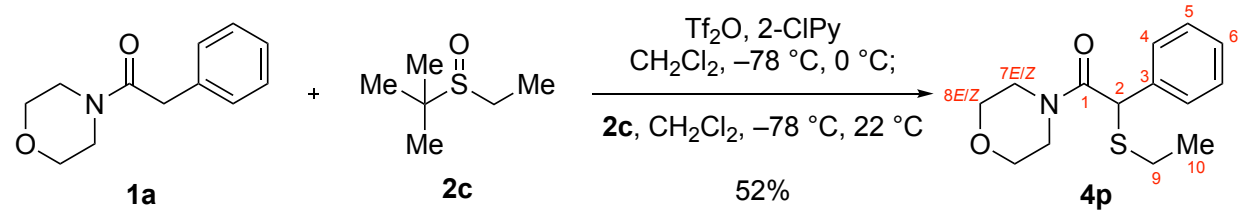

a-SEt-Morpholine Amide 4p:

The $\alpha$-SEt-morpholine amide $4 \mathbf{p}$ was prepared via Method B using azeotropically dried morpholine amide 1a (103 mg, $500 \mu \mathrm{mol}, 1$ equiv) and tert-butyl ethyl sulfoxide (2c, $80.5 \mathrm{mg}$, $600 \mu \mathrm{mol}, 1.20$ equiv). The bright orange oily crude material was purified via flash column chromatography on silica gel (eluent: $20 \%$ EtOAc in hexanes) to yield $\alpha$-SEt-morpholine amide $4 p(68.5 \mathrm{mg}, 52 \%)$ as an oil. Structural assignments were made using additional information from gCOSY, gHSQC and gHMBC experiments.

${ }^{1} \mathrm{H}$ NMR $\left(400 \mathrm{MHz}, \mathrm{CDCl}_{3}, 25^{\circ} \mathrm{C}\right): \quad \delta 7.39\left(\mathrm{~d}, J=6.9 \mathrm{~Hz}, 2 \mathrm{H}, 2 \times \mathrm{C}_{4} \mathbf{H}\right), 7.32(\mathrm{t}, J=$ $\left.7.5 \mathrm{~Hz}, 2 \mathrm{H}, 2 \times \mathrm{C}_{5} \mathbf{H}\right), 7.26\left(\mathrm{t}, J=6.9 \mathrm{~Hz}, 1 \mathrm{H}, \mathrm{C}_{6} \mathbf{H}\right)$, $4.83\left(\mathrm{~s}, 1 \mathrm{H}, \mathrm{C}_{2} \mathbf{H}\right), 3.68-3.28\left(\mathrm{~m}, 8 \mathrm{H}, 4 \times \mathrm{C}_{7 E / Z} \mathbf{H}, 4 \times\right.$ $\left.\mathrm{C}_{8 E / Z} \mathbf{H}\right), 2.52-2.39\left(\mathrm{~m}, 2 \mathrm{H}, \mathrm{C}_{9} \mathbf{H}_{2}\right), 1.19(\mathrm{t}, J=7.4 \mathrm{~Hz}$, $\left.3 \mathrm{H}, \mathrm{C}_{10} \mathbf{H}_{3}\right)$.

${ }^{13} \mathrm{C}$ NMR (100 MHz, $\left.\mathrm{CDCl}_{3}, 25^{\circ} \mathrm{C}\right)$ :

$\delta 168.4\left(\mathbf{C}_{\mathbf{1}}\right), 137.1\left(\mathbf{C}_{\mathbf{3}}\right), 128.8\left(2 \times \mathbf{C}_{\mathbf{5}}\right), 128.2(2 \times$ $\left.\mathbf{C}_{4}\right), 127.9\left(\mathbf{C}_{6}\right), 66.8\left(\mathbf{C}_{8 E / Z}\right), 66.3\left(\mathbf{C}_{8 E / Z}\right), 51.7\left(\mathbf{C}_{2}\right)$, $46.6\left(\mathbf{C}_{7 E / Z}\right), 42.8\left(\mathbf{C}_{7 E / Z}\right), 25.4\left(\mathbf{C}_{9}\right), 14.2\left(\mathbf{C}_{\mathbf{1 0}}\right)$.

FTIR (thin film) $\mathrm{cm}^{-1}$ :

$2964(\mathrm{w}), 2923(\mathrm{w}), 2854(\mathrm{w}), 1634$ (s), $1426(\mathrm{~s})$, $1277(\mathrm{~m}), 1216(\mathrm{~m}), 1112(\mathrm{~s}), 1031(\mathrm{~m}), 964(\mathrm{w}), 852$ (w), 733 (s), $698(\mathrm{~s}), 576(\mathrm{~m})$.

HRMS (DART-TOF+) $m / z$ :

$[\mathrm{M}+\mathrm{H}]^{+}$Calcd for $\mathrm{C}_{14} \mathrm{H}_{20} \mathrm{NO}_{2} \mathrm{~S}^{+}$266.1209;

Found 266.1211.

TLC (67\% EtOAc in hexanes), Rf: 0.49 (UV, CAM). 


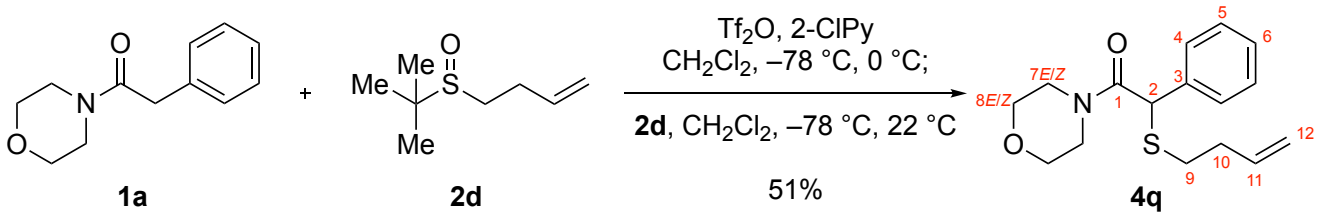

$\alpha$-S(But-3-en-1-yl)-Morpholine Amide 4q:

The $\alpha$-S(but-3-en-1-yl)-morpholine amide $\mathbf{4 q}$ was prepared via Method B using azeotropically dried morpholine amide 1a (103 mg, $500 \mu \mathrm{mol}, 1$ equiv) and tert-butyl 3-butenyl sulfoxide (2d, $96.2 \mathrm{mg}, 600 \mu \mathrm{mol}, 1.20$ equiv). The bright orange oily crude material was purified via flash column chromatography on silica gel (eluent: $25 \%$ EtOAc in hexanes) to yield $\alpha-S$ (but3-en-1-yl)-morpholine amide $\mathbf{4 q}(74.2 \mathrm{mg}, 51 \%)$ as an oil. Structural assignments were made using additional information from gCOSY, gHSQC and gHMBC experiments.

${ }^{1} \mathrm{H}$ NMR (400 MHz, $\left.\mathrm{CDCl}_{3}, 25^{\circ} \mathrm{C}\right)$ :

${ }^{13} \mathrm{C} \mathrm{NMR}\left(100 \mathrm{MHz}, \mathrm{CDCl}_{3}, 25{ }^{\circ} \mathrm{C}\right)$ :

FTIR (thin film) $\mathrm{cm}^{-1}$ :

HRMS (DART-TOF+) $m / z$ :

TLC (67\% EtOAc in hexanes), Rf: $\delta 7.40\left(\mathrm{~d}, J=7.1 \mathrm{~Hz}, 2 \mathrm{H}, 2 \times \mathrm{C}_{4} \mathbf{H}\right), 7.34(\mathrm{~d}, J=$ $\left.7.4 \mathrm{~Hz}, 2 \mathrm{H}, 2 \times \mathrm{C}_{5} \mathbf{H}\right), 7.30-7.26\left(\mathrm{~m}, 1 \mathrm{H}, \mathrm{C}_{6} \mathbf{H}\right), 5.76$ (ddt, $\left.J=16.9,10.2,6.6 \mathrm{~Hz}, 1 \mathrm{H}, \mathrm{C}_{11} \mathbf{H}\right), 5.06-4.97(\mathrm{~m}$, $\left.2 \mathrm{H}, \mathrm{C}_{12} \mathbf{H}_{2}\right), 4.84\left(\mathrm{~s}, 1 \mathrm{H}, \mathrm{C}_{2} \mathbf{H}\right), 3.70-3.55(\mathrm{~m}, 4 \mathrm{H}, 2 \times$ $\left.\mathrm{C}_{7 E / Z} \mathbf{H}, 2 \times \mathrm{C}_{8 E / Z} \mathbf{H}\right), 3.54-3.42\left(\mathrm{~m}, 2 \mathrm{H}, \mathrm{C}_{7 E / Z} \mathbf{H}\right.$, $\left.\mathrm{C}_{8 E / Z} \mathbf{H}\right), 3.40-3.26\left(\mathrm{~m}, 2 \mathrm{H}, \mathrm{C}_{7 E / Z} \mathbf{H}, \mathrm{C}_{8 E / Z} \mathbf{H}\right), 2.57-$ $2.47\left(\mathrm{~m}, 2 \mathrm{H}, \mathrm{C}_{10} \mathbf{H}_{2}\right), 2.34-2.21\left(\mathrm{~m}, 2 \mathrm{H}, \mathrm{C}_{9} \mathbf{H}_{2}\right)$.

$\delta 168.4\left(\mathbf{C}_{\mathbf{1}}\right), 137.0\left(\mathbf{C}_{\mathbf{3}}\right), 136.6\left(\mathbf{C}_{\mathbf{1 1}}\right), 128.9\left(2 \times \mathbf{C}_{\mathbf{5}}\right)$, $128.3\left(2 \times \mathbf{C}_{4}\right), 128.0\left(\mathbf{C}_{6}\right), 116.2\left(\mathbf{C}_{\mathbf{1 2}}\right), 66.8\left(\mathbf{C}_{8 E / Z}\right)$, $66.4\left(\mathbf{C}_{8 E / Z}\right), 52.0\left(\mathbf{C}_{2}\right), 46.6\left(\mathbf{C}_{7 E / Z}\right), 42.9\left(\mathbf{C}_{7 E / Z}\right)$, 33.4 (C) $30.7\left(\mathbf{C}_{\mathbf{1 0}}\right)$.

2962 (w), 2917 (w), $2854(\mathrm{w}), 1623$ (s), 1437 (s), $1222(\mathrm{~m}), 1112(\mathrm{~s}), 1031(\mathrm{~m}), 912(\mathrm{~m}), 849(\mathrm{~m}), 740$ (m), $716(\mathrm{~m}), 696(\mathrm{~m}), 575(\mathrm{~m})$.

$[\mathrm{M}+\mathrm{H}]^{+}$Calcd for $\mathrm{C}_{16} \mathrm{H}_{22} \mathrm{NO}_{2} \mathrm{~S}^{+}$292.1366;

Found 292.1358.

0.51 (UV, CAM). 

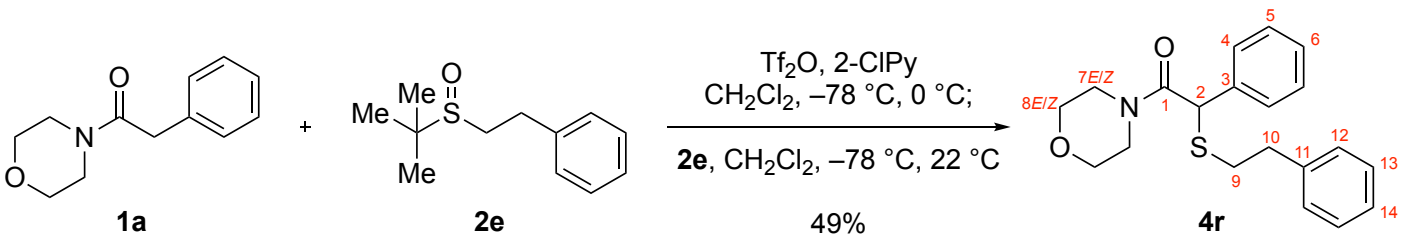

Q-S(2-Phenethyl)-Morpholine Amide 4r:

The $\alpha$-S(2-phenethyl)-morpholine amide $4 \mathbf{r}$ was prepared via Method B using azeotropically dried morpholine amide $1 \mathrm{a}(103 \mathrm{mg}, 500 \mu \mathrm{mol}, 1$ equiv) and tert-butyl 2-phenethyl sulfoxide (2e, $126 \mathrm{mg}, 600 \mu \mathrm{mol}, 1.20$ equiv). The bright orange oily crude material was purified via flash column chromatography on silica gel (eluent: 20\% EtOAc in hexanes) to yield $\alpha-S(2-$ phenethyl)-morpholine amide $4 \mathbf{r}(83.3 \mathrm{mg}, 49 \%)$ as a solid. Structural assignments were made using additional information from gCOSY, gHSQC and gHMBC experiments.

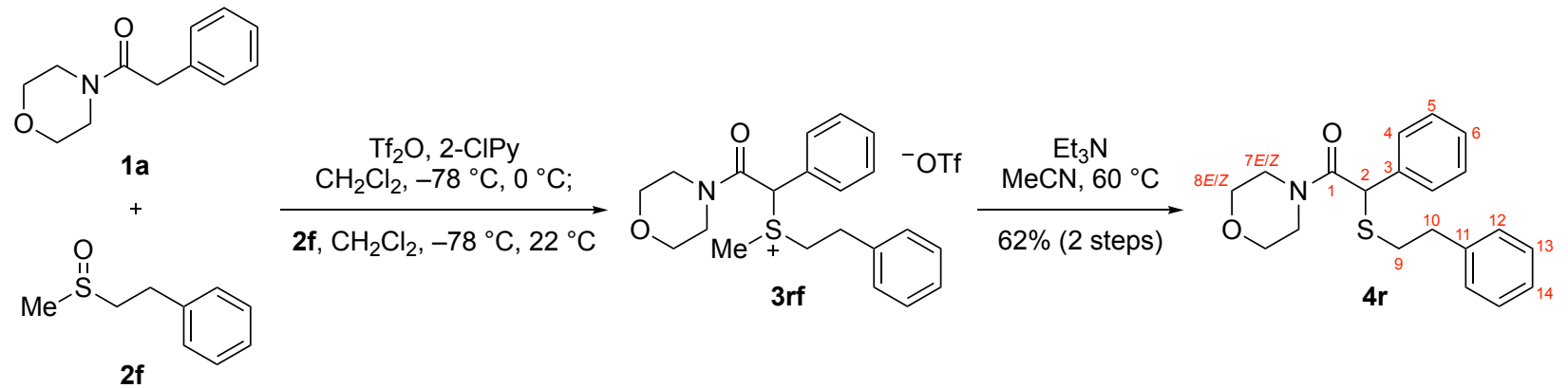

\section{$\alpha$-S(2-Phenethyl)-Morpholine Amide 4r:}

The $\alpha$-S(2-phenethyl)-morpholine amide $4 \mathbf{r}$ was prepared via Method A using azeotropically dried morpholine amide 1a (103 mg, $500 \mu \mathrm{mol}, 1$ equiv) and methyl 2-phenethyl sulfoxide (2f, $101 \mathrm{mg}, 600 \mu \mathrm{mol}, 1.20$ equiv). The brown oily crude material was purified via flash column chromatography on silica gel (eluent: $25 \%$ EtOAc in hexanes) to yield $\alpha$-S(2-phenethyl)morpholine amide $4 \mathbf{r}(106 \mathrm{mg}, 62 \%)$ as an oil. The analytic data is fully consistent with that of compound 4r from Method B.

${ }^{1} \mathrm{H}$ NMR $\left(400 \mathrm{MHz}, \mathrm{CDCl}_{3}, 25^{\circ} \mathrm{C}\right): \quad \delta 7.30-7.25\left(\mathrm{~m}, 3 \mathrm{H}, 2 \times \mathrm{C}_{4} \mathbf{H}, \mathrm{C}_{6} \mathbf{H}\right), 7.24-7.18(\mathrm{~m}$, $\left.4 \mathrm{H}, 2 \times \mathrm{C}_{5} \mathbf{H}, 2 \times \mathrm{C}_{13} \mathbf{H}\right), 7.15-7.11\left(\mathrm{~m}, 1 \mathrm{H}, \mathrm{C}_{14} \mathbf{H}\right)$, 7.10-7.07 (m, 2H, $\left.2 \times \mathrm{C}_{12} \mathbf{H}\right), 4.60\left(\mathrm{~s}, 1 \mathrm{H}, \mathrm{C}_{2} \mathbf{H}\right)$, $3.59-3.45\left(\mathrm{~m}, 4 \mathrm{H}, 2 \times \mathrm{C}_{7 E / Z} \mathbf{H}, 2 \times \mathrm{C}_{8 E / Z} \mathbf{H}\right), 3.42-3.34$ $\left(\mathrm{m}, 1 \mathrm{H}, \mathrm{C}_{8 E / Z} \mathbf{H}\right), 3.28-3.21\left(\mathrm{~m}, 1 \mathrm{H}, \mathrm{C}_{7 E / Z} \mathbf{H}\right), 3.19-$ $3.11\left(\mathrm{~m}, 2 \mathrm{H}, \mathrm{C}_{7 E / Z} \mathbf{H}, \mathrm{C}_{8 E / Z} \mathbf{H}\right), 2.75(\mathrm{t}, J=7.2 \mathrm{~Hz}, 2 \mathrm{H}$, $\left.\mathrm{C}_{10} \mathbf{H}_{2}\right), 2.69-2.57\left(\mathrm{~m}, 2 \mathrm{H}, \mathrm{C}_{9} \mathbf{H}_{2}\right)$.

${ }^{13} \mathrm{C}$ NMR $\left(100 \mathrm{MHz}, \mathrm{CDCl}_{3}, 25{ }^{\circ} \mathrm{C}\right)$ :

$\delta 168.3\left(\mathbf{C}_{\mathbf{1}}\right), 140.5\left(\mathbf{C}_{\mathbf{1 1}}\right), 137.0\left(\mathbf{C}_{\mathbf{3}}\right), 128.9\left(2 \times \mathbf{C}_{\mathrm{Ar}}\right)$, $128.7\left(2 \times \mathbf{C}_{\mathbf{A r}}\right), 128.6\left(2 \times \mathbf{C}_{\mathbf{A r}}\right), 128.4\left(2 \times \mathbf{C}_{4}\right)$, $128.1\left(\mathbf{C}_{\mathbf{6}}\right), 126.5\left(\mathbf{C}_{\mathbf{1 4}}\right), 66.8\left(\mathbf{C}_{\mathbf{8 E} / \mathbf{Z}}\right), 66.3\left(\mathbf{C}_{\mathbf{8 E / Z}}\right)$, $52.0\left(\mathbf{C}_{2}\right), 46.5\left(\mathbf{C}_{\mathbf{7 E / Z}}\right), 42.8\left(\mathbf{C}_{\mathbf{7 E / Z} / \mathbf{Z}}\right), 36.1\left(\mathbf{C}_{\mathbf{1 0}}\right), 32.6$ (C9).

FTIR (thin film) $\mathrm{cm}^{-1}$ : $2918(\mathrm{w}), 2857(\mathrm{w}), 1623$ (s), 1434 (m), 1223 (m), 1115 (s), 1033 (m), 855 (w), 738 (m), 700 (m), 574 (m), 497 (m). 
HRMS (DART-TOF+) $m / z$ :

TLC (67\% EtOAc in hexanes), Rf:

M.p.:
$[\mathrm{M}+\mathrm{H}]^{+}$Calcd for $\mathrm{C}_{20} \mathrm{H}_{24} \mathrm{NO}_{2} \mathrm{~S}^{+} 342.1522$;

Found 342.1521.

0.53 (UV, CAM).

$82-84{ }^{\circ} \mathrm{C}\left(\mathrm{CH}_{2} \mathrm{Cl}_{2}\right)$. 

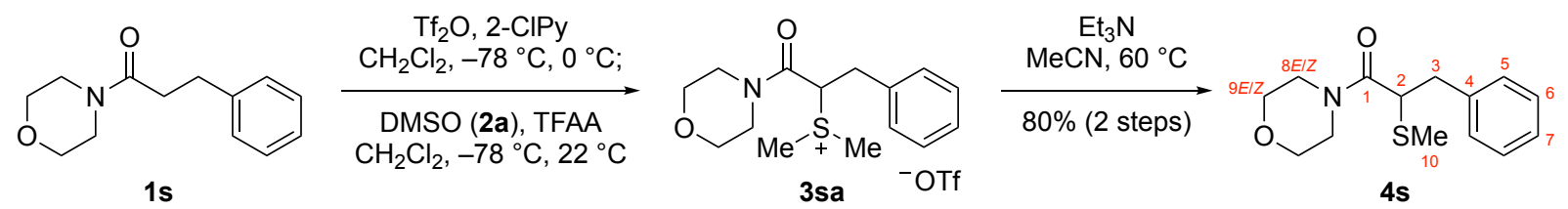

Method C: Representative Procedure for the $\alpha$-Sulfidation of Aliphatic Amides with Methyl Sulfoxides. $\alpha$-SMe-Morpholine Amide 4s:

Trifluoromethanesulfonic anhydride $(92.5 \mu \mathrm{L}, 550 \mu \mathrm{mol}, 1.10$ equiv) was added via syringe over $1 \mathrm{~min}$ to a stirred solution of azeotropically dried morpholine amide $1 \mathrm{~s}$ (110 $\mathrm{mg}$, $500 \mu \mathrm{mol}, 1$ equiv) and 2-chloropyridine $(142 \mu \mathrm{L}, 1.50 \mathrm{mmol}, 3.00$ equiv) in dichloromethane $(1.7 \mathrm{~mL})$ at $-78^{\circ} \mathrm{C}$ under argon atmosphere. The bright red solution was stirred at $0{ }^{\circ} \mathrm{C}$ for $15 \mathrm{~min}$, before it was again cooled to $-78^{\circ} \mathrm{C}$. A solution of dimethyl sulfoxide $(\mathbf{2 a}, 88.8 \mu \mathrm{L}, 1.25 \mathrm{mmol}$, 2.50 equiv) in dichloromethane $(0.3 \mathrm{~mL})$ was added via cannula over $2 \mathrm{~min}$, followed by the addition of trifluoroacetic anhydride $(69.5 \mu \mathrm{L}, 500 \mu \mathrm{mol}, 1.00$ equiv) via syringe over 1 min at $78{ }^{\circ} \mathrm{C}$. The reaction mixture was stirred at $0{ }^{\circ} \mathrm{C}$ for $15 \mathrm{~min}$, then at $22{ }^{\circ} \mathrm{C}$ for $30 \mathrm{~min}$ to give a yellow solution. A saturated aqueous solution of sodium bicarbonate $(2.0 \mathrm{~mL})$ followed by dichloromethane $(1.0 \mathrm{~mL})$ was added, and the yellow biphasic mixture was vigorously stirred at $22{ }^{\circ} \mathrm{C}$ for $15 \mathrm{~min}$. After separation of the organic layer, the aqueous phase was extracted with dichloromethane $(3 \times 1.0 \mathrm{~mL})$. The combined light yellow organic extracts were dried over sodium sulfate, were filtered, and were concentrated under reduced pressure. The yellow oily residue was dissolved in acetonitrile $(2.0 \mathrm{~mL})$. Triethylamine $(697 \mu \mathrm{L}, 5.00 \mathrm{mmol}, 10.0$ equiv) was added, and the resulting brown solution was stirred in a sealed roundbottom flask at $60{ }^{\circ} \mathrm{C}$ for $15 \mathrm{~h}$. After concentration under reduced pressure, the brown oily crude material was purified via flash column chromatography on silica gel (eluent: $25 \%$ EtOAc in hexanes) to yield $\alpha$-SMe-morpholine amide 4s (106 mg, 80\%) as a solid. ${ }^{15}$ Structural assignments were made using additional information from gCOSY, gHSQC and gHMBC experiments.

${ }^{1} \mathrm{H}$ NMR $\left(400 \mathrm{MHz}, \mathrm{CDCl}_{3}, 25^{\circ} \mathrm{C}\right)$ :

${ }^{13} \mathrm{C}$ NMR (100 MHz, $\left.\mathrm{CDCl}_{3}, 25{ }^{\circ} \mathrm{C}\right)$ :

FTIR (thin film) $\mathrm{cm}^{-1}$ :

HRMS (DART-TOF+) $m / z$ :

TLC (67\% EtOAc in hexanes), Rf:

M.p.: $\delta$ 7.32-7.28 (m, 2H, $\left.2 \times \mathrm{C}_{5} \mathbf{H}\right), 7.25-7.22(\mathrm{~m}, 3 \mathrm{H}, 2 \times$ $\left.\mathrm{C}_{6} \mathbf{H}, \mathrm{C}_{7} \mathbf{H}\right), 3.69-3.47\left(\mathrm{~m}, 7 \mathrm{H}, \mathrm{C}_{2} \mathbf{H}, 3 \times \mathrm{C}_{8 E / Z} \mathbf{H}, 3 \times\right.$ $\left.\mathrm{C}_{9 E / Z} \mathbf{H}\right), 3.42-3.32\left(\mathrm{~m}, 2 \mathrm{H}, \mathrm{C}_{3} \mathbf{H}_{\mathbf{a}}, \mathrm{C}_{9 E / Z} \mathbf{H}\right), 3.30-3.24$ $\left(\mathrm{m}, 1 \mathrm{H}, \mathrm{C}_{8 E / Z} \mathbf{H}\right), 3.02(\mathrm{dd}, J=13.6,6.0 \mathrm{~Hz}, 1 \mathrm{H}$, $\left.\mathrm{C}_{3} \mathbf{H}_{\mathbf{b}}\right), 2.16\left(\mathrm{~s}, 3 \mathrm{H}, \mathrm{C}_{10} \mathbf{H}_{3}\right)$.

$\delta 168.8\left(\mathbf{C}_{\mathbf{1}}\right), 138.9\left(\mathbf{C}_{\mathbf{4}}\right), 129.3\left(2 \times \mathbf{C}_{\mathbf{5}}\right), 128.5(2 \times$ $\left.\mathbf{C}_{6}\right), 126.7\left(\mathbf{C}_{7}\right), 67.0\left(\mathbf{C}_{9 E / Z}\right), 66.6\left(\mathbf{C}_{9 E / Z}\right), 46.5$ $\left(\mathbf{C}_{8 E / Z}\right), 44.2\left(\mathbf{C}_{2}\right), 42.5\left(\mathbf{C}_{8 E / Z}\right), 37.6\left(\mathbf{C}_{3}\right), 12.2\left(\mathbf{C}_{10}\right)$.

$2958(\mathrm{w}), 2922(\mathrm{w}), 2863(\mathrm{w}), 1616(\mathrm{~s}), 1463(\mathrm{~m})$, 1439 (m), 1225 (m), 1112 (s), 752 (m), 720 (m), 575 (m), $506(\mathrm{~m})$.

$[\mathrm{M}+\mathrm{H}]^{+}$Calcd for $\mathrm{C}_{14} \mathrm{H}_{20} \mathrm{NO}_{2} \mathrm{~S}^{+}$266.1209;

Found 266.1204.

0.58 (UV, CAM).

$73-75{ }^{\circ} \mathrm{C}\left(\mathrm{CH}_{2} \mathrm{Cl}_{2}\right)$. 

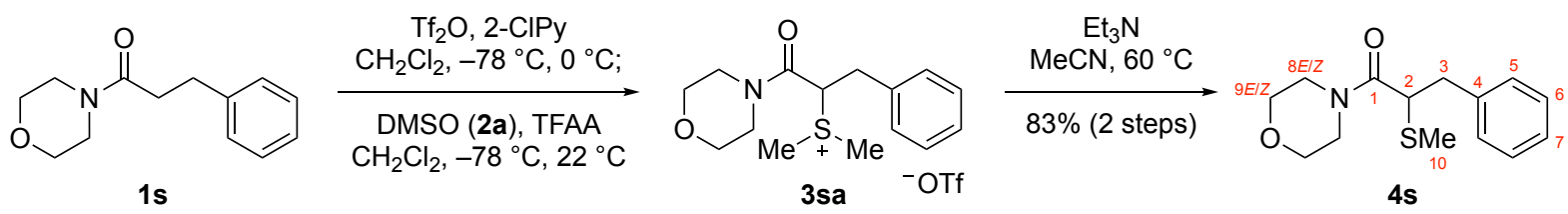

\section{a-SMe-Morpholine Amide 4s $(5.00$ mmol scale):}

Trifluoromethanesulfonic anhydride $(925 \mu \mathrm{L}, 5.50 \mathrm{mmol}, 1.10$ equiv) was added via syringe over $1 \mathrm{~min}$ to a stirred solution of azeotropically dried morpholine amide $1 \mathrm{~s}(1.10 \mathrm{~g}$, $5.00 \mathrm{mmol}, 1$ equiv) and 2-chloropyridine (1.42 $\mathrm{mL}, 15.0 \mathrm{mmol}, 3.00$ equiv) in dichloromethane $(17 \mathrm{~mL})$ at $-78^{\circ} \mathrm{C}$ under argon atmosphere. The bright red solution was stirred at $0{ }^{\circ} \mathrm{C}$ for $15 \mathrm{~min}$, before it was again cooled to $-78^{\circ} \mathrm{C}$. A solution of dimethyl sulfoxide $(\mathbf{2 a}, 888 \mu \mathrm{L}, 12.5 \mathrm{mmol}$, 2.50 equiv) in dichloromethane $(3.0 \mathrm{~mL})$ was added via cannula over $2 \mathrm{~min}$, followed by the addition of trifluoroacetic anhydride $(695 \mu \mathrm{L}, 5.00 \mathrm{mmol}, 1.00$ equiv) via syringe over $1 \mathrm{~min}$ at $-78{ }^{\circ} \mathrm{C}$. The reaction mixture was stirred at $0{ }^{\circ} \mathrm{C}$ for $15 \mathrm{~min}$, then at $22{ }^{\circ} \mathrm{C}$ for $30 \mathrm{~min}$ to give a yellow solution. A saturated aqueous solution of sodium bicarbonate $(20 \mathrm{~mL})$ followed by dichloromethane $(10 \mathrm{~mL})$ was added, and the yellow biphasic mixture was vigorously stirred at $22{ }^{\circ} \mathrm{C}$ for $15 \mathrm{~min}$. After separation of the organic layer, the aqueous phase was extracted with dichloromethane $(3 \times 10 \mathrm{~mL})$. The combined light yellow organic extracts were dried over sodium sulfate, were filtered, and were concentrated under reduced pressure. The yellow oily residue was dissolved in acetonitrile $(20 \mathrm{~mL})$. Triethylamine $(6.97 \mathrm{~mL}, 50.0 \mathrm{mmol}, 10.0$ equiv) was added, and the resulting brown solution was stirred in a sealed roundbottom flask at $60{ }^{\circ} \mathrm{C}$ for $15 \mathrm{~h}$. After concentration under reduced pressure, the brown oily crude material was purified via flash column chromatography on silica gel (eluent: $25 \%$ EtOAc in hexanes) to yield $\alpha$-SMe-morpholine amide 4s $(1.10 \mathrm{~g}, 83 \%)$ as a solid. ${ }^{15}$ The analytic data is fully consistent with that of compound $4 \mathbf{s}$ as described on a previous page of this document. 

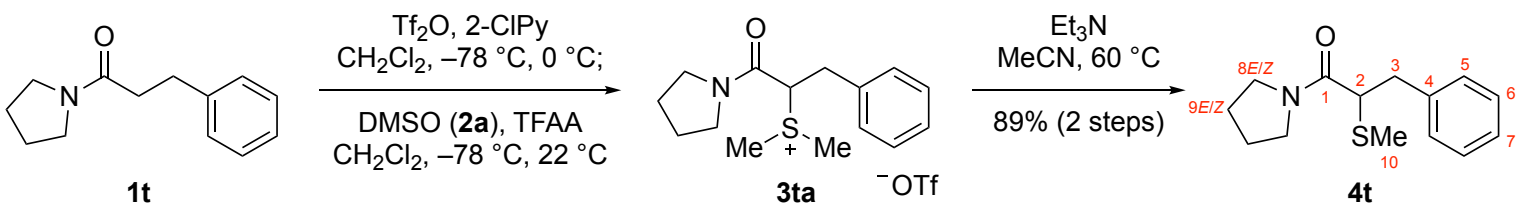

\section{$\alpha$-SMe-Pyrrolidine Amide 4t:}

The $\alpha$-SMe-pyrrolidine amide $4 \mathbf{t}$ was prepared via Method $\mathrm{C}$ using azeotropically dried pyrrolidine amide 1t $(102 \mathrm{mg}, 500 \mu \mathrm{mol}, 1$ equiv) and dimethyl sulfoxide (2a, $88.8 \mu \mathrm{L}, 1.25 \mathrm{mmol}$, 2.50 equiv). The brown oily crude material was purified via flash column chromatography on silica gel (eluent: $25 \%$ EtOAc in hexanes) to yield $\alpha$-SMe-pyrrolidine amide $4 \mathbf{t}(111 \mathrm{mg}, 89 \%)$ as a solid. ${ }^{15}$ Structural assignments were made using additional information from gCOSY, gHSQC and gHMBC experiments.

${ }^{1} \mathrm{H}$ NMR (400 MHz, $\left.\mathrm{CDCl}_{3}, 25^{\circ} \mathrm{C}\right)$ :

${ }^{13} \mathrm{C} \mathrm{NMR}\left(100 \mathrm{MHz}, \mathrm{CDCl}_{3}, 25^{\circ} \mathrm{C}\right)$ :

FTIR (thin film) $\mathrm{cm}^{-1}$ :

HRMS (DART-TOF+) $m / z$ :

TLC (67\% EtOAc in hexanes), Rf:

M.p.: $\delta$ 7.31-7.20 (m, 5H, $\left.2 \times \mathrm{C}_{5} \mathbf{H}, 2 \times \mathrm{C}_{6} \mathbf{H}, \mathrm{C}_{7} \mathbf{H}\right), 3.59$ $3.53\left(\mathrm{~m}, 1 \mathrm{H}, \mathrm{C}_{8 E / Z} \mathbf{H}\right), 3.51-3.36\left(\mathrm{~m}, 4 \mathrm{H}, 2 \times \mathrm{C}_{8 E / Z} \mathbf{H}\right.$, $\left.\mathrm{C}_{2} \mathbf{H}, \mathrm{C}_{3} \mathbf{H}_{\mathbf{a}}\right), 3.08\left(\mathrm{dt}, J=9.7,6.5 \mathrm{~Hz}, 1 \mathrm{H}, \mathrm{C}_{8 E / Z} \mathbf{H}\right)$, 2.99 (dd, $\left.J=13.5,5.9 \mathrm{~Hz}, 1 \mathrm{H}, \mathrm{C}_{3} \mathbf{H}_{\mathbf{b}}\right), 2.17$ (s, 3H, $\left.\mathrm{C}_{10} \mathbf{H}_{3}\right), 1.93-1.71\left(\mathrm{~m}, 4 \mathrm{H}, 4 \times \mathrm{C}_{9 E / Z} \mathbf{H}\right)$.

$\delta 168.7\left(\mathbf{C}_{\mathbf{1}}\right), 139.2\left(\mathbf{C}_{4}\right), 129.2\left(2 \times \mathbf{C}_{5}\right), 128.4(2 \times$ $\left.\mathbf{C}_{6}\right), 126.6\left(\mathbf{C}_{7}\right), 46.8\left(\mathbf{C}_{2}\right), 46.3\left(\mathbf{C}_{8 E / Z}\right), 46.0\left(\mathbf{C}_{8 E / Z}\right)$, $37.4\left(\mathbf{C}_{3}\right), 26.1\left(\mathbf{C}_{9 E / Z}\right), 24.3\left(\mathbf{C}_{9 E / Z}\right), 12.2\left(\mathbf{C}_{10}\right)$.

$3036(\mathrm{w}), 2954(\mathrm{w}), 2873(\mathrm{w}), 1636(\mathrm{~s}), 1497(\mathrm{w})$, 1428 (s), 1342 (w), 1320 (w), 743 (m), 696 (s), 532 (m).

$[\mathrm{M}+\mathrm{H}]^{+}$Calcd for $\mathrm{C}_{14} \mathrm{H}_{20} \mathrm{NOS}^{+} 250.1260$;

Found 250.1255.

0.54 (UV, CAM).

$45-47^{\circ} \mathrm{C}\left(\mathrm{CH}_{2} \mathrm{Cl}_{2}\right)$. 


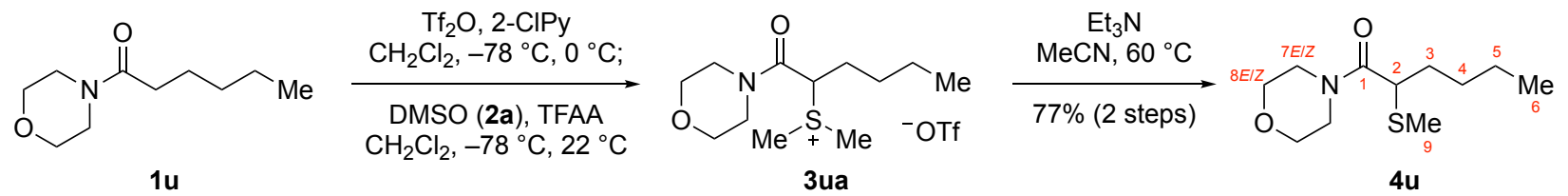

a-SMe-Morpholine Amide 4u:

The $\alpha$-SMe-morpholine amide $\mathbf{4} \mathbf{u}$ was prepared via Method $\mathrm{C}$ using azeotropically dried morpholine amide $1 \mathbf{u}(92.6 \mathrm{mg}, 500 \mu \mathrm{mol}, 1$ equiv) and dimethyl sulfoxide $(\mathbf{2 a}, 88.8 \mu \mathrm{L}$, $1.25 \mathrm{mmol}, 2.50$ equiv). The brown oily crude material was purified via flash column chromatography on silica gel (eluent: $20 \%$ EtOAc in hexanes) to yield $\alpha$-SMe-morpholine amide 4u (88.9 mg, 77\%) as an oil. ${ }^{15}$ Structural assignments were made using additional information from gCOSY, gHSQC and gHMBC experiments.

${ }^{1} \mathrm{H}$ NMR (400 MHz, $\left.\mathrm{CDCl}_{3}, 25^{\circ} \mathrm{C}\right)$ :

${ }^{13} \mathrm{C} \mathrm{NMR}\left(100 \mathrm{MHz}, \mathrm{CDCl}_{3}, 25{ }^{\circ} \mathrm{C}\right)$ :

FTIR (thin film) $\mathrm{cm}^{-1}$ :

HRMS (DART-TOF+) $m / z$ :

TLC (67\% EtOAc in hexanes), Rf:
反 3.76-3.55 (m, 6H, $\left.2 \times \mathrm{C}_{7 E / Z} \mathbf{H}, 4 \times \mathrm{C}_{8 E / Z} \mathbf{H}\right), 3.50$ $3.39\left(\mathrm{~m}, 2 \mathrm{H}, 2 \times \mathrm{C}_{7 E / Z} \mathbf{H}\right), 3.29(\mathrm{t}, J=7.3 \mathrm{~Hz}, 1 \mathrm{H}$, $\left.\mathrm{C}_{2} \mathbf{H}\right), 2.00\left(\mathrm{~s}, 3 \mathrm{H}, \mathrm{C}_{9} \mathbf{H}_{3}\right), 1.98-1.90\left(\mathrm{~m}, 1 \mathrm{H}, \mathrm{C}_{3} \mathbf{H}_{\mathbf{a}}\right)$, 1.73-1.64 (m, 1H, $\left.\mathrm{C}_{3} \mathbf{H}_{\mathbf{b}}\right), 1.39-1.21\left(\mathrm{~m}, 4 \mathrm{H}, \mathrm{C}_{4} \mathbf{H}_{2}\right.$, $\left.\mathrm{C}_{5} \mathbf{H}_{2}\right), 0.87\left(\mathrm{t}, J=6.8 \mathrm{~Hz}, 3 \mathrm{H}, \mathrm{C}_{6} \mathbf{H}_{3}\right)$.

$\delta 169.1\left(\mathbf{C}_{\mathbf{1}}\right), 67.1\left(\mathbf{C}_{\mathbf{8 E} / Z}\right), 66.7\left(\mathbf{C}_{8 E / Z}\right), 46.4\left(\mathbf{C}_{7 E / Z}\right)$, $42.4\left(\mathbf{C}_{7 E / Z}\right), 42.2\left(\mathbf{C}_{2}\right), 30.3\left(\mathbf{C}_{3}\right), 29.6\left(\mathbf{C}_{\mathbf{4}}\right), 22.6$ (C5), 14.0 ( $\left.\mathbf{C}_{6}\right), 11.4\left(\mathbf{C}_{9}\right)$.

$2956(\mathrm{w}), 2922(\mathrm{w}), 2855(\mathrm{w}), 1635$ (s), 1428 (s), 1235 (m), 1113 (s), 1025 (m), 848 (w), 569 (w).

$[\mathrm{M}+\mathrm{H}]^{+}$Calcd for $\mathrm{C}_{11} \mathrm{H}_{22} \mathrm{NO}_{2} \mathrm{~S}^{+}$232.1366;

Found 232.1360.

0.62 (UV, CAM). 
<smiles>C#CCCCC(=O)N1CCOCC1</smiles>

1v

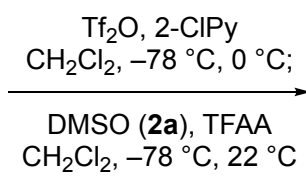

$$
\mathrm{CH}_{2} \mathrm{Cl}_{2},-78^{\circ} \mathrm{C}, 22^{\circ} \mathrm{C}
$$
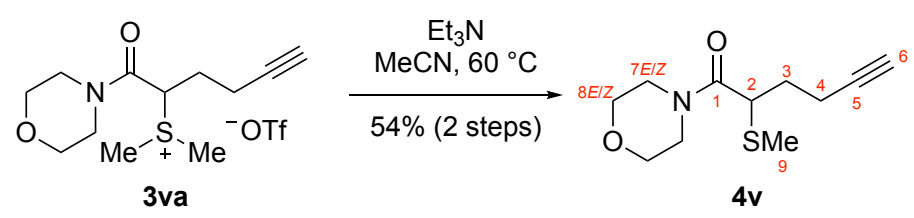

$\underline{\alpha-S M e-M o r p h o l i n e ~ A m i d e ~ 4 v: ~}$

The $\alpha$-SMe-morpholine amide $\mathbf{4} \mathbf{v}$ was prepared via Method $\mathrm{C}$ using azeotropically dried morpholine amide $1 \mathbf{v}(90.6 \mathrm{mg}, 500 \mu \mathrm{mol}, 1$ equiv) and dimethyl sulfoxide $(\mathbf{2 a}, 88.8 \mu \mathrm{L}$, $1.25 \mathrm{mmol}, 2.50$ equiv). The brown oily crude material was purified via flash column chromatography on silica gel (eluent: $20 \%$ EtOAc in hexanes) to yield $\alpha$-SMe-morpholine amide $4 \mathbf{v}(61.0 \mathrm{mg}, 54 \%)$ as a solid. Structural assignments were made using additional information from gCOSY, gHSQC and gHMBC experiments.

${ }^{1} \mathrm{H}$ NMR $\left(400 \mathrm{MHz}, \mathrm{CDCl}_{3}, 25^{\circ} \mathrm{C}\right)$ :

${ }^{13} \mathrm{C} \mathrm{NMR}\left(100 \mathrm{MHz}, \mathrm{CDCl}_{3}, 25^{\circ} \mathrm{C}\right)$ :

FTIR (thin film) $\mathrm{cm}^{-1}$ :

HRMS (DART-TOF+) $m / z$ :

TLC (67\% EtOAc in hexanes), Rf:

M.p.: $\delta$ 3.78-3.67 (m, 4H, $\left.\mathrm{C}_{7 E / Z} \mathbf{H}, 3 \times \mathrm{C}_{8 E / Z} \mathbf{H}\right), 3.64-3.59$ $\left(\mathrm{m}, 3 \mathrm{H}, \mathrm{C}_{2} \mathbf{H}, \mathrm{C}_{7 E / Z} \mathbf{H}, \mathrm{C}_{8 E / Z} \mathbf{H}\right), 3.52-3.43(\mathrm{~m}, 2 \mathrm{H}, 2 \times$ $\left.\mathrm{C}_{7 E / Z} \mathbf{H}\right), 2.43-2.35\left(\mathrm{~m}, 1 \mathrm{H}, \mathrm{C}_{4} \mathbf{H}_{\mathbf{a}}\right), 2.30-2.21(\mathrm{~m}, 1 \mathrm{H}$, $\left.\mathrm{C}_{4} \mathbf{H}_{\mathbf{b}}\right), 2.19-2.12\left(\mathrm{~m}, 1 \mathrm{H}, \mathrm{C}_{3} \mathbf{H}_{\mathbf{a}}\right), 2.01\left(\mathrm{~s}, 3 \mathrm{H}, \mathrm{C}_{9} \mathbf{H}_{3}\right)$, $1.96\left(\mathrm{t}, J=2.5 \mathrm{~Hz}, 1 \mathrm{H}, \mathrm{C}_{6} \mathbf{H}\right), 1.94-1.83(\mathrm{~m}, 1 \mathrm{H}$, $\mathrm{C}_{3} \mathbf{H}_{\mathbf{b}}$ ).

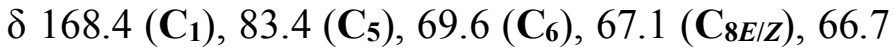
$\left(\mathbf{C}_{8 E / Z}\right), 46.4\left(\mathbf{C}_{7 E / Z}\right), 42.4\left(\mathbf{C}_{7 E / Z}\right), 40.3\left(\mathbf{C}_{2}\right), 29.0$ $\left(\mathbf{C}_{3}\right), 16.2\left(\mathbf{C}_{4}\right), 11.4\left(\mathbf{C}_{9}\right)$.

$3292(\mathrm{w}), 2973(\mathrm{w}), 2914(\mathrm{w}), 2866(\mathrm{w}), 2118(\mathrm{w})$, 1620 (s), 1431 (s), 1245 (s), 1115 (s), 1019 (m), 856 (m), $611(\mathrm{~m}), 550(\mathrm{~m})$.

$[\mathrm{M}+\mathrm{H}]^{+}$Calcd for $\mathrm{C}_{11} \mathrm{H}_{18} \mathrm{NO}_{2} \mathrm{~S}^{+} 228.1053$;

Found 228.1047.

0.55 (UV, CAM).

$46-48{ }^{\circ} \mathrm{C}\left(\mathrm{CH}_{2} \mathrm{Cl}_{2}\right)$. 

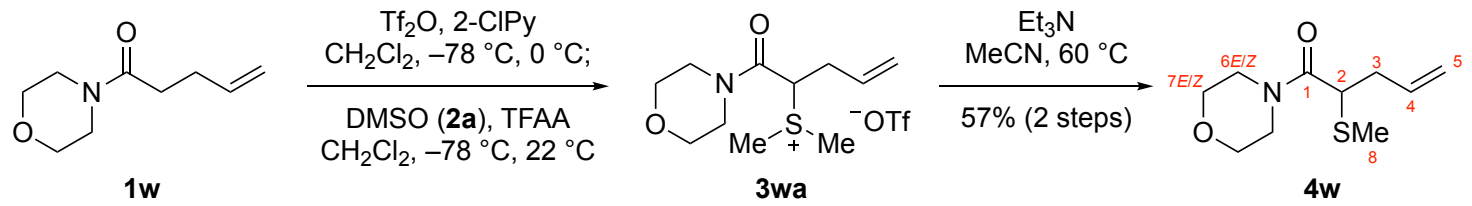

\section{a-SMe-Morpholine Amide 4w:}

The $\alpha$-SMe-morpholine amide $\mathbf{4 w}$ was prepared via Method $\mathrm{C}$ using azeotropically dried morpholine amide $\mathbf{1 w}(84.6 \mathrm{mg}, 500 \mu \mathrm{mol}, 1$ equiv) and dimethyl sulfoxide $(\mathbf{2 a}, 88.8 \mu \mathrm{L}$, $1.25 \mathrm{mmol}, 2.50$ equiv). The brown oily crude material was purified via flash column chromatography on silica gel (eluent: $25 \%$ EtOAc in hexanes) to yield $\alpha$-SMe-morpholine amide $\mathbf{4 w}(61.2 \mathrm{mg}, 57 \%)$ as an oil. Structural assignments were made using additional information from gCOSY, gHSQC and gHMBC experiments.

${ }^{1} \mathrm{H}$ NMR $\left(400 \mathrm{MHz}, \mathrm{CDCl}_{3}, 25^{\circ} \mathrm{C}\right)$ :

${ }^{13} \mathrm{C} \mathrm{NMR}\left(100 \mathrm{MHz}, \mathrm{CDCl}_{3}, 25{ }^{\circ} \mathrm{C}\right)$ :

FTIR (thin film) $\mathrm{cm}^{-1}$ :

HRMS (DART-TOF+) $m / z$ :

TLC (67\% EtOAc in hexanes), Rf: $\delta 5.79$ (ddt, $\left.J=17.1,10.2,6.9 \mathrm{~Hz}, 1 \mathrm{H}, \mathrm{C}_{4} \mathbf{H}\right), 5.09$ $\left(\mathrm{dd}, J=17.2,1.6 \mathrm{~Hz}, 1 \mathrm{H}, \mathrm{C}_{5} \mathbf{H}_{\mathrm{a}}\right), 5.04(\mathrm{~d}, J=10.1 \mathrm{~Hz}$, $\left.1 \mathrm{H}, \mathrm{C}_{5} \mathbf{H}_{\mathbf{b}}\right), 3.77-3.56\left(\mathrm{~m}, 6 \mathrm{H}, 2 \times \mathrm{C}_{6 E / Z} \mathbf{H}, 4 \times\right.$ $\left.\mathrm{C}_{7 E / Z} \mathbf{H}\right), 3.50-3.35\left(\mathrm{~m}, 3 \mathrm{H}, \mathrm{C}_{2} \mathbf{H}, 2 \times \mathrm{C}_{6 E / Z} \mathbf{H}\right), 2.73$ $\left(\mathrm{dt}, J=14.3,7.1 \mathrm{~Hz}, 1 \mathrm{H}, \mathrm{C}_{3} \mathbf{H}_{\mathbf{a}}\right), 2.45$ (dt, $J=14.3$, $\left.7.1 \mathrm{~Hz}, 1 \mathrm{H}, \mathrm{C}_{3} \mathbf{H}_{\mathbf{b}}\right), 2.03\left(\mathrm{~s}, 3 \mathrm{H}, \mathrm{C}_{8} \mathbf{H}_{3}\right)$.

$\delta 168.5\left(\mathbf{C}_{\mathbf{1}}\right), 135.3\left(\mathbf{C}_{\mathbf{4}}\right), 117.3\left(\mathbf{C}_{\mathbf{5}}\right), 67.1\left(\mathbf{C}_{\mathbf{7 E / Z}}\right)$, $66.7\left(\mathbf{C}_{7 E / Z}\right), 46.4\left(\mathbf{C}_{6 E / Z}\right), 42.4\left(\mathbf{C}_{6 E / Z}\right), 41.9\left(\mathbf{C}_{2}\right)$, $34.9\left(\mathbf{C}_{3}\right), 11.4\left(\mathbf{C}_{8}\right)$.

$2964(\mathrm{w}), 2920(\mathrm{w}), 2854(\mathrm{w}), 1632$ (s), 1429 (s), 1240 (m), 1112 (s), 1023 (m), 916 (m), 847 (m), 569 (m).

$[\mathrm{M}+\mathrm{H}]^{+}$Calcd for $\mathrm{C}_{10} \mathrm{H}_{18} \mathrm{NO}_{2} \mathrm{~S}^{+}$216.1053;

Found 216.1048.

0.55 (UV, CAM). 


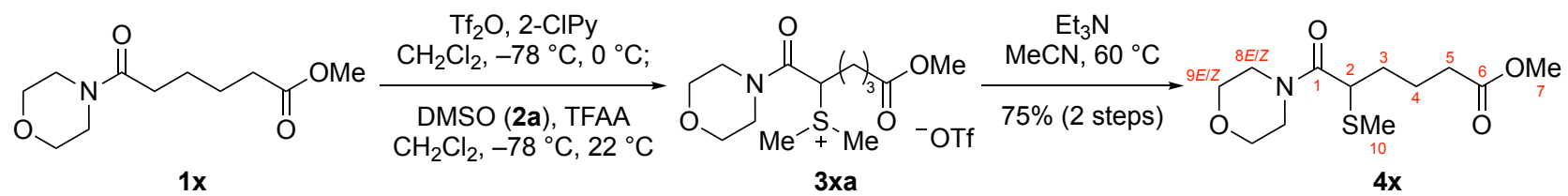

\section{a-SMe-Morpholine Amide 4x:}

The $\alpha$-SMe-morpholine amide $\mathbf{4 x}$ was prepared via Method $\mathrm{C}$ using azeotropically dried morpholine amide $\mathbf{1 x}(115 \mathrm{mg}, 500 \mu \mathrm{mol}, 1$ equiv) and dimethyl sulfoxide $(\mathbf{2 a}, 88.8 \mu \mathrm{L}$, $1.25 \mathrm{mmol}, 2.50$ equiv). The brown oily crude material was purified via flash column chromatography on silica gel (eluent: $25 \%$ EtOAc in hexanes) to yield $\alpha$-SMe-morpholine amide 4x (103 mg, 75\%) as an oil. ${ }^{15}$ Structural assignments were made using additional information from gCOSY, gHSQC and gHMBC experiments.

${ }^{1} \mathrm{H}$ NMR $\left(400 \mathrm{MHz}, \mathrm{CDCl}_{3}, 25^{\circ} \mathrm{C}\right)$ :

${ }^{13} \mathrm{C} \mathrm{NMR}\left(100 \mathrm{MHz}, \mathrm{CDCl}_{3}, 25^{\circ} \mathrm{C}\right)$ :

FTIR (thin film) $\mathrm{cm}^{-1}$ :

HRMS (DART-TOF+) $m / z$ :

TLC (67\% EtOAc in hexanes), Rf:

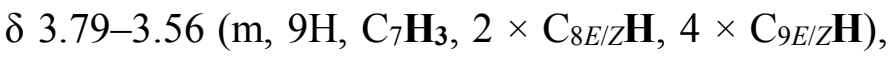
$3.48-3.38\left(\mathrm{~m}, 2 \mathrm{H}, 2 \times \mathrm{C}_{8 E / Z} \mathbf{H}\right), 3.31(\mathrm{t}, J=7.0 \mathrm{~Hz}$, $\left.1 \mathrm{H}, \mathrm{C}_{2} \mathbf{H}\right), 2.39-2.26\left(\mathrm{~m}, 2 \mathrm{H}, \mathrm{C}_{5} \mathbf{H}_{2}\right), 2.00-1.91(\mathrm{~m}$, $\left.4 \mathrm{H}, \mathrm{C}_{3} \mathbf{H}_{\mathbf{a}}, \mathrm{C}_{10} \mathbf{H}_{3}\right), 1.81-1.70\left(\mathrm{~m}, 2 \mathrm{H}, \mathrm{C}_{3} \mathbf{H}_{\mathbf{b}}, \mathrm{C}_{4} \mathbf{H}_{\mathbf{a}}\right)$, $1.65-1.56\left(\mathrm{~m}, 1 \mathrm{H}, \mathrm{C}_{4} \mathbf{H}_{\mathbf{b}}\right)$.

$\delta 173.8\left(\mathbf{C}_{\mathbf{6}}\right), 168.7\left(\mathbf{C}_{\mathbf{1}}\right), 67.1\left(\mathbf{C}_{\mathbf{9 E} / \mathbf{Z}}\right), 66.7\left(\mathbf{C}_{\mathbf{9 E} / \boldsymbol{Z}}\right)$, $51.6\left(\mathbf{C}_{7}\right), 46.4\left(\mathbf{C}_{\mathbf{8 E} / Z}\right), 42.4\left(\mathbf{C}_{\mathbf{8 E} / Z}\right), 41.9\left(\mathbf{C}_{2}\right), 33.7$ (C) $29.9\left(\mathbf{C}_{\mathbf{3}}\right), 22.8\left(\mathbf{C}_{4}\right), 11.2\left(\mathbf{C}_{\mathbf{1 0}}\right)$.

$2954(\mathrm{w}), 2921(\mathrm{w}), 2855(\mathrm{w}), 1731$ (s), 1633 (s), 1431 (s), 1248 (m), 1112 (s), 1024 (m), 847 (w), 570 (w).

$[\mathrm{M}+\mathrm{H}]^{+}$Calcd for $\mathrm{C}_{12} \mathrm{H}_{22} \mathrm{NO}_{4} \mathrm{~S}^{+}$276.1264;

Found 276.1263.

0.38 (UV, CAM). 


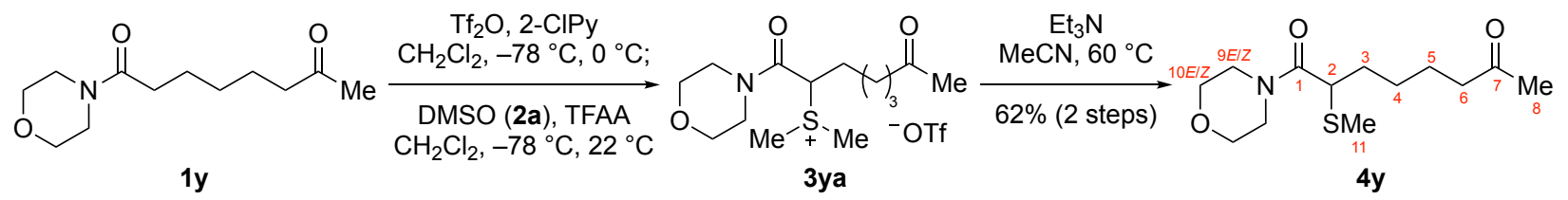

$\alpha$-SMe-Morpholine Amide 4y:

The $\alpha$-SMe-morpholine amide $\mathbf{4 y}$ was prepared via Method $\mathrm{C}$ using azeotropically dried morpholine amide 1y $(114 \mathrm{mg}, 500 \mu \mathrm{mol}, 1$ equiv) and dimethyl sulfoxide $(\mathbf{2 a}, 88.8 \mu \mathrm{L}$, $1.25 \mathrm{mmol}, 2.50$ equiv). The brown oily crude material was purified via flash column chromatography on silica gel (eluent: $50 \%$ EtOAc in hexanes) to yield $\alpha$-SMe-morpholine amide $4 \mathbf{y}(84.6 \mathrm{mg}, 62 \%)$ as an oil. Structural assignments were made using additional information from gCOSY, gHSQC and gHMBC experiments.

${ }^{1} \mathrm{H}$ NMR $\left(400 \mathrm{MHz}, \mathrm{CDCl}_{3}, 25^{\circ} \mathrm{C}\right)$ :

${ }^{13} \mathrm{C}$ NMR (100 MHz, $\left.\mathrm{CDCl}_{3}, 25{ }^{\circ} \mathrm{C}\right)$ :

FTIR (thin film) $\mathrm{cm}^{-1}$ :

HRMS (DART-TOF+) $m / z$ :

TLC (67\% EtOAc in hexanes), Rf: $\delta$ 3.79-3.56 (m, 6H, $\left.2 \times \mathrm{C}_{9 E / Z} \mathbf{H}, 4 \times \mathrm{C}_{10 E / Z} \mathbf{H}\right), 3.50$ $3.40\left(\mathrm{~m}, 2 \mathrm{H}, 2 \times \mathrm{C}_{9 E / Z} \mathbf{H}\right), 3.31(\mathrm{t}, J=7.2 \mathrm{~Hz}, 1 \mathrm{H}$, $\left.\mathrm{C}_{2} \mathbf{H}\right), 2.42\left(\mathrm{t}, J=7.3 \mathrm{~Hz}, 2 \mathrm{H}, \mathrm{C}_{6} \mathbf{H}_{2}\right), 2.11(\mathrm{~s}, 3 \mathrm{H}$, $\left.\mathrm{C}_{8} \mathbf{H}_{3}\right), 2.01\left(\mathrm{~s}, 3 \mathrm{H}, \mathrm{C}_{11} \mathbf{H}_{3}\right), 1.99-1.92\left(\mathrm{~m}, 1 \mathrm{H}, \mathrm{C}_{3} \mathbf{H}_{\mathbf{a}}\right)$, 1.75-1.66 (m, 1H, C $\left.\mathbf{H}_{\mathbf{b}}\right), 1.57$ (app-p, $J=7.5 \mathrm{~Hz}$, $\left.2 \mathrm{H}, \mathrm{C}_{5} \mathbf{H}_{2}\right), 1.44-1.23\left(\mathrm{~m}, 2 \mathrm{H}, \mathrm{C}_{4} \mathbf{H}_{2}\right)$.

$\delta 209.0\left(\mathbf{C}_{7}\right), 169.0\left(\mathbf{C}_{\mathbf{1}}\right), 67.1\left(\mathbf{C}_{\mathbf{1 0 E / Z}}\right), 66.8\left(\mathbf{C}_{\mathbf{1 0 E / Z}}\right)$, 46.5 ( $\left.\mathbf{C}_{9 E / Z}\right), 43.5\left(\mathbf{C}_{6}\right), 42.5\left(\mathbf{C}_{9 E / Z}\right), 41.9\left(\mathbf{C}_{2}\right), 30.3$ $\left(\mathbf{C}_{3}\right), 30.0\left(\mathbf{C}_{8}\right), 26.9\left(\mathbf{C}_{4}\right), 23.4\left(\mathbf{C}_{5}\right), 11.4\left(\mathbf{C}_{11}\right)$.

$2922(\mathrm{w}), 2856(\mathrm{w}), 1710(\mathrm{~m}), 1634(\mathrm{~s}), 1429(\mathrm{~m})$, 1359 (m), 1241 (m), 1112 (s), 1026 (m), 848 (w), 571 (w).

$[\mathrm{M}+\mathrm{H}]^{+}$Calcd for $\mathrm{C}_{13} \mathrm{H}_{24} \mathrm{NO}_{3} \mathrm{~S}^{+}$274.1471;

Found 274.1472.

0.23 (UV, CAM). 


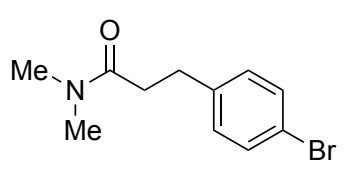

$1 z$

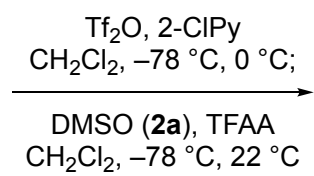

$\mathrm{CH}_{2} \mathrm{Cl}_{2},-78^{\circ} \mathrm{C}, 22^{\circ} \mathrm{C}$
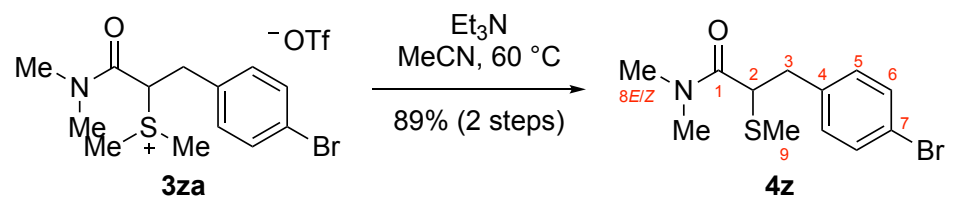

$\alpha$-SMe- $N, N$-Dimethyl Amide 4z:

The $\alpha$-SMe- $N, N$-dimethyl amide $4 \mathbf{z}$ was prepared via Method $\mathrm{C}$ using azeotropically dried $N, N$-dimethyl amide $\mathbf{1 z}(128 \mathrm{mg}, 500 \mu \mathrm{mol}, 1$ equiv) and dimethyl sulfoxide $(\mathbf{2 a}, 88.8 \mu \mathrm{L}$, $1.25 \mathrm{mmol}, 2.50$ equiv). The brown oily crude material was purified via flash column chromatography on silica gel (eluent: $20 \%$ EtOAc in hexanes) to yield $\alpha$-SMe- $N, N$-dimethyl amide $\mathbf{4 z}(134 \mathrm{mg}, 89 \%)$ as a solid. ${ }^{15}$ Structural assignments were made using additional information from gCOSY, gHSQC and gHMBC experiments.

${ }^{1} \mathrm{H} \mathrm{NMR}\left(400 \mathrm{MHz}, \mathrm{CDCl}_{3}, 25^{\circ} \mathrm{C}\right)$ :

${ }^{13} \mathrm{C} \mathrm{NMR}\left(100 \mathrm{MHz}, \mathrm{CDCl}_{3}, 25^{\circ} \mathrm{C}\right)$ :

FTIR (thin film) $\mathrm{cm}^{-1}$ :

HRMS (DART-TOF+) $m / z$ :

TLC (33\% EtOAc in hexanes), Rf:

M.p.: $\delta 7.38\left(\mathrm{~d}, J=8.0 \mathrm{~Hz}, 2 \mathrm{H}, 2 \times \mathrm{C}_{6} \mathbf{H}\right), 7.09(\mathrm{~d}, J=$ $\left.8.0 \mathrm{~Hz}, 2 \mathrm{H}, 2 \times \mathrm{C}_{5} \mathbf{H}\right), 3.57(\mathrm{dd}, J=8.6,6.0 \mathrm{~Hz}, 1 \mathrm{H}$, $\left.\mathrm{C}_{2} \mathbf{H}\right), 3.33\left(\mathrm{dd}, J=13.7,8.7 \mathrm{~Hz}, 1 \mathrm{H}, \mathrm{C}_{3} \mathbf{H}_{\mathbf{a}}\right), 2.94(\mathrm{~s}$, $\left.3 \mathrm{H}, \mathrm{C}_{8 E / Z} \mathbf{H}_{3}\right), 2.91-2.87\left(\mathrm{~m}, 4 \mathrm{H}, \mathrm{C}_{3} \mathbf{H}_{\mathbf{b}}, \mathrm{C}_{8 E / Z} \mathbf{H}_{3}\right), 2.10$ $\left(\mathrm{s}, 3 \mathrm{H}, \mathrm{C}_{9} \mathbf{H}_{3}\right)$.

$\delta 169.6\left(\mathbf{C}_{\mathbf{1}}\right), 138.2\left(\mathbf{C}_{4}\right), 131.5\left(\mathbf{C}_{6}\right), 131.1\left(\mathbf{C}_{5}\right)$, $120.5\left(\mathbf{C}_{7}\right), 44.1\left(\mathbf{C}_{2}\right), 37.4\left(\mathbf{C}_{8 E / Z}\right), 36.9\left(\mathbf{C}_{3}\right), 36.1$ $\left(\mathbf{C}_{8 E / Z}\right), 11.9\left(\mathbf{C}_{9}\right)$.

$2920(\mathrm{w}), 1631(\mathrm{~s}), 1487(\mathrm{~m}), 1396(\mathrm{~m}), 1258(\mathrm{w})$, $1139(\mathrm{~m}), 1072(\mathrm{~m}), 1009(\mathrm{~m}), 808(\mathrm{~s}), 665(\mathrm{w}), 528$ (m), 475 (m).

$[\mathrm{M}+\mathrm{H}]^{+}$Calcd for $\mathrm{C}_{12} \mathrm{H}_{17} \mathrm{BrNOS}^{+}$302.0209;

Found 302.0204.

0.28 (UV, CAM). $35-37{ }^{\circ} \mathrm{C}\left(\mathrm{CH}_{2} \mathrm{Cl}_{2}\right)$. 

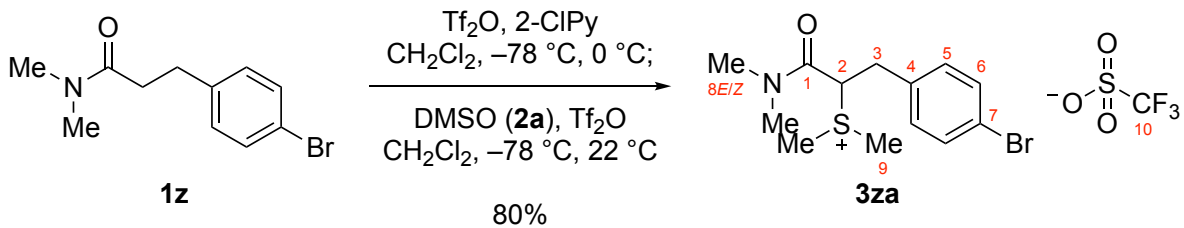

$\underline{N, N \text {-Dimethyl Amide Dimethyl Sulfonium Trifluoromethanesulfonate 3za: }}$

The $N, N$-dimethyl amide dimethyl sulfonium trifluoromethanesulfonate 3za was prepared via Method $\mathrm{C}$ without triethylamine treatment for demethylation using azeotropically dried $N, N$ dimethyl amide $\mathbf{1 z}(128 \mathrm{mg}, 500 \mu \mathrm{mol}, 1$ equiv) and dimethyl sulfoxide (2a, $88.8 \mu \mathrm{L}, 1.25 \mathrm{mmol}$, 2.50 equiv). After the addition of the dimethyl sulfoxide solution, trifluoromethanesulfonic anhydride $(84.1 \mu \mathrm{L}, 500 \mu \mathrm{mol}, 1.00$ equiv) was added, instead of trifluoroacetic anhydride. The brown oily crude material was purified via flash column chromatography on silica gel (eluent: EtOAc $\rightarrow 3 \%$ methanol in EtOAc) to yield $N, N$-dimethyl amide dimethyl sulfonium trifluoromethanesulfonate $\mathbf{3 z a}(187 \mathrm{mg}, 80 \%)$ as a solid that crystalized upon concentration. A single-crystal measuring $0.420 \times 0.385 \times 0.260 \mathrm{~mm}^{3}$ was selected, was mounted on a Bruker X8 Kappa DUO four-circle diffractometer equipped with a Bruker APEX2 CCD, and was analyzed using Mo K $\alpha$ radiation.

NMR-analysis of $N, N$-dimethyl amide dimethyl sulfonium trifluoromethanesulfonate 3za with 4-bromobenzotrifluoride as external NMR-standard revealed no exchange of the trifluoromethanesulfonate with bicarbonate in the aqueous work-up (sulfonium-cation: trifluoromethanesulfonate-anion $=1: 1 \quad(\mathrm{n} / \mathrm{n})) .{ }^{16} N, N$-Dimethyl amide dimethyl sulfonium trifluoromethanesulfonate 3za can be stored at $22{ }^{\circ} \mathrm{C}$ open to atmosphere for several weeks without any evidence of hydrolysis or elimination.

${ }^{1} \mathrm{H}$ NMR $\left(400 \mathrm{MHz}, \mathrm{CDCl}_{3}, 25^{\circ} \mathrm{C}\right): \quad 7.46\left(\mathrm{~d}, J=7.9 \mathrm{~Hz}, 2 \mathrm{H}, 2 \times \mathrm{C}_{6} \mathbf{H}\right), 7.16(\mathrm{~d}, J=8.0 \mathrm{~Hz}$, $\left.2 \mathrm{H}, 2 \times \mathrm{C}_{5} \mathbf{H}\right), 5.40\left(\mathrm{dd}, J=10.1,4.8 \mathrm{~Hz}, 1 \mathrm{H}, \mathrm{C}_{2} \mathbf{H}\right)$, $3.32\left(\mathrm{dd}, J=13.3,4.8 \mathrm{~Hz}, 1 \mathrm{H}, \mathrm{C}_{3} \mathbf{H}_{\mathbf{a}}\right), 3.19(\mathrm{dd}, J=$ $\left.13.2,10.0 \mathrm{~Hz}, 1 \mathrm{H}, \mathrm{C}_{3} \mathbf{H}_{\mathrm{b}}\right), 3.04$ (s, 3H, $\left.\mathrm{C}_{9} \mathbf{H}_{3}\right), 3.03$ (s, $\left.3 \mathrm{H}, \mathrm{C}_{9} \mathbf{H}_{3}\right), 2.86\left(\mathrm{~s}, 3 \mathrm{H}, \mathrm{C}_{8 E / Z} \mathbf{H}_{3}\right), 2.64(\mathrm{~s}, 3 \mathrm{H}$, $\left.\mathrm{C}_{8 E / Z} \mathbf{H}_{3}\right)$.

${ }^{13} \mathrm{C}$ NMR (100 MHz, $\left.\mathrm{CDCl}_{3}, 25{ }^{\circ} \mathrm{C}\right)$ :

${ }^{19} \mathrm{~F}$ NMR $\left(376 \mathrm{MHz}, \mathrm{CDCl}_{3}, 25{ }^{\circ} \mathrm{C}\right)$ :

FTIR (thin film) $\mathrm{cm}^{-1}$ :

HRMS (ESI-TOF+) $m / z$ : $\delta$ 165.1 $\left(\mathbf{C}_{\mathbf{1}}\right), 132.4\left(\mathbf{C}_{\mathbf{4}}, 2 \times \mathbf{C}_{\mathbf{6}}\right), 131.4\left(2 \times \mathbf{C}_{\mathbf{5}}\right), 122.6$ $\left(\mathbf{C}_{7}\right), 120.6\left(\mathrm{q}, J=320 \mathrm{~Hz}, \mathbf{C}_{\mathbf{1 0}}\right), 57.1\left(\mathbf{C}_{2}\right), 37.8$

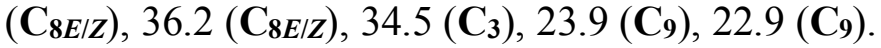

$\delta-79.4$.

3022 (w), 2937 (w), 1639 (m), 1489 (w), 1407 (m), 1249 (s), 1154 (s), 1027 (s), 810 (m), 755 (m), 636 (s), $516(\mathrm{~s})$.

$[\mathrm{M}]^{+}$Calcd for $\mathrm{C}_{13} \mathrm{H}_{19} \mathrm{BrNOS}^{+}$316.0365;

Found 316.0369 . 


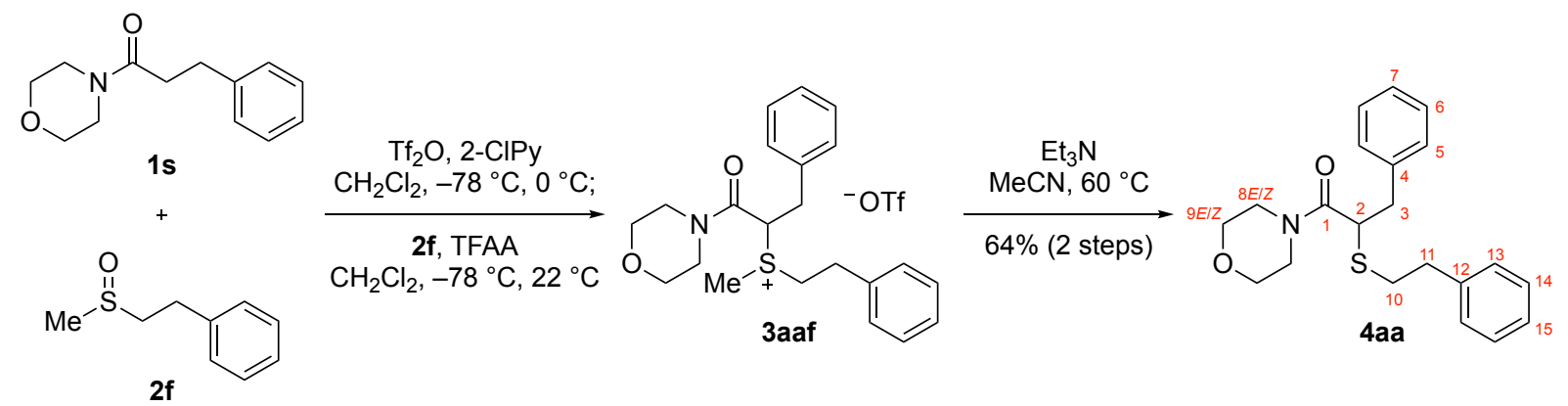

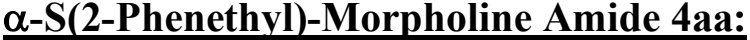

The $\alpha$-S(2-phenethyl)-morpholine amide 4aa was prepared via Method $\mathrm{C}$ using azeotropically dried morpholine amide $1 \mathrm{~s}(110 \mathrm{mg}, 500 \mu \mathrm{mol}, 1$ equiv) and methyl 2-phenethyl sulfoxide (2f, $210 \mathrm{mg}, 1.25 \mathrm{mmol}, 2.50$ equiv). The brown oily crude material was purified via flash column chromatography on silica gel (eluent: 20\% EtOAc in hexanes) to yield $\alpha-\mathrm{S}(2-$ phenethyl)-morpholine amide 4 aa (114 mg, 64\%) as a solid. ${ }^{15}$ Structural assignments were made using additional information from gCOSY, gHSQC and $\mathrm{gHMBC}$ experiments.

${ }^{1} \mathrm{H}$ NMR $\left(400 \mathrm{MHz}, \mathrm{CDCl}_{3}, 25^{\circ} \mathrm{C}\right)$ :

${ }^{13} \mathrm{C} \mathrm{NMR}\left(100 \mathrm{MHz}, \mathrm{CDCl}_{3}, 25^{\circ} \mathrm{C}\right)$ :

FTIR (thin film) $\mathrm{cm}^{-1}$ :

HRMS (DART-TOF+) $m / z$ :

TLC (33\% EtOAc in hexanes), Rf:

M.p.: $\delta$ 7.35-7.20 (m, 10H, $2 \times \mathrm{C}_{5} \mathbf{H}, 2 \times \mathrm{C}_{6} \mathbf{H}, \mathrm{C}_{7} \mathbf{H}, 2 \times$ $\left.\mathrm{C}_{13} \mathbf{H}, 2 \times \mathrm{C}_{14} \mathbf{H}, \mathrm{C}_{15} \mathbf{H}\right), 3.67(\mathrm{dd}, J=8.9,5.9 \mathrm{~Hz}, 1 \mathrm{H}$, $\left.\mathrm{C}_{2} \mathbf{H}\right), 3.64-3.48\left(\mathrm{~m}, 5 \mathrm{H}, 2 \times \mathrm{C}_{8 E / Z} \mathbf{H}, 3 \times \mathrm{C}_{9 E / Z} \mathbf{H}\right)$, 3.43-3.36 (m, 2H, $\left.\mathrm{C}_{3} \mathbf{H}_{\mathrm{a}}, \mathrm{C}_{8 E / Z} \mathbf{H}\right), 3.24-3.15(\mathrm{~m}, 2 \mathrm{H}$, $\left.\mathrm{C}_{8 E / Z} \mathbf{H}, \mathrm{C}_{9 E / Z} \mathbf{H}\right), 3.04(\mathrm{dd}, J=13.6,5.8 \mathrm{~Hz}, 1 \mathrm{H}$, $\mathrm{C}_{3} \mathbf{H}_{\mathbf{b}}$ ), 2.98-2.84 (m, 4H, $\left.\mathrm{C}_{10} \mathbf{H}_{2}, \mathrm{C}_{11} \mathbf{H}_{2}\right)$.

$\delta 169.1\left(\mathbf{C}_{\mathbf{1}}\right), 140.3\left(\mathbf{C}_{\mathbf{1 2}}\right), 138.9\left(\mathbf{C}_{4}\right), 129.3\left(2 \times \mathbf{C}_{5}\right)$, $128.7\left(2 \times \mathbf{C}_{\mathrm{Ar}}\right), 128.6\left(2 \times \mathbf{C}_{\mathrm{Ar}}\right), 128.6\left(2 \times \mathbf{C}_{\mathrm{Ar}}\right)$, $126.8\left(\mathbf{C}_{\mathbf{A r}}\right), 126.6\left(\mathbf{C}_{\mathbf{A r}}\right), 66.9\left(\mathbf{C}_{\mathbf{9 E} / \boldsymbol{Z}}\right), 66.5\left(\mathbf{C}_{\mathbf{9 E} / \boldsymbol{Z}}\right)$, $46.4\left(\mathbf{C}_{8 E / Z}\right), 44.6\left(\mathbf{C}_{2}\right), 42.5\left(\mathbf{C}_{8 E / Z}\right), 38.7\left(\mathbf{C}_{3}\right), 36.2$ $\left(\mathbf{C}_{11}\right), 31.2\left(\mathbf{C}_{\mathbf{1 0}}\right)$.

$3059(\mathrm{w}), 3025(\mathrm{w}), 2968(\mathrm{w}), 2929(\mathrm{w}), 2860(\mathrm{w})$, 1619 (s), $1492(\mathrm{~m}), 1450$ (s), $1225(\mathrm{~m}), 1108(\mathrm{~s}), 1022$ (m), $837(\mathrm{~m}), 746(\mathrm{~s}), 736(\mathrm{~s}), 698(\mathrm{~s}), 576(\mathrm{~m}), 517$ (m).

$[\mathrm{M}+\mathrm{H}]^{+}$Calcd for $\mathrm{C}_{21} \mathrm{H}_{26} \mathrm{NO}_{2} \mathrm{~S}^{+} 356.1679$;

Found 356.1682.

0.36 (UV, CAM).

$78-80{ }^{\circ} \mathrm{C}\left(\mathrm{CH}_{2} \mathrm{Cl}_{2}\right)$. 

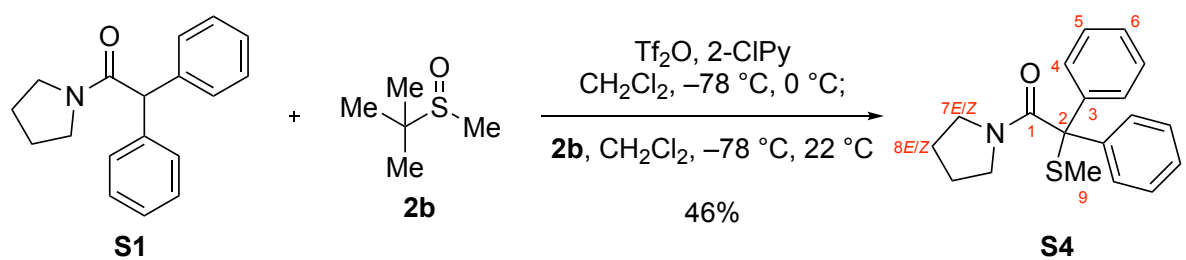

\section{$\alpha$-SMe-Pyrrolidine Amide S4: ${ }^{17}$}

The $\alpha$-SMe-pyrrolidine amide S4 was prepared via Method B using azeotropically dried pyrrolidine amide $\mathbf{S 1}$ (133 mg, $500 \mu \mathrm{mol}, 1$ equiv) and tert-butyl methyl sulfoxide $(\mathbf{2 b}, 69.8 \mu \mathrm{L}$, $600 \mu \mathrm{mol}, 1.20$ equiv). The bright orange oily crude material was purified via flash column chromatography on silica gel (eluent: $20 \%$ EtOAc in hexanes) to yield $\alpha$-SMe-pyrrolidine amide S4 (72.2 $\mathrm{mg}, 46 \%)$ as a solid. Structural assignments were made using additional information from gCOSY, gHSQC and gHMBC experiments.

${ }^{1} \mathrm{H}$ NMR $\left(400 \mathrm{MHz}, \mathrm{CDCl}_{3}, 25^{\circ} \mathrm{C}\right): \quad \delta 7.52-7.49\left(\mathrm{~m}, 4 \mathrm{H}, 4 \times \mathrm{C}_{4} \mathbf{H}\right), 7.35-7.31(\mathrm{~m}, 4 \mathrm{H}, 4 \times$ $\left.\mathrm{C}_{5} \mathbf{H}\right), 7.27-7.22\left(\mathrm{~m}, 2 \mathrm{H}, 2 \times \mathrm{C}_{6} \mathbf{H}\right), 3.62(\mathrm{t}, J=$ $\left.7.1 \mathrm{~Hz}, 2 \mathrm{H}, 2 \times \mathrm{C}_{7 E / Z} \mathbf{H}\right), 2.66(\mathrm{t}, J=6.6 \mathrm{~Hz}, 2 \mathrm{H}, 2 \times$ $\left.\mathrm{C}_{7 E / Z} \mathbf{H}\right), 1.90$ (s, 3H, $\left.\mathrm{C}_{9} \mathbf{H}_{3}\right), 1.71$ (app-p, $J=6.9 \mathrm{~Hz}$, $\left.2 \mathrm{H}, 2 \times \mathrm{C}_{8 E / Z} \mathbf{H}\right), 1.60$ (app-p, $J=6.5 \mathrm{~Hz}, 2 \mathrm{H}, 2 \times$ $\left.\mathrm{C}_{8 E / Z} \mathbf{H}\right)$.

${ }^{13} \mathrm{C}$ NMR $\left(100 \mathrm{MHz}, \mathrm{CDCl}_{3}, 25^{\circ} \mathrm{C}\right)$ :

FTIR (thin film) $\mathrm{cm}^{-1}$ :

HRMS (DART-TOF+) $m / z$ :

TLC (67\% EtOAc in hexanes), Rf:

M.p.: $\delta 169.7\left(\mathbf{C}_{1}\right), 139.7\left(2 \times \mathbf{C}_{3}\right), 129.0\left(4 \times \mathbf{C}_{4}\right), 128.4(4$ $\left.\times \mathbf{C}_{5}\right), 127.3\left(2 \times \mathbf{C}_{6}\right), 67.8\left(\mathbf{C}_{2}\right), 48.2\left(\mathbf{C}_{7 E / Z}\right), 48.1$ $\left(\mathbf{C}_{7 E / Z}\right), 26.7\left(\mathbf{C}_{8 E / Z}\right), 23.4\left(\mathbf{C}_{8 E / Z}\right), 15.8\left(\mathbf{C}_{9}\right)$.

2972 (w), 2914 (w), 2883 (w), $1621(\mathrm{~m}), 1394(\mathrm{~m})$, $1187(\mathrm{w}), 770(\mathrm{w}), 747(\mathrm{~m}), 702(\mathrm{~s}), 589(\mathrm{w}), 506(\mathrm{~m})$.

$[\mathrm{M}+\mathrm{H}]^{+}$Calcd for $\mathrm{C}_{19} \mathrm{H}_{22} \mathrm{NOS}^{+}$312.1417; Found 312.1411.

0.63 (UV, CAM). 93-95 ${ }^{\circ} \mathrm{C}\left(\mathrm{CH}_{2} \mathrm{Cl}_{2}\right)$. 


\section{Overview of In Situ IR Experiments:}

\section{In Situ IR Experiment A}<smiles>O=C(Cc1ccccc1)N1CCCC1</smiles>

$\underset{\mathrm{CH}_{2} \mathrm{Cl}_{2},-78{ }^{\circ} \mathrm{C}, 0{ }^{\circ} \mathrm{C}}{\stackrel{\mathrm{Tf}_{2} \mathrm{O}, 2-\mathrm{CIPy}}{\longrightarrow}}$

$1 b$

$1 b$

\section{In Situ IR Experiment B}

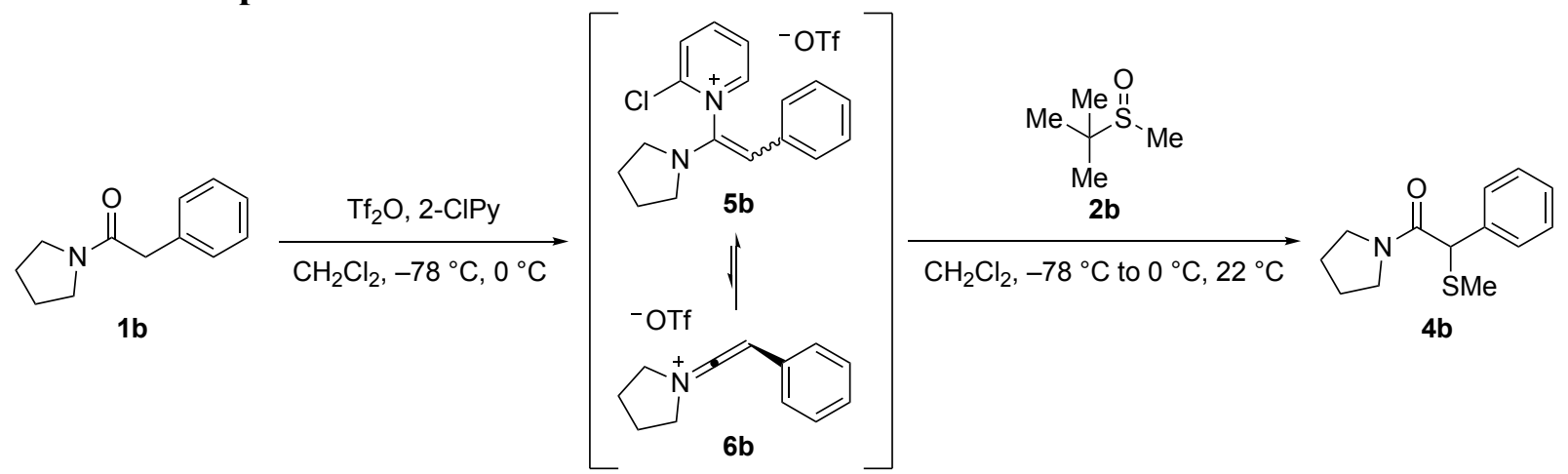<smiles>O=C(C=Cc1ccccc1)N1CCCC1</smiles>

\section{In Situ IR Experiment C}

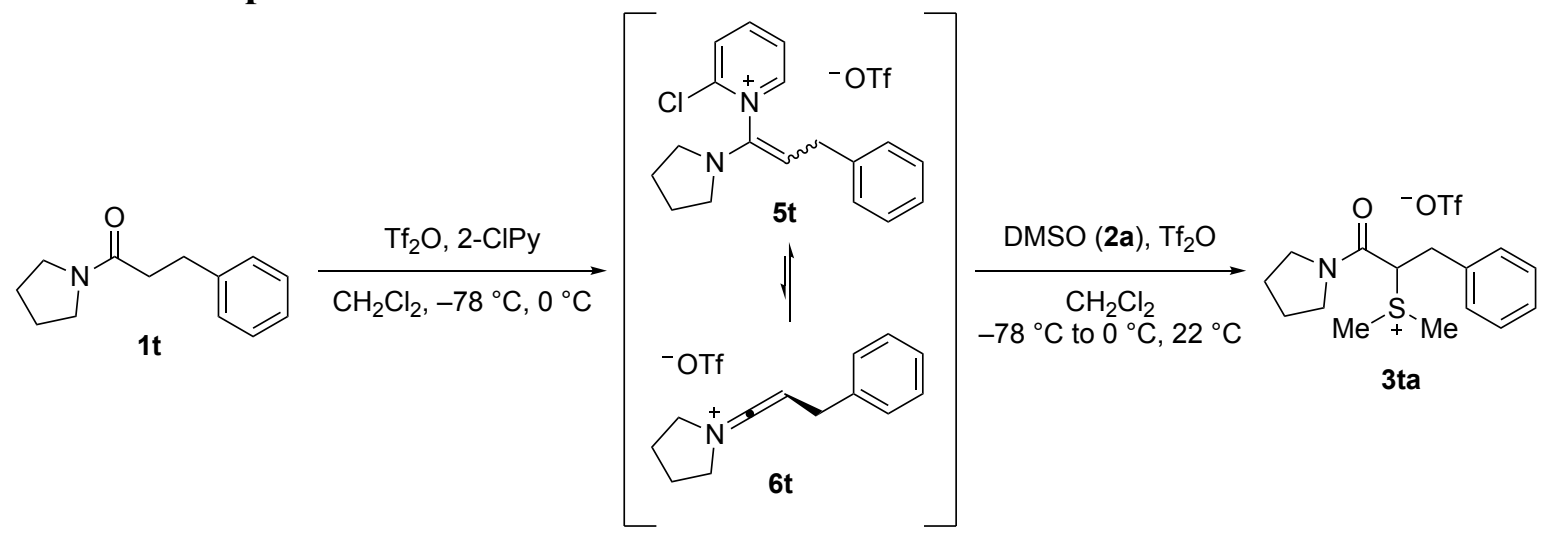


<smiles>O=C(Cc1ccccc1)N1CCCC1</smiles>

$1 \mathrm{~b}$<smiles></smiles>

\section{In Situ IR Experiment A. $\alpha$-Sulfidation of Pyrrolidine Amide 1b via Dimethyl Sulfoxide (2a)} Addition at $-78^{\circ} \mathrm{C}::^{18}$

Reactions were followed via in situ IR measurements in IR microcells under vigorous stirring $(500 \mathrm{rpm})$. Trifluoromethanesulfonic anhydride $(92.5 \mu \mathrm{L}, 550 \mu \mathrm{mol}, 1.10$ equiv) was added via syringe over $1 \mathrm{~min}$ to a stirred solution of pyrrolidine amide $\mathbf{1 b}(94.6 \mathrm{mg}, 500 \mu \mathrm{mol}$, 1 equiv) and 2-chloropyridine $(94.6 \mu \mathrm{L}, 1.00 \mathrm{mmol}, 2.00$ equiv) in dichloromethane $(1.7 \mathrm{~mL})$ at $-78{ }^{\circ} \mathrm{C}$ under argon atmosphere. The dark red solution was stirred at $0{ }^{\circ} \mathrm{C}$ for $15 \mathrm{~min}$, before it was again cooled to $-78^{\circ} \mathrm{C}$. A solution of dimethyl sulfoxide $(\mathbf{2 a}, 53.3 \mu \mathrm{L}, 750 \mu \mathrm{mol}, 1.50$ equiv) in dichloromethane $(0.3 \mathrm{~mL})$ was added at $-78^{\circ} \mathrm{C}$. The reaction mixture was allowed to warm slowly to $0{ }^{\circ} \mathrm{C}$ to give a yellow solution. Sulfoxide addition to the activated pyrrolidine amide $\mathbf{1 b}$ began at $-60{ }^{\circ} \mathrm{C}$ with full conversion when $-30^{\circ} \mathrm{C}$ was reached.

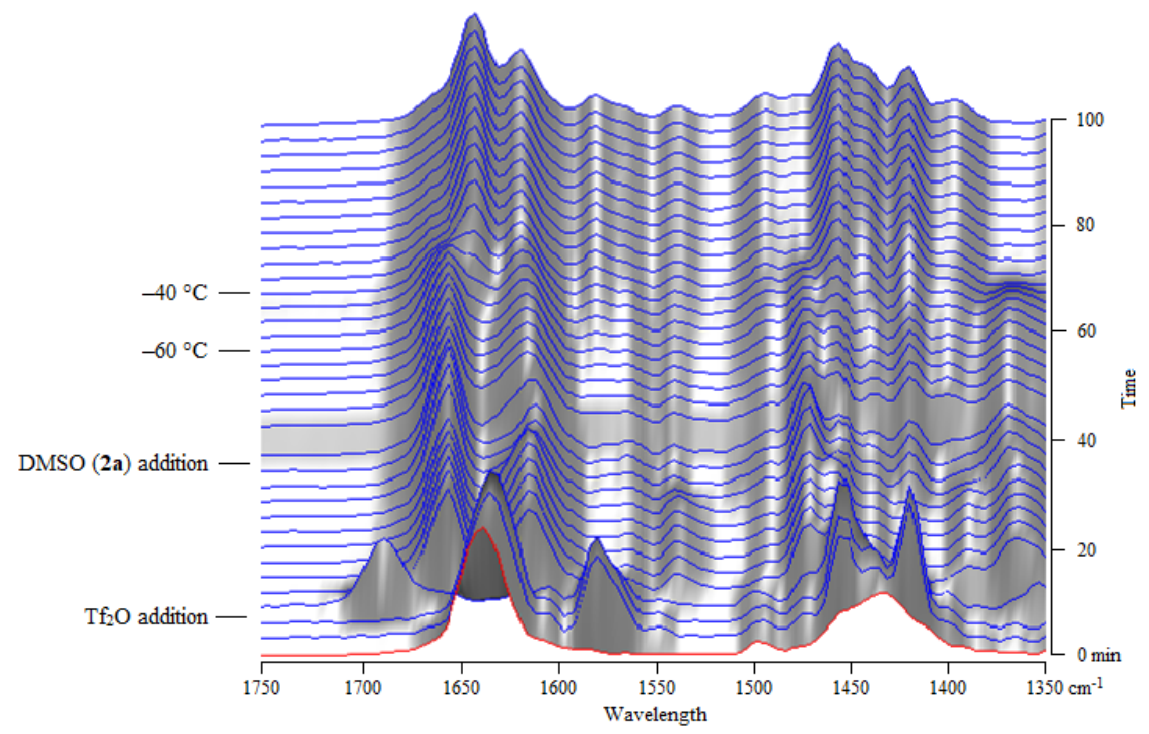

Figure S1: In situ IR spectrum from the pyrrolidine amide $\mathbf{1 b}$ activation and addition of DMSO (2a) at $-78^{\circ} \mathrm{C}$. Reaction followed over a period of $100 \mathrm{~min}$. IR spectra recorded after every $3 \mathrm{~min}$. Dichloromethane as background. 
<smiles>O=C(Cc1ccccc1)N1CCCC1</smiles>

$1 b$<smiles></smiles>

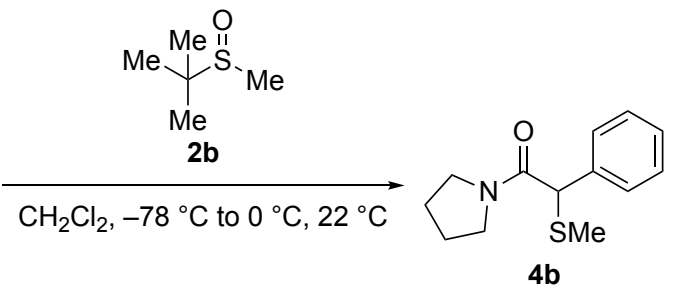

4b

\section{In Situ IR Experiment B. $\alpha$-Sulfidation of Pyrrolidine Amide $1 b$ via tert-Butyl Methyl} Sulfoxide (2b) Addition at $-78^{\circ} \mathrm{C}::^{18}$

This in situ IR experiment was conducted following the procedure described in Experiment A using pyrrolidine amide $1 \mathrm{~b}(94.6 \mathrm{mg}, 500 \mu \mathrm{mol}, 1$ equiv). After the dark red solution was cooled to $-78^{\circ} \mathrm{C}$, a solution of tert-butyl methyl sulfoxide $(2 \mathbf{b}, 87.3 \mu \mathrm{L}, 750 \mu$ mol, 1.50 equiv) in dichloromethane $(0.3 \mathrm{~mL})$ was added. The reaction mixture was allowed to warm slowly to $0{ }^{\circ} \mathrm{C}$ to give a yellow solution. Sulfoxide addition to the activated pyrrolidine amide $\mathbf{1 b}$ began at $-60{ }^{\circ} \mathrm{C}$ with full conversion when $-30{ }^{\circ} \mathrm{C}$ was reached. Prolonged reaction times at $0{ }^{\circ} \mathrm{C}$ as well as warming to $22{ }^{\circ} \mathrm{C}$ resulted in a decrease of the IR-band at $1648 \mathrm{~cm}^{-1}$.

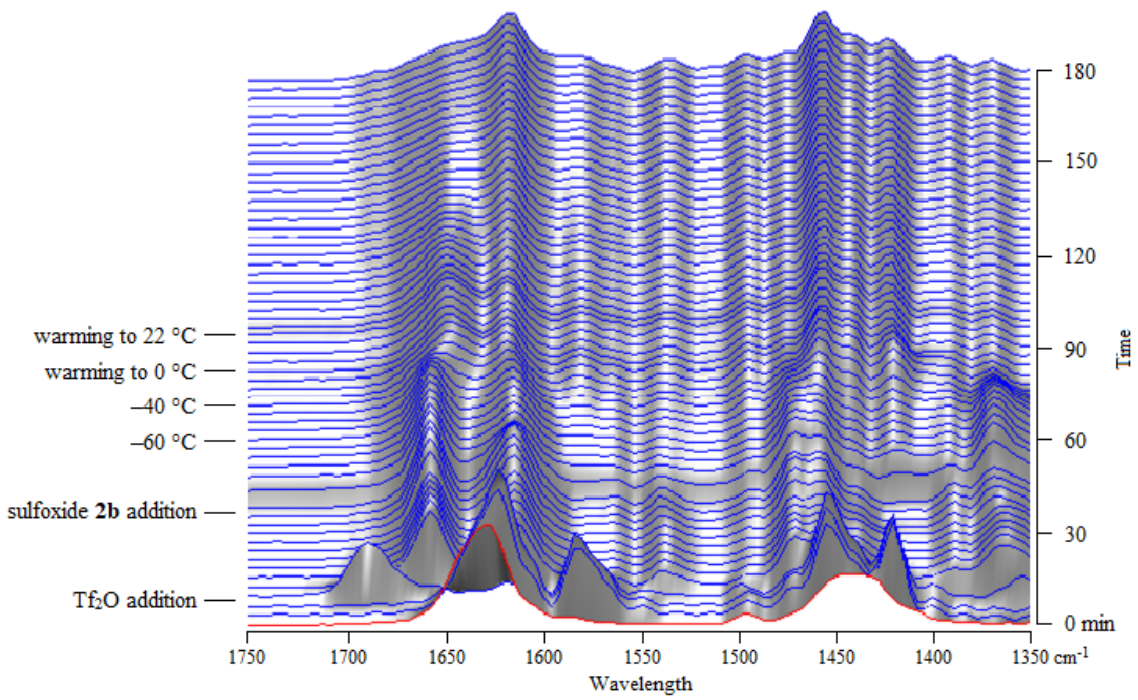

Figure S2: In situ IR spectrum from the pyrrolidine amide 1b activation and addition of tert-butyl methyl sulfoxide (2b) at $-78^{\circ} \mathrm{C}$. Reaction followed over a period of $180 \mathrm{~min}$. IR spectra recorded after every 3 min. Dichloromethane as background. 


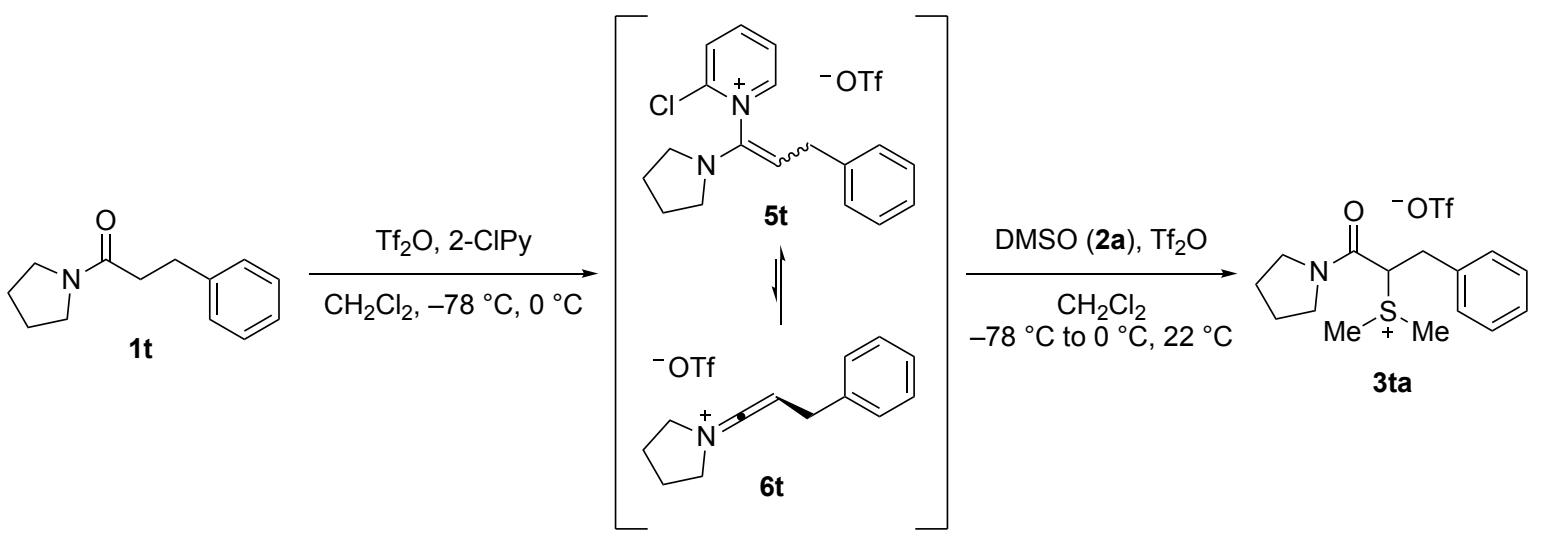

In Situ IR Experiment C. $\alpha$-Sulfidation of Pyrrolidine Amide 1t via Dimethyl Sulfoxide (2a) and Trifluoromethanesulfonic Anhydride Addition at $-78^{\circ} \mathrm{C}: \mathbf{:}^{18}$

This in situ IR experiment was conducted following the procedure described in Experiment A using pyrrolidine amide 1t $(102 \mathrm{mg}, 500 \mu \mathrm{mol}, 1$ equiv). A solution of dimethyl sulfoxide (2a, $88.8 \mu \mathrm{L}, 1.25 \mathrm{mmol}, 2.50$ equiv) in dichloromethane $(0.3 \mathrm{~mL})$ and trifluoromethanesulfonic anhydride $\left(84.1 \mu \mathrm{L}, 500 \mu \mathrm{mol}, 1.00\right.$ equiv) were added at $-78^{\circ} \mathrm{C}$. The yellow solution was stirred at $0{ }^{\circ} \mathrm{C}$ for $30 \mathrm{~min}$, then at $22^{\circ} \mathrm{C}$. Instant conversion of the activated amide was observed after sulfoxide addition at $-78^{\circ} \mathrm{C}$ and immediate warming to $0{ }^{\circ} \mathrm{C}$.

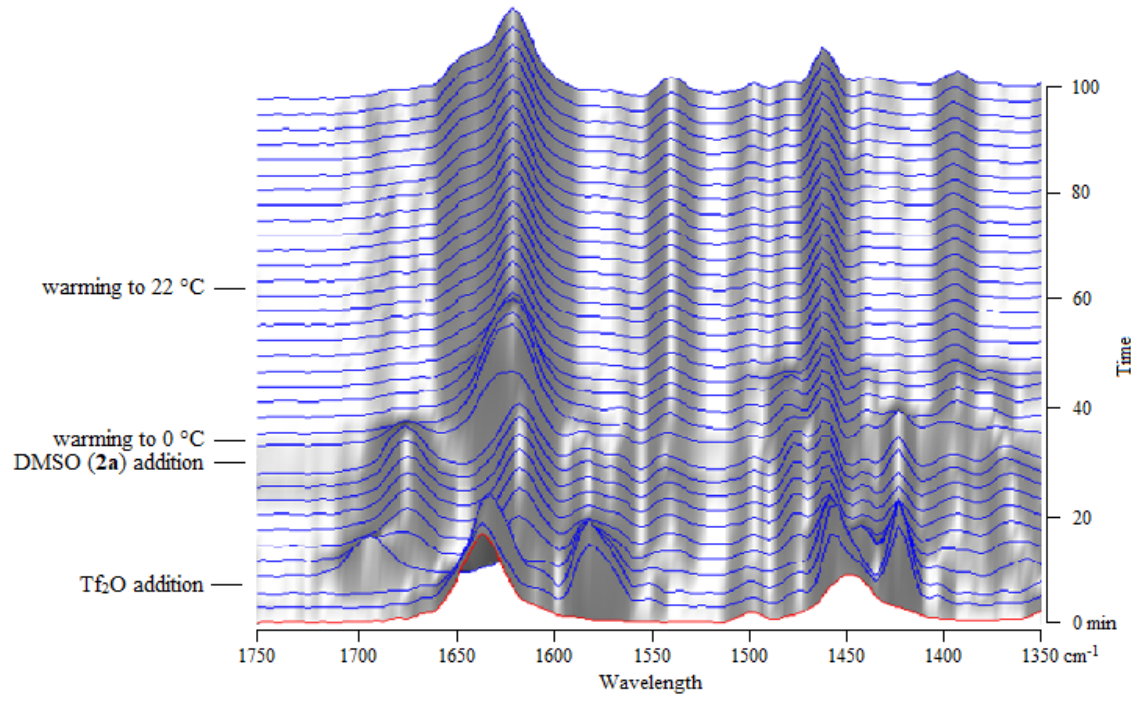

Figure S3: In situ IR spectrum from the pyrrolidine amide $\mathbf{1 t}$ activation as well as addition of $\mathrm{DMSO}(\mathbf{2 a})$ and $\mathrm{Tf}_{2} \mathrm{O}$ at $-78^{\circ} \mathrm{C}$. Reaction followed over a period of $100 \mathrm{~min}$. IR spectra recorded after every 3 min. Dichloromethane as background. 


\section{Overview of Mechanistic Experiments:}

\section{Mechanistic Experiment A}<smiles>O=C(Cc1ccccc1)N1CCCC1</smiles>

$1 b$

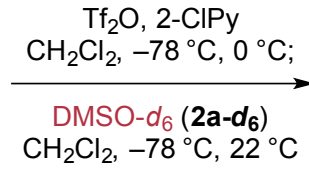

$\mathrm{CH}_{2} \mathrm{Cl}_{2},-78^{\circ} \mathrm{C}, 22^{\circ} \mathrm{C}$

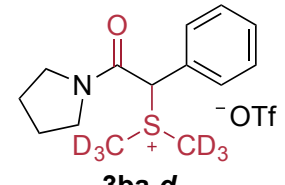

3 ba- $d_{6}$

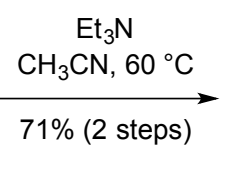

no incorporation at benzylic position

\section{Mechanistic Experiment B}<smiles>O=C(CCc1ccccc1)N1CCCC1</smiles>

$1 \mathrm{t}$

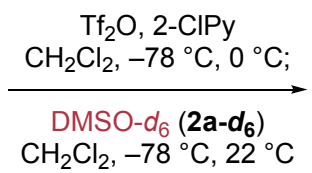

$85 \%$

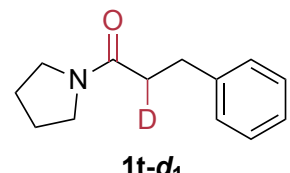

88 atom $\% D$ at $\alpha$-position

\section{Mechanistic Experiments C and D}

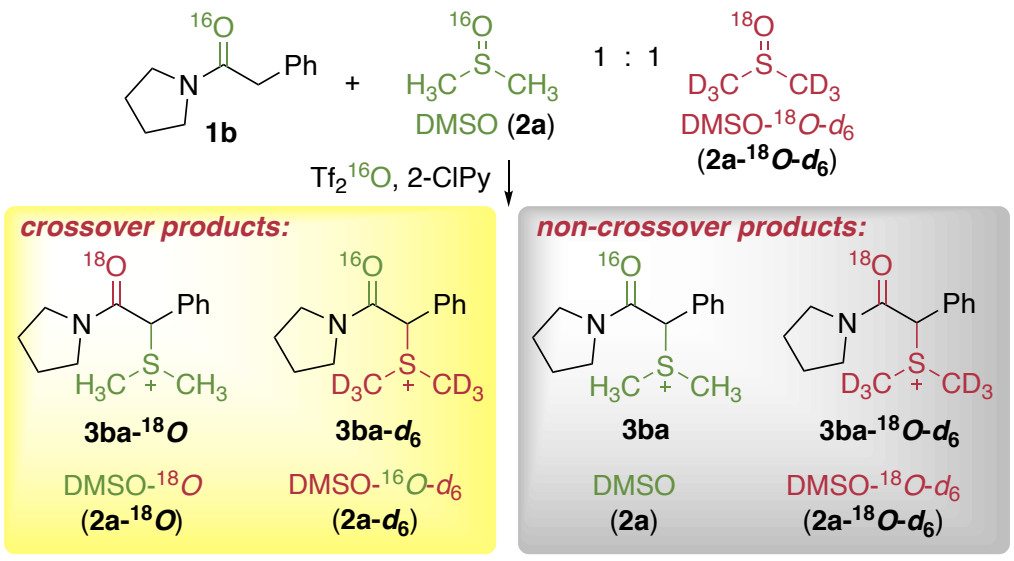

\section{Mechanistic Experiment E}<smiles>O=C(Cc1ccccc1)N1CCCC1</smiles>

$1 \mathrm{~b}$

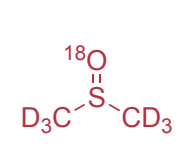

DMSO- ${ }^{18} \mathrm{O}-d_{6}\left(2 \mathrm{a}-{ }^{18} \mathrm{O}-\mathrm{d}_{6}\right)$

$$
93 \text { atom } \%{ }^{18} \mathrm{O}
$$

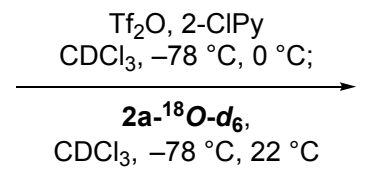

$\mathrm{DCl}_{3},-78^{\circ} \mathrm{C}, 22^{\circ} \mathrm{C}$<smiles>CS(=O)(=O)C(C(=O)N1CCCC1)c1ccccc1</smiles>

$\Delta m / z:+8$

$3 \mathrm{ba}-{ }^{18} \mathrm{O}-\mathrm{d}_{6}$

\section{Mechanistic Experiment F}<smiles>O=C(Cc1ccccc1)N1CCOCC1</smiles>

$1 a$
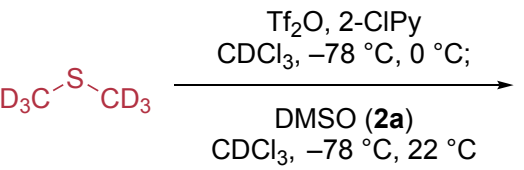

$\mathrm{CDCl}_{3},-78^{\circ} \mathrm{C}, 22^{\circ} \mathrm{C}$

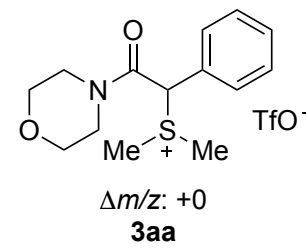<smiles>CC(C)(C)[SH](C(=O)[O-])C(C(=O)N1CCOCC1)c1ccccc1</smiles>

$\Delta m / z:+6$ 3 aa- $d_{6}$ 


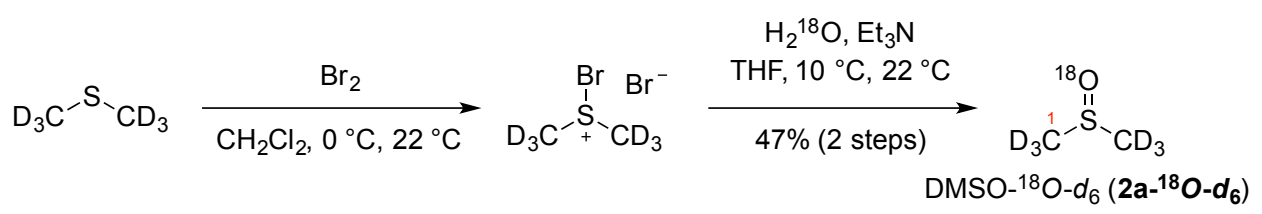

$\frac{\text { Dimethyl Sulfoxide- }{ }^{18} O-d_{6}}{\left(2 a-{ }^{18} O-d_{6}\right)}:^{19}$

A solution of bromine $(666 \mu \mathrm{L}, 13.0 \mathrm{mmol}, 1$ equiv) in anhydrous dichloromethane $(3.0 \mathrm{~mL})$ was added via syringe over $20 \mathrm{~min}$ to a solution of dimethyl sulfide- $d_{6}(1.05 \mathrm{~mL}$, $14.3 \mathrm{mmol}, 1.10$ equiv) in dichloromethane $(3.0 \mathrm{~mL})$ at $0{ }^{\circ} \mathrm{C}$ under argon atmosphere. The yellow suspension was stirred at $0{ }^{\circ} \mathrm{C}$ for $30 \mathrm{~min}$, then at $22{ }^{\circ} \mathrm{C}$ for another $30 \mathrm{~min}$. The yellow solid was filtered under intert atmosphere, and was washed with anhydrous diethyl ether $(3 \times 5 \mathrm{~mL})$. Drying under high vacuum gave bromodimethylsulfonium- $d_{6}$ bromide $(2.34 \mathrm{~g}, 79 \%)$ as a solid, which was used without further purification.

Bromodimethylsulfonium- $d_{6}$ bromide $(1.37 \mathrm{~g}, 6.00 \mathrm{mmol}, 1$ equiv) was added with a Teflon-coated spatula in five portions over $15 \mathrm{~min}$ to a solution of water- ${ }^{18} O(119 \mu \mathrm{L}, 6.60 \mathrm{mmol}$, 1.10 equiv) and triethylamine $(1.67 \mathrm{~mL}, 12.0 \mathrm{mmol}, 1.20$ equiv) in anhydrous tetrahydrofuran $(8.0 \mathrm{~mL})$ at $10{ }^{\circ} \mathrm{C}$. The resulting light yellow suspension was stirred at $22{ }^{\circ} \mathrm{C}$ for 30 min, was filtered, and the solids were washed with anhydrous diethyl ether $(3 \times 5 \mathrm{~mL})$. The filtrate was concentrated under reduced pressure, and the light brown crude material was purified by Kugelrohr distillation at $12-15$ Torr (oven temperature: $\left.130{ }^{\circ} \mathrm{C}\right)$ to yield dimethyl sulfoxide- ${ }^{18} \mathrm{O}-d_{6}\left(\mathbf{2 a}-{ }^{18} \boldsymbol{O}\right.$ $\boldsymbol{d}_{6}, 309 \mathrm{mg}, 60 \%, 93$ atom $\%{ }^{18} \mathrm{O}$ ) as an oil. The atom $\%{ }^{18} \mathrm{O}$ content was determined via Q-TOF analysis.

${ }^{13} \mathrm{C}$ NMR $\left(100 \mathrm{MHz}, \mathrm{CDCl}_{3}, 25^{\circ} \mathrm{C}\right): \quad \delta 39.5\left(\operatorname{app}-\operatorname{sept}(I=1), J=21.1 \mathrm{~Hz}, 2 \times \mathbf{C}_{1}\right)$.

FTIR (thin film) $\mathrm{cm}^{-1}$ :

HRMS (DART-TOF+) $m / z$ :
3403 (br), 1637 (m), 1021 (m), 971 (s), 823 (m), 760 (m).

$[\mathrm{M}+\mathrm{H}]^{+}$Calcd for $\mathrm{C}_{2} \mathrm{HD}_{6}{ }^{18} \mathrm{OS}^{+}$87.0631;

Found 87.0630. 
<smiles>O=C(Cc1ccccc1)N1CCCC1</smiles>

$1 b$

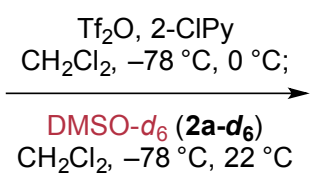

$\mathrm{CH}_{2} \mathrm{Cl}_{2},-78^{\circ} \mathrm{C}, 22^{\circ} \mathrm{C}$

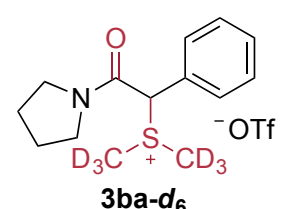

3 ba- $d_{6}$

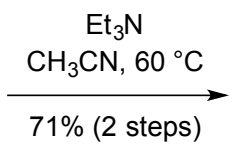

$4 \mathrm{~b}-d_{3}$<smiles>O=C(C(c1ccccc1)c1ccccc1)N1CCCC1</smiles>

no $D$ incorporation at benzylic position

Mechanistic Experiment A. Reaction of Pyrrolidine Amide 1b with Dimethyl Sulfoxide- $d_{6}$ $\left(2 \mathrm{a}-\mathbf{d}_{6}\right)$ :

Trifluoromethanesulfonic anhydride $(92.5 \mu \mathrm{L}, 550 \mu \mathrm{mol}, 1.05$ equiv) was added via syringe over $1 \mathrm{~min}$ to a stirred solution of pyrrolidine amide $\mathbf{1 b}(94.6 \mathrm{mg}, 500 \mu \mathrm{mol}, 1$ equiv) and 2-chloropyridine $(94.6 \mu \mathrm{L}, 1.00 \mathrm{mmol}, 2.00$ equiv) in anhydrous dichloromethane $(1.7 \mathrm{~mL})$ at $-78^{\circ} \mathrm{C}$ under argon atmosphere. The dark red solution was stirred at $0{ }^{\circ} \mathrm{C}$ for $15 \mathrm{~min}$, before it was again cooled to $-78{ }^{\circ} \mathrm{C}$. A solution of dimethyl sulfoxide- $d_{6}\left(\mathbf{2 a -} \boldsymbol{d}_{\mathbf{6}}, 53.0 \mu \mathrm{L}, 750 \mu \mathrm{mol}, 1.50\right.$ equiv) in dichloromethane $(0.3 \mathrm{~mL})$ was added via cannula over $2 \mathrm{~min}$ at $-78^{\circ} \mathrm{C}$. The reaction mixture was stirred at $0{ }^{\circ} \mathrm{C}$ for $15 \mathrm{~min}$, then at $22^{\circ} \mathrm{C}$ for $30 \mathrm{~min}$ to give a yellow solution, which was concentrated under reduced pressure. NMR-analysis of pyrrolidine amide sulfonium- $d_{6}$ trifluoromethanesulfonate $\mathbf{3} \mathbf{b a}-\boldsymbol{d}_{\mathbf{6}}$ did not show deuterium incorporation at the $\alpha$-position. The orange oily residue was dissolved in acetonitrile $(2.0 \mathrm{~mL})$. Triethylamine $(697 \mu \mathrm{L}, 5.00 \mathrm{mmol}$, 10.0 equiv) was added, and the resulting brown solution was stirred in a sealed roundbottom flask at $60{ }^{\circ} \mathrm{C}$ for $15 \mathrm{~h}$. After concentration under reduced pressure, the brown oily crude material was purified via flash column chromatography on silica gel (eluent: $25 \%$ EtOAc in hexanes) to yield $\alpha$-SMe-pyrrolidine- $d_{3}$ amide $\mathbf{4 b - d _ { 3 }}(85.0 \mathrm{mg}, 71 \%)$ as a solid.

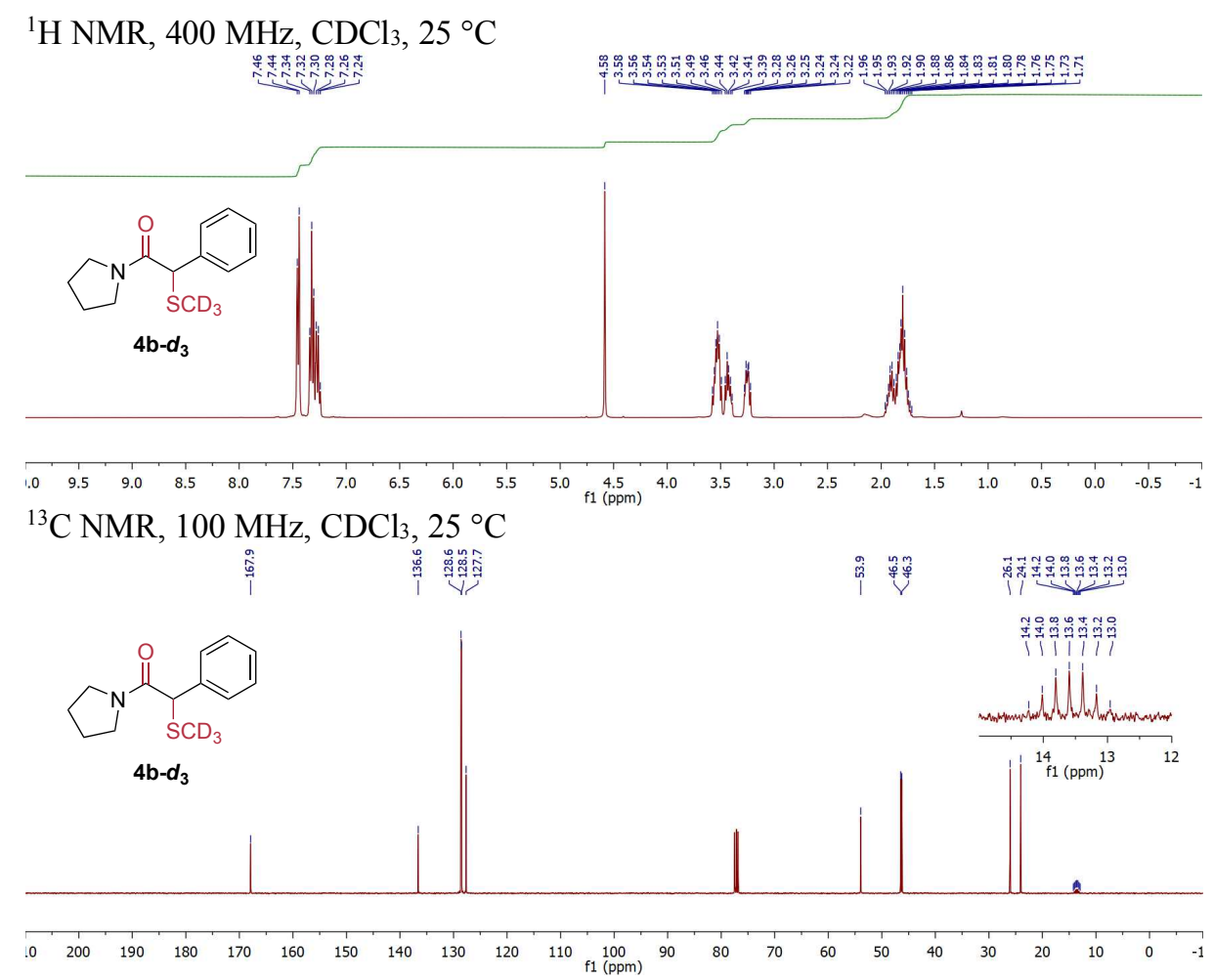

Figure S4: ${ }^{1} \mathrm{H}$ NMR-spectrum (above) and ${ }^{13} \mathrm{C}$ NMR-spectrum (below) of $\alpha$-SMe-pyrrolidine- $d_{3}$ amide $\mathbf{4 b}$ - $\boldsymbol{d}_{\mathbf{3}}$ from the reaction of pyrrolidine amide $\mathbf{1 b}$ with DMSO- $d_{6}\left(\mathbf{2} \mathbf{a}-\boldsymbol{d}_{\mathbf{6}}\right)$. 


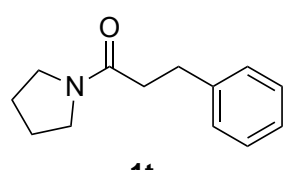

1t

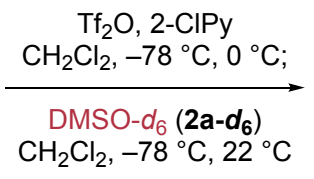

$85 \%$

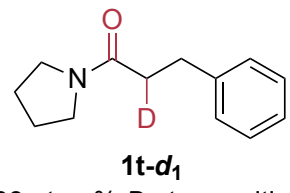

88 atom $\%$ at $\alpha$-position

Mechanistic Experiment B. Reaction of Pyrrolidine Amide 1t with Dimethyl Sulfoxide- $d_{6}$ (2a$\underline{\left.d_{6}\right):}$

Trifluoromethanesulfonic anhydride $(92.5 \mu \mathrm{L}, 550 \mu \mathrm{mol}, 1.05$ equiv) was added via syringe over $1 \mathrm{~min}$ to a stirred solution of pyrrolidine amide $1 \mathrm{t}$ (102 $\mathrm{mg}, 500 \mu \mathrm{mol}, 1$ equiv) and 2-chloropyridine $(94.6 \mu \mathrm{L}, 1.00 \mathrm{mmol}, 2.00$ equiv) in anhydrous dichloromethane $(1.7 \mathrm{~mL})$ at $-78^{\circ} \mathrm{C}$ under argon atmosphere. The red solution was stirred at $0{ }^{\circ} \mathrm{C}$ for $15 \mathrm{~min}$, before it was again cooled to $-78^{\circ} \mathrm{C}$. A solution of dimethyl sulfoxide- $d_{6}\left(\mathbf{2 a}-d_{6}, 53.0 \mu \mathrm{L}, 750 \mu \mathrm{mol}, 1.50\right.$ equiv $)$ in dichloromethane $(0.3 \mathrm{~mL})$ was added via cannula over $2 \mathrm{~min}$ at $-78^{\circ} \mathrm{C}$. The reaction mixture was stirred at $0{ }^{\circ} \mathrm{C}$ for $15 \mathrm{~min}$, then at $22{ }^{\circ} \mathrm{C}$ for $30 \mathrm{~min}$ to give a yellow solution. ${ }^{20} \mathrm{~A}$ saturated aqueous solution of sodium bicarbonate $(2.0 \mathrm{~mL})$ followed by dichloromethane $(1.0 \mathrm{~mL})$ was added, and the yellow biphasic mixture was vigorously stirred at $22{ }^{\circ} \mathrm{C}$ for $15 \mathrm{~min}$. After separation of the organic layer, the aqueous phase was extracted with dichloromethane $(3 \times 1.0 \mathrm{~mL})$. The combined light yellow organic extracts were dried over sodium sulfate, were filtered, and were concentrated under reduced pressure. The brown oily crude material was purified via flash column chromatography on silica gel (eluent: $33 \%$ EtOAc in hexanes) to yield pyrrolidine amide 1t- $\boldsymbol{d}_{\mathbf{1}}$ $(87.0 \mathrm{mg}, 85 \%)$ as an oil. Deuterium incorporation was determined to 88 atom $\% D$ by NMRanalysis and Q-TOF spectrometry.

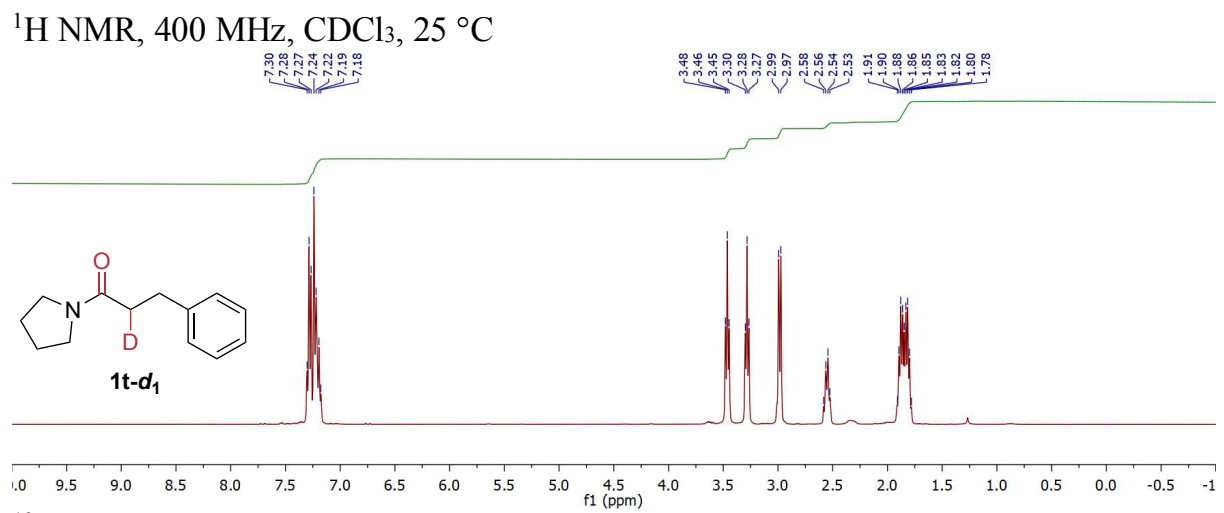

${ }^{13} \mathrm{C} \mathrm{NMR,} 100 \mathrm{MHz}, \mathrm{CDCl}_{3}, 25^{\circ} \mathrm{C}$

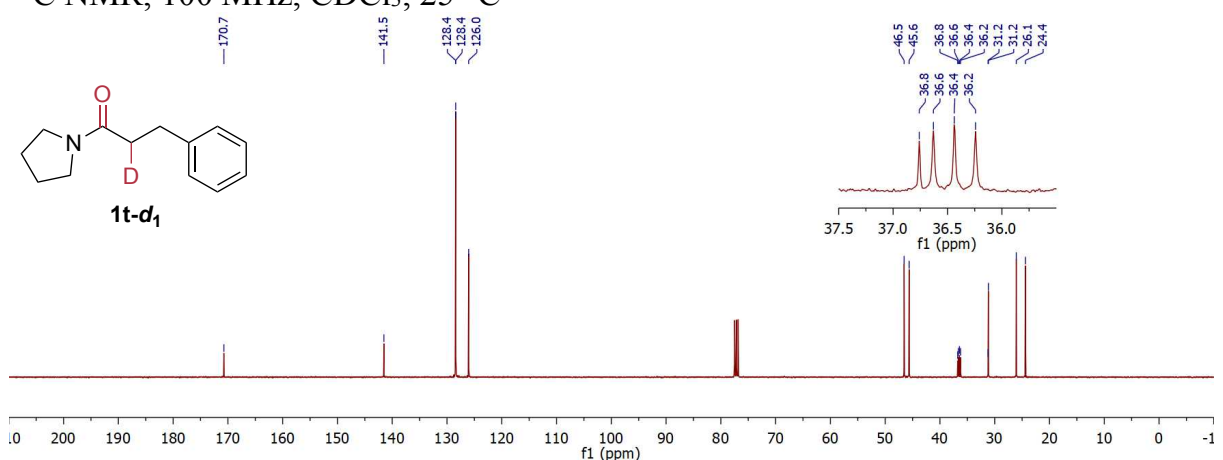

Figure S5: ${ }^{1} \mathrm{H}$ NMR-spectrum (above) and ${ }^{13} \mathrm{C}$ NMR-spectrum (below) of pyrrolidine amide- $d \mathbf{1 t}-\boldsymbol{d}_{\mathbf{1}}$ from the reaction of pyrrolidine amide $\mathbf{1 t}$ with DMSO- $d_{6}\left(\mathbf{2} \mathbf{a}-\boldsymbol{d}_{\mathbf{6}}\right)$. 


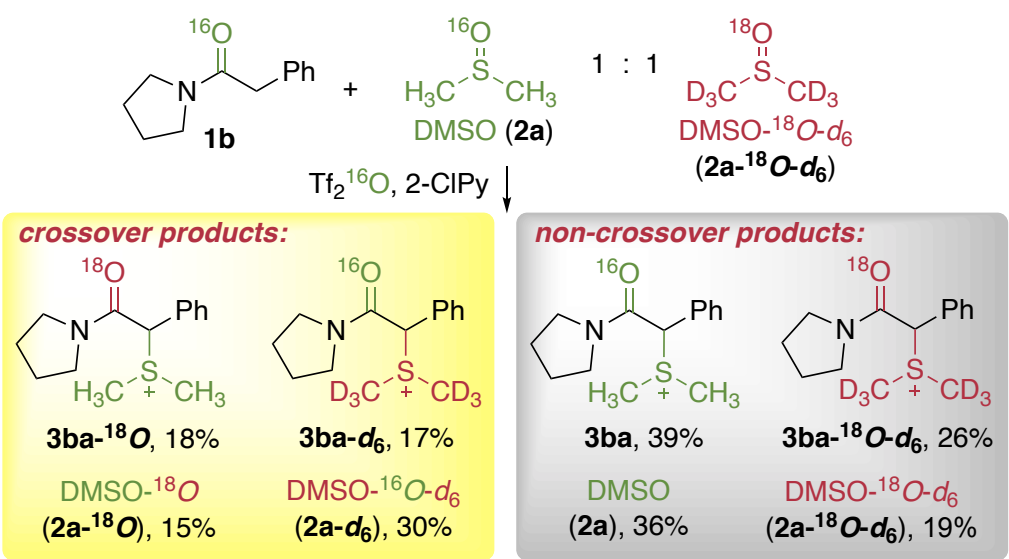

Mechanistic Experiment C. Crossover Experiment in the $\alpha$-Sulfidation of Pyrrolidine Amide 1b with a 1:1 Mixture of Dimethyl Sulfoxide (2a) and Dimethyl Sulfoxide- ${ }^{18} O-d_{6}\left(2 a-{ }^{18} O-d_{6}\right):$

Trifluoromethanesulfonic anhydride $(18.5 \mu \mathrm{L}, 110 \mu \mathrm{mol}, 1.10$ equiv) was added via syringe over $1 \mathrm{~min}$ to a stirred solution of pyrrolidine amide $\mathbf{1 b}(18.9 \mathrm{mg}, 100 \mu \mathrm{mol}, 1 \mathrm{equiv})$ and 2-chloropyridine $\left(18.9 \mu \mathrm{L}, 200 \mu \mathrm{mol}, 2.00\right.$ equiv) in chloroform- $d(320 \mu \mathrm{L})$ at $-78{ }^{\circ} \mathrm{C}$ under argon atmosphere. The dark red solution was stirred at $0{ }^{\circ} \mathrm{C}$ for $15 \mathrm{~min}$, before it was again cooled to $-78^{\circ} \mathrm{C}$. A solution of anhydrous dimethyl sulfoxide (2a, $7.1 \mu \mathrm{L}, 100 \mu \mathrm{mol}, 1.0$ equiv) and dimethyl sulfoxide- ${ }^{18} O-d_{6}\left(\mathbf{2 a}-{ }^{18} \boldsymbol{O}-\boldsymbol{d}_{\mathbf{6}}, 8.6 \mathrm{mg}, 100 \mu \mathrm{mol}, 1.0\right.$ equiv $)$ in anhydrous chloroform- $d(80 \mu \mathrm{L})$ was added via cannula over $2 \mathrm{~min}$ at $-78^{\circ} \mathrm{C}$. The reaction mixture was stirred at $0{ }^{\circ} \mathrm{C}$ for $15 \mathrm{~min}$, then at $22{ }^{\circ} \mathrm{C}$ for $30 \mathrm{~min}$ to give a yellow solution. Concentration under reduced pressure $\left(30^{\circ} \mathrm{C}\right.$, 100 Torr, $10 \mathrm{~min}$ ) gave a yellow crude material, which was analyzed via Q-TOF mass spectrometry. Substantial formation of crossover products is consistent with an intermolecular mechanism.

Table S4: Crossover experiment in the $\alpha$-sulfidation of pyrrolidine amide $1 \mathbf{b}$ with a $1: 1$ mixture of DMSO (2a):DMSO- ${ }^{18} O-d_{6}\left(\mathbf{2 a}-{ }^{18} \boldsymbol{O}-\boldsymbol{d}_{\mathbf{6}}\right)$ and $\mathrm{Tf}_{2} \mathrm{O}$ (1.10 equiv).

\begin{tabular}{ccccccc}
\hline & & $\begin{array}{c}\text { DMSO mix. } \\
\text { before }\end{array}$ & $\begin{array}{c}\text { DMSO mix. } \\
\text { after }\end{array}$ & $\begin{array}{c}\text { Triflate } \\
\text { before }^{[a]}\end{array}$ & $\begin{array}{c}\text { Triflate } \\
\text { after }\end{array}$ & Product \\
\hline$\Delta \boldsymbol{m} / z:$ & $+\mathbf{0}$ & $48 \%$ & $36 \%$ & $96 \%$ & $92 \%$ & $39 \%$ \\
& $+\mathbf{+ 2}$ & $4 \%$ & $15 \%$ & $4 \%$ & $8 \%$ & $18 \%$ \\
& $+\mathbf{6}$ & $4 \%$ & $30 \%$ & - & - & $17 \%$ \\
$+\mathbf{+ 8}$ & $44 \%$ & $19 \%$ & - & - & $26 \%$ \\
\hline
\end{tabular}

${ }^{[a]}$ Determined from a control experiment without labelled compounds. 


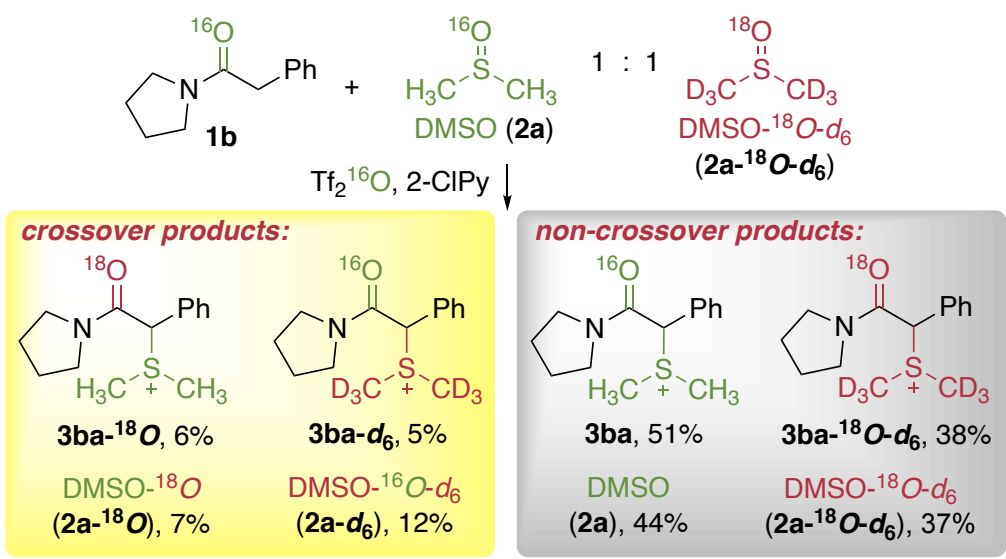

Mechanistic Experiment D. Crossover Experiment in the $\alpha$-Sulfidation of Pyrrolidine Amide 1b with a 1:1 Mixture of Dimethyl Sulfoxide (2a) and Dimethyl Sulfoxide- ${ }^{18} O-d_{6}\left(2 a_{-}{ }^{18} O-d_{6}\right)$ Using Substoichiometric Trifluoromethanesulfonic Anhydride:

The crossover experiment was executed following the representative procedure from Mechanistic Experiment C using pyrrolidine amide 1b (18.9 mg, $100 \mu \mathrm{mol}, 1$ equiv), trifluoromethanesulfonic anhydride $(8.4 \mu \mathrm{L}, 50 \mu \mathrm{mol}, 0.50$ equiv $)$, dimethyl sulfoxide $(\mathbf{2 a}, 7.1 \mu \mathrm{L}$, $100 \mu \mathrm{mol}, 1.0$ equiv) and dimethyl sulfoxide- ${ }^{18} \mathrm{O}-d_{6}\left(\mathbf{2 a -}{ }^{\mathbf{1 8}} \boldsymbol{O}-\boldsymbol{d}_{\mathbf{6}}, 8.6 \mathrm{mg}, 100 \mu \mathrm{mol}, 1.0\right.$ equiv). Concentration under reduced pressure $\left(30^{\circ} \mathrm{C}, 100\right.$ Torr, $\left.10 \mathrm{~min}\right)$ gave a yellow crude material, which was analyzed via Q-TOF mass spectrometry. The proportion of crossover products is diminished when a substoichiometric quantity of trifluoromethanesulfonic anhydride in used in the crossover experiment, as compared to a slight excess in the standard conditions (page S62). This observation is consistent with the role of electrophilically-activated sulfoxides in promoting an intermolecular sulfidation reaction.

Table S5: Crossover experiment in the $\alpha$-sulfidation of pyrrolidine amide $\mathbf{1 b}$ with a 1:1 mixture of DMSO (2a):DMSO- ${ }^{18} O-d_{6}\left(\mathbf{2 a}-{ }^{\mathbf{1 8}} \boldsymbol{O}-\boldsymbol{d}_{\mathbf{6}}\right)$ and $\mathrm{Tf}_{2} \mathrm{O}(0.50$ equiv).

\begin{tabular}{ccccccc}
\hline & & $\begin{array}{c}\text { DMSO mix. } \\
\text { before }\end{array}$ & $\begin{array}{c}\text { DMSO mix. } \\
\text { after }\end{array}$ & $\begin{array}{c}\text { Triflate } \\
\text { before }^{[a]}\end{array}$ & $\begin{array}{c}\text { Triflate } \\
\text { after }\end{array}$ & Product \\
\hline$\Delta \boldsymbol{m} / \boldsymbol{z}:$ & $+\mathbf{0}$ & $47 \%$ & $44 \%$ & $96 \%$ & $94 \%$ & $51 \%$ \\
& $+\mathbf{2}$ & $3 \%$ & $7 \%$ & $4 \%$ & $6 \%$ & $6 \%$ \\
& +6 & $4 \%$ & $12 \%$ & - & - & $5 \%$ \\
$+\mathbf{+ 8}$ & $46 \%$ & $37 \%$ & - & - & $38 \%$ \\
\hline
\end{tabular}

${ }^{[a]}$ Determined from a control experiment without labelled compounds. 


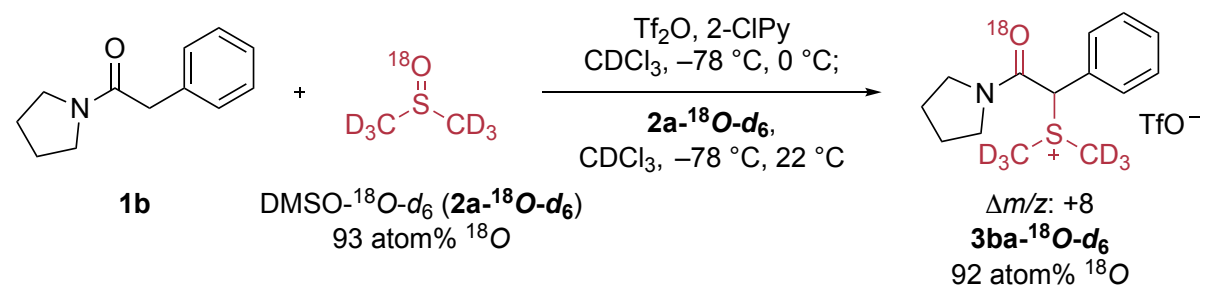

Mechanistic Experiment E. $\alpha$-Sulfidation of Pyrrolidine Amide 1b with Dimethyl Sulfoxide${ }^{18} O-d_{6}\left(2 \mathrm{a}^{18} \mathrm{O}-\mathrm{d}_{6}\right)$ :

Trifluoromethanesulfonic anhydride $(18.5 \mu \mathrm{L}, 110 \mu \mathrm{mol}, 1.10$ equiv) was added via syringe over $1 \mathrm{~min}$ to a stirred solution of pyrrolidine amide $\mathbf{1 b}(18.9 \mathrm{mg}, 100 \mu \mathrm{mol}, 1$ equiv) and 2-chloropyridine ( $18.9 \mu \mathrm{L}, 200 \mu \mathrm{mol}, 2.00$ equiv) in chloroform- $d(320 \mu \mathrm{L})$ at $-78{ }^{\circ} \mathrm{C}$ under argon atmosphere. The dark red solution was stirred at $0{ }^{\circ} \mathrm{C}$ for $15 \mathrm{~min}$, before it was again cooled to $-78{ }^{\circ} \mathrm{C}$. A solution of dimethyl sulfoxide- ${ }^{18} O-d_{6}\left(\mathbf{2 a}^{18}{ }^{18} \boldsymbol{O}-\boldsymbol{d}_{\mathbf{6}}, 17.2 \mathrm{mg}, 200 \mu \mathrm{mol}, 2.00\right.$ equiv) in chloroform- $d(80 \mu \mathrm{L})$ was added via cannula over $2 \mathrm{~min}$ at $-78^{\circ} \mathrm{C}$. The reaction mixture was stirred at $0{ }^{\circ} \mathrm{C}$ for $15 \mathrm{~min}$, then at $22^{\circ} \mathrm{C}$ for $30 \mathrm{~min}$ to give a yellow solution. Concentration under reduced pressure $\left(30^{\circ} \mathrm{C}, 100\right.$ Torr, $\left.10 \mathrm{~min}\right)$ gave a yellow crude material, which was analyzed via Q-TOF mass spectrometry.

Table S6: $\alpha$-Sulfidation of pyrrolidine amide $\mathbf{1 b}$ with DMSO- ${ }^{18} O-d_{6}\left(\mathbf{2 a}-{ }^{18} \boldsymbol{O}-\boldsymbol{d}_{\mathbf{6}}\right)$ and $\mathrm{Tf}_{2} \mathrm{O}$ (1.10 equiv).

\begin{tabular}{|c|c|c|c|c|c|c|}
\hline & & $\begin{array}{c}\text { DMSO- }-{ }^{18} O-d_{6} \\
\text { before }\end{array}$ & $\begin{array}{c}\text { DMSO- }{ }^{18} O-d_{6} \\
\text { after }\end{array}$ & $\begin{array}{l}\text { Triflate } \\
\text { before }^{[a]}\end{array}$ & $\begin{array}{c}\text { Triflate } \\
\text { after }\end{array}$ & Product \\
\hline \multirow[t]{4}{*}{$\Delta m / z:$} & $+\mathbf{0}$ & - & - & $96 \%$ & $93 \%$ & - \\
\hline & +2 & - & - & $4 \%$ & $7 \%$ & - \\
\hline & +6 & $7 \%$ & $19 \%$ & - & - & $8 \%$ \\
\hline & +8 & $93 \%$ & $81 \%$ & - & - & $92 \%$ \\
\hline
\end{tabular}

${ }^{[a]}$ Determined from a control experiment without labelled compounds. 


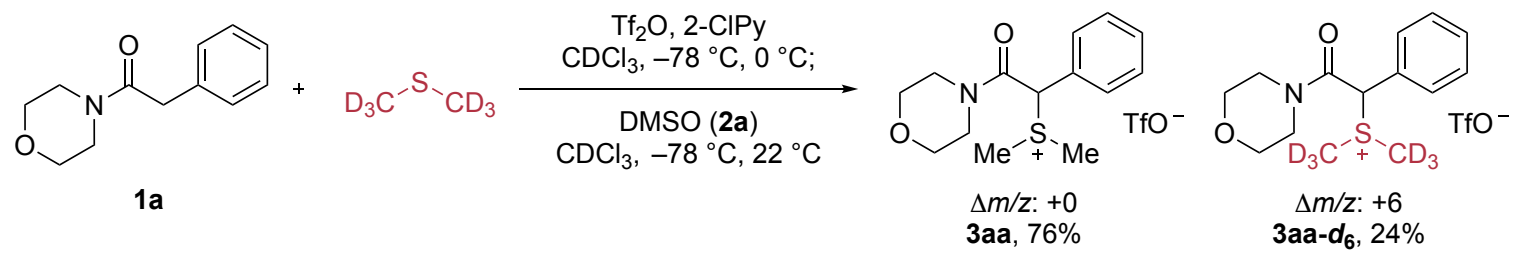

Mechanistic Experiment F. $\alpha$-Sulfidation of Morpholine Amide 1a with Dimethyl Sulfoxide (2a) in the Presence of Dimethyl Sulfide- $d_{6}$ :

Trifluoromethanesulfonic anhydride $(88.3 \mu \mathrm{L}, 525 \mu \mathrm{mol}, 1.05$ equiv) was added via syringe over $1 \mathrm{~min}$ to a stirred solution of azeotropically dried morpholine amide $1 \mathrm{a}$ (103 $\mathrm{mg}$, $500 \mu \mathrm{mol}, 1$ equiv) and 2-chloropyridine $(142 \mu \mathrm{L}, 1.50 \mathrm{mmol}, 3.00$ equiv) in dichloromethane $(1.7 \mathrm{~mL})$ at $-78^{\circ} \mathrm{C}$ under argon atmosphere. The dark red solution was stirred at $0{ }^{\circ} \mathrm{C}$ for $15 \mathrm{~min}$, before it was again cooled to $-78^{\circ} \mathrm{C}$. A solution of dimethyl sulfoxide $(\mathbf{2 a}, 42.6 \mu \mathrm{L}, 600 \mu \mathrm{mol}$, 1.20 equiv) in dichloromethane $(0.3 \mathrm{~mL})$ was added via cannula over $2 \mathrm{~min}$ at $-78^{\circ} \mathrm{C}$, followed immediately by dimethyl sulfide- $d_{6}\left(36.7 \mu \mathrm{L}, 500 \mu \mathrm{mol}, 1.00\right.$ equiv) at $-78^{\circ} \mathrm{C}$. The reaction mixture was stirred at $0{ }^{\circ} \mathrm{C}$ for $15 \mathrm{~min}$, then at $22{ }^{\circ} \mathrm{C}$ for $30 \mathrm{~min}$ to give an orange solution, which was concentrated under reduced pressure. The ratio of 3aa to 3aa- $\boldsymbol{d}_{\mathbf{6}}$ was determined to be $76 \%$ to $24 \%$ via Q-TOF mass spectrometry. 


\section{X-Ray Diffraction Data of $N, N$-Dimethyl Amide Dimethyl Sulfonium} Trifluoromethanesulfonate 3za:

Structural parameters for $N, N$-dimethyl amide dimethyl sulfonium trifluoromethanesulfonate 3za are freely available from the Cambridge Crystallographic Data Center (CCDC 1916405).
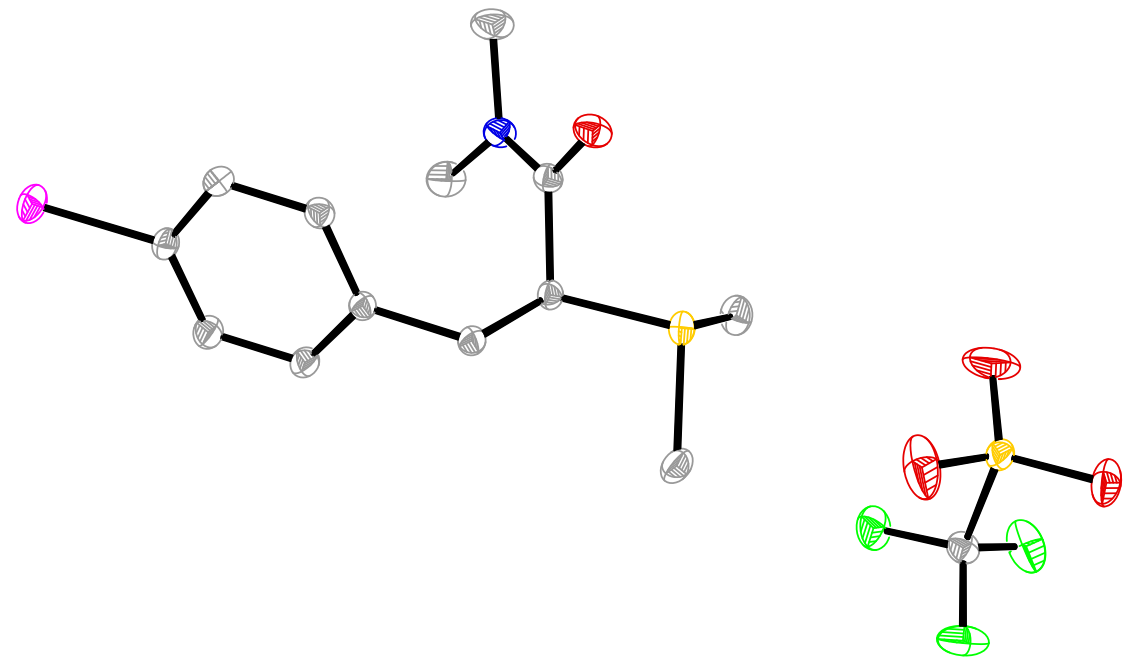

Figure S6: ORTEP representation of $N, N$-dimethyl amide dimethyl sulfonium trifluoromethanesulfonate $\mathbf{3 z a}$. Thermal ellipsoids are shown at the $50 \%$ probability level. Hydrogen atoms are omitted for clarity.

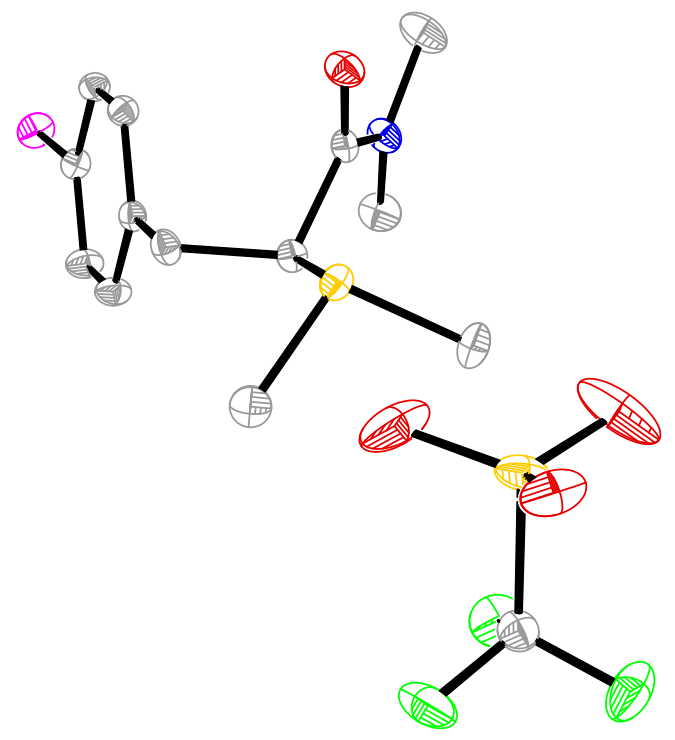

Figure S7: ORTEP representation of $N, N$-dimethyl amide dimethyl sulfonium trifluoromethanesulfonate 3za. Thermal ellipsoids are shown at the $50 \%$ probability level. Hydrogen atoms are omitted for clarity. 


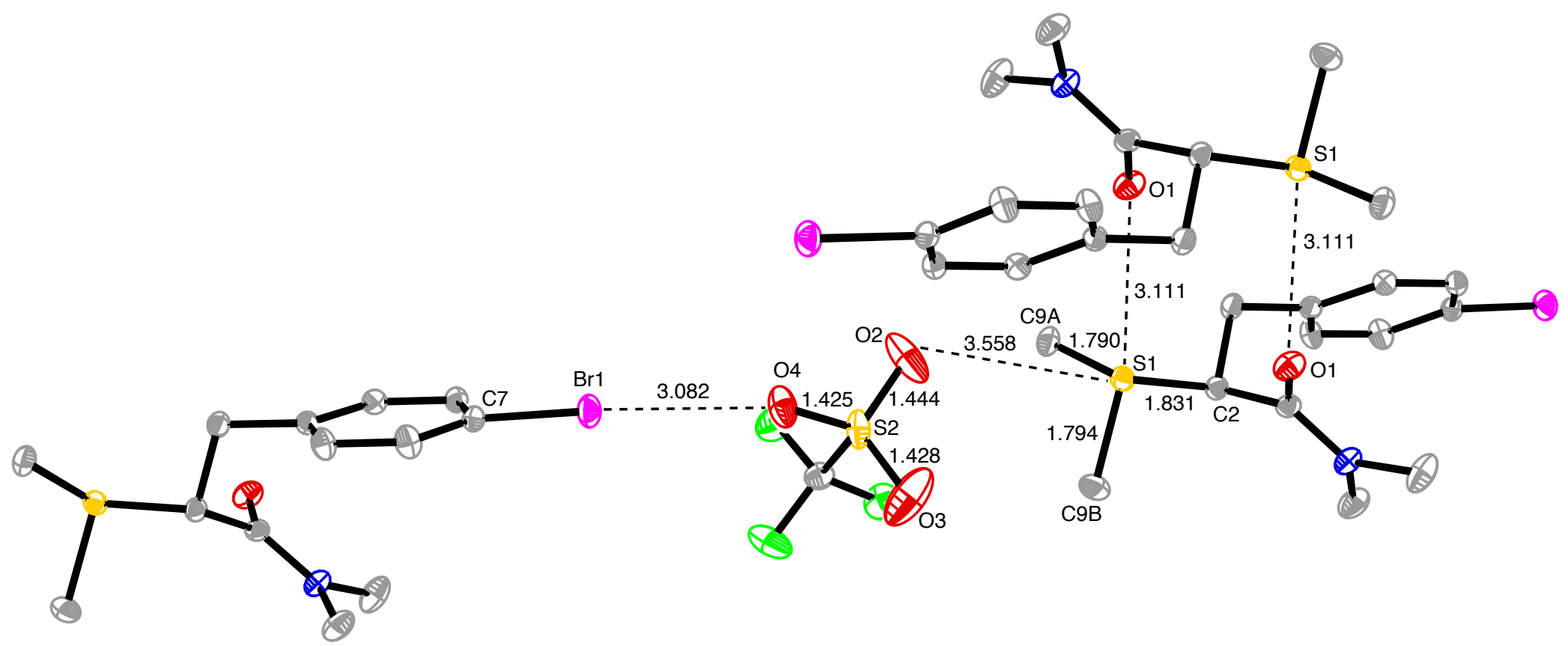

Figure S8: ORTEP representation of $N, N$-dimethyl amide dimethyl sulfonium trifluoromethanesulfonate 3za showing intermolecular, non-covalent interactions. Thermal ellipsoids are shown at the 50\% probability level. Hydrogen atoms are omitted for clarity. Selected bond lengths $[\AA]$ and angles $\left[{ }^{\circ}\right]$ : $\mathrm{S}(1)-\mathrm{C}(2) 1.831, \mathrm{~S}(1)-$ C(9A) 1.790, S(1)-C(9B) 1.794, S(1)-O(1) 3.111, S(1)-O(2) 3.558, S(2)-O(2) 1.444, S(2)-O(3) 1.428, S(2)-O(4) 1.425, O(4)-Br(1) 3.082, C(2)-S(1)-O(2) 163.79, $\mathrm{O}(1)-\mathrm{S}(1)-\mathrm{C}(9 \mathrm{~B}) 161.55, \mathrm{O}(4)-\mathrm{Br}(1)-\mathrm{C}(7) 176.69$ 
Table S7: Crystal data and structure refinement for $N, N$-dimethyl amide dimethyl sulfonium trifluoromethanesulfonate 3za.

\begin{tabular}{|c|c|c|}
\hline Identification code & CCDC 1916405 & \\
\hline Empirical formula & C14 H19 Br F3 N O4 S2 & \\
\hline Formula weight & 466.33 & \\
\hline Temperature & $100(2) \mathrm{K}$ & \\
\hline Wavelength & $0.71073 \AA$ & \\
\hline Crystal system & Monoclinic & \\
\hline Space group & $\mathrm{P} 2{ }_{1} / \mathrm{c}$ & \\
\hline \multirow[t]{3}{*}{ Unit cell dimensions } & $\mathrm{a}=11.4614(7) \AA$ & $\alpha=90^{\circ}$ \\
\hline & $b=9.9145(6) \AA$ & $\beta=108.864(2)^{\circ}$. \\
\hline & $c=17.6260(10) \AA$ & $\gamma=90^{\circ}$ \\
\hline Volume & $1895.3(2) \AA^{3}$ & \\
\hline $\mathrm{Z}$ & 4 & \\
\hline Density (calculated) & $1.634 \mathrm{Mg} / \mathrm{m}^{3}$ & \\
\hline Absorption coefficient & $2.436 \mathrm{~mm}^{-1}$ & \\
\hline $\mathrm{F}(000)$ & 944 & \\
\hline Crystal size & $0.420 \times 0.385 \times 0.260 \mathrm{~mm}^{3}$ & \\
\hline Theta range for data collection & 1.878 to $32.617^{\circ}$ & \\
\hline Index ranges & $-17<=\mathrm{h}<=17,-15<=\mathrm{k}<=15,-26<=1<=24$ & \\
\hline Reflections collected & 88966 & \\
\hline Independent reflections & $6925[\mathrm{R}($ int $)=0.0462]$ & \\
\hline Completeness to theta $=25.242^{\circ}$ & $100.0 \%$ & \\
\hline Absorption correction & Semi-empirical from equivalents & \\
\hline Max. and min. transmission & 0.7464 and 0.5675 & \\
\hline Refinement method & Full-matrix least-squares on $\mathrm{F}^{2}$ & \\
\hline Data / restraints / parameters & $6925 / 0 / 230$ & \\
\hline Goodness-of-fit on $\mathrm{F}^{2}$ & 1.030 & \\
\hline Final $R$ indices $[I>2 \operatorname{sigma}(I)]$ & $\mathrm{R} 1=0.0320, \mathrm{wR} 2=0.0778$ & \\
\hline $\mathrm{R}$ indices (all data) & $\mathrm{R} 1=0.0429, \mathrm{wR} 2=0.0824$ & \\
\hline Extinction coefficient & $\mathrm{n} / \mathrm{a}$ & \\
\hline Largest diff. peak and hole & 1.911 and -1.219 e. $\AA^{-3}$ & \\
\hline
\end{tabular}


Table S8: Atomic coordinates (x $\left.10^{4}\right)$ and equivalent isotropic displacement parameters $\left(\AA^{2} \times 10^{3}\right)$ for $N, N$-dimethyl amide dimethyl sulfonium trifluoromethanesulfonate 3za. $U(e q)$ is defined as one third of the trace of the orthogonalized $U^{\mathrm{ij}}$ tensor.

\begin{tabular}{ccccc}
\hline & $\mathbf{x}$ & $\mathbf{y}$ & $\mathbf{z}$ & $\mathbf{U}(\mathbf{e q})$ \\
\hline $\mathrm{Br}(1)$ & $292(1)$ & $534(1)$ & $6505(1)$ & $22(1)$ \\
$\mathrm{S}(1)$ & $4823(1)$ & $3901(1)$ & $3840(1)$ & $16(1)$ \\
$\mathrm{O}(1)$ & $3297(1)$ & $5447(1)$ & $4483(1)$ & $21(1)$ \\
$\mathrm{N}(1)$ & $1558(1)$ & $4211(1)$ & $3968(1)$ & $18(1)$ \\
$\mathrm{C}(1)$ & $2781(1)$ & $4375(2)$ & $4211(1)$ & $15(1)$ \\
$\mathrm{C}(2)$ & $3608(1)$ & $3168(2)$ & $4177(1)$ & $14(1)$ \\
$\mathrm{C}(3)$ & $4184(1)$ & $2550(2)$ & $5018(1)$ & $16(1)$ \\
$\mathrm{C}(4)$ & $3218(1)$ & $2058(2)$ & $5366(1)$ & $14(1)$ \\
$\mathrm{C}(5 \mathrm{~B})$ & $2739(1)$ & $2926(2)$ & $5815(1)$ & $16(1)$ \\
$\mathrm{C}(5 \mathrm{~A})$ & $2781(2)$ & $736(2)$ & $5241(1)$ & $19(1)$ \\
$\mathrm{C}(6 \mathrm{~B})$ & $1851(1)$ & $2477(2)$ & $6141(1)$ & $16(1)$ \\
$\mathrm{C}(6 \mathrm{~A})$ & $1898(2)$ & $270(2)$ & $5568(1)$ & $20(1)$ \\
$\mathrm{C}(7)$ & $1450(1)$ & $1152(2)$ & $6018(1)$ & $16(1)$ \\
$\mathrm{C}(8 \mathrm{E})$ & $881(2)$ & $2961(2)$ & $3697(1)$ & $25(1)$ \\
$\mathrm{C}(8 \mathrm{Z})$ & $811(2)$ & $5348(2)$ & $4070(1)$ & $29(1)$ \\
$\mathrm{C}(9 \mathrm{~B})$ & $4057(2)$ & $3887(2)$ & $2777(1)$ & $24(1)$ \\
$\mathrm{C}(9 \mathrm{~A})$ & $5901(2)$ & $2545(2)$ & $3969(1)$ & $21(1)$ \\
$\mathrm{S}(2)$ & $7255(1)$ & $4728(1)$ & $2349(1)$ & $22(1)$ \\
$\mathrm{F}(1)$ & $7027(1)$ & $3275(1)$ & $1077(1)$ & $36(1)$ \\
$\mathrm{F}(2)$ & $8107(1)$ & $2334(1)$ & $2172(1)$ & $34(1)$ \\
$\mathrm{F}(3)$ & $6140(1)$ & $2416(1)$ & $1883(1)$ & $31(1)$ \\
$\mathrm{O}(2)$ & $7407(2)$ & $4351(2)$ & $3166(1)$ & $46(1)$ \\
$\mathrm{O}(3)$ & $6112(2)$ & $5372(2)$ & $1936(2)$ & $57(1)$ \\
$\mathrm{O}(4)$ & $8306(1)$ & $5352(1)$ & $2234(1)$ & $29(1)$ \\
$\mathrm{C}(10)$ & $7130(2)$ & $3101(2)$ & $1849(1)$ & $20(1)$ \\
\hline & & & &
\end{tabular}


Table S9: Bond lengths $[\AA]$ and angles $\left[^{\circ}\right]$ for $N, N$-dimethyl amide dimethyl sulfonium trifluoromethanesulfonate $\mathbf{3 z a}$.

\begin{tabular}{|c|c|c|c|}
\hline $\operatorname{Br}(1)-C(7)$ & $1.8994(15)$ & $\mathrm{C}(9 \mathrm{~A})-\mathrm{S}(1)-\mathrm{C}(9 \mathrm{~B})$ & $102.19(9)$ \\
\hline$S(1)-C(9 A)$ & $1.7901(18)$ & $\mathrm{C}(9 \mathrm{~A})-\mathrm{S}(1)-\mathrm{C}(2)$ & $103.12(8)$ \\
\hline $\mathrm{S}(1)-\mathrm{C}(9 \mathrm{~B})$ & $1.7939(17)$ & $\mathrm{C}(9 \mathrm{~B})-\mathrm{S}(1)-\mathrm{C}(2)$ & $99.71(8)$ \\
\hline $\mathrm{S}(1)-\mathrm{C}(2)$ & $1.8313(15)$ & $\mathrm{C}(1)-\mathrm{N}(1)-\mathrm{C}(8 \mathrm{E})$ & $126.86(14)$ \\
\hline $\mathrm{O}(1)-\mathrm{C}(1)$ & $1.2350(19)$ & $\mathrm{C}(1)-\mathrm{N}(1)-\mathrm{C}(8 \mathrm{Z})$ & $117.57(14)$ \\
\hline $\mathrm{N}(1)-\mathrm{C}(1)$ & $1.336(2)$ & $\mathrm{C}(8 \mathrm{E})-\mathrm{N}(1)-\mathrm{C}(8 \mathrm{Z})$ & $115.14(14)$ \\
\hline $\mathrm{N}(1)-\mathrm{C}(8 \mathrm{E})$ & $1.459(2)$ & $\mathrm{O}(1)-\mathrm{C}(1)-\mathrm{N}(1)$ & $123.35(15)$ \\
\hline $\mathrm{N}(1)-\mathrm{C}(8 \mathrm{Z})$ & $1.461(2)$ & $\mathrm{O}(1)-\mathrm{C}(1)-\mathrm{C}(2)$ & $117.44(14)$ \\
\hline $\mathrm{C}(1)-\mathrm{C}(2)$ & $1.540(2)$ & $\mathrm{N}(1)-\mathrm{C}(1)-\mathrm{C}(2)$ & $119.19(14)$ \\
\hline $\mathrm{C}(2)-\mathrm{C}(3)$ & $1.542(2)$ & $\mathrm{C}(1)-\mathrm{C}(2)-\mathrm{C}(3)$ & $110.41(12)$ \\
\hline$C(3)-C(4)$ & $1.510(2)$ & $\mathrm{C}(1)-\mathrm{C}(2)-\mathrm{S}(1)$ & $104.24(10)$ \\
\hline$C(4)-C(5 A)$ & $1.396(2)$ & $\mathrm{C}(3)-\mathrm{C}(2)-\mathrm{S}(1)$ & $109.54(10)$ \\
\hline$C(4)-C(5 B)$ & $1.396(2)$ & $C(4)-C(3)-C(2)$ & $112.09(12)$ \\
\hline $\mathrm{C}(5 \mathrm{~B})-\mathrm{C}(6 \mathrm{~B})$ & $1.393(2)$ & $C(5 A)-C(4)-C(5 B)$ & $118.78(14)$ \\
\hline$C(5 A)-C(6 A)$ & $1.395(2)$ & $\mathrm{C}(5 \mathrm{~A})-\mathrm{C}(4)-\mathrm{C}(3)$ & $120.81(14)$ \\
\hline $\mathrm{C}(6 \mathrm{~B})-\mathrm{C}(7)$ & $1.386(2)$ & $C(5 B)-C(4)-C(3)$ & $120.41(14)$ \\
\hline$C(6 A)-C(7)$ & $1.386(2)$ & $C(6 B)-C(5 B)-C(4)$ & $120.74(14)$ \\
\hline $\mathrm{S}(2)-\mathrm{O}(4)$ & $1.4249(14)$ & $C(6 \mathrm{~A})-\mathrm{C}(5 \mathrm{~A})-\mathrm{C}(4)$ & $121.12(15)$ \\
\hline $\mathrm{S}(2)-\mathrm{O}(3)$ & $1.4276(18)$ & $C(7)-C(6 B)-C(5 B)$ & $119.18(14)$ \\
\hline $\mathrm{S}(2)-\mathrm{O}(2)$ & $1.4437(17)$ & $C(7)-C(6 A)-C(5 A)$ & $118.74(15)$ \\
\hline $\mathrm{S}(2)-\mathrm{C}(10)$ & $1.8212(17)$ & $C(6 A)-C(7)-C(6 B)$ & $121.43(14)$ \\
\hline $\mathrm{F}(1)-\mathrm{C}(10)$ & $1.338(2)$ & $\mathrm{C}(6 \mathrm{~A})-\mathrm{C}(7)-\mathrm{Br}(1)$ & $119.85(12)$ \\
\hline $\mathrm{F}(2)-\mathrm{C}(10)$ & $1.321(2)$ & $C(6 B)-C(7)-B r(1)$ & $118.70(12)$ \\
\hline \multirow[t]{12}{*}{$\mathrm{F}(3)-\mathrm{C}(10)$} & $1.340(2)$ & $\mathrm{O}(4)-\mathrm{S}(2)-\mathrm{O}(3)$ & $114.34(11)$ \\
\hline & & $\mathrm{O}(4)-\mathrm{S}(2)-\mathrm{O}(2)$ & $114.80(11)$ \\
\hline & & $\mathrm{O}(3)-\mathrm{S}(2)-\mathrm{O}(2)$ & $114.75(14)$ \\
\hline & & $\mathrm{O}(4)-\mathrm{S}(2)-\mathrm{C}(10)$ & $104.71(8)$ \\
\hline & & $\mathrm{O}(3)-\mathrm{S}(2)-\mathrm{C}(10)$ & $103.51(11)$ \\
\hline & & $\mathrm{O}(2)-\mathrm{S}(2)-\mathrm{C}(10)$ & $102.65(9)$ \\
\hline & & $\mathrm{F}(2)-\mathrm{C}(10)-\mathrm{F}(1)$ & $107.21(15)$ \\
\hline & & $\mathrm{F}(2)-\mathrm{C}(10)-\mathrm{F}(3)$ & $107.53(14)$ \\
\hline & & $\mathrm{F}(1)-\mathrm{C}(10)-\mathrm{F}(3)$ & $108.26(14)$ \\
\hline & & $F(2)-C(10)-S(2)$ & $112.23(12)$ \\
\hline & & $\mathrm{F}(1)-\mathrm{C}(10)-\mathrm{S}(2)$ & $110.18(12)$ \\
\hline & & $\mathrm{F}(3)-\mathrm{C}(10)-\mathrm{S}(2)$ & $111.25(12)$ \\
\hline
\end{tabular}

Symmetry transformations used to generate equivalent atoms. 
Table S10: Anisotropic displacement parameters $\left(\AA^{2} \times 10^{3}\right)$ for $N, N$-dimethyl amide dimethyl sulfonium trifluoromethanesulfonate 3za. The anisotropic displacement factor exponent takes the form: $-2 \pi^{2}\left[\mathrm{~h}^{2} \mathrm{a}^{* 2} \mathrm{U}^{11}+\ldots+2 \mathrm{hk} \mathrm{a} \mathrm{b}^{*} \mathrm{U}^{12}\right]$.

\begin{tabular}{ccccccc}
\hline & $\mathbf{U}^{\mathbf{1 1}}$ & $\mathbf{U}^{\mathbf{2 2}}$ & $\mathbf{U}^{\mathbf{3 3}}$ & $\mathbf{U}^{\mathbf{2 3}}$ & $\mathbf{U}^{\mathbf{1 3}}$ & $\mathbf{U}^{\mathbf{1 2}}$ \\
\hline $\mathrm{Br}(1)$ & $20(1)$ & $26(1)$ & $25(1)$ & $-1(1)$ & $13(1)$ & $-6(1)$ \\
$\mathrm{S}(1)$ & $16(1)$ & $17(1)$ & $15(1)$ & $0(1)$ & $6(1)$ & $-4(1)$ \\
$\mathrm{O}(1)$ & $18(1)$ & $16(1)$ & $26(1)$ & $-2(1)$ & $3(1)$ & $-2(1)$ \\
$\mathrm{N}(1)$ & $13(1)$ & $17(1)$ & $22(1)$ & $1(1)$ & $4(1)$ & $0(1)$ \\
$\mathrm{C}(1)$ & $14(1)$ & $16(1)$ & $14(1)$ & $2(1)$ & $3(1)$ & $2(1)$ \\
$\mathrm{C}(2)$ & $13(1)$ & $15(1)$ & $15(1)$ & $1(1)$ & $5(1)$ & $-1(1)$ \\
$\mathrm{C}(3)$ & $14(1)$ & $18(1)$ & $18(1)$ & $5(1)$ & $7(1)$ & $3(1)$ \\
$\mathrm{C}(4)$ & $14(1)$ & $16(1)$ & $14(1)$ & $2(1)$ & $5(1)$ & $1(1)$ \\
$\mathrm{C}(5 \mathrm{~B})$ & $16(1)$ & $14(1)$ & $17(1)$ & $0(1)$ & $5(1)$ & $-1(1)$ \\
$\mathrm{C}(5 \mathrm{~A})$ & $23(1)$ & $16(1)$ & $21(1)$ & $-1(1)$ & $13(1)$ & $0(1)$ \\
$\mathrm{C}(6 \mathrm{~B})$ & $15(1)$ & $17(1)$ & $16(1)$ & $-1(1)$ & $7(1)$ & $1(1)$ \\
$\mathrm{C}(6 \mathrm{~A})$ & $25(1)$ & $15(1)$ & $24(1)$ & $-3(1)$ & $13(1)$ & $-4(1)$ \\
$\mathrm{C}(7)$ & $14(1)$ & $18(1)$ & $16(1)$ & $1(1)$ & $6(1)$ & $-2(1)$ \\
$\mathrm{C}(8 \mathrm{E})$ & $16(1)$ & $25(1)$ & $32(1)$ & $-5(1)$ & $3(1)$ & $-4(1)$ \\
$\mathrm{C}(8 \mathrm{Z})$ & $17(1)$ & $23(1)$ & $46(1)$ & $1(1)$ & $10(1)$ & $5(1)$ \\
$\mathrm{C}(9 \mathrm{~B})$ & $25(1)$ & $31(1)$ & $14(1)$ & $3(1)$ & $6(1)$ & $-1(1)$ \\
$\mathrm{C}(9 \mathrm{~A})$ & $15(1)$ & $27(1)$ & $24(1)$ & $1(1)$ & $10(1)$ & $0(1)$ \\
$\mathrm{S}(2)$ & $25(1)$ & $14(1)$ & $34(1)$ & $-3(1)$ & $20(1)$ & $-2(1)$ \\
$\mathrm{F}(1)$ & $56(1)$ & $32(1)$ & $19(1)$ & $-4(1)$ & $10(1)$ & $-14(1)$ \\
$\mathrm{F}(2)$ & $30(1)$ & $24(1)$ & $44(1)$ & $-4(1)$ & $5(1)$ & $10(1)$ \\
$\mathrm{F}(3)$ & $29(1)$ & $26(1)$ & $38(1)$ & $-4(1)$ & $11(1)$ & $-13(1)$ \\
$\mathrm{O}(2)$ & $86(1)$ & $31(1)$ & $36(1)$ & $-13(1)$ & $41(1)$ & $-22(1)$ \\
$\mathrm{O}(3)$ & $30(1)$ & $27(1)$ & $110(2)$ & $5(1)$ & $16(1)$ & $12(1)$ \\
$\mathrm{O}(4)$ & $35(1)$ & $25(1)$ & $36(1)$ & $-10(1)$ & $24(1)$ & $-14(1)$ \\
$\mathrm{C}(10)$ & $20(1)$ & $17(1)$ & $21(1)$ & $-1(1)$ & $4(1)$ & $-2(1)$ \\
\hline & & & & & & \\
& & & & & \\
& & & & & \\
& & & &
\end{tabular}


Table S11: Hydrogen coordinates (x 10 $0^{4}$ and isotropic displacement parameters $\left(\AA^{2} \times 10^{3}\right)$ for $N, N$-dimethyl amide dimethyl sulfonium trifluoromethanesulfonate 3za.

\begin{tabular}{ccccc}
\hline & $\mathbf{x}$ & $\mathbf{y}$ & $\mathbf{z}$ & $\mathbf{U}(\mathbf{e q})$ \\
\hline $\mathrm{H}(2)$ & 3131 & 2473 & 3788 & 17 \\
$\mathrm{H}(3 \mathrm{~A})$ & 4722 & 1786 & 4985 & 20 \\
$\mathrm{H}(3 \mathrm{~B})$ & 4705 & 3236 & 5380 & 20 \\
$\mathrm{H}(5 \mathrm{~B})$ & 3020 & 3832 & 5898 & 19 \\
$\mathrm{H}(5 \mathrm{~A})$ & 3090 & 142 & 4928 & 23 \\
$\mathrm{H}(6 \mathrm{~B})$ & 1526 & 3072 & 6445 & 19 \\
$\mathrm{H}(6 \mathrm{~A})$ & 1610 & -634 & 5484 & 24 \\
$\mathrm{H}(8 \mathrm{E} 1)$ & 1413 & 2316 & 3543 & 38 \\
$\mathrm{H}(8 \mathrm{E} 2)$ & 151 & 3148 & 3233 & 38 \\
$\mathrm{H}(8 \mathrm{E} 3)$ & 626 & 2579 & 4131 & 38 \\
$\mathrm{H}(8 \mathrm{Z} 1)$ & 1335 & 6147 & 4237 & 44 \\
$\mathrm{H}(8 \mathrm{Z} 2)$ & 434 & 5126 & 4480 & 44 \\
$\mathrm{H}(8 \mathrm{Z} 3)$ & 163 & 5533 & 3561 & 44 \\
$\mathrm{H}(9 \mathrm{~B} 1)$ & 4611 & 4251 & 2505 & 36 \\
$\mathrm{H}(9 \mathrm{~B} 2)$ & 3312 & 4443 & 2648 & 36 \\
$\mathrm{H}(9 \mathrm{~B} 3)$ & 3831 & 2959 & 2598 & 36 \\
$\mathrm{H}(9 \mathrm{~A} 1)$ & 5455 & 1706 & 3770 & 32 \\
$\mathrm{H}(9 \mathrm{~A} 2)$ & 6372 & 2447 & 4539 & 32 \\
$\mathrm{H}(9 \mathrm{~A} 3)$ & 6466 & 2740 & 3668 & 32 \\
\hline
\end{tabular}


Computational Information. All computational studies were conducted using the Gaussian09 software package. ${ }^{21}$ Geometry optimizations of all structures (local minima (LMs) and transition states (TSs)) were performed with M06-2X as hybrid meta exchange-correlation functional ${ }^{22}$ together with the $6-31+\mathrm{G}(\mathrm{d}, \mathrm{p})$ split-valence basis set of Pople et al. for all atoms. ${ }^{23}$ Solvent effects were considered by using the self-consistent reaction field (SCRF) method based on the conductorlike polarizable continuum model $(\mathrm{CPCM})$ for dichloromethane $\left(\mathrm{CH}_{2} \mathrm{Cl}_{2}\right)$ with a dielectric constant $\varepsilon=8.93 .{ }^{24}$ The connectivity of TS structures was confirmed by intrinsic reaction coordinate (IRC) analyses at the same computational level. All stationary points (LMs and TSs) were characterized by harmonic frequency calculations, yielding none or one imaginary frequency, respectively. Single-point energy calculations were carried out on the $\mathrm{CPCM}\left(\mathrm{CH}_{2} \mathrm{Cl}_{2}\right) \mathrm{M} 06-2 \mathrm{X} / 6-$ $31+\mathrm{G}(\mathrm{d}, \mathrm{p})$ optimized geometries at the $\mathrm{CPCM}\left(\mathrm{CH}_{2} \mathrm{Cl}_{2}\right) \mathrm{M} 06-2 \mathrm{X} / 6-311+\mathrm{G}(2 \mathrm{df}, \mathrm{p})$ level of theory. The relative Gibbs free energies $\left(\Delta \Delta G^{0}\right)$ at $298.15 \mathrm{~K}$ and 1 atm are reported in kcal.mol ${ }^{-1}$. All computed structures were visualized using the Gabedit software package. ${ }^{25}$ 


\section{Computed Reaction Profiles for the $\alpha$-Sulfidation of Benzylic Amides:}

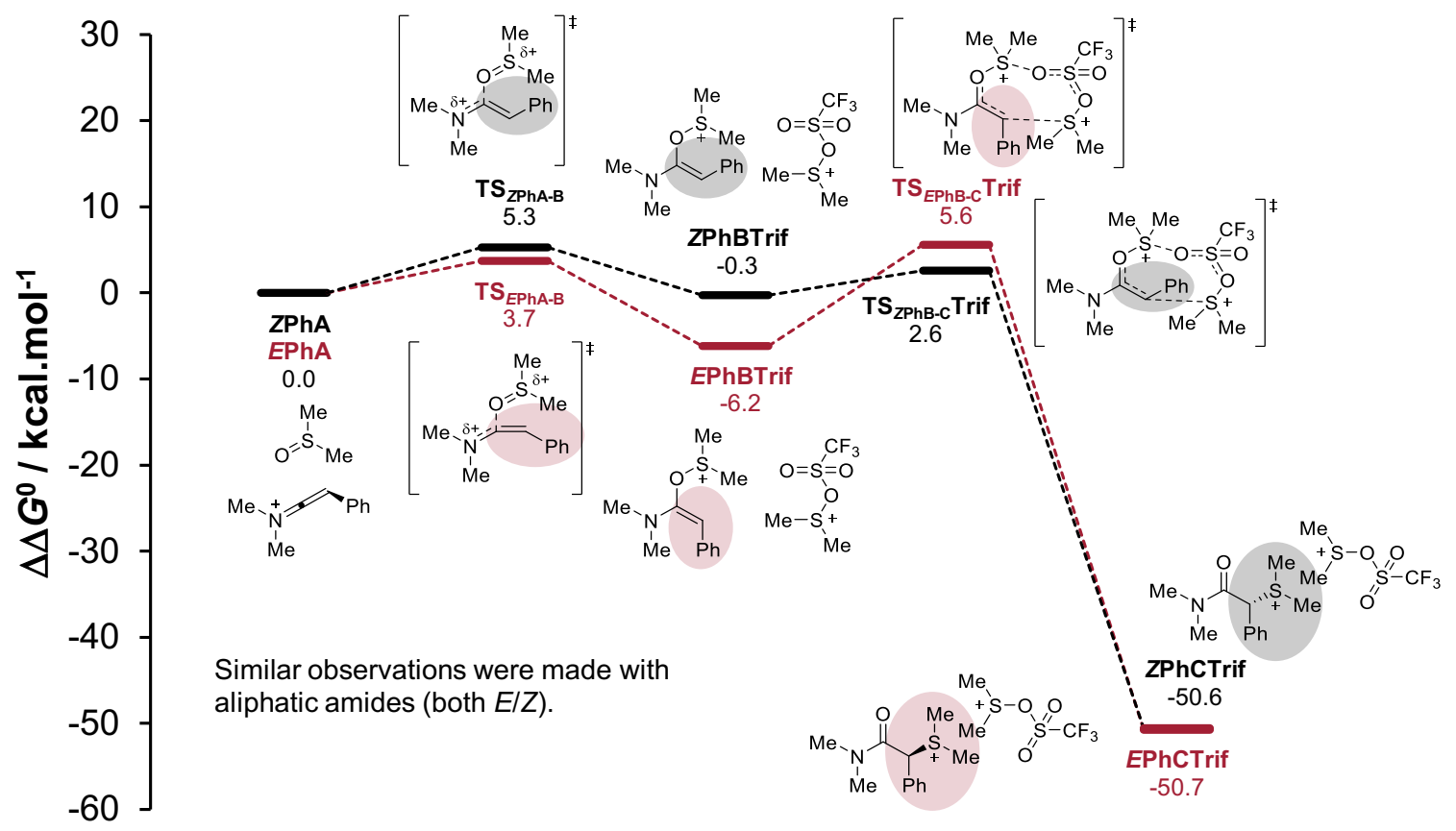

Figure S9: Computed reaction profile (path B) for the $\alpha$-sulfidation of benzylic amides at the $\mathrm{CPCM}\left(\mathrm{CH}_{2} \mathrm{Cl}_{2}\right) \mathrm{M} 06-2 \mathrm{X} / 6$ $311+\mathrm{G}(2 \mathrm{df}, \mathrm{p}) / / \mathrm{CPCM}\left(\mathrm{CH}_{2} \mathrm{Cl}_{2}\right) \mathrm{M} 06-2 \mathrm{X} / 6-31+\mathrm{G}(\mathrm{d}, \mathrm{p})$ level of theory. Relative free energies $\Delta \Delta \mathrm{G}^{0}$ are in relation to $\boldsymbol{Z P h A}$ $((Z)$-stereoisomer) and $\boldsymbol{E} \mathbf{P h A}((E)$-stereoisomer $)$ as the starting points.

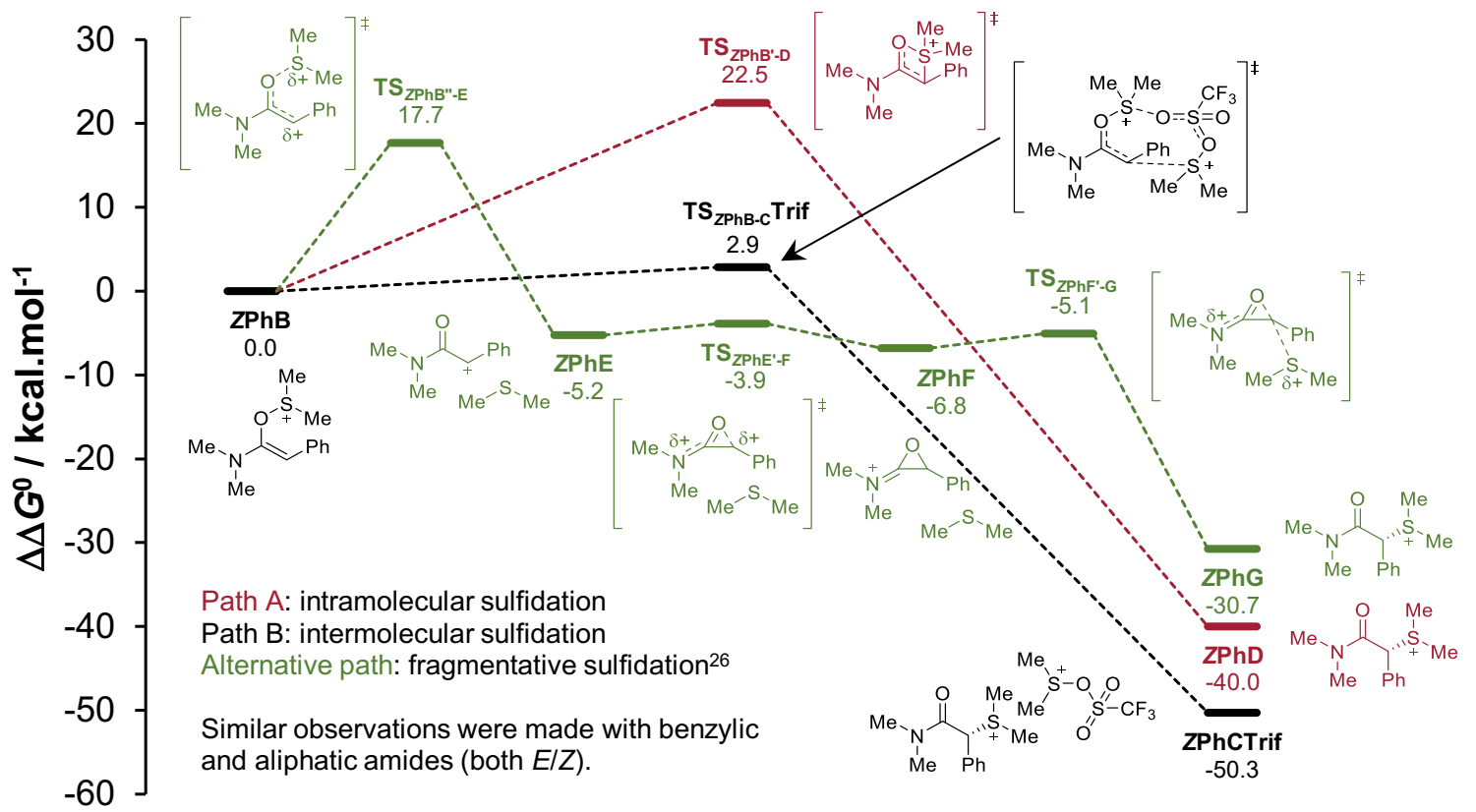

Figure S10: Computed reaction profiles for the $\alpha$-sulfidation of benzylic amides at the $\mathrm{CPCM}\left(\mathrm{CH}_{2} \mathrm{Cl}_{2}\right) \mathrm{M} 06-2 \mathrm{X} / 6$ $311+\mathrm{G}(2 \mathrm{df}, \mathrm{p}) / / \mathrm{CPCM}\left(\mathrm{CH}_{2} \mathrm{Cl}_{2}\right) \mathrm{M} 06-2 \mathrm{X} / 6-31+\mathrm{G}(\mathrm{d}, \mathrm{p})$ level of theory. Relative free energies $\Delta \Delta \mathrm{G}^{0}$ are in relation to $\mathbf{Z P h B}$ as the starting point. ${ }^{27}$ 


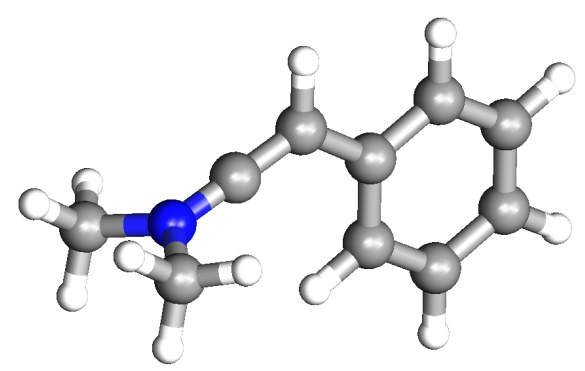

$\mathrm{E}=-442.435795$ a.u. (0 imaginary frequencies)

$\begin{array}{lrrrrrrrr}\mathrm{C} & -0.152506 & 0.020444 & -0.210832 & \mathrm{C} & -1.363081 & 0.017646 & 1.944219 \\ \mathrm{C} & -0.256057 & 0.032116 & 1.259105 & \mathrm{~N} & -2.420609 & -0.001243 & 2.612890 \\ \mathrm{C} & 1.120893 & -0.030893 & -0.786716 & \mathrm{C} & -3.114904 & 1.243933 & 3.020241 \\ \mathrm{C} & 1.256107 & -0.047865 & -2.173225 & \mathrm{C} & -3.054701 & -1.271578 & 3.040158 \\ \mathrm{C} & 0.124236 & -0.012383 & -2.985326 & \mathrm{H} & -4.054871 & -1.306225 & 2.607256 \\ \mathrm{C} & -1.148578 & 0.040842 & -2.410703 & \mathrm{H} & -2.450137 & -2.104439 & 2.689842 \\ \mathrm{C} & -1.289861 & 0.057682 & -1.028938 & \mathrm{H} & -3.114373 & -1.263473 & 4.128779 \\ \mathrm{H} & 2.001527 & -0.058881 & -0.152097 & \mathrm{H} & -4.116906 & 1.221561 & 2.590445 \\ \mathrm{H} & 2.245607 & -0.089375 & -2.616095 & \mathrm{H} & -3.171891 & 1.252289 & 4.108999 \\ \mathrm{H} & 0.230658 & -0.025047 & -4.065087 & \mathrm{H} & -2.552941 & 2.099305 & 2.653377 \\ \mathrm{H} & -2.030419 & 0.069834 & -3.041822 & \mathrm{H} & 0.657089 & 0.051849 & 1.854138 \\ \mathrm{H} & -2.282167 & 0.103901 & -0.587373 & & & & \end{array}$




\section{Optimized Cartesian Coordinates and Zero Point Vibrational Energy Corrected Absolute} Energy in Hartree of DMSO (Calculated with $\mathrm{CPCM}\left(\mathrm{CH}_{2} \mathrm{Cl}_{2}\right) \mathrm{M06-2X/6-31+G(d,p)):}$

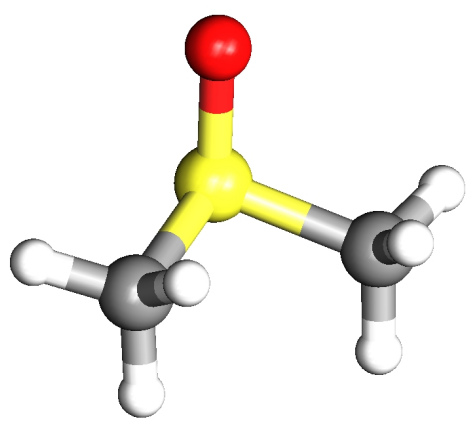

$\mathrm{E}=-553.015342$ a.u. (0 imaginary frequencies)

$\begin{array}{lrrrrrrrr}\mathrm{C} & 0.038284 & -0.008592 & 0.021272 & \mathrm{H} & 2.101791 & -0.900548 & 1.851456 \\ \mathrm{~S} & -0.151292 & -0.072300 & 1.816962 & \mathrm{H} & 2.034529 & 0.899638 & 1.806725 \\ \mathrm{C} & 1.604722 & -0.007995 & 2.236571 & \mathrm{H} & -0.963890 & 0.019848 & -0.407730 \\ \mathrm{O} & -0.725972 & 1.282653 & 2.223435 & \mathrm{H} & 0.589860 & 0.897022 & -0.240786 \\ \mathrm{H} & 1.675434 & 0.017716 & 3.324371 & \mathrm{H} & 0.564973 & -0.902697 & -0.318306\end{array}$


Optimized Cartesian Coordinates and Zero Point Vibrational Energy Corrected Absolute Energy in Hartree of DMSOTrif (Calculated with CPCM(CH $\left.2 \mathrm{Cl}_{2}\right) \mathrm{M06-2X/6-31+G(d,p)):}$

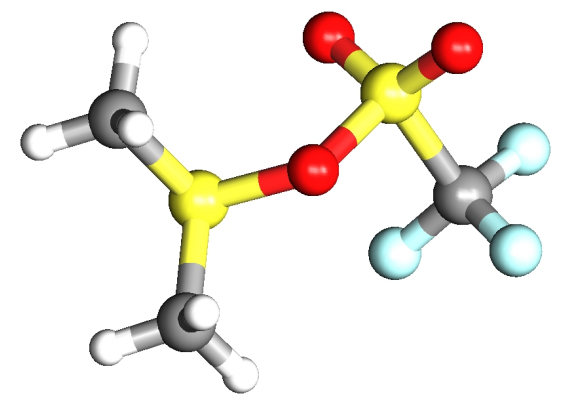

$\mathrm{E}=-1438.797418$ a.u. (0 imaginary frequencies)

$\begin{array}{lrlllllll}\mathrm{C} & 2.185998 & 0.623545 & 0.881099 & \mathrm{~F} & -3.511917 & 2.194652 & -0.593990 \\ \mathrm{~S} & 1.110277 & 1.182318 & -0.434799 & \mathrm{~F} & -2.110079 & 3.841275 & -0.736692 \\ \mathrm{C} & 2.134057 & 2.363875 & -1.307558 & \mathrm{H} & 2.929705 & 1.782977 & -1.780315 \\ \mathrm{O} & 0.188892 & 2.298576 & 0.391230 & \mathrm{H} & 1.509743 & 2.835996 & -2.065748 \\ \mathrm{~S} & -1.367219 & 1.888086 & 0.830497 & \mathrm{H} & 2.533332 & 3.084008 & -0.593470 \\ \mathrm{O} & -1.666919 & 2.736479 & 1.942620 & \mathrm{H} & 2.624778 & 1.486369 & 1.382574 \\ \mathrm{C} & -2.243776 & 2.533869 & -0.684781 & \mathrm{H} & 1.582676 & 0.014003 & 1.554187 \\ \mathrm{~F} & -1.688461 & 1.970789 & -1.746498 & \mathrm{H} & 2.948825 & 0.003009 & 0.402924 \\ \mathrm{O} & -1.454813 & 0.452716 & 0.830202 & & & & \end{array}$




\section{Optimized Cartesian Coordinates and Zero Point Vibrational Energy Corrected Absolute} Energy in Hartree of $Z \mathrm{PhA}$ (Calculated with $\mathrm{CPCM}\left(\mathrm{CH}_{2} \mathrm{Cl}_{2}\right) \mathrm{M06-2X/6-31+G(d,p)):}$

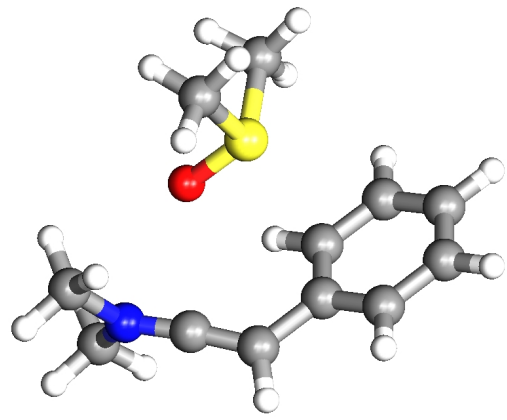

$\mathrm{E}=-995.462835$ a.u. (0 imaginary frequencies)

$\begin{array}{lrlllllll}\mathrm{C} & 2.354683 & -0.347353 & 3.293654 & \mathrm{H} & 0.544499 & 1.733837 & 3.271173 \\ \mathrm{~N} & 1.925989 & 0.576024 & 2.218487 & \mathrm{H} & 2.222586 & 2.372843 & 3.233285 \\ \mathrm{C} & 1.428135 & 1.899593 & 2.654662 & \mathrm{H} & 1.163982 & 2.480781 & 1.776747 \\ \mathrm{C} & 1.966063 & 0.242674 & 1.013102 & \mathrm{H} & 3.157376 & 0.135645 & 3.851460 \\ \mathrm{C} & 2.112681 & -0.130434 & -0.224983 & \mathrm{H} & 2.698805 & -1.277661 & 2.847731 \\ \mathrm{C} & 1.114966 & -0.866635 & -1.022918 & \mathrm{H} & 1.496753 & -0.523219 & 3.943776 \\ \mathrm{O} & -0.696328 & 0.987985 & 0.965442 & \mathrm{C} & 1.149872 & -0.747091 & -2.415617 \\ \mathrm{~S} & -1.595370 & 1.100081 & -0.269766 & \mathrm{C} & 0.203729 & -1.409741 & -3.194731 \\ \mathrm{C} & -2.279166 & 2.770655 & -0.211681 & \mathrm{C} & -0.765543 & -2.206599 & -2.587477 \\ \mathrm{C} & -3.107197 & 0.210118 & 0.155497 & \mathrm{C} & -0.784264 & -2.347387 & -1.197492 \\ \mathrm{H} & 3.061297 & 0.148911 & -0.683730 & \mathrm{C} & 0.150219 & -1.678963 & -0.413945 \\ \mathrm{H} & -2.714398 & 2.938182 & 0.775832 & \mathrm{H} & 1.908720 & -0.128365 & -2.885703 \\ \mathrm{H} & -1.454759 & 3.462473 & -0.387626 & \mathrm{H} & 0.228299 & -1.306875 & -4.274523 \\ \mathrm{H} & -3.030407 & 2.878878 & -0.996521 & \mathrm{H} & -1.497863 & -2.729148 & -3.194471 \\ \mathrm{H} & -3.841010 & 0.344045 & -0.641936 & \mathrm{H} & -1.525627 & -2.985086 & -0.725962 \\ \mathrm{H} & -3.482944 & 0.591279 & 1.107582 & \mathrm{H} & 0.134929 & -1.783989 & 0.667149 \\ \mathrm{H} & -2.841305 & -0.843850 & 0.244327 & & & & \end{array}$


Optimized Cartesian Coordinates and Zero Point Vibrational Energy Corrected Absolute Energy in Hartree of TS

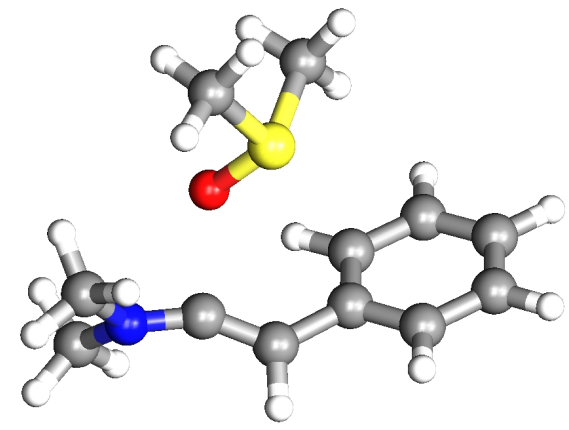

$\mathrm{E}=-995.458215$ a.u. (1 imaginary frequency)

$\begin{array}{lrrrrrrrr}\mathrm{C} & 4.819765 & -4.767250 & -0.098501 & \mathrm{H} & 3.234456 & -2.429808 & -0.428479 \\ \mathrm{~N} & 4.454598 & -3.922199 & -1.248787 & \mathrm{H} & 4.996967 & -2.120531 & -0.347052 \\ \mathrm{C} & 4.183944 & -2.506003 & -0.963552 & \mathrm{H} & 4.133810 & -1.955777 & -1.901568 \\ \mathrm{C} & 4.132880 & -4.432181 & -2.370001 & \mathrm{H} & 5.739699 & -4.374549 & 0.336981 \\ \mathrm{C} & 4.407397 & -5.008864 & -3.515040 & \mathrm{H} & 4.969032 & -5.789346 & -0.440530 \\ \mathrm{C} & 3.539808 & -5.762903 & -4.437486 & \mathrm{H} & 4.013263 & -4.727050 & 0.637371 \\ \mathrm{O} & 2.046071 & -4.236331 & -2.219011 & \mathrm{C} & 3.751837 & -5.630564 & -5.814875 \\ \mathrm{~S} & 1.248017 & -3.658752 & -3.419562 & \mathrm{C} & 2.958588 & -6.335756 & -6.716151 \\ \mathrm{C} & 0.637614 & -2.074530 & -2.820662 & \mathrm{C} & 1.968061 & -7.199011 & -6.247479 \\ \mathrm{C} & -0.303662 & -4.573014 & -3.397612 & \mathrm{C} & 1.776935 & -7.358811 & -4.874140 \\ \mathrm{H} & 5.456804 & -4.888454 & -3.790225 & \mathrm{C} & 2.556649 & -6.643197 & -3.968994 \\ \mathrm{H} & 0.159947 & -2.226220 & -1.850829 & \mathrm{H} & 4.528632 & -4.963629 & -6.177815 \\ \mathrm{H} & 1.495803 & -1.408169 & -2.728690 & \mathrm{H} & 3.118751 & -6.216461 & -7.782738 \\ \mathrm{H} & -0.067125 & -1.671991 & -3.550877 & \mathrm{H} & 1.355653 & -7.756203 & -6.949137 \\ \mathrm{H} & -0.977397 & -4.135395 & -4.136781 & \mathrm{H} & 1.021514 & -8.046190 & -4.505855 \\ \mathrm{H} & -0.726241 & -4.517634 & -2.392700 & \mathrm{H} & 2.410285 & -6.760989 & -2.899877\end{array}$

$\mathrm{H} \quad-0.071031-5.603331 \quad-3.666642$ 


\section{Optimized Cartesian Coordinates and Zero Point Vibrational Energy Corrected Absolute} Energy in Hartree of $Z \mathrm{PhB}$ (Calculated with $\left.\mathrm{CPCM}\left(\mathrm{CH}_{2} \mathrm{Cl}_{2}\right) \mathrm{M06}-2 \mathrm{X} / 6-31+\mathrm{G}(\mathrm{d}, \mathrm{p})\right)$ :

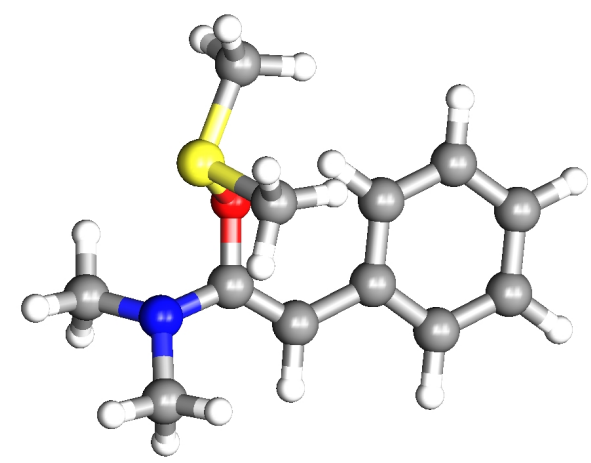

$\mathrm{E}=-995.484669$ a.u. (0 imaginary frequencies)

$\begin{array}{lrlllllll}\mathrm{C} & 0.901017 & 1.021489 & 2.293084 & \mathrm{H} & 2.883404 & 0.559501 & -2.748823 \\ \mathrm{C} & 1.521320 & 1.179966 & 0.938011 & \mathrm{H} & 3.434994 & 1.477739 & -1.330076 \\ \mathrm{C} & 0.858645 & 1.246504 & -0.217805 & \mathrm{H} & 2.845803 & -0.196539 & -1.139518 \\ \mathrm{O} & -0.551403 & 1.294587 & -0.172182 & \mathrm{H} & 1.706013 & 3.218296 & -2.073897 \\ \mathrm{~S} & -1.277143 & 0.237131 & -1.218895 & \mathrm{H} & 0.004755 & 2.707369 & -2.181864 \\ \mathrm{C} & -0.682570 & -1.343034 & -0.615450 & \mathrm{H} & 1.171277 & 2.137777 & -3.391354 \\ \mathrm{~N} & 1.330952 & 1.199639 & -1.525566 & \mathrm{C} & 1.712817 & 0.965044 & 3.432292 \\ \mathrm{C} & 2.704285 & 0.737671 & -1.686913 & \mathrm{C} & 1.133367 & 0.817004 & 4.698120 \\ \mathrm{C} & 1.036614 & 2.388234 & -2.337484 & \mathrm{C} & -0.257883 & 0.725409 & 4.824741 \\ \mathrm{C} & -2.898575 & 0.337769 & -0.465623 & \mathrm{C} & -1.069683 & 0.781855 & 3.685533 \\ \mathrm{H} & 2.603120 & 1.256929 & 0.877473 & \mathrm{C} & -0.490233 & 0.929894 & 2.419705 \\ \mathrm{H} & -1.269737 & -2.117813 & -1.112893 & \mathrm{H} & 2.795011 & 1.036291 & 3.333799 \\ \mathrm{H} & 0.364691 & -1.424045 & -0.909341 & \mathrm{H} & 1.764832 & 0.773098 & 5.584261 \\ \mathrm{H} & -0.803191 & -1.385224 & 0.467920 & \mathrm{H} & -0.708613 & 0.610256 & 5.809375 \\ \mathrm{H} & -3.538956 & -0.373754 & -0.990037 & \mathrm{H} & -2.151878 & 0.710607 & 3.784026 \\ \mathrm{H} & -3.259106 & 1.353661 & -0.627945 & \mathrm{H} & -1.121698 & 0.973801 & 1.533564 \\ \mathrm{H} & -2.821469 & 0.104427 & 0.596332 & & & & \end{array}$




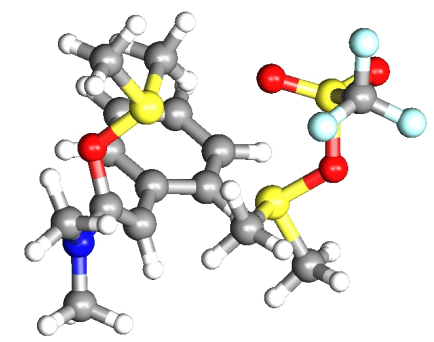

$\mathrm{E}=-2434.271931$ a.u. (0 imaginary frequencies)

\begin{tabular}{|c|c|c|c|c|c|c|c|}
\hline C & 3.637574 & -1.810537 & 0.640556 & $\mathrm{H}$ & 2.966651 & 0.325394 & -1.859529 \\
\hline $\mathrm{C}$ & 2.856047 & -1.351212 & -0.431969 & $\mathrm{H}$ & -0.092999 & 1.116226 & 3.774405 \\
\hline $\mathrm{C}$ & 2.118760 & -2.291784 & -1.165411 & $\mathrm{H}$ & 1.698328 & 1.077413 & 3.969788 \\
\hline $\mathrm{C}$ & 2.111937 & -3.637957 & -0.805097 & $\mathrm{H}$ & 0.890304 & 2.469204 & 3.143491 \\
\hline $\mathrm{C}$ & 2.859625 & -4.073054 & 0.289350 & $\mathrm{H}$ & 0.016317 & -1.280656 & 2.838082 \\
\hline $\mathrm{C}$ & 3.634405 & -3.155512 & 1.000130 & $\mathrm{H}$ & 1.818039 & -1.335290 & 2.957129 \\
\hline $\mathrm{C}$ & 2.843785 & 0.076738 & -0.809693 & $\mathrm{H}$ & 0.981761 & -1.799439 & 1.420206 \\
\hline $\mathrm{C}$ & 2.862702 & 1.099429 & 0.081026 & $\mathrm{H}$ & 3.304533 & 4.356493 & 0.530282 \\
\hline $\mathrm{O}$ & 2.567521 & 0.847855 & 1.447094 & $\mathrm{H}$ & 1.651199 & 3.745945 & 0.320539 \\
\hline $\mathrm{S}$ & 0.986767 & 0.550686 & 1.765314 & $\mathrm{H}$ & 2.650236 & 3.227607 & 1.712379 \\
\hline C & 0.868048 & 1.398209 & 3.339194 & $\mathrm{H}$ & 3.037979 & 2.773244 & -2.208119 \\
\hline $\mathrm{N}$ & 3.237447 & 2.386665 & -0.122355 & $\mathrm{H}$ & 4.545730 & 1.970917 & -1.698768 \\
\hline $\mathrm{C}$ & 2.663970 & 3.483454 & 0.654045 & $\mathrm{H}$ & 4.289554 & 3.694453 & -1.345719 \\
\hline $\mathrm{C}$ & 3.803178 & 2.722068 & -1.421070 & $\mathrm{H}$ & 0.610809 & 0.225339 & -3.595995 \\
\hline $\mathrm{C}$ & 0.961820 & -1.160546 & 2.303371 & $\mathrm{H}$ & -0.469574 & -1.204098 & -3.431944 \\
\hline S & -0.499350 & 0.179380 & -1.524592 & $\mathrm{H}$ & -1.169947 & 0.423739 & -3.791633 \\
\hline $\mathrm{C}$ & -0.550779 & 1.967298 & -1.440720 & $\mathrm{H}$ & 0.450621 & 2.317245 & -1.705671 \\
\hline $\mathrm{O}$ & -2.130194 & -0.160283 & -1.411331 & $\mathrm{H}$ & -1.296809 & 2.342304 & -2.143071 \\
\hline S & -2.753938 & -0.622122 & 0.062495 & $\mathrm{H}$ & -0.783918 & 2.240852 & -0.409944 \\
\hline $\mathrm{O}$ & -1.759805 & -0.306098 & 1.060954 & $\mathrm{H}$ & 1.553583 & -1.974377 & -2.037227 \\
\hline $\mathrm{C}$ & -0.376313 & -0.127569 & -3.286791 & $\mathrm{H}$ & 1.526068 & -4.345841 & -1.382720 \\
\hline $\mathrm{C}$ & -4.080969 & 0.695003 & 0.082876 & $\mathrm{H}$ & 2.854368 & -5.120474 & 0.572616 \\
\hline $\mathrm{F}$ & -4.770400 & 0.550986 & 1.193746 & $\mathrm{H}$ & 4.243089 & -3.487828 & 1.835281 \\
\hline $\mathrm{F}$ & -3.484681 & 1.872606 & 0.054694 & $\mathrm{H}$ & 4.251901 & -1.107039 & 1.194777 \\
\hline & -4.848954 & 0.537451 & -0.971941 & $\mathrm{O}$ & -3.359838 & -1.908349 & -0.091140 \\
\hline
\end{tabular}




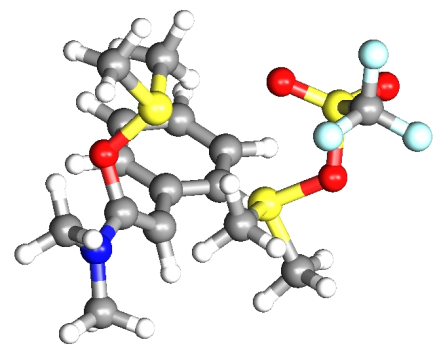

$\mathrm{E}=-2434.269990$ a.u. (1 imaginary frequency)

$\begin{array}{lllllllll}\text { C } & 9.797027 & -6.544626 & 5.624645 & \text { H } & 8.929191 & -4.527431 & 3.163050 \\ \text { C } & 8.882746 & -6.179167 & 4.622774 & \text { H } & 6.117727 & -3.702153 & 8.927496 \\ \text { C } & 8.146925 & -7.194975 & 3.996808 & \text { H } & 7.916834 & -3.657910 & 9.057103 \\ \text { C } & 8.275609 & -8.524659 & 4.394254 & \text { H } & 7.019240 & -2.354260 & 8.176607 \\ \text { C } & 9.156563 & -8.866409 & 5.418992 & \text { H } & 6.357394 & -6.164234 & 8.143216 \\ \text { C } & 9.928071 & -7.871965 & 6.021734 & \text { H } & 8.163303 & -6.120351 & 8.133231 \\ \text { C } & 8.740525 & -4.761685 & 4.205254 & \text { H } & 7.246070 & -6.707777 & 6.685445 \\ \text { C } & 8.876947 & -3.721886 & 5.101945 & \text { H } & 10.020667 & -0.767799 & 5.754293 \\ \text { O } & 8.672763 & -3.959609 & 6.473374 & \text { H } & 8.262988 & -0.699263 & 5.479983 \\ \text { S } & 7.134784 & -4.346992 & 6.917414 & \text { H } & 8.941251 & -1.714530 & 6.783586 \\ \text { C } & 7.051650 & -3.410577 & 8.440763 & \text { H } & 8.934697 & -2.116073 & 2.755402 \\ \text { N } & 9.228914 & -2.461994 & 4.834744 & \text { H } & 10.476694 & -2.894800 & 3.200132 \\ \text { C } & 9.095597 & -1.345414 & 5.774032 & \text { H } & 10.212408 & -1.168059 & 3.538109 \\ \text { C } & 9.738553 & -2.147373 & 3.499620 & \text { H } & 7.180445 & -4.530292 & 1.698398 \\ \text { C } & 7.255683 & -6.025353 & 7.535273 & \text { H } & 6.023615 & -5.907327 & 1.754099 \\ \text { S } & 6.001059 & -4.670222 & 3.766892 & \text { H } & 5.417088 & -4.232175 & 1.504899 \\ \text { C } & 5.860543 & -2.885467 & 3.958996 & \text { H } & 6.846636 & -2.443678 & 3.808049 \\ \text { O } & 4.283196 & -4.963031 & 3.669264 & \text { H } & 5.148891 & -2.516100 & 3.220037 \\ \text { S } & 3.532844 & -5.476645 & 5.016815 & \text { H } & 5.509981 & -2.695340 & 4.975324 \\ \text { O } & 4.416914 & -5.243515 & 6.140397 & \text { H } & 7.474527 & -6.958820 & 3.178051 \\ \text { C } & 6.178291 & -4.851840 & 1.979263 & \text { H } & 7.689075 & -9.291573 & 3.898999 \\ \text { C } & 2.221005 & -4.154504 & 5.031254 & \text { H } & 9.254373 & -9.900345 & 5.732947 \\ \text { F } & 1.488180 & -4.320067 & 6.114436 & \text { H } & 10.638088 & -8.130097 & 6.801172 \\ \text { F } & 2.819389 & -2.974637 & 5.059045 & \text { H } & 10.411312 & -5.782529 & 6.095006 \\ \text { F } & 1.480408 & -4.266608 & 3.948098 & \text { O } & 2.908210 & -6.742040 & 4.761907\end{array}$


Optimized Cartesian Coordinates and Zero Point Vibrational Energy Corrected Absolute Energy in Hartree of $Z \mathrm{PhCT}$ rif (Calculated with $\mathrm{CPCM}\left(\mathrm{CH}_{2} \mathrm{Cl}_{2}\right) \mathrm{M06-2X/6-31+G(d,p)):}$

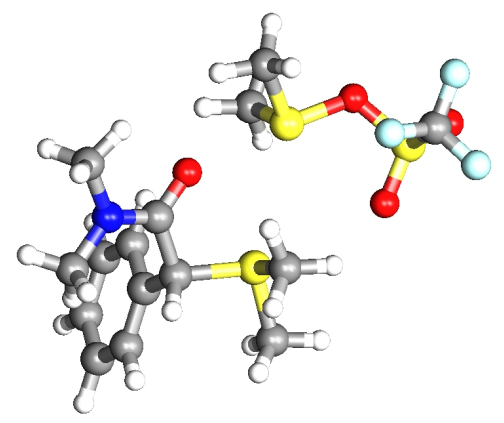

$\mathrm{E}=-2434.358244$ a.u. (0 imaginary frequencies)

$\begin{array}{lrrrrrrrr}\mathrm{C} & 2.885578 & -1.810476 & 0.138283 & & \mathrm{H} & 2.168761 & 0.658367 & -2.121970 \\ \mathrm{C} & 2.784926 & -1.133864 & -1.081762 & \mathrm{H} & -1.372700 & 1.478885 & 3.836236 \\ \mathrm{C} & 3.452707 & -1.622785 & -2.208398 & \mathrm{H} & -0.000143 & 2.074149 & 2.816733 \\ \mathrm{C} & 4.216562 & -2.783949 & -2.114185 & \mathrm{H} & -1.670550 & 2.630515 & 2.471716 \\ \mathrm{C} & 4.313729 & -3.458683 & -0.897763 & \mathrm{H} & -0.926132 & -1.038619 & 3.507286 \\ \mathrm{C} & 3.649641 & -2.970825 & 0.227922 & \mathrm{H} & 0.615322 & -0.588793 & 2.675598 \\ \mathrm{C} & 1.947588 & 0.119967 & -1.195099 & \mathrm{H} & -0.499179 & -1.778969 & 1.913886 \\ \mathrm{C} & 2.074592 & 1.040833 & 0.030403 & \mathrm{H} & 4.179443 & 2.451145 & 1.796382 \\ \mathrm{O} & 1.200832 & 0.998199 & 0.918796 & \mathrm{H} & 3.496302 & 3.756004 & 0.792800 \\ \mathrm{~S} & -1.230408 & 0.452877 & 1.689444 & \mathrm{H} & 2.422996 & 2.760509 & 1.810873 \\ \mathrm{C} & -1.059224 & 1.809268 & 2.845964 & \mathrm{H} & 3.904253 & 2.619033 & -1.726016 \\ \mathrm{~N} & 3.144801 & 1.826456 & 0.080785 & \mathrm{H} & 4.335156 & 0.908467 & -1.415558 \\ \mathrm{C} & 3.318907 & 2.756364 & 1.195972 & \mathrm{H} & 5.107240 & 2.191000 & -0.489564 \\ \mathrm{C} & 4.173130 & 1.884899 & -0.960912 & \mathrm{H} & 0.551733 & -0.728068 & -3.641624 \\ \mathrm{C} & -0.423781 & -0.896396 & 2.549872 & \mathrm{H} & 0.655385 & -2.231885 & -2.651045 \\ \mathrm{~S} & 0.165739 & -0.355055 & -1.288118 & \mathrm{H} & -0.938001 & -1.513476 & -3.014113 \\ \mathrm{C} & -0.607435 & 1.201166 & -1.784699 & \mathrm{H} & -0.161321 & 1.551817 & -2.716027 \\ \mathrm{O} & -3.044863 & -0.482210 & -0.394607 & \mathrm{H} & -1.672915 & 1.002101 & -1.906254 \\ \mathrm{~S} & -3.840313 & -0.272780 & 0.789487 & \mathrm{H} & -0.448331 & 1.915728 & -0.975950 \\ \mathrm{O} & -2.815644 & 0.041307 & 2.052443 & \mathrm{H} & 3.385837 & -1.093665 & -3.155886 \\ \mathrm{C} & 0.114911 & -1.301014 & -2.823026 & \mathrm{H} & 4.737103 & -3.157208 & -2.989682 \\ \mathrm{C} & -4.596172 & 1.428181 & 0.686355 & \mathrm{H} & 4.910809 & -4.361816 & -0.826058 \\ \mathrm{~F} & -5.289221 & 1.661567 & 1.778782 & \mathrm{H} & 3.729523 & -3.490105 & 1.177006 \\ \mathrm{~F} & -3.597265 & 2.296056 & 0.579935 & \mathrm{H} & 2.376708 & -1.426419 & 1.018428 \\ \mathrm{~F} & -5.363765 & 1.483805 & -0.380353 & -4.808126 & -1.196669 & 1.295690\end{array}$




\section{Optimized Cartesian Coordinates and Zero Point Vibrational Energy Corrected Absolute} Energy in Hartree of $\mathrm{EPhA}$ (Calculated with $\mathrm{CPCM}\left(\mathrm{CH}_{2} \mathrm{Cl}_{2}\right) \mathrm{M06-2X/6-31+G(d,p)):}$

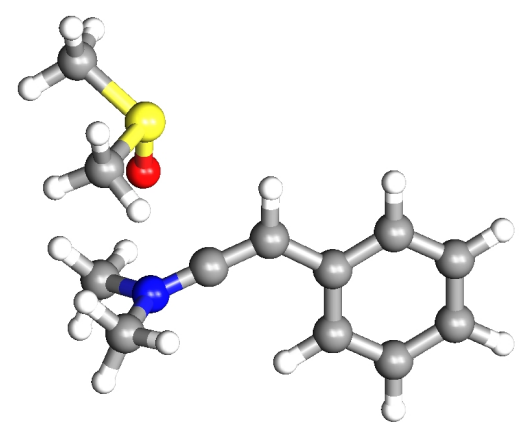

$\mathrm{E}=-995.461778$ a.u. (0 imaginary frequencies)

$\begin{array}{lllllllll}\mathrm{C} & -0.000624 & -1.905284 & 1.481335 & \mathrm{H} & 0.237323 & 3.225911 & 0.091645 \\ \mathrm{C} & -0.118416 & -0.861880 & 0.448642 & \mathrm{H} & -0.016464 & 3.229554 & 1.870432 \\ \mathrm{C} & -0.454140 & 0.380546 & 0.643027 & \mathrm{H} & 1.221250 & 2.156361 & 1.137466 \\ \mathrm{~N} & -0.774388 & 1.573013 & 0.838323 & \mathrm{H} & -2.424114 & 2.626667 & 1.580714 \\ \mathrm{C} & -2.171744 & 2.034443 & 0.701152 & \mathrm{H} & -2.824465 & 1.168825 & 0.615792 \\ \mathrm{C} & 0.249321 & 2.625353 & 1.002991 & \mathrm{H} & -2.221894 & 2.645909 & -0.201412 \\ \mathrm{O} & -0.189984 & 1.232575 & -1.823984 & \mathrm{C} & 0.403184 & -3.185906 & 1.090144 \\ \mathrm{~S} & 0.668846 & 1.203869 & -3.093912 & \mathrm{C} & 0.527803 & -4.198107 & 2.039763 \\ \mathrm{C} & -0.386072 & 1.860591 & -4.402953 & \mathrm{C} & 0.250354 & -3.935837 & 3.380019 \\ \mathrm{C} & 1.780839 & 2.617888 & -2.947586 & \mathrm{C} & -0.154251 & -2.657224 & 3.772784 \\ \mathrm{H} & 0.085400 & -1.099109 & -0.595604 & \mathrm{C} & -0.279961 & -1.644380 & 2.829854 \\ \mathrm{H} & 1.187466 & 3.507854 & -2.725799 & \mathrm{H} & 0.619244 & -3.387438 & 0.045122 \\ \mathrm{H} & 2.469720 & 2.403517 & -2.129640 & \mathrm{H} & 0.841598 & -5.189556 & 1.730546 \\ \mathrm{H} & 2.335073 & 2.737519 & -3.880790 & \mathrm{H} & 0.348265 & -4.723855 & 4.119548 \\ \mathrm{H} & 0.207487 & 1.991809 & -5.310010 & \mathrm{H} & -0.372003 & -2.451077 & 4.815543 \\ \mathrm{H} & -0.809059 & 2.809950 & -4.067427 & \mathrm{H} & -0.596286 & -0.651997 & 3.140906 \\ \mathrm{H} & -1.176327 & 1.129944 & -4.577305 & & & & \end{array}$




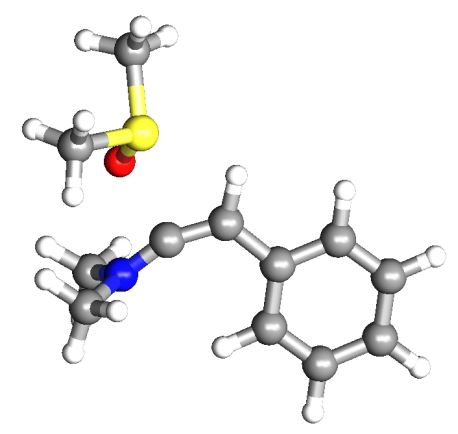

$\mathrm{E}=-995.458870$ a.u. (1 imaginary frequency)

$\begin{array}{llll}\mathrm{C} & 4.769857 & -4.736541 & -0.047713 \\ \mathrm{C} & 4.611228 & -3.729716 & -1.117235 \\ \mathrm{C} & 4.362722 & -2.463332 & -0.887915 \\ \mathrm{~N} & 4.062201 & -1.437668 & -0.201744 \\ \mathrm{C} & 2.655395 & -1.078476 & 0.052826 \\ \mathrm{C} & 5.067252 & -0.397056 & 0.066205 \\ \mathrm{O} & 4.420635 & -1.597649 & -2.824827 \\ \mathrm{~S} & 5.631952 & -1.856021 & -3.756121 \\ \mathrm{C} & 4.910227 & -2.030752 & -5.395276 \\ \mathrm{C} & 6.398494 & -0.240895 & -3.959028 \\ \mathrm{H} & 4.697796 & -4.059062 & -2.146943 \\ \mathrm{H} & 5.628266 & 0.476009 & -4.249910 \\ \mathrm{H} & 6.838533 & 0.031624 & -2.999329 \\ \mathrm{H} & 7.178598 & -0.315056 & -4.719048 \\ \mathrm{H} & 5.717082 & -2.096952 & -6.127643 \\ \mathrm{H} & 4.268659 & -1.169278 & -5.589370 \\ \mathrm{H} & 4.329354 & -2.953161 & -5.393667\end{array}$

$\begin{array}{llll}\mathrm{H} & 4.930967 & 0.409627 & -0.658449 \\ \mathrm{H} & 4.915129 & -0.022060 & 1.079005 \\ \mathrm{H} & 6.061880 & -0.828493 & -0.034117 \\ \mathrm{H} & 2.558396 & -0.800476 & 1.103212 \\ \mathrm{H} & 2.024127 & -1.935526 & -0.173246 \\ \mathrm{H} & 2.388979 & -0.231658 & -0.583789 \\ \mathrm{C} & 4.950371 & -6.071122 & -0.428649 \\ \mathrm{C} & 5.097396 & -7.063861 & 0.538124 \\ \mathrm{C} & 5.068946 & -6.730048 & 1.890714 \\ \mathrm{C} & 4.892602 & -5.398819 & 2.275152 \\ \mathrm{C} & 4.742944 & -4.405358 & 1.314064 \\ \mathrm{H} & 4.973499 & -6.330547 & -1.483209 \\ \mathrm{H} & 5.235294 & -8.095821 & 0.232347 \\ \mathrm{H} & 5.185659 & -7.501755 & 2.644564 \\ \mathrm{H} & 4.872632 & -5.134647 & 3.327454 \\ \mathrm{H} & 4.607884 & -3.373237 & 1.625202\end{array}$


Optimized Cartesian Coordinates and Zero Point Vibrational Energy Corrected Absolute Energy in Hartree of EPhB (Calculated with $\left.\mathrm{CPCM}\left(\mathrm{CH}_{2} \mathrm{Cl}_{2}\right) \mathrm{M06}-2 \mathrm{X} / 6-31+\mathrm{G}(\mathrm{d}, \mathrm{p})\right)$ :

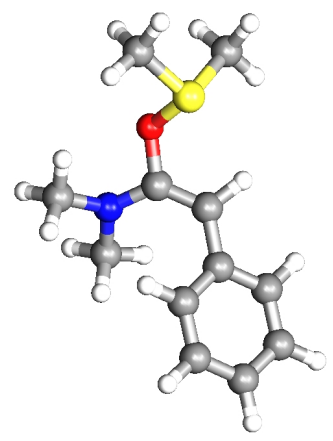

$\mathrm{E}=-995.479509$ a.u. (0 imaginary frequencies)

$\begin{array}{lrrr}\mathrm{C} & -0.160480 & -1.459587 & 0.794016 \\ \mathrm{C} & -0.205936 & -0.547711 & -0.359168 \\ \mathrm{C} & -0.368287 & 0.786647 & -0.280209 \\ \mathrm{~N} & -0.883610 & 1.563852 & 0.702170 \\ \mathrm{C} & -1.890622 & 1.042349 & 1.612013 \\ \mathrm{C} & -0.229504 & 2.795853 & 1.126950 \\ \mathrm{O} & -0.109417 & 1.608347 & -1.426135 \\ \mathrm{~S} & 1.131354 & 1.177462 & -2.393377 \\ \mathrm{C} & 0.244293 & 0.830809 & -3.911492 \\ \mathrm{C} & 1.792683 & 2.810983 & -2.715883 \\ \mathrm{H} & -0.123661 & -1.002045 & -1.343572 \\ \mathrm{H} & 0.979781 & 3.473842 & -3.013143 \\ \mathrm{H} & 2.273596 & 3.145813 & -1.796951 \\ \mathrm{H} & 2.535545 & 2.706528 & -3.509074 \\ \mathrm{H} & 0.988908 & 0.687409 & -4.696685 \\ \mathrm{H} & -0.420041 & 1.665850 & -4.135838 \\ \mathrm{H} & -0.313671 & -0.092684 & -3.753794\end{array}$

$\begin{array}{cccc}\mathrm{H} & -0.987016 & 3.559761 & 1.319150 \\ \mathrm{H} & 0.344842 & 2.628134 & 2.047244 \\ \mathrm{H} & 0.439625 & 3.156362 & 0.347316 \\ \mathrm{H} & -1.455047 & 0.721909 & 2.566694 \\ \mathrm{H} & -2.393015 & 0.191402 & 1.151173 \\ \mathrm{H} & -2.619523 & 1.834180 & 1.807084 \\ \mathrm{C} & -0.630230 & -2.774660 & 0.659584 \\ \mathrm{C} & -0.571792 & -3.665784 & 1.728286 \\ \mathrm{C} & -0.038574 & -3.260577 & 2.952325 \\ \mathrm{C} & 0.446515 & -1.959781 & 3.093455 \\ \mathrm{C} & 0.392603 & -1.070028 & 2.023781 \\ \mathrm{H} & -1.048266 & -3.096165 & -0.290807 \\ \mathrm{H} & -0.944462 & -4.677922 & 1.604811 \\ \mathrm{H} & 0.008004 & -3.954411 & 3.785495 \\ \mathrm{H} & 0.878999 & -1.639015 & 4.036289 \\ \mathrm{H} & 0.801052 & -0.068157 & 2.133031\end{array}$




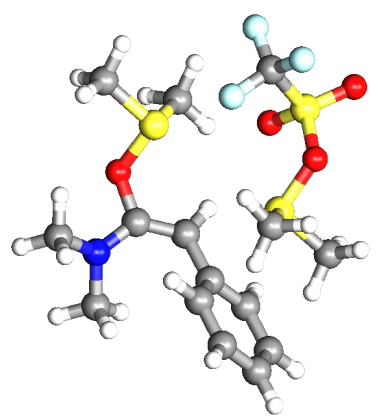

$E=-2434.282355$ a.u. (0 imaginary frequencies)

\begin{tabular}{lrrr}
$\mathrm{C}$ & 1.362174 & 1.722802 & -3.848819 \\
$\mathrm{C}$ & 1.557921 & 1.490764 & -2.486586 \\
$\mathrm{C}$ & 2.118675 & 0.280973 & -2.034188 \\
$\mathrm{C}$ & 2.432556 & -0.703501 & -2.985235 \\
$\mathrm{C}$ & 2.234878 & -0.470850 & -4.344516 \\
$\mathrm{C}$ & 1.704397 & 0.745227 & -4.783876 \\
$\mathrm{C}$ & 2.349018 & 0.030179 & -0.603705 \\
$\mathrm{C}$ & 2.800253 & 0.964871 & 0.261050 \\
$\mathrm{~N}$ & 3.461797 & 2.120556 & 0.035607 \\
$\mathrm{C}$ & 4.292557 & 2.289304 & -1.146880 \\
$\mathrm{O}$ & 2.735629 & 0.719875 & 1.663187 \\
$\mathrm{~S}$ & 1.342017 & 0.087650 & 2.239347 \\
$\mathrm{C}$ & 1.848873 & -1.575360 & 2.674180 \\
$\mathrm{C}$ & 1.360411 & 0.885207 & 3.844239 \\
$\mathrm{C}$ & 3.155131 & 3.344630 & 0.768242 \\
$\mathrm{O}$ & -0.507213 & -1.880605 & 0.810841 \\
$\mathrm{~S}$ & -1.929700 & -1.913282 & 0.579958 \\
$\mathrm{O}$ & -2.710957 & -3.108178 & 0.487867 \\
$\mathrm{O}$ & -2.252299 & -1.048007 & -0.794127 \\
$\mathrm{~S}$ & -1.027022 & -0.158795 & -1.519287 \\
$\mathrm{C}$ & -2.023975 & 1.269595 & -1.943196 \\
$\mathrm{C}$ & -0.952535 & -1.046763 & -3.070352 \\
$\mathrm{C}$ & -2.748285 & -0.738293 & 1.773580 \\
$\mathrm{~F}$ & -2.188730 & 0.451887 & 1.611096 \\
\hline
\end{tabular}

$\begin{array}{lrrr}\mathrm{F} & -2.533535 & -1.179643 & 2.996060 \\ \mathrm{H} & 2.205101 & -0.985865 & -0.245645 \\ \mathrm{H} & 0.549876 & 0.440736 & 4.426076 \\ \mathrm{H} & 2.328645 & 0.719899 & 4.317140 \\ \mathrm{H} & 1.165155 & 1.944454 & 3.679521 \\ \mathrm{H} & 1.042157 & -2.003835 & 3.273683 \\ \mathrm{H} & 2.782962 & -1.523598 & 3.234695 \\ \mathrm{H} & 1.958438 & -2.144041 & 1.751319 \\ \mathrm{H} & 4.086539 & 3.842225 & 1.048642 \\ \mathrm{H} & 2.564692 & 4.023619 & 0.140843 \\ \mathrm{H} & 2.595038 & 3.114765 & 1.672626 \\ \mathrm{H} & 3.758475 & 2.805185 & -1.953913 \\ \mathrm{H} & 4.622125 & 1.313863 & -1.505810 \\ \mathrm{H} & 5.164735 & 2.885062 & -0.865598 \\ \mathrm{H} & -0.343466 & -0.434466 & -3.744145 \\ \mathrm{H} & -0.458170 & -1.998684 & -2.874952 \\ \mathrm{H} & -1.967340 & -1.175750 & -3.448329 \\ \mathrm{H} & -1.408641 & 1.879751 & -2.608698 \\ \mathrm{H} & -2.934554 & 0.933041 & -2.439009 \\ \mathrm{H} & -2.230466 & 1.807572 & -1.017960 \\ \mathrm{H} & 2.852480 & -1.648577 & -2.651837 \\ \mathrm{H} & 2.495479 & -1.240704 & -5.064023 \\ \mathrm{H} & 1.554392 & 0.926127 & -5.842988 \\ \mathrm{H} & 0.941351 & 2.668184 & -4.178146 \\ \mathrm{H} & 1.293311 & 2.260101 & -1.762544\end{array}$




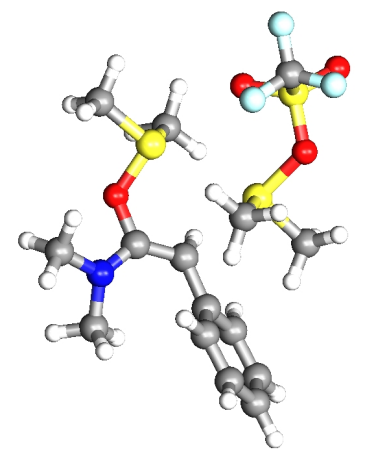

$\mathrm{E}=-2434.268064$ a.u. (1 imaginary frequency)

\begin{tabular}{llll} 
C & 7.033104 & -1.772633 & 3.815156 \\
C & 7.505580 & -2.983106 & 4.350293 \\
C & 7.932199 & -3.983157 & 3.461715 \\
C & 7.888085 & -3.780591 & 2.085090 \\
C & 7.414127 & -2.575138 & 1.565725 \\
C & 6.989856 & -1.572391 & 2.437002 \\
C & 7.492476 & -3.276597 & 5.797531 \\
C & 7.928447 & -2.355971 & 6.761491 \\
O & 7.902547 & -2.683649 & 8.117991 \\
S & 6.609076 & -3.442679 & 8.799230 \\
C & 6.855726 & -2.837574 & 10.465837 \\
N & 8.499380 & -1.171384 & 6.607119 \\
C & 8.501231 & -0.170999 & 7.683867 \\
C & 9.373161 & -0.846776 & 5.473811 \\
C & 7.201563 & -5.130524 & 8.924772 \\
S & 4.931416 & -4.006322 & 6.148592 \\
C & 4.812443 & -4.630552 & 4.463365 \\
C & 4.314193 & -2.326007 & 5.959754 \\
O & 3.303145 & -4.686146 & 6.560854 \\
S & 3.021844 & -5.221734 & 8.039982 \\
O & 4.200156 & -5.023864 & 8.862633 \\
C & 1.775200 & -3.943567 & 8.549707 \\
F & 0.745220 & -3.980044 & 7.725873 \\
F & 1.386662 & -4.208038 & 9.783496 \\
\hline
\end{tabular}

\begin{tabular}{llll} 
H & 7.691924 & -4.313217 & 6.065840 \\
$\mathrm{H}$ & 6.143039 & -3.374993 & 11.095464 \\
$\mathrm{H}$ & 7.884875 & -3.035298 & 10.767701 \\
$\mathrm{H}$ & 6.624743 & -1.773072 & 10.460304 \\
$\mathrm{H}$ & 6.556920 & -5.632396 & 9.649427 \\
$\mathrm{H}$ & 8.242824 & -5.109949 & 9.248909 \\
$\mathrm{H}$ & 7.087133 & -5.602698 & 7.948965 \\
$\mathrm{H}$ & 9.356220 & -0.323354 & 8.347474 \\
$\mathrm{H}$ & 8.576662 & 0.809779 & 7.213775 \\
$\mathrm{H}$ & 7.573803 & -0.219898 & 8.250677 \\
$\mathrm{H}$ & 8.901002 & -0.113244 & 4.816208 \\
$\mathrm{H}$ & 9.620159 & -1.744312 & 4.912994 \\
$\mathrm{H}$ & 10.287313 & -0.420207 & 5.892905 \\
$\mathrm{H}$ & 5.342749 & -3.940179 & 3.805527 \\
$\mathrm{H}$ & 5.265623 & -5.621423 & 4.449264 \\
$\mathrm{H}$ & 3.750636 & -4.667733 & 4.216966 \\
$\mathrm{H}$ & 4.948248 & -1.820199 & 5.231441 \\
$\mathrm{H}$ & 3.288231 & -2.394517 & 5.595349 \\
$\mathrm{H}$ & 4.363206 & -1.837405 & 6.933337 \\
$\mathrm{H}$ & 8.296817 & -4.927606 & 3.857099 \\
$\mathrm{H}$ & 8.224340 & -4.567052 & 1.417178 \\
$\mathrm{H}$ & 7.377190 & -2.418047 & 0.493052 \\
$\mathrm{H}$ & 6.622278 & -0.628925 & 2.046542 \\
$\mathrm{H}$ & 6.715547 & -0.968772 & 4.475443 \\
\hline
\end{tabular}


Optimized Cartesian Coordinates and Zero Point Vibrational Energy Corrected Absolute Energy in Hartree of $E P h C T r i f\left(C a l c u l a t e d ~ w i t h ~ C P C M\left(C H_{2} \mathrm{Cl}_{2}\right) \mathrm{M06-2X/6-31+G(d,p)):}\right.$

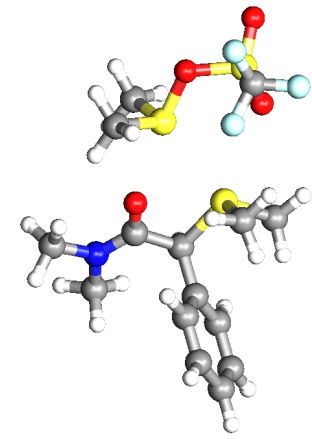

$\mathrm{E}=-2434.359414$ a.u. (0 imaginary frequencies)

\begin{tabular}{lrrr} 
C & 1.407674 & 2.145683 & -2.753153 \\
C & 1.930578 & 0.848271 & -2.811250 \\
C & 2.356129 & 0.325891 & -4.036644 \\
C & 2.246867 & 1.089756 & -5.196725 \\
C & 1.716048 & 2.377466 & -5.136603 \\
C & 1.299820 & 2.906084 & -3.914201 \\
C & 2.059760 & 0.022387 & -1.559616 \\
C & 2.399808 & 0.853315 & -0.313446 \\
O & 1.545164 & 0.972308 & 0.585451 \\
S & -0.169539 & -0.149703 & 2.209490 \\
C & -0.051303 & 1.336034 & 3.201374 \\
N & 3.588060 & 1.448163 & -0.282880 \\
C & 3.915118 & 2.357262 & 0.815440 \\
C & 4.607620 & 1.334780 & -1.326104 \\
C & 1.240031 & -1.109799 & 2.764759 \\
S & 0.525398 & -0.929600 & -1.117286 \\
C & 0.329028 & -1.988040 & -2.564212 \\
C & -0.828648 & 0.236651 & -1.361461 \\
O & -2.039957 & -1.966258 & 0.919943 \\
S & -2.513540 & -1.771576 & 2.267300 \\
O & -1.356820 & -0.928191 & 3.102105 \\
C & -3.810041 & -0.432108 & 2.241076 \\
\hline & -4.814022 & -0.840929 & 1.494899 \\
F & -4.206006 & -0.187370 & 3.470500 \\
\hline
\end{tabular}

$\begin{array}{cccc}\mathrm{H} & 2.789889 & -0.782412 & -1.696672 \\ \mathrm{H} & 0.104259 & 1.065361 & 4.245623 \\ \mathrm{H} & 0.799370 & 1.880976 & 2.786169 \\ \mathrm{H} & -0.973323 & 1.898268 & 3.053546 \\ \mathrm{H} & 1.173211 & -1.227022 & 3.846965 \\ \mathrm{H} & 2.124904 & -0.544568 & 2.467982 \\ \mathrm{H} & 1.198290 & -2.069011 & 2.247780 \\ \mathrm{H} & 4.654665 & 1.892967 & 1.473104 \\ \mathrm{H} & 4.332381 & 3.274563 & 0.394833 \\ \mathrm{H} & 3.014136 & 2.588774 & 1.378082 \\ \mathrm{H} & 4.513812 & 2.156696 & -2.042448 \\ \mathrm{H} & 4.538701 & 0.385290 & -1.853380 \\ \mathrm{H} & 5.585286 & 1.384793 & -0.845020 \\ \mathrm{H} & 0.311655 & -1.383092 & -3.471766 \\ \mathrm{H} & 1.153314 & -2.701432 & -2.572582 \\ \mathrm{H} & -0.616157 & -2.515676 & -2.429848 \\ \mathrm{H} & -0.817754 & 0.608772 & -2.386529 \\ \mathrm{H} & -1.743187 & -0.320744 & -1.150914 \\ \mathrm{H} & -0.698559 & 1.044046 & -0.640672 \\ \mathrm{H} & 2.791105 & -0.669651 & -4.083143 \\ \mathrm{H} & 2.585312 & 0.681432 & -6.143016 \\ \mathrm{H} & 1.636965 & 2.973318 & -6.039906 \\ \mathrm{H} & 0.896605 & 3.911738 & -3.862175 \\ \mathrm{H} & 1.093180 & 2.569992 & -1.801621 \\ \mathrm{O} & -2.933291 & -2.820068 & 3.146024\end{array}$




\section{Optimized Cartesian Coordinates and Zero Point Vibrational Energy Corrected Absolute} Energy in Hartree of $Z \mathrm{PhB}^{\prime}$ (Calculated with $\mathrm{CPCM}\left(\mathrm{CH}_{2} \mathrm{Cl}_{2}\right) \mathrm{M06-2X/6-31+G(d,p)):}$

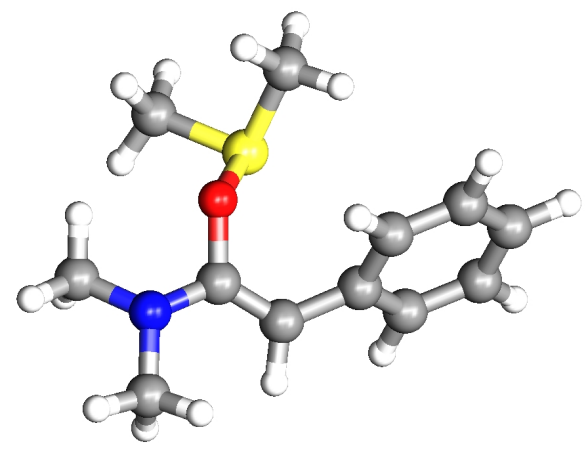

$\mathrm{E}=-995.482155$ a.u. (0 imaginary frequencies)

$\begin{array}{lllllllll}\mathrm{C} & -0.137565 & 2.744423 & -0.463931 & \mathrm{H} & -0.482370 & -0.613274 & 3.873415 \\ \mathrm{~S} & -0.831230 & 1.343525 & 0.409630 & \mathrm{H} & 1.175203 & -0.407842 & 4.490018 \\ \mathrm{C} & -1.525775 & 2.153351 & 1.851070 & \mathrm{H} & 0.484555 & 0.830981 & 3.440235 \\ \mathrm{O} & 0.562381 & 0.747220 & 1.034870 & \mathrm{H} & 2.028353 & -2.364560 & 3.664214 \\ \mathrm{C} & 0.606607 & -0.662711 & 1.231641 & \mathrm{H} & 2.071586 & -2.700477 & 1.918830 \\ \mathrm{~N} & 1.090752 & -0.940214 & 2.480233 & \mathrm{H} & 0.570070 & -2.971386 & 2.845216 \\ \mathrm{C} & 1.454261 & -2.326359 & 2.737535 & \mathrm{C} & -0.897618 & -1.792487 & -1.925035 \\ \mathrm{C} & 0.297871 & -1.521399 & 0.242129 & \mathrm{C} & -1.081072 & -1.483248 & -3.269690 \\ \mathrm{C} & 0.111517 & -1.167242 & -1.176740 & \mathrm{C} & -0.257600 & -0.545598 & -3.895842 \\ \mathrm{C} & 0.523253 & -0.240578 & 3.631819 & \mathrm{C} & 0.761262 & 0.068330 & -3.168194 \\ \mathrm{H} & 0.195724 & -2.566058 & 0.515142 & \mathrm{C} & 0.949067 & -0.244236 & -1.822110 \\ \mathrm{H} & 0.534262 & 3.279888 & 0.207891 & \mathrm{H} & -1.543655 & -2.520579 & -1.442040 \\ \mathrm{H} & 0.381616 & 2.353272 & -1.338170 & \mathrm{H} & -1.869617 & -1.975081 & -3.830710 \\ \mathrm{H} & -0.978807 & 3.368772 & -0.770766 & \mathrm{H} & -0.401231 & -0.306205 & -4.944492 \\ \mathrm{H} & -2.379378 & 2.739276 & 1.501638 & \mathrm{H} & 1.423500 & 0.781104 & -3.650845 \\ \mathrm{H} & -0.769299 & 2.791996 & 2.308689 & \mathrm{H} & 1.769209 & 0.209890 & -1.270688 \\ \mathrm{H} & -1.871219 & 1.374948 & 2.531596 & & & & \end{array}$




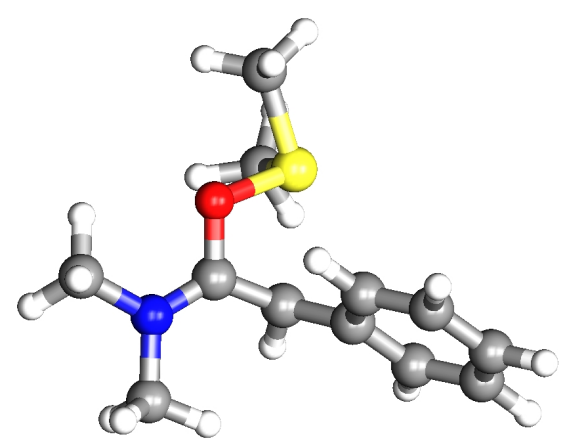

$\mathrm{E}=-995.449092$ a.u. (1 imaginary frequency)

$\begin{array}{lrllllllll}\mathrm{C} & 0.296689 & -2.449924 & -2.007053 & \mathrm{H} & 2.279601 & -6.565561 & 1.501697 \\ \mathrm{~S} & -0.282474 & -4.146603 & -2.379533 & \mathrm{H} & 3.456799 & -6.074574 & 0.255065 \\ \mathrm{C} & -1.720500 & -4.138675 & -1.297079 & \mathrm{H} & 2.097830 & -4.994155 & 0.669909 \\ \mathrm{O} & 1.097601 & -4.704693 & -1.401782 & \mathrm{H} & 2.595890 & -8.503676 & -0.300316 \\ \mathrm{C} & 0.909007 & -6.031985 & -1.350524 & \mathrm{H} & 1.153359 & -8.545803 & -1.346763 \\ \mathrm{~N} & 1.580949 & -6.691765 & -0.455705 & \mathrm{H} & 0.971401 & -8.488785 & 0.433929 \\ \mathrm{C} & 1.567927 & -8.157817 & -0.418957 & \mathrm{C} & -0.600887 & -7.489324 & -4.490714 \\ \mathrm{C} & -0.097025 & -6.491292 & -2.275081 & \mathrm{C} & -0.306247 & -7.716190 & -5.831937 \\ \mathrm{C} & 0.278239 & -6.758711 & -3.672386 & \mathrm{C} & 0.874082 & -7.223513 & -6.391320 \\ \mathrm{C} & 2.409160 & -6.030509 & 0.560219 & \mathrm{C} & 1.755683 & -6.498155 & -5.590096 \\ \mathrm{H} & -0.814217 & -7.193177 & -1.851979 & \mathrm{C} & 1.462680 & -6.263629 & -4.248513 \\ \mathrm{H} & 0.342935 & -2.271314 & -0.931787 & \mathrm{H} & -1.519886 & -7.882908 & -4.064423 \\ \mathrm{H} & 1.278356 & -2.325784 & -2.464299 & \mathrm{H} & -1.000328 & -8.286252 & -6.442182 \\ \mathrm{H} & -0.420477 & -1.779343 & -2.484731 & \mathrm{H} & 1.104461 & -7.406104 & -7.435725 \\ \mathrm{H} & -2.192070 & -3.157283 & -1.372608 & \mathrm{H} & 2.680659 & -6.112875 & -6.008363 \\ \mathrm{H} & -1.382735 & -4.312229 & -0.272667 & \mathrm{H} & 2.172460 & -5.700687 & -3.644879 \\ \mathrm{H} & -2.380521 & -4.939209 & -1.626201 & & & & \end{array}$




\section{Optimized Cartesian Coordinates and Zero Point Vibrational Energy Corrected Absolute} Energy in Hartree of $Z \mathrm{PhD}$ (Calculated with $\mathrm{CPCM}\left(\mathrm{CH}_{2} \mathrm{Cl}_{2}\right) \mathrm{M06-2X/6-31+G(d,p)):}$

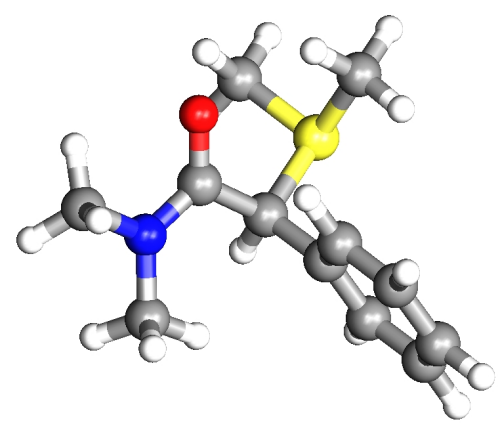

$\mathrm{E}=-995.552867$ a.u. (0 imaginary frequencies)

$\begin{array}{lrrr}\mathrm{C} & 1.054132 & -0.109297 & -1.234561 \\ \mathrm{C} & -0.223651 & -0.504009 & -0.826135 \\ \mathrm{C} & -1.017220 & -1.283262 & -1.673864 \\ \mathrm{C} & -0.534358 & -1.668254 & -2.921462 \\ \mathrm{C} & 0.741774 & -1.276223 & -3.327031 \\ \mathrm{C} & 1.534059 & -0.497658 & -2.484038 \\ \mathrm{C} & -0.763773 & -0.089584 & 0.526274 \\ \mathrm{C} & 0.339798 & 0.136472 & 1.573467 \\ \mathrm{~N} & 0.886457 & -0.964275 & 2.113833 \\ \mathrm{C} & 1.978488 & -0.797598 & 3.068863 \\ \mathrm{O} & 0.693496 & 1.281590 & 1.861585 \\ \mathrm{C} & 0.627798 & -2.342816 & 1.711864 \\ \mathrm{~S} & -1.834678 & 1.391812 & 0.279598 \\ \mathrm{C} & -2.241453 & 1.950647 & 1.947812 \\ \mathrm{C} & -0.750396 & 2.694725 & -0.341646 \\ \mathrm{H} & -1.494365 & -0.823569 & 0.881396 \\ \mathrm{H} & 0.045236 & 2.882594 & 0.375719\end{array}$

$$
\begin{array}{rrrr}
\mathrm{H} & -0.362844 & 2.354336 & -1.302601 \\
\mathrm{H} & -1.391208 & 3.564614 & -0.492462 \\
\mathrm{H} & -2.948054 & 2.772582 & 1.830647 \\
\mathrm{H} & -1.332957 & 2.259652 & 2.461000 \\
\mathrm{H} & -2.729180 & 1.117286 & 2.455302 \\
\mathrm{H} & 1.907268 & -1.588793 & 3.817386 \\
\mathrm{H} & 2.944924 & -0.871621 & 2.559148 \\
\mathrm{H} & 1.896228 & 0.174644 & 3.549672 \\
\mathrm{H} & 1.541343 & -2.772156 & 1.288600 \\
\mathrm{H} & -0.156926 & -2.412318 & 0.962788 \\
\mathrm{H} & 0.333993 & -2.925656 & 2.588220 \\
\mathrm{H} & -2.008893 & -1.594081 & -1.354571 \\
\mathrm{H} & -1.151467 & -2.278579 & -3.572315 \\
\mathrm{H} & 1.119467 & -1.581436 & -4.297432 \\
\mathrm{H} & 2.527474 & -0.192983 & -2.795798 \\
\mathrm{H} & 1.675649 & 0.497400 & -0.579881
\end{array}
$$

H $\quad 0.045236 \quad 2.882594 \quad 0.375719$ 


\section{Optimized Cartesian Coordinates and Zero Point Vibrational Energy Corrected Absolute} Energy in Hartree of $Z \mathrm{PhB}^{\prime \prime}$ (Calculated with $\mathrm{CPCM}\left(\mathrm{CH}_{2} \mathrm{Cl}_{2}\right) \mathrm{M06-2X/6-31+G(d,p)):}$

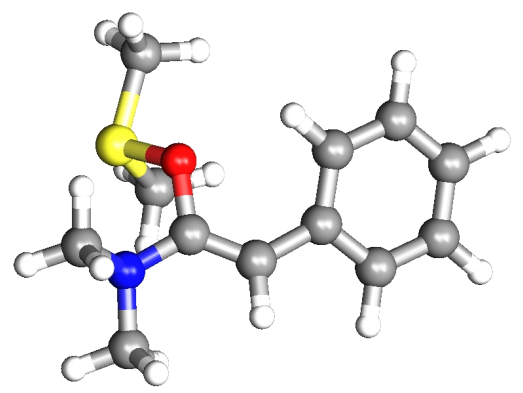

$\mathrm{E}=-995.484625$ a.u. (0 imaginary frequencies)

$\begin{array}{lllllllll}\mathrm{C} & 1.916145 & -1.226187 & 2.630671 & \mathrm{H} & 3.646526 & -1.669361 & 0.603889 \\ \mathrm{~N} & 2.072220 & -0.480401 & 1.387840 & \mathrm{H} & 3.481090 & -0.056095 & -0.128373 \\ \mathrm{C} & 3.414894 & -0.615329 & 0.805592 & \mathrm{H} & 4.145104 & -0.213339 & 1.509806 \\ \mathrm{C} & 1.016153 & -0.542225 & 0.495061 & \mathrm{H} & 2.715077 & -0.926214 & 3.310852 \\ \mathrm{O} & 1.081656 & 0.475742 & -0.470731 & \mathrm{H} & 1.982698 & -2.311975 & 2.471487 \\ \mathrm{C} & -0.054749 & -1.350019 & 0.508662 & \mathrm{H} & 0.952728 & -0.988244 & 3.086042 \\ \mathrm{C} & -1.300206 & -1.230052 & -0.269062 & \mathrm{C} & -1.393236 & -0.611537 & -1.527041 \\ \mathrm{~S} & 1.360132 & 1.979565 & 0.175341 & \mathrm{C} & -2.619505 & -0.524627 & -2.183243 \\ \mathrm{C} & -0.084835 & 2.183276 & 1.219221 & \mathrm{C} & -3.771777 & -1.059296 & -1.607704 \\ \mathrm{C} & 0.886560 & 2.884553 & -1.295286 & \mathrm{C} & -3.687583 & -1.699102 & -0.370920 \\ \mathrm{H} & -0.016318 & -2.166154 & 1.224176 & \mathrm{C} & -2.464127 & -1.787487 & 0.286687 \\ \mathrm{H} & -0.139136 & 3.241201 & 1.484783 & \mathrm{H} & -0.504718 & -0.218343 & -2.005934 \\ \mathrm{H} & -0.968668 & 1.860732 & 0.665302 & \mathrm{H} & -2.671010 & -0.045899 & -3.156384 \\ \mathrm{H} & 0.074813 & 1.581788 & 2.114843 & \mathrm{H} & -4.723876 & -0.989683 & -2.124074 \\ \mathrm{H} & 1.609625 & 2.626503 & -2.069800 & \mathrm{H} & -4.574592 & -2.131015 & 0.081774 \\ \mathrm{H} & 0.962493 & 3.946097 & -1.053274 & \mathrm{H} & -2.404633 & -2.287391 & 1.249931 \\ \mathrm{H} & -0.128200 & 2.608558 & -1.581802 & & & & \end{array}$




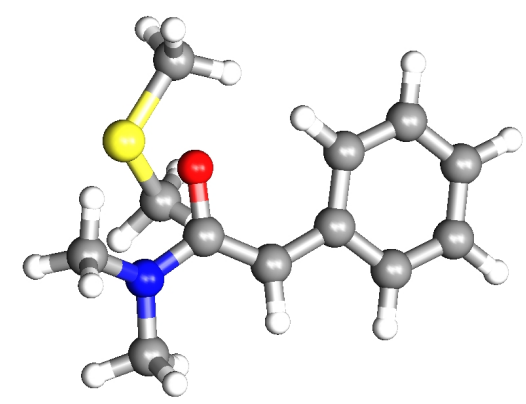

$\mathrm{E}=-995.463387$ a.u. (1 imaginary frequency)

$\begin{array}{lllllllll}\mathrm{C} & -1.022953 & -1.417418 & 2.159612 & \mathrm{H} & 1.108630 & -1.629452 & 0.537851 \\ \mathrm{~N} & -0.780348 & -0.762046 & 0.881467 & \mathrm{H} & 0.708530 & -0.235634 & -0.499374 \\ \mathrm{C} & 0.628449 & -0.644059 & 0.505851 & \mathrm{H} & 1.137374 & 0.022147 & 1.206372 \\ \mathrm{C} & -1.741040 & -0.738845 & -0.091476 & \mathrm{H} & -0.259534 & -1.077640 & 2.861019 \\ \mathrm{O} & -1.532213 & -0.013085 & -1.131136 & \mathrm{H} & -0.963073 & -2.510372 & 2.081319 \\ \mathrm{C} & -2.983643 & -1.387526 & 0.067715 & \mathrm{H} & -1.998134 & -1.129738 & 2.557388 \\ \mathrm{C} & -4.159023 & -1.288749 & -0.751264 & \mathrm{C} & -4.234728 & -0.626573 & -2.000038 \\ \mathrm{~S} & -1.381843 & 2.022316 & -0.387284 & \mathrm{C} & -5.447160 & -0.533714 & -2.664732 \\ \mathrm{C} & -2.669259 & 1.908142 & 0.833126 & \mathrm{C} & -6.603325 & -1.101385 & -2.117078 \\ \mathrm{C} & -2.259524 & 2.602221 & -1.850203 & \mathrm{C} & -6.540865 & -1.787057 & -0.902398 \\ \mathrm{H} & -3.047817 & -2.117741 & 0.870532 & \mathrm{C} & -5.330657 & -1.891708 & -0.233187 \\ \mathrm{H} & -3.441204 & 2.654894 & 0.641418 & \mathrm{H} & -3.336157 & -0.220761 & -2.445260 \\ \mathrm{H} & -3.125302 & 0.899983 & 0.788679 & \mathrm{H} & -5.495266 & -0.030357 & -3.624668 \\ \mathrm{H} & -2.218097 & 2.045704 & 1.815415 & \mathrm{H} & -7.547108 & -1.024265 & -2.647410 \\ \mathrm{H} & -1.685803 & 2.273311 & -2.717470 & \mathrm{H} & -7.432720 & -2.241089 & -0.484331 \\ \mathrm{H} & -2.297878 & 3.692499 & -1.820301 & \mathrm{H} & -5.278272 & -2.424090 & 0.712331 \\ \mathrm{H} & -3.262844 & 2.178797 & -1.874871 & & & & \end{array}$




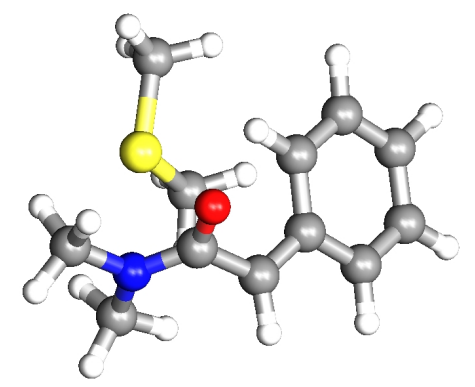

$\mathrm{E}=-995.497880$ a.u. (0 imaginary frequencies)

$\begin{array}{ccccccccc}\mathrm{C} & 1.780998 & -0.682673 & 2.341643 & \mathrm{H} & 4.272624 & -1.180642 & 1.557909 \\ \mathrm{~N} & 2.251047 & -1.168012 & 1.051998 & \mathrm{H} & 3.919482 & -1.427256 & -0.173906 \\ \mathrm{C} & 3.639284 & -0.876292 & 0.721515 & \mathrm{H} & 3.760745 & 0.198273 & 0.549536 \\ \mathrm{C} & 1.452017 & -1.707011 & 0.127413 & \mathrm{H} & 1.953014 & 0.396497 & 2.410997 \\ \mathrm{O} & 1.778817 & -2.058067 & -1.007227 & \mathrm{H} & 2.319715 & -1.187706 & 3.147042 \\ \mathrm{C} & 0.024520 & -1.974894 & 0.519733 & \mathrm{H} & 0.711365 & -0.864192 & 2.464502 \\ \mathrm{C} & -1.054085 & -1.457954 & -0.156798 & \mathrm{C} & -0.894792 & -0.583222 & -1.288745 \\ \mathrm{~S} & 0.632815 & 1.831745 & 0.287774 & \mathrm{C} & -2.012010 & -0.031207 & -1.874178 \\ \mathrm{C} & -0.926939 & 1.970789 & 1.203270 & \mathrm{C} & -3.283935 & -0.317597 & -1.350051 \\ \mathrm{C} & 0.258349 & 2.960845 & -1.079908 & \mathrm{C} & -3.469811 & -1.177153 & -0.251475 \\ \mathrm{H} & -0.152250 & -2.669749 & 1.340904 & \mathrm{C} & -2.371528 & -1.758170 & 0.333576 \\ \mathrm{H} & -1.089334 & 3.000270 & 1.528689 & \mathrm{H} & 0.097216 & -0.408553 & -1.687098 \\ \mathrm{H} & -1.767980 & 1.646018 & 0.583059 & \mathrm{H} & -1.920595 & 0.612773 & -2.741037 \\ \mathrm{H} & -0.859284 & 1.326386 & 2.082016 & \mathrm{H} & -4.155779 & 0.130094 & -1.818190 \\ \mathrm{H} & 1.120640 & 2.967878 & -1.747629 & \mathrm{H} & -4.468345 & -1.379544 & 0.116540 \\ \mathrm{H} & 0.085126 & 3.970455 & -0.702406 & \mathrm{H} & -2.476258 & -2.431284 & 1.179314 \\ \mathrm{H} & -0.621722 & 2.617350 & -1.629687 & & & & \end{array}$




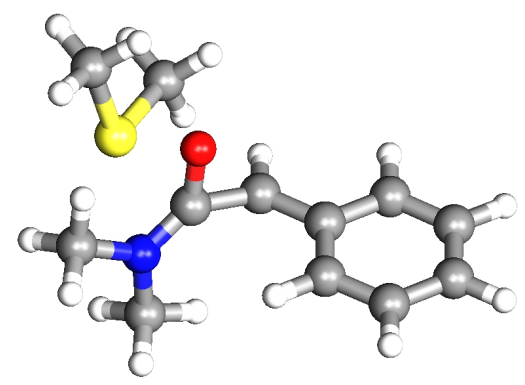

$\mathrm{E}=-995.494116$ a.u. (1 imaginary frequency)

$\begin{array}{lllllllll}\mathrm{C} & 5.886128 & 0.485057 & 1.868745 & \mathrm{H} & 0.102689 & -1.208221 & -0.316296 \\ \mathrm{C} & 5.324938 & 0.626591 & 0.609531 & \mathrm{H} & 1.781606 & -1.321802 & -0.911632 \\ \mathrm{C} & 3.922835 & 0.722649 & 0.476507 & \mathrm{H} & 2.013965 & 0.758198 & 1.515731 \\ \mathrm{C} & 3.093096 & 0.683078 & 1.620499 & \mathrm{H} & 3.039272 & 0.521590 & 3.757840 \\ \mathrm{C} & 3.664651 & 0.550800 & 2.873105 & \mathrm{H} & 5.499048 & 0.344385 & 3.978859 \\ \mathrm{C} & 5.056840 & 0.451435 & 2.993473 & \mathrm{H} & 6.961224 & 0.405399 & 1.981568 \\ \mathrm{C} & 3.372481 & 0.830080 & -0.829143 & \mathrm{H} & 5.951966 & 0.658245 & -0.276124 \\ \mathrm{C} & 1.991408 & 1.165262 & -1.100056 & \mathrm{~S} & 2.456114 & 0.341166 & -4.226993 \\ \mathrm{~N} & 0.855967 & 0.540293 & -1.182528 & \mathrm{C} & 2.179718 & 2.121088 & -4.449183 \\ \mathrm{C} & 0.788728 & -0.918281 & -1.114112 & \mathrm{C} & 4.105061 & 0.212563 & -4.971445 \\ \mathrm{O} & 2.308312 & 2.377626 & -1.180481 & \mathrm{H} & 4.070972 & 0.506617 & -6.022296 \\ \mathrm{C} & -0.372712 & 1.280930 & -1.468307 & \mathrm{H} & 4.820738 & 0.840962 & -4.436179 \\ \mathrm{H} & 4.003960 & 0.761711 & -1.711700 & \mathrm{H} & 4.418132 & -0.829911 & -4.900549 \\ \mathrm{H} & -1.104251 & 1.070199 & -0.686471 & \mathrm{H} & 1.195197 & 2.359627 & -4.041785 \\ \mathrm{H} & -0.151144 & 2.347640 & -1.488641 & \mathrm{H} & 2.195109 & 2.378089 & -5.510316 \\ \mathrm{H} & -0.765281 & 0.959161 & -2.435786 & \mathrm{H} & 2.934948 & 2.699418 & -3.912069 \\ \mathrm{H} & 0.431652 & -1.301914 & -2.072344 & & & & \end{array}$


Optimized Cartesian Coordinates and Zero Point Vibrational Energy Corrected Absolute Energy in Hartree of $Z \mathrm{PhF}$ (Calculated with $\mathrm{CPCM}\left(\mathrm{CH}_{2} \mathrm{Cl}_{2}\right) \mathrm{M06}-2 \mathrm{X} / 6-31+\mathrm{G}(\mathrm{d}, \mathrm{p})$ ):

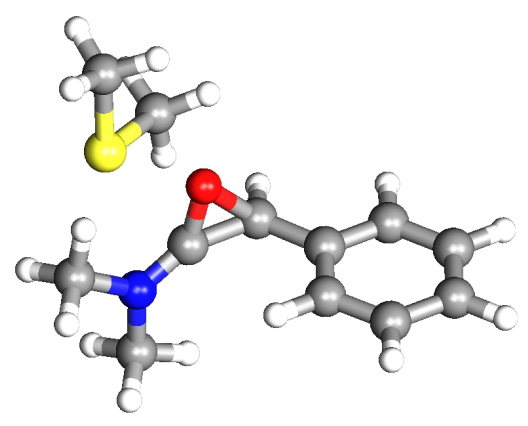

$\mathrm{E}=-995.498065$ a.u. (0 imaginary frequencies)

$\begin{array}{lrrrrrrrr}\mathrm{C} & 3.024532 & -0.206416 & 2.926261 & \mathrm{H} & -2.995115 & -2.118488 & 0.696317 \\ \mathrm{C} & 2.441053 & -0.122784 & 1.664291 & \mathrm{H} & -1.298911 & -2.337182 & 0.173157 \\ \mathrm{C} & 1.055610 & 0.019495 & 1.549687 & \mathrm{H} & -0.825663 & 0.187756 & 2.609832 \\ \mathrm{C} & 0.252889 & 0.080710 & 2.695331 & \mathrm{H} & 0.222669 & 0.051236 & 4.841670 \\ \mathrm{C} & 0.841467 & 0.004655 & 3.952139 & \mathrm{H} & 2.681409 & -0.205827 & 5.049947 \\ \mathrm{C} & 2.226027 & -0.140635 & 4.067070 & \mathrm{H} & 4.099530 & -0.318731 & 3.017503 \\ \mathrm{C} & 0.471463 & 0.105659 & 0.198930 & \mathrm{H} & 3.058112 & -0.168036 & 0.771758 \\ \mathrm{C} & -0.942118 & 0.108807 & -0.046613 & \mathrm{~S} & -0.575675 & -0.067423 & -3.215186 \\ \mathrm{~N} & -2.099202 & -0.423362 & -0.129556 & \mathrm{C} & -0.406442 & 1.733775 & -3.348691 \\ \mathrm{C} & -2.257536 & -1.881317 & -0.071028 & \mathrm{C} & 1.037261 & -0.565097 & -3.880015 \\ \mathrm{O} & -0.473809 & 1.311187 & -0.070361 & \mathrm{H} & 1.127685 & -0.258010 & -4.923748 \\ \mathrm{C} & -3.278622 & 0.402286 & -0.420341 & \mathrm{H} & 1.849451 & -0.125905 & -3.296107 \\ \mathrm{H} & 1.106673 & -0.074830 & -0.664363 & \mathrm{H} & 1.097352 & -1.652456 & -3.818957 \\ \mathrm{H} & -4.027385 & 0.221925 & 0.351289 & \mathrm{H} & -1.341164 & 2.179248 & -3.003612 \\ \mathrm{H} & -2.989940 & 1.452546 & -0.426550 & \mathrm{H} & -0.234763 & 2.023078 & -4.387458 \\ \mathrm{H} & -3.668082 & 0.111753 & -1.398043 & \mathrm{H} & 0.412531 & 2.092157 & -2.720239 \\ \mathrm{H} & -2.601988 & -2.230390 & -1.046173 & & & & \end{array}$




\section{Optimized Cartesian Coordinates and Zero Point Vibrational Energy Corrected Absolute} Energy in Hartree of $Z \mathrm{PhF}^{\prime}$ (Calculated with $\mathrm{CPCM}\left(\mathrm{CH}_{2} \mathrm{Cl}_{2}\right) \mathrm{M06-2X/6-31+G(d,p)):}$

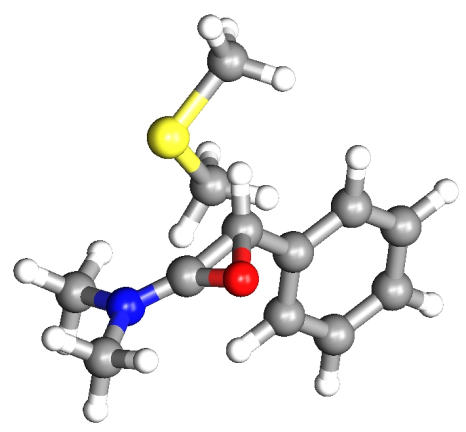

$\mathrm{E}=-995.499642$ a.u. (0 imaginary frequencies)

$\begin{array}{lrrrrrrrr}\mathrm{C} & 2.258757 & -0.560809 & -1.757544 & \text { H } & -3.920998 & -0.939769 & -0.689859 \\ \mathrm{C} & 1.720220 & -0.630040 & -3.044002 & \mathrm{H} & -2.606601 & -1.049319 & -1.887944 \\ \mathrm{C} & 0.470089 & -0.073466 & -3.315475 & \mathrm{H} & 0.181924 & -2.855584 & 0.179153 \\ \mathrm{C} & -0.244236 & 0.557084 & -2.299803 & \mathrm{H} & -0.262504 & -2.143408 & -1.390735 \\ \mathrm{C} & 0.295132 & 0.626051 & -1.011424 & \mathrm{H} & -1.030359 & -3.665169 & -0.843929 \\ \mathrm{C} & 1.547045 & 0.064481 & -0.738796 & \mathrm{H} & 1.999962 & 1.997189 & 3.662273 \\ \mathrm{C} & -0.486357 & 1.306242 & 0.035242 & \mathrm{H} & 1.064213 & 3.285934 & 2.847707 \\ \mathrm{C} & 0.040819 & 1.609471 & 1.334250 & \mathrm{H} & 0.391093 & 2.465030 & 4.286978 \\ \mathrm{~N} & 0.371966 & 1.328229 & 2.535901 & \mathrm{H} & -0.495451 & 0.078510 & 3.967492 \\ \mathrm{C} & 0.146653 & -0.013463 & 3.090749 & \mathrm{H} & 1.111472 & -0.436096 & 3.374540 \\ \mathrm{O} & 0.127271 & 2.651341 & 0.582909 & \mathrm{H} & -0.336486 & -0.632762 & 2.335774 \\ \mathrm{C} & 1.002024 & 2.344763 & 3.391682 & \mathrm{H} & 1.962597 & 0.103252 & 0.265387 \\ \mathrm{~S} & -1.919088 & -1.741477 & 0.327417 & \mathrm{H} & 3.227445 & -1.001626 & -1.547955 \\ \mathrm{C} & -0.636796 & -2.697654 & -0.525363 & \mathrm{H} & 2.274426 & -1.124222 & -3.835264 \\ \mathrm{C} & -3.088865 & -1.549650 & -1.044257 & \mathrm{H} & 0.052391 & -0.129931 & -4.314948 \\ \mathrm{H} & -1.557778 & 1.423535 & -0.097851 & \mathrm{H} & -1.215377 & 0.999258 & -2.504598 \\ \mathrm{H} & -3.465478 & -2.523831 & -1.362061 & & & & \end{array}$


Optimized Cartesian Coordinates and Zero Point Vibrational Energy Corrected Absolute Energy in Hartree of $\mathrm{TS}_{Z \mathrm{PhF}^{\prime}-\mathrm{G}}\left(\mathrm{Calculated}\right.$ with $\left.\mathrm{CPCM}\left(\mathrm{CH}_{2} \mathrm{Cl}_{2}\right) \mathrm{M06}-2 \mathrm{X} / 6-31+\mathrm{G}(\mathrm{d}, \mathrm{p})\right)$ :

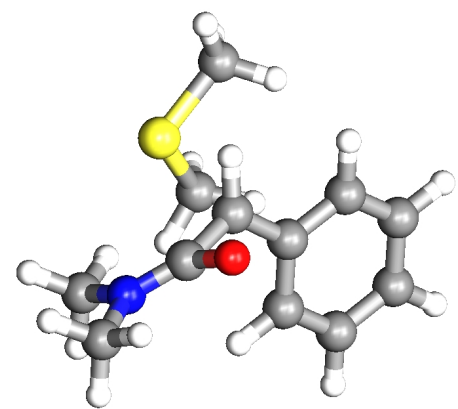

$\mathrm{E}=-995.497438 \mathrm{a} . \mathrm{u}$. (1 imaginary frequency)

\begin{tabular}{|c|c|c|c|c|c|c|c|}
\hline C & 2.116540 & -0.502784 & -3.885119 & $\mathrm{H}$ & -2.135445 & -1.558498 & -1.245180 \\
\hline $\mathrm{C}$ & 1.330497 & -0.000626 & -2.856249 & $\mathrm{H}$ & -0.773172 & -1.725710 & -2.386492 \\
\hline $\mathrm{C}$ & 1.869212 & 0.121141 & -1.562658 & $\mathrm{H}$ & 2.031151 & -3.160101 & -0.084002 \\
\hline $\mathrm{C}$ & 3.201556 & -0.254970 & -1.311673 & $\mathrm{H}$ & 1.597403 & -2.607785 & -1.722379 \\
\hline $\mathrm{C}$ & 3.980038 & -0.755303 & -2.345522 & $\mathrm{H}$ & 0.894377 & -4.115872 & -1.064813 \\
\hline $\mathrm{C}$ & 3.436775 & -0.879881 & -3.628020 & $\mathrm{H}$ & 3.519286 & 1.764720 & 3.009662 \\
\hline $\mathrm{C}$ & 1.026580 & 0.610679 & -0.504231 & $\mathrm{H}$ & 2.367461 & 2.924329 & 2.289020 \\
\hline $\mathrm{C}$ & 1.554228 & 1.220314 & 0.701761 & $\mathrm{H}$ & 1.880923 & 1.940945 & 3.698596 \\
\hline $\mathrm{N}$ & 1.977641 & 0.905024 & 1.887321 & $\mathrm{H}$ & 1.404556 & -0.540727 & 3.282967 \\
\hline $\mathrm{C}$ & 2.012697 & -0.471492 & 2.379566 & $\mathrm{H}$ & 3.046408 & -0.738675 & 2.607969 \\
\hline $\mathrm{O}$ & 1.493867 & 2.321708 & 0.098321 & $\mathrm{H}$ & 1.612439 & -1.139830 & 1.619909 \\
\hline $\mathrm{C}$ & 2.469810 & 1.957915 & 2.780420 & $\mathrm{H}$ & 3.617847 & -0.164556 & -0.311838 \\
\hline $\mathrm{S}$ & -0.136512 & -2.160500 & -0.094180 & $\mathrm{H}$ & 5.005578 & -1.052702 & -2.156876 \\
\hline $\mathrm{C}$ & 1.227393 & -3.105399 & -0.820796 & $\mathrm{H}$ & 4.048651 & -1.273806 & -4.433063 \\
\hline $\mathrm{C}$ & -1.264462 & -2.158570 & -1.511018 & $\mathrm{H}$ & 1.706241 & -0.597534 & -4.884340 \\
\hline$H$ & -0.043015 & 0.719732 & -0.647660 & $\mathrm{H}$ & 0.307589 & 0.311856 & -3.045853 \\
\hline & & & & & & & \\
\hline
\end{tabular}


Optimized Cartesian Coordinates and Zero Point Vibrational Energy Corrected Absolute Energy in Hartree of $Z$ PhG (Calculated with $\mathrm{CPCM}\left(\mathrm{CH}_{2} \mathrm{Cl}_{2}\right) \mathrm{M06-2X/6-31+G(d,p)):}$

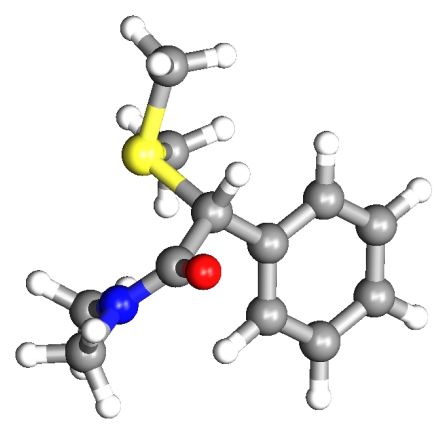

$\mathrm{E}=-995.538944$ a.u. (0 imaginary frequencies)

$\begin{array}{lrrrrrrrr}\mathrm{C} & 1.055247 & -0.465447 & -3.303856 & \mathrm{H} & -3.656347 & -0.236747 & 0.213316 \\ \mathrm{C} & 0.069056 & -0.211850 & -2.356176 & \mathrm{H} & -2.999404 & -1.056963 & -1.249931 \\ \mathrm{C} & 0.416499 & 0.137303 & -1.044700 & \mathrm{H} & 0.340781 & -2.531870 & 0.072446 \\ \mathrm{C} & 1.766797 & 0.271417 & -0.707507 & \mathrm{H} & -0.852385 & -2.465711 & -1.265282 \\ \mathrm{C} & 2.753673 & 0.027781 & -1.661749 & \mathrm{H} & -1.196063 & -3.431918 & 0.214734 \\ \mathrm{C} & 2.401461 & -0.351619 & -2.955081 & \mathrm{H} & 1.484076 & 2.415100 & 3.149219 \\ \mathrm{C} & -0.689002 & 0.402573 & -0.052980 & \mathrm{H} & -0.266185 & 2.736062 & 3.203349 \\ \mathrm{C} & -0.334806 & 1.393663 & 1.073856 & \mathrm{H} & 0.436306 & 1.453222 & 4.224419 \\ \mathrm{~N} & 0.263010 & 0.974698 & 2.205655 & \mathrm{H} & 0.249570 & -0.899479 & 3.179823 \\ \mathrm{C} & 0.844305 & -0.338990 & 2.452672 & \mathrm{H} & 1.854843 & -0.204541 & 2.848346 \\ \mathrm{O} & -0.638343 & 2.565242 & 0.864440 & \mathrm{H} & 0.944627 & -0.916472 & 1.533944 \\ \mathrm{C} & 0.494583 & 1.959964 & 3.259646 & \mathrm{H} & 2.062377 & 0.592382 & 0.286645 \\ \mathrm{~S} & -1.510011 & -1.144140 & 0.645603 & \mathrm{H} & 3.798007 & 0.142042 & -1.391256 \\ \mathrm{C} & -0.718582 & -2.537325 & -0.186462 & \mathrm{H} & 3.172237 & -0.541320 & -3.694785 \\ \mathrm{C} & -3.121249 & -1.106808 & -0.167789 & \mathrm{H} & 0.772662 & -0.736838 & -4.315447 \\ \mathrm{H} & -1.519536 & 0.889762 & -0.569122 & \mathrm{H} & -0.977690 & -0.270301 & -2.644581 \\ \mathrm{H} & -3.640201 & -2.020388 & 0.124703 & & & & \end{array}$




\section{References and Comments}

1 Still, W. C.; Kahn, M.; Mitra, A. Rapid Chromatographic Technique for Preparative Separations with Moderate Resolution. J. Org. Chem. 1978, 43, 2923-2925.

2 Pangborn, A. B.; Giardello, M. A.; Grubbs, R. H.; Rosen, R. K.; Timmers, F. J. Safe and Convenient Procedure for Solvent Purification. Organometallics 1996, 15, 1518-1520.

3 Fulmer, G. R., Miller, A. J. M.; Sherden, N. H.; Gottlieb, H. E.; Nudelman, A.; Stoltz, B. M.; Bercaw, J. E.; Goldberg, K. I. NMR Chemical Shifts of Trace Impurities: Common Laboratory Solvents, Organics, and Gases in Deuterated Solvents Relevant to the Organometallic Chemist. Organometallics 2010, 29, 2176-2179.

4 The MIT Department of Chemistry diffractometer was purchased with the help of funding from the National Science Foundation (NSF) under Grant Number CHE-0946721.

5 A modified experimental procedure was applied to avoid over-oxidation to tert-butyl methyl sulfone: Zenzola, M.; Doran, R.; Degennaro, L.; Luisi, R.; Bull, J. A. Transfer of Electrophilic NH Using Convenient Sources of Ammonia: Direct Synthesis of NH Sulfoximines from Sulfoxides. Angew. Chem. Int. Ed. 2016, 55, 7203-7207.

6 A modified experimental procedure was applied: Drabowicz, J.; Dudzinski, B.; Mikolajczyk, M.; Wang, F.; Dehlavi, A.; Goring, J.; Park, M.; Rizzo, C. J.; Polavarapu, P. L.; Biscarini, P.; Wieczorek, M. W.; Wieslaw, R. M. Absolute Configuration, Predominant Conformations, and Vibrational Circular Dichroism Spectra of Enantiomers of $n$-Butyl tert-Butyl Sulfoxide. J. Org. Chem. 2001, 66, 1122-1129.

7 For possible oxidative $\mathrm{C}-\mathrm{C}$ coupling products, see Kaiser, D.; de la Torre, A.; Shaaban, S.; Maulide, N. Metal-Free Formal Oxidative $\mathrm{C}-\mathrm{C}$ Coupling by In Situ Generation of an Enolonium Species. Angew. Chem. Int. Ed. 2017, 56, 5921-5925. 2-Methyl-4-phenyl-1,4-dihydroisoquinolin-3(2H)-one was not detected.

9 1-Methyl-3-phenylindolin-2-one (49.0 mg, 44\%) was separately isolated.

10 1-Methyl-3-phenylindolin-2-one (52.7 mg, 47\%) was separately isolated.

11 The yield of 5-methoxy-1-methyl-3-phenylindolin-2-one was not determined.

12 5-Methoxy-1-methyl-3-phenylindolin-2-one (53.9 mg, 43\%) was separately isolated.

13 2-Benzyl-4-phenyl-1,4-dihydroisoquinolin-3(2H)-one $(96.2 \mathrm{mg}, 61 \%)$ was separately isolated.

14 2-Benzyl-4-phenyl-1,4-dihydroisoquinolin-3(2H)-one $(53.9 \mathrm{mg}, 43 \%)$ was separately isolated.

15 Recovered starting material accounts for most of mass balance.

16 Although the trifluoromethanesulfonate could not be exchanged with bicarbonate during aqueous work-up, NMR-titration of $N, N$-dimethyl amide dimethyl sulfonium trifluoromethanesulfonate 3za with $n$-butylammonium chloride in chloroform- $d$ revealed a 1:2 binding isotherm for the exchange of trifluoromethanesulfonate with chloride. The thermodynamic association constants were determined to $K_{1}=1360 \pm 110 \mathrm{M}^{-1}$ and $\mathrm{K}_{2}=1.16 \pm$ $0.17 \mathrm{M}^{-1}$. The fit to a 1:2 binding isotherm was performed using BindFit available at: supramolecular.org (April 08, 2020). Hibbert, D. B.; Thordarson, P. The Death of the Job Plot, Transparency, Open Science and Online Tools, Uncertainty Estimation Methods and Other Developments in Supramolecular Chemistry Data Analysis. Chem. Commun. 2016, 52, 1279212805.

17 Application of Method A with amide $\mathbf{S 1}$ as substrate gave the corresponding $\alpha$-hydroxy amide instead of $\alpha$-SMe amide $\mathbf{S 4}$.

18 For related in situ IR measurements, see White, K. L.; Mewald, M.; Movassaghi, M. Direct Observation of Intermediates Involved in the Interruption of the Bischler-Napieralski Reaction. J. Org. Chem. 2015, 80, 7403-7411. 
19 A modified experimental procedure was applied: Fenselau, A. H.; Moffatt, J. G. SulfoxideCarbodiimide Reactions. III. ${ }^{1}$ Mechanism of the Oxidation Reaction. J. Am. Chem. Soc. 1966, $88,1762-1765$.

20 (Methylthio)methyl trifluoromethanesulfonate was detected as by-product in NMR-experiments using chloroform- $d$ as solvent.

21 Frisch, M. J.; Trucks, G. W.; Schlegel, H. B.; Scuseria, G. E.; Robb, M. A.; Cheeseman, J. R.; Scalmani, G.; Barone, V.; Mennucci, B.; Petersson, G. A.; Nakatsuji, H.; Caricato, M.; Li, X.; Hratchian, H. P.; Izmaylov, A. F.; Bloino, J.; Zheng, G.; Sonnenberg, J. L.; Hada, M.; Ehara, M.; Toyota, K.; Fukuda, R.; Hasegawa, J.; Ishida, M.; Nakajima, T.; Honda, Y.; Kitao, O.; Nakai, H.; Vreven, T.; Montgomery Jr., J. A.; Peralta, J. E.; Ogliaro, F.; Bearpark, M.; Heyd, J. J.; Brothers, E.; Kudin, K. N.; Staroverov, N.; Keith, T. V.; Kobayashi, R.; Normand, J.; Raghavachari, K.; Rendell, A.; Burant, J. C.; Iyengar, S. S.; Tomasi, J.; Cossi, M.; Rega, N.; Millam, J. M.; Klene, M.; Knox, J. E.; Cross, J. B.; Bakken, V.; Adamo, C.; Jaramillo, J.; Gomperts, R.; Stratmann, R. E.; Yazyev, O.; Austin, A. J.; Cammi, R.; Pomelli, C.; Ochterski, J. W.; Martin, R. L.; Morokuma, K.; Zakrzewski, V. G.; Voth, G. A.; Salvador, P.; Dannenberg, J. J.; Dapprich, S.; Daniels, A. D.; Farkas, O.; Foresman, J. B.; Ortiz, J. V.; Cioslowski, J.; Fox, D. J. Gaussian 09, Revision D.01; Gaussian, Inc.: Wallingford, CT, 2013.

22 Zhao, Y.; Truhlar, D. G. The M06 Suite of Density Functionals for Main Group Thermochemistry, Thermochemical Kinetics, Noncovalent Interactions, Excited States, and Transition Elements: Two New Functionals and Systematic Testing of Four M06-Class Functionals and 12 Other Functionals. Theor. Chem. Acc. 2008, 120, 215-241.

23 (a) Hariharan, P. C.; Pople, J. A. The Influence of Polarization Functions on Molecular Orbital Hydrogenation Energies. Theor. Chim. Acta 1973, 28, 213-222. (b) Hehre, W. J.; Ditchfield, R.; Pople, J. A. Self-Consistent Molecular Orbital Methods. XII. Further Extensions of GaussianType Basis Sets for Use in Molecular Orbital Studies of Organic Molecules. J. Chem. Phys. 1972, 56, 2257-2261. (c) Ditchfield, R.; Hehre, W. J.; Pople, J. A. Self-Consistent MolecularOrbital Methods. IX. An Extended Gaussian-Type Basis for Molecular-Orbital Studies of Organic Molecules. J. Chem. Phys. 1971, 54, 724-728.

24 Tomasi, J.; Mennucci, B.; Cammi, R. Quantum Mechanical Continuum Solvation Models. Chem. Rev. 2005, 105, 2999-3093.

25 Allouche, A.-R. Gabedit-A Graphical User Interface for Computational Chemistry Softwares. J. Comput. Chem. 2011, 32, 174-182.

26 For fragmentative $\alpha$-functionalization of amides, see Gonçalves, C. R.; Lemmerer, M.; Teskey, C. J.; Adler, P.; Kaiser, D.; Maryasin, B.; González, L.; Maulide, N. Unified Approach to the Chemoselective $\alpha$-Functionalization of Amides with Heteroatom Nucleophiles. J. Am. Chem. Soc. 2019, 141, 18437-18443.

27 For transition state $\mathbf{T} \mathbf{S}_{Z \mathbf{P h E}} \mathbf{E}^{\prime} \mathbf{F}$, the reactive conformer $\boldsymbol{Z} \mathbf{P h E ^ { \prime }}$ was not calculated. 
${ }^{1} \mathrm{H} \mathrm{NMR}, 400 \mathrm{MHz}, \mathrm{CDCl}_{3}, 25{ }^{\circ} \mathrm{C}$

$$
M e \underbrace{\mathrm{O}}_{\substack{\mathrm{Me} \\ \mathrm{Me}}} \mathrm{Se}
$$
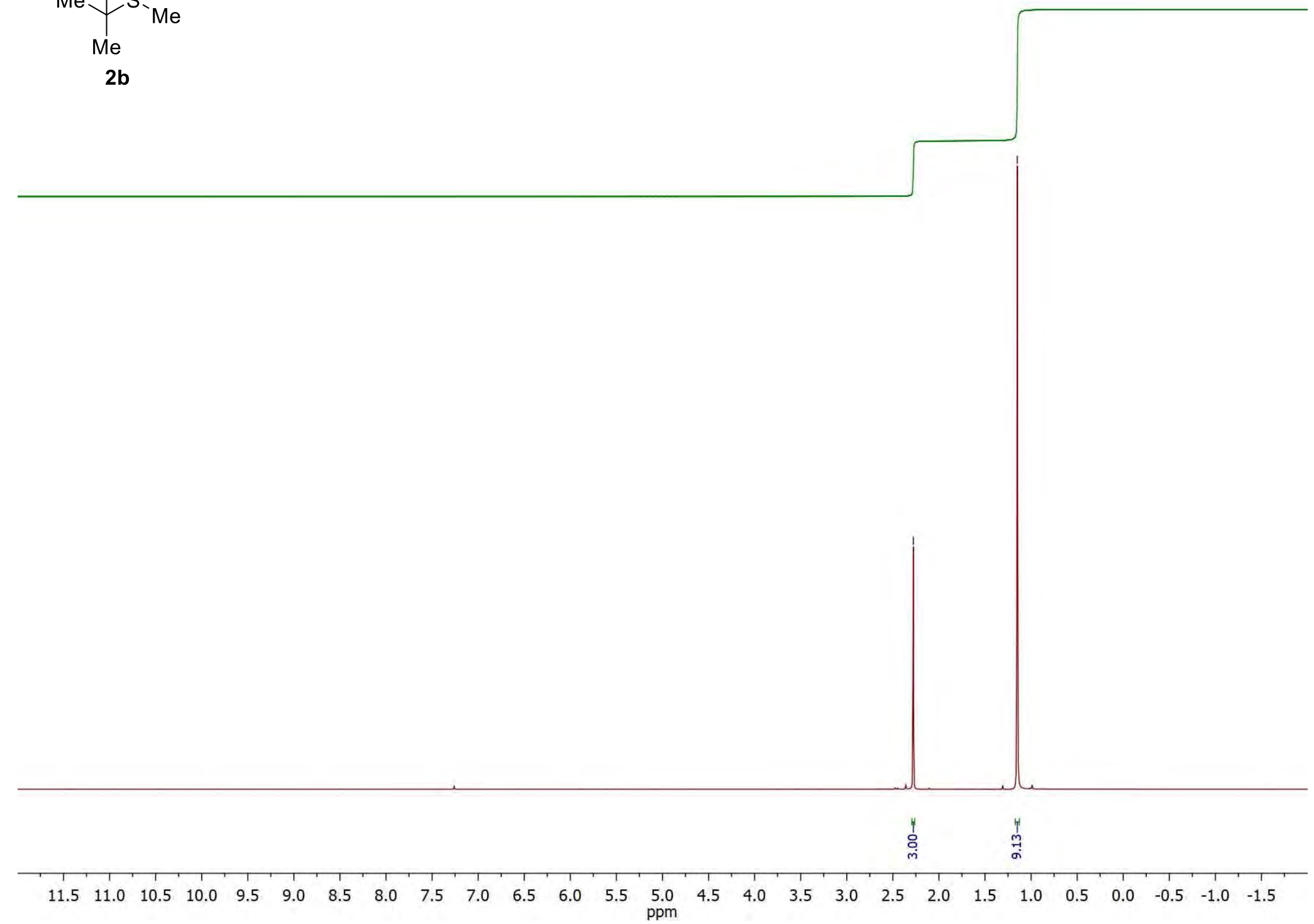
${ }^{13} \mathrm{C} \mathrm{NMR}, 100 \mathrm{MHz}, \mathrm{CDCl}_{3}, 25{ }^{\circ} \mathrm{C}$

$$
\begin{aligned}
& M e \underbrace{M e ~ I I ~}_{M e} \\
& \text { 2b }
\end{aligned}
$$

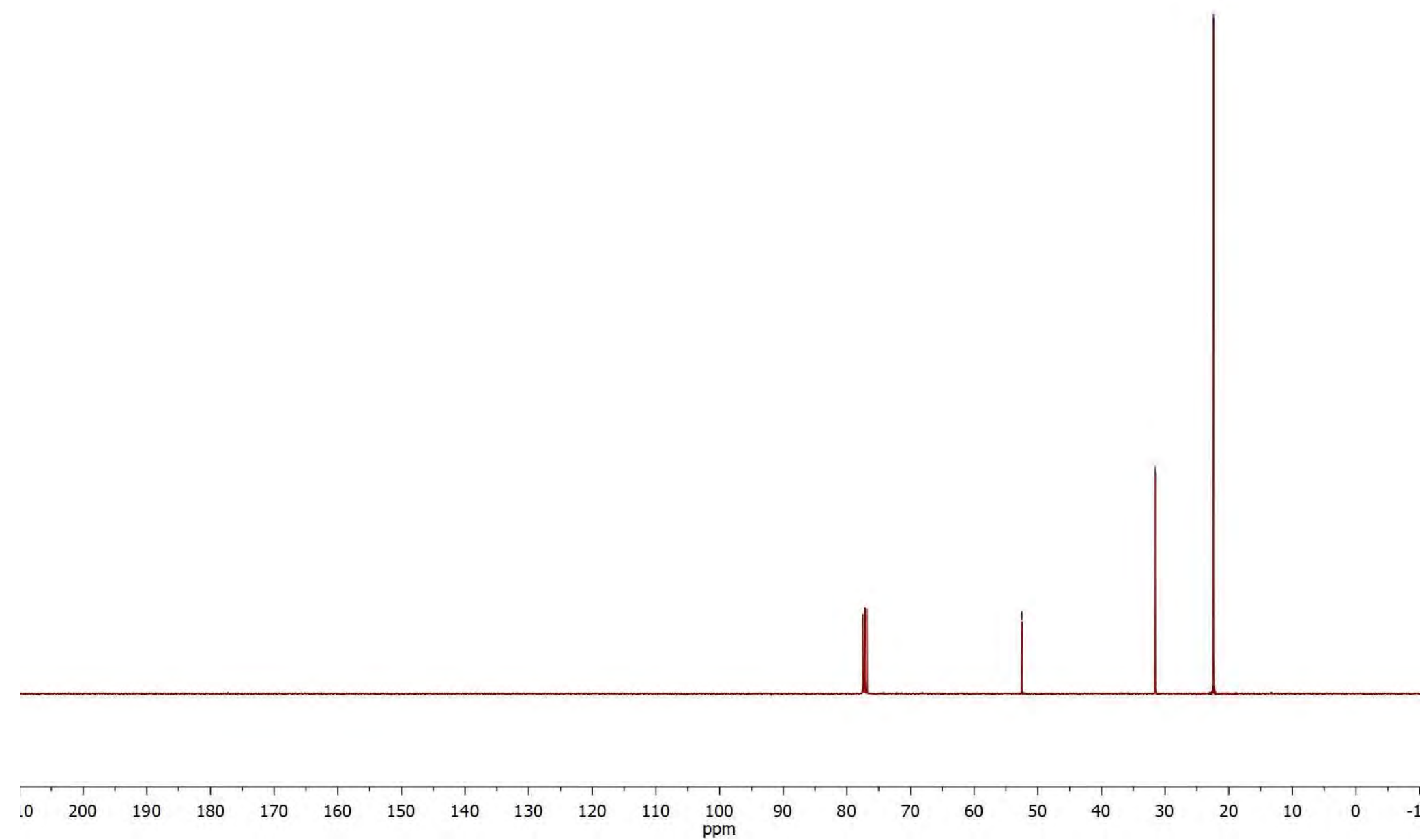


${ }^{1} \mathrm{H} \mathrm{NMR}, 400 \mathrm{MHz}, \mathrm{CDCl}_{3}, 25^{\circ} \mathrm{C}$

$$
M e \underbrace{M e ~}_{2 c} S
$$
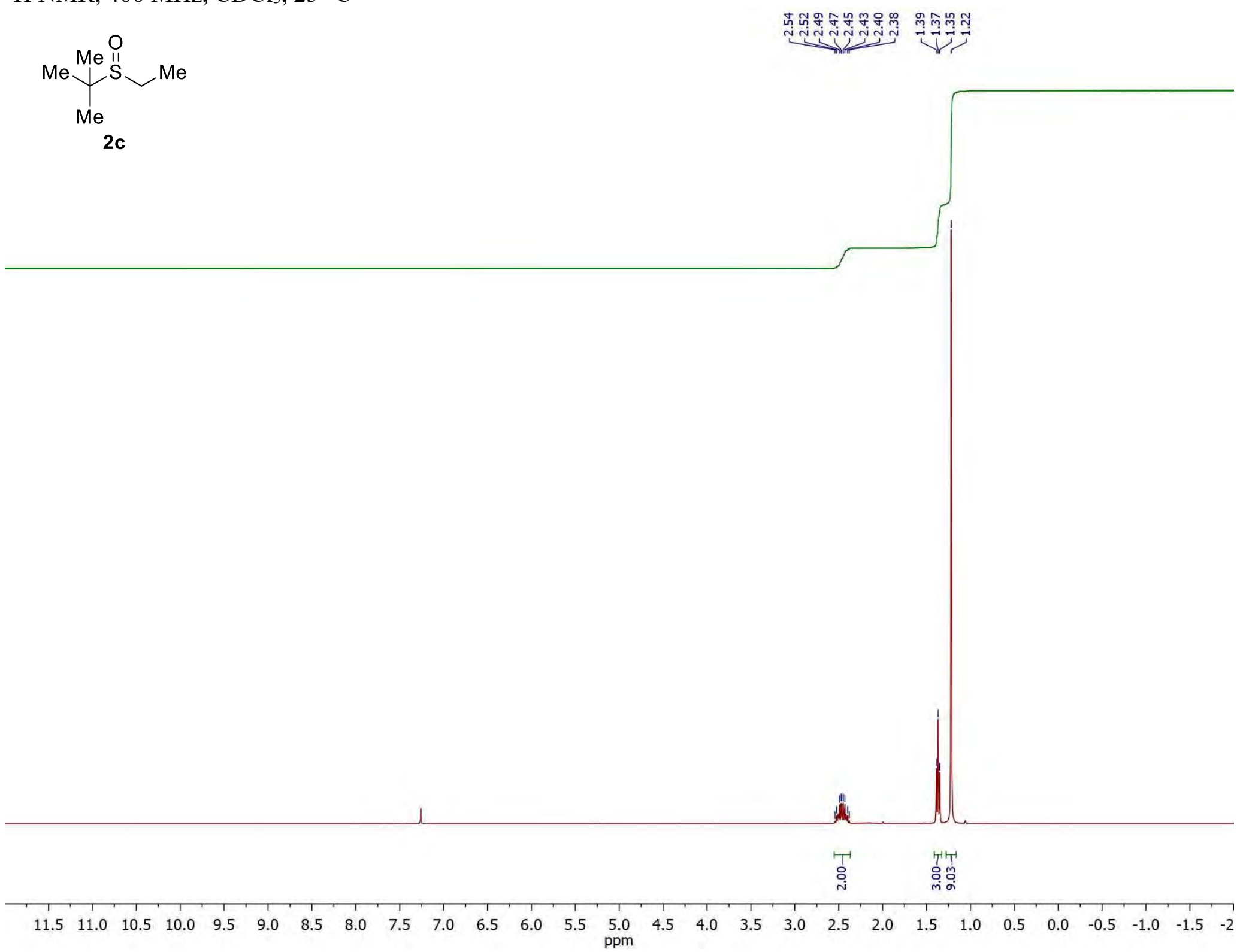
${ }^{13} \mathrm{C} \mathrm{NMR}, 100 \mathrm{MHz}, \mathrm{CDCl}_{3}, 25{ }^{\circ} \mathrm{C}$<smiles>CCS(=O)C(C)(C)C</smiles>

2c 
${ }^{1} \mathrm{H} \mathrm{NMR}, 400 \mathrm{MHz}, \mathrm{CDCl}_{3}, 25{ }^{\circ} \mathrm{C}$
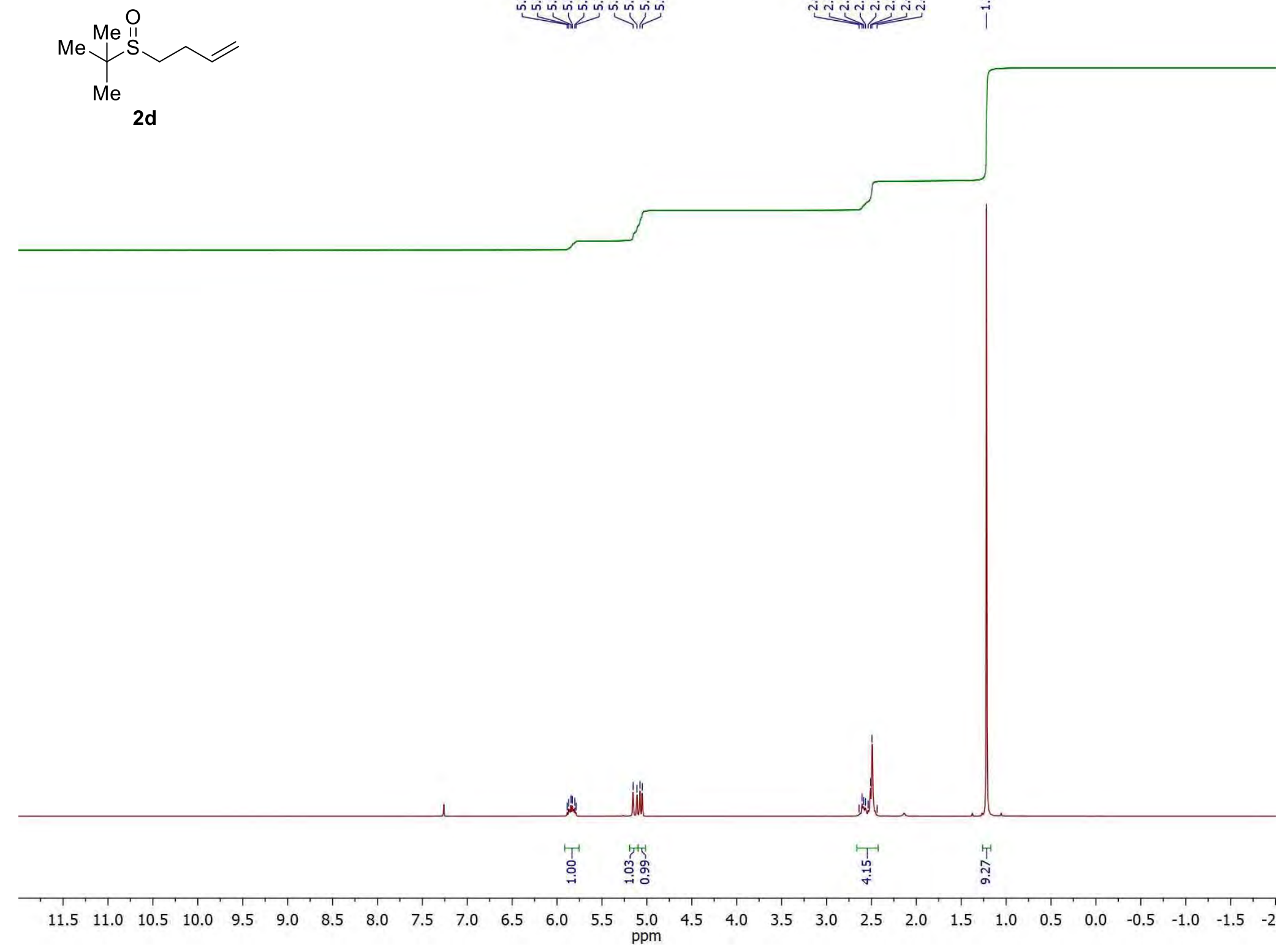
${ }^{13} \mathrm{C} \mathrm{NMR}, 100 \mathrm{MHz}, \mathrm{CDCl}_{3}, 25{ }^{\circ} \mathrm{C}$
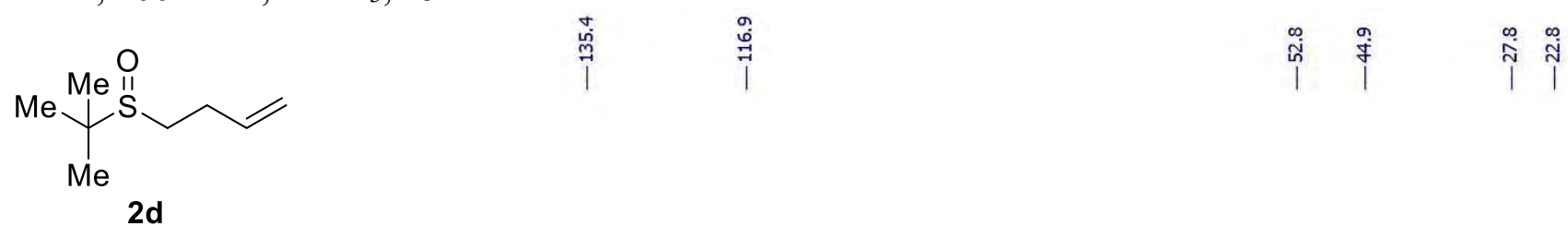

2d

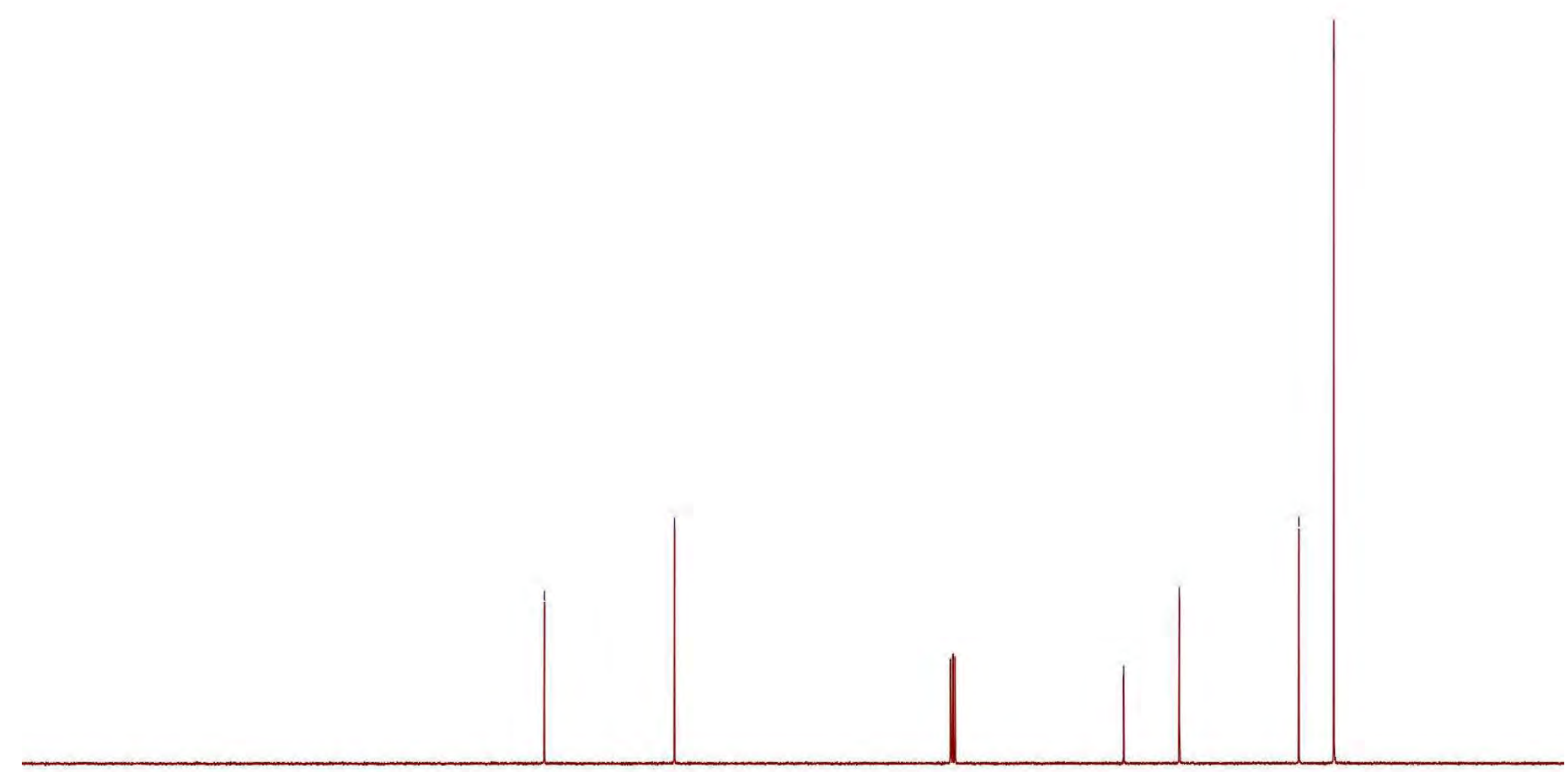

$0 \quad 200 \quad 190$ 
${ }^{1} \mathrm{H} \mathrm{NMR}, 400 \mathrm{MHz}, \mathrm{CDCl}_{3}, 25^{\circ} \mathrm{C}$

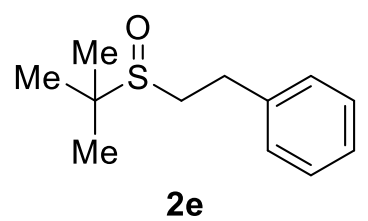

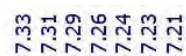

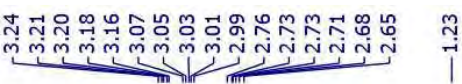
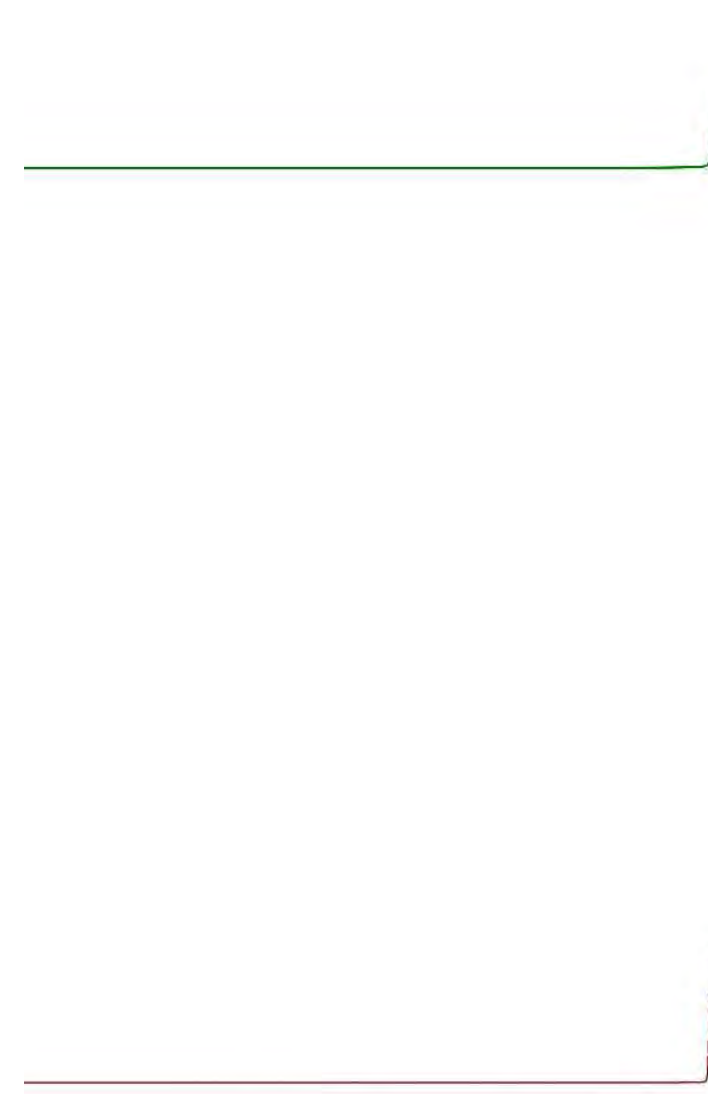

h

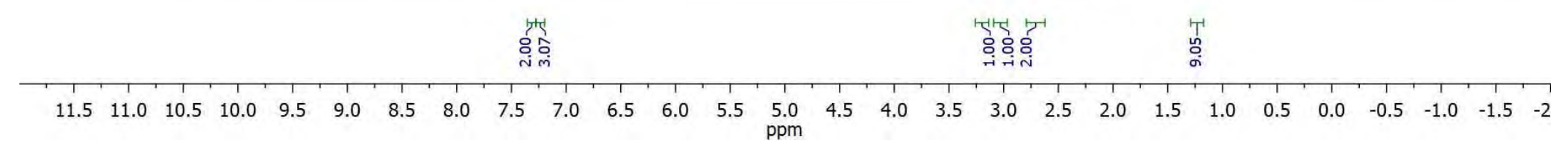


${ }^{13} \mathrm{C} \mathrm{NMR}, 100 \mathrm{MHz}, \mathrm{CDCl}_{3}, 25{ }^{\circ} \mathrm{C}$
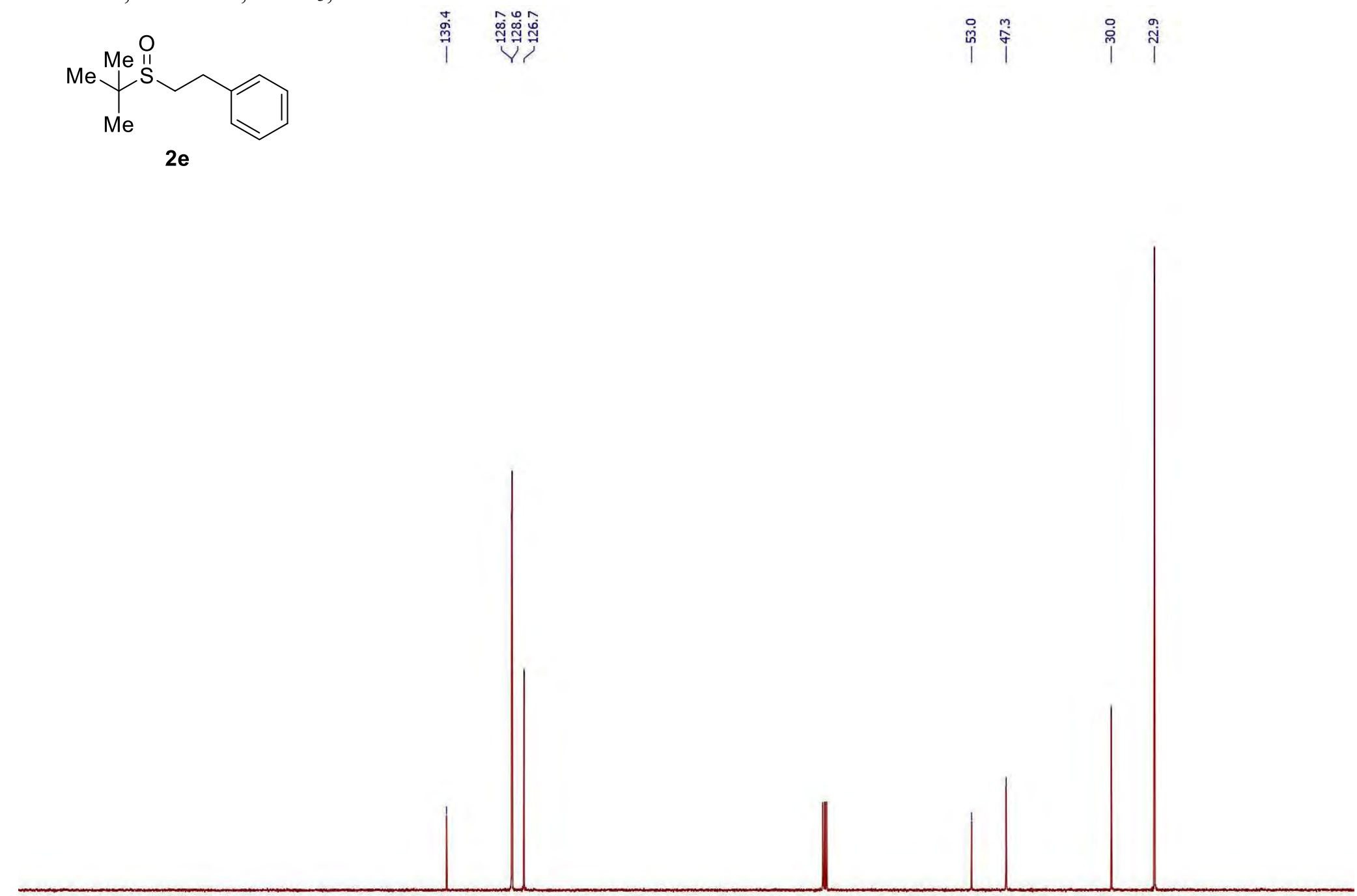

$10 \quad 200 \quad 190$ 
${ }^{1} \mathrm{H} \mathrm{NMR}, 400 \mathrm{MHz}, \mathrm{CDCl}_{3}, 25^{\circ} \mathrm{C}$

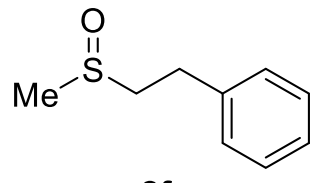

$2 f$

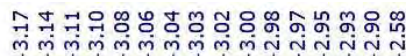

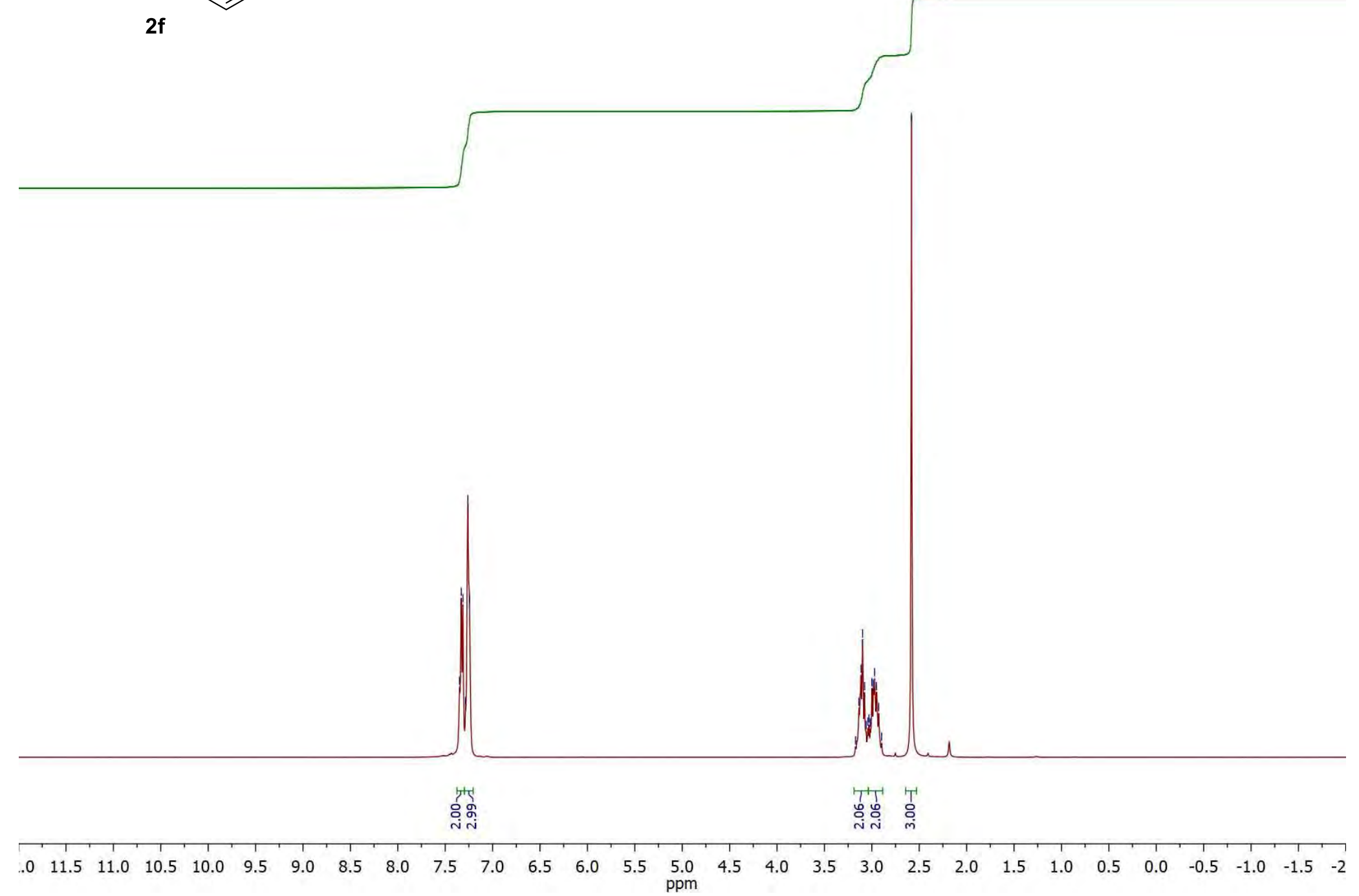


${ }^{13} \mathrm{C}$ NMR, $100 \mathrm{MHz}, \mathrm{CDCl}_{3}, 25{ }^{\circ} \mathrm{C}$<smiles>CCCS(C)=O</smiles>

$2 f$

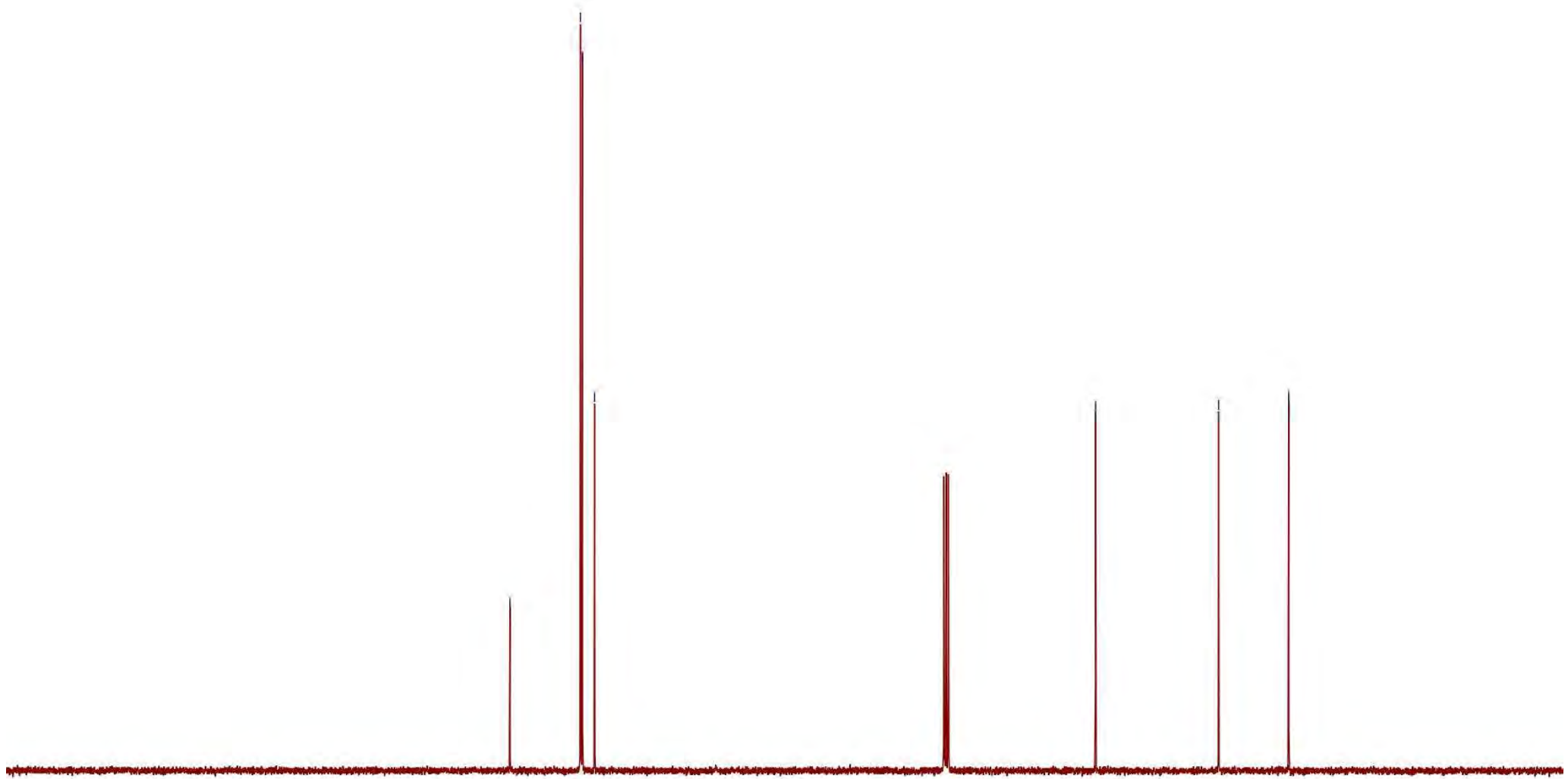

$0 \quad 200 \quad 190$ 
${ }^{1} \mathrm{H} \mathrm{NMR}, 400 \mathrm{MHz}, \mathrm{CDCl}_{3}, 25{ }^{\circ} \mathrm{C}$

$\mathrm{MeO}$

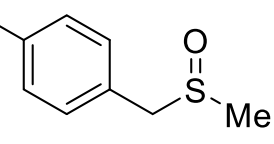

$2 \mathrm{~g}$

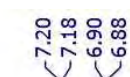

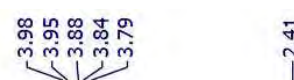

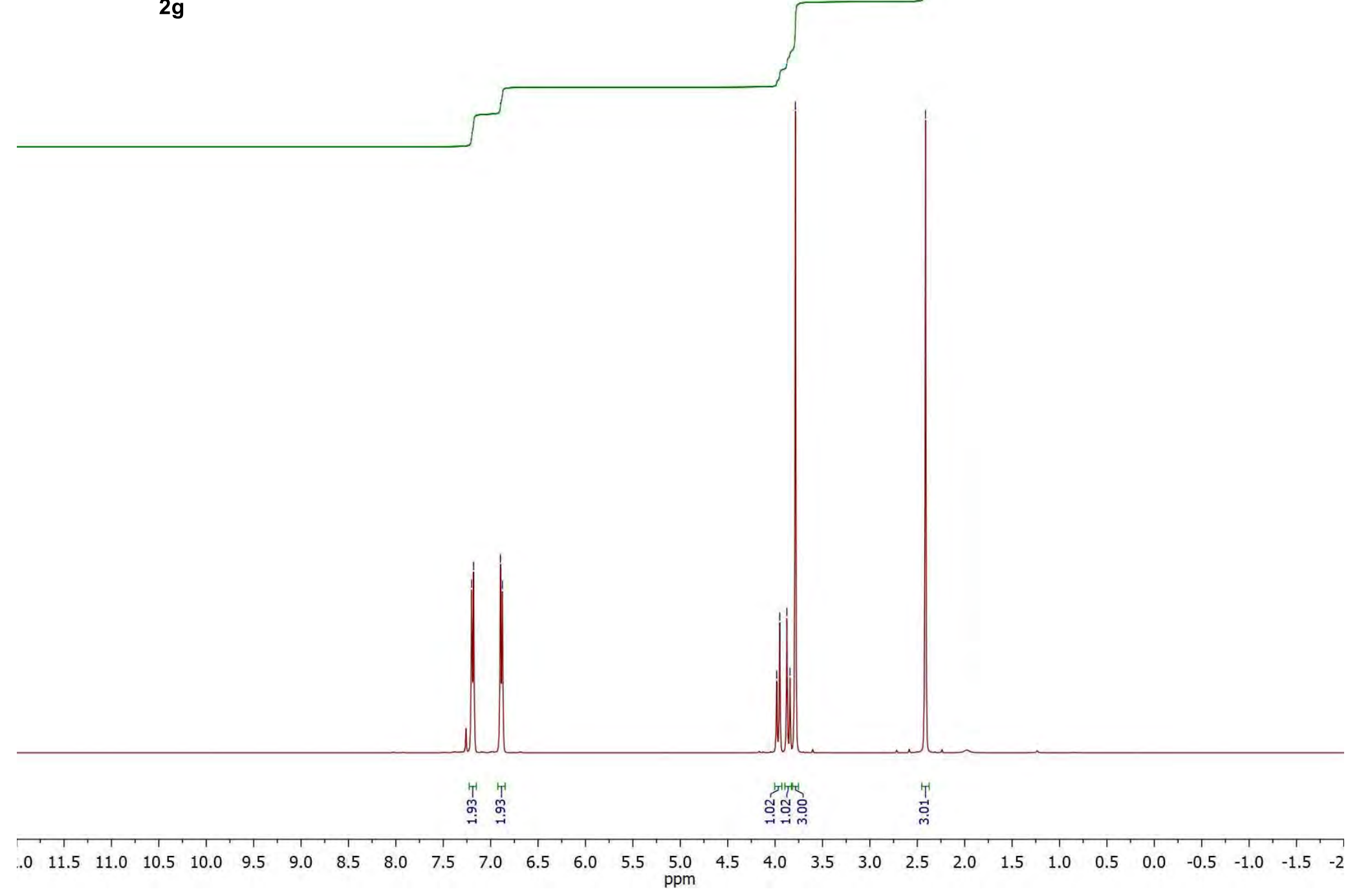


Chemoselective $\alpha$-Sulfidation of Amides Using Sulfoxide Reagents

M. Leypold, K. A. D’Angelo and M. Movassaghi

Page S114 / S183

${ }^{13} \mathrm{C} \mathrm{NMR}, 100 \mathrm{MHz}, \mathrm{CDCl}_{3}, 25^{\circ} \mathrm{C}$<smiles>COc1ccc(CS(C)=O)cc1</smiles>

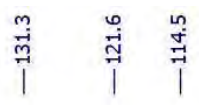

今ે

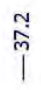

2g
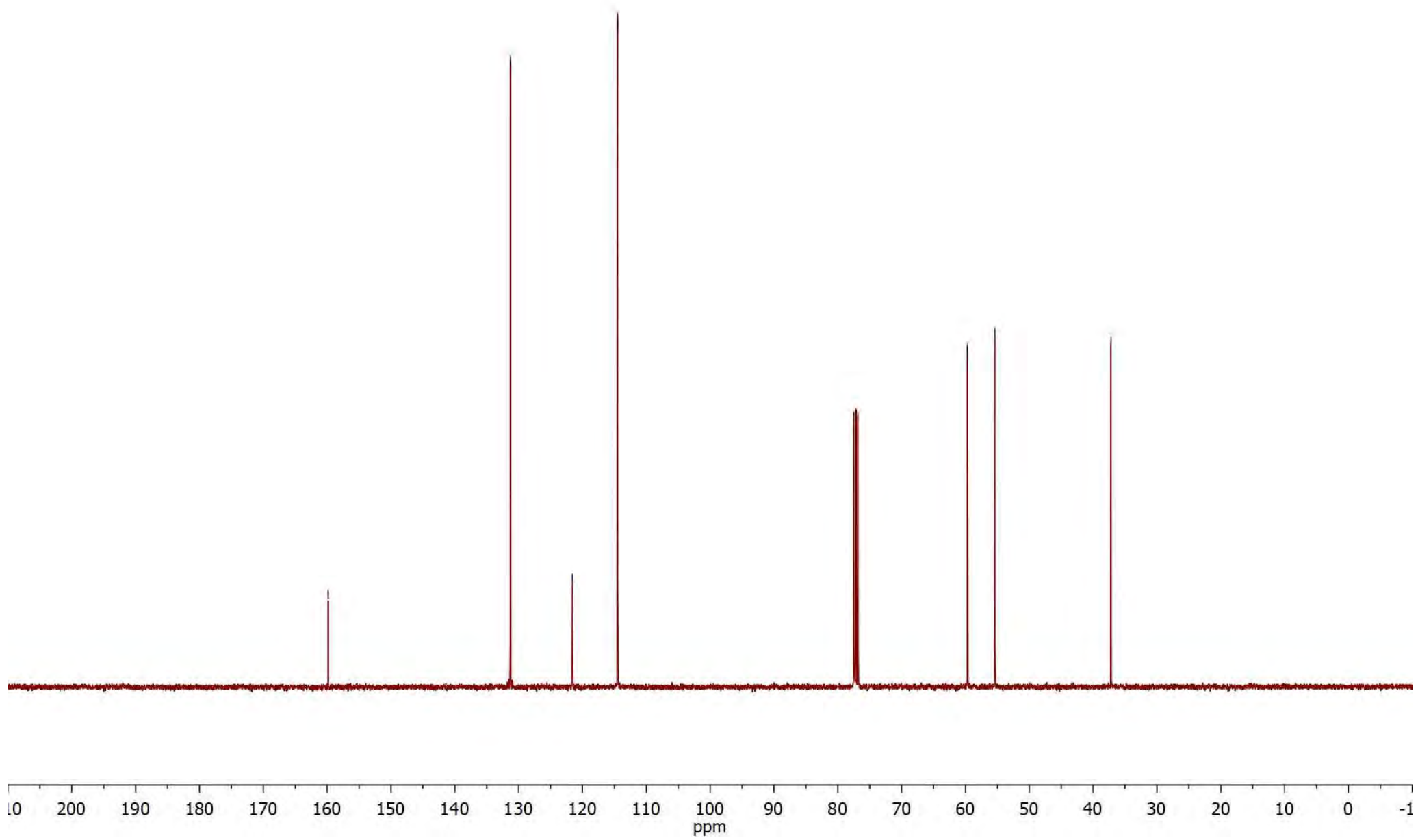
${ }^{1} \mathrm{H}$ NMR, $400 \mathrm{MHz}, \mathrm{CD}_{2} \mathrm{Cl}_{2}, 25^{\circ} \mathrm{C}$

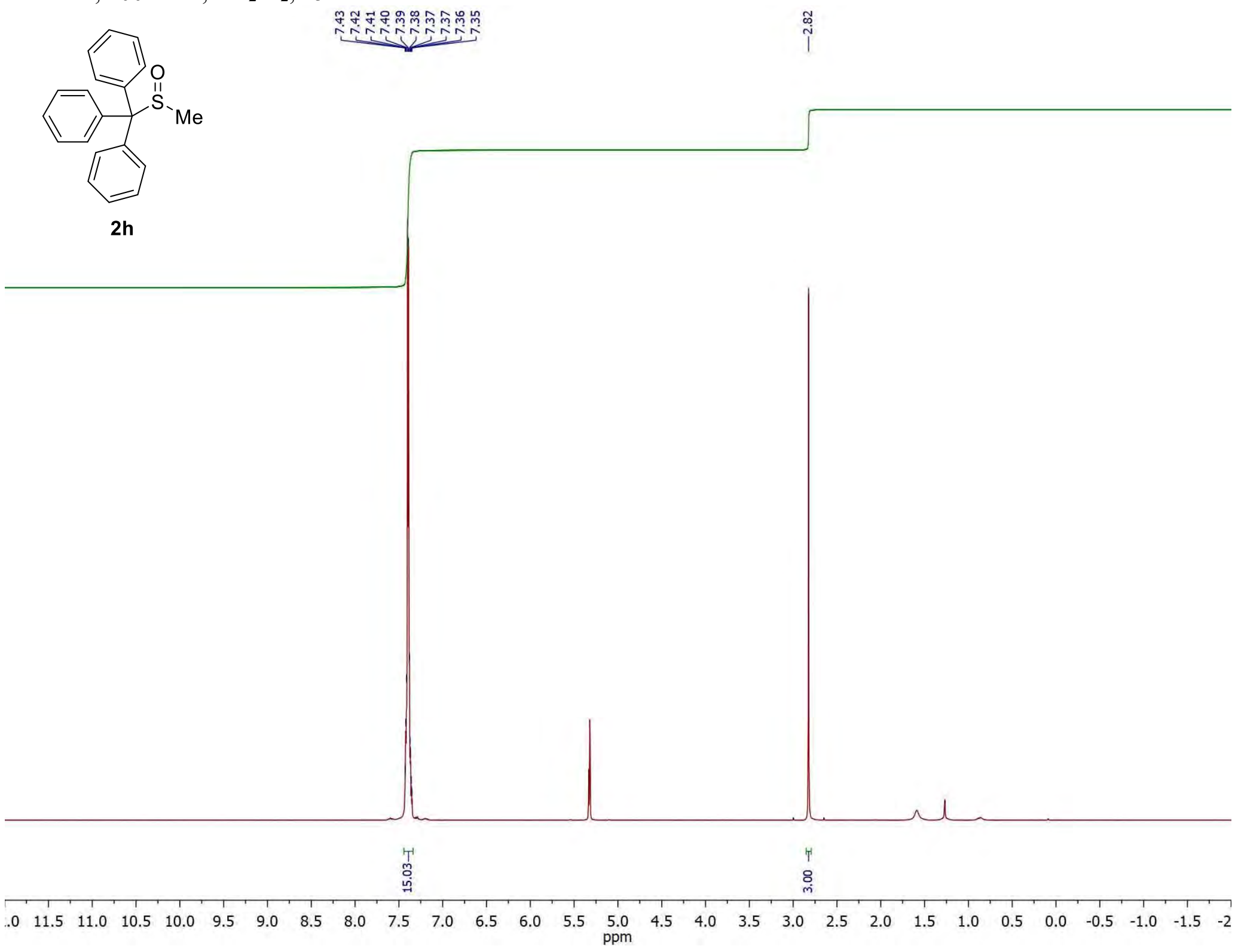


${ }^{13} \mathrm{C}$ NMR, $100 \mathrm{MHz}, \mathrm{CD}_{2} \mathrm{Cl}_{2}, 25{ }^{\circ} \mathrm{C}$

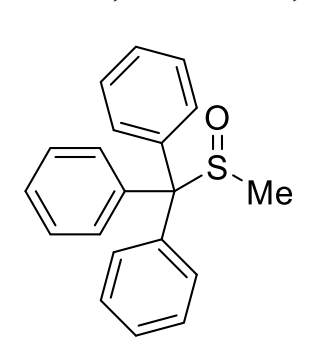

$2 \mathrm{~h}$

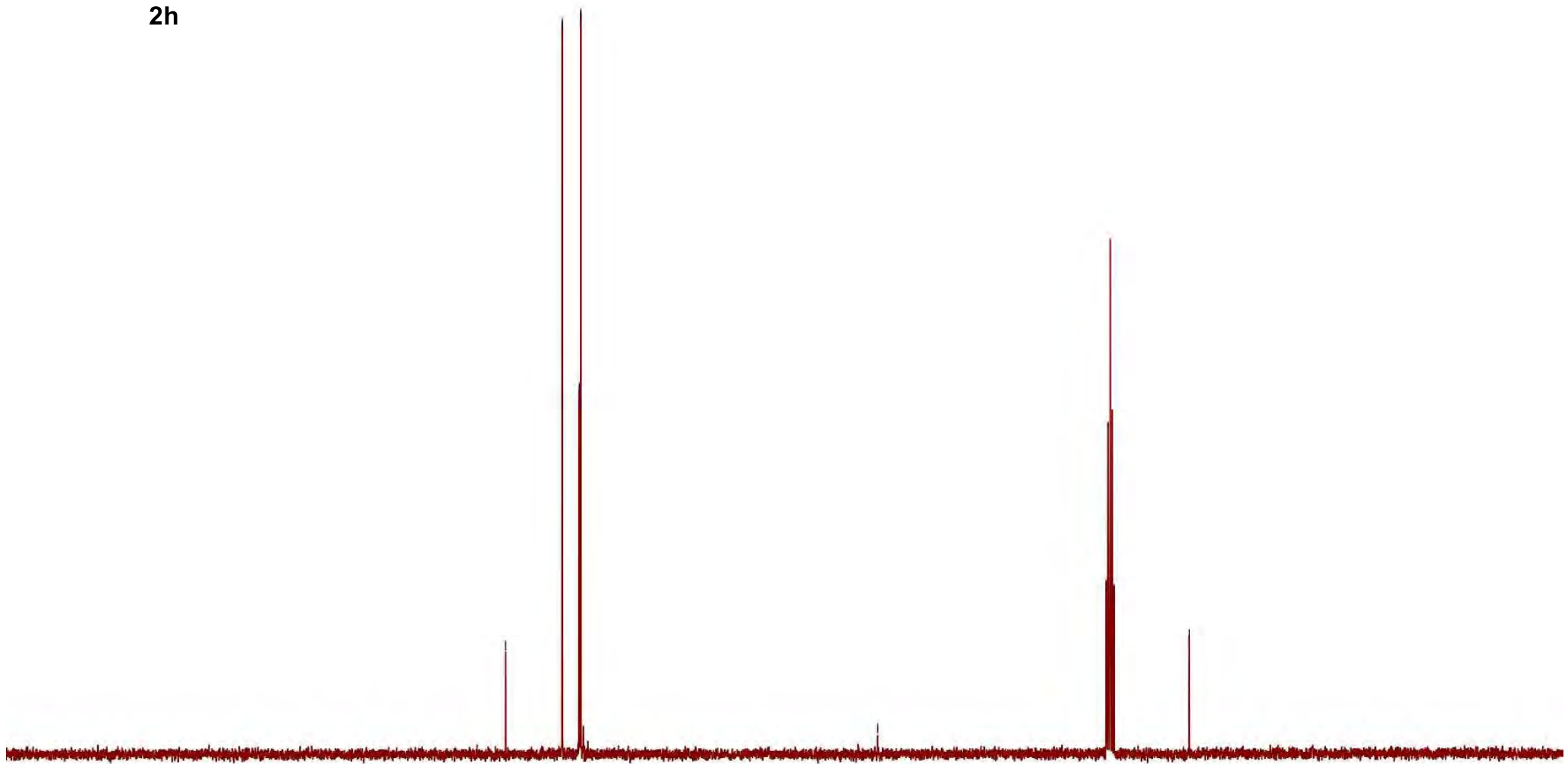

$10 \quad 200 \quad 190$ 
${ }^{1} \mathrm{H} \mathrm{NMR}, 400 \mathrm{MHz}, \mathrm{CDCl}_{3}, 25^{\circ} \mathrm{C}$
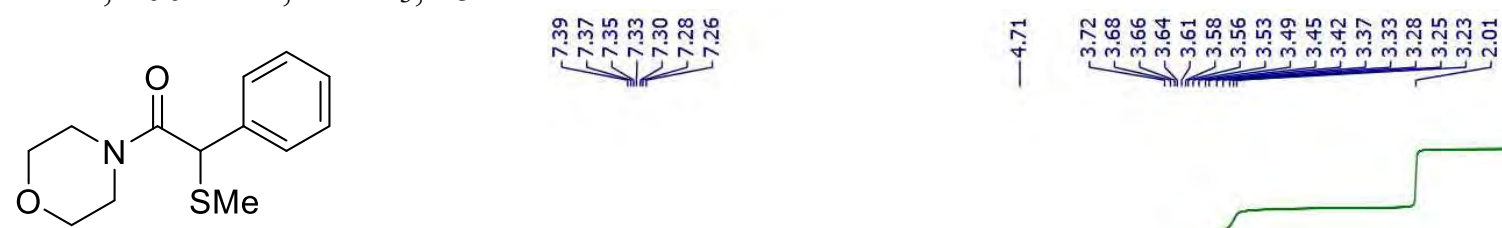

$4 a$

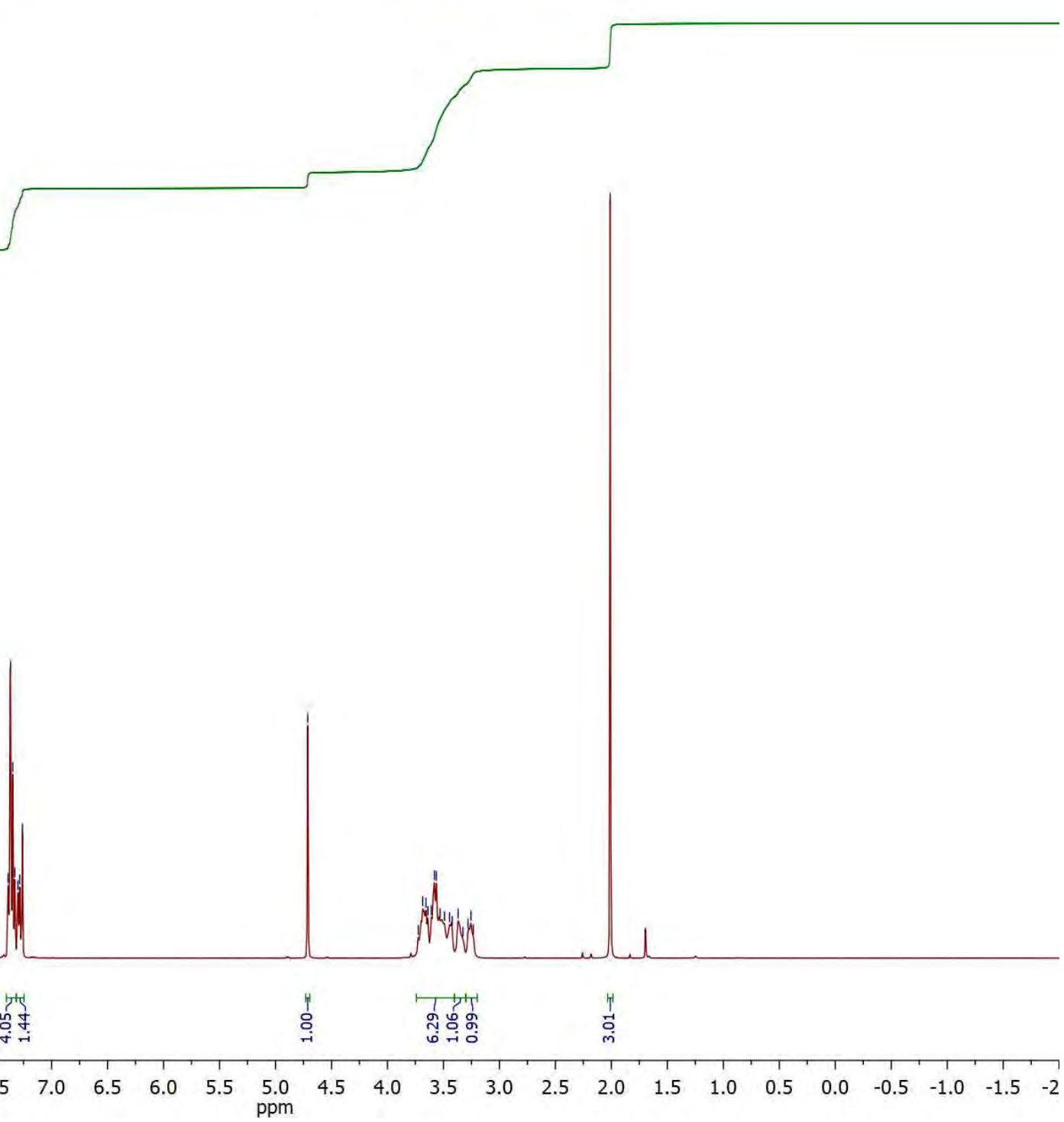


${ }^{13} \mathrm{C} \mathrm{NMR}, 100 \mathrm{MHz}, \mathrm{CDCl}_{3}, 25{ }^{\circ} \mathrm{C}$

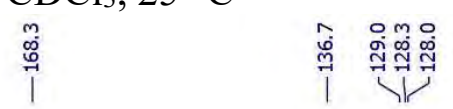

空

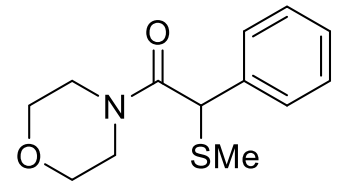

$4 a$

$0 \quad 200 \quad 190$ 
${ }^{1} \mathrm{H} \mathrm{NMR}, 500 \mathrm{MHz}, \mathrm{CDCl}_{3}, 30{ }^{\circ} \mathrm{C}$
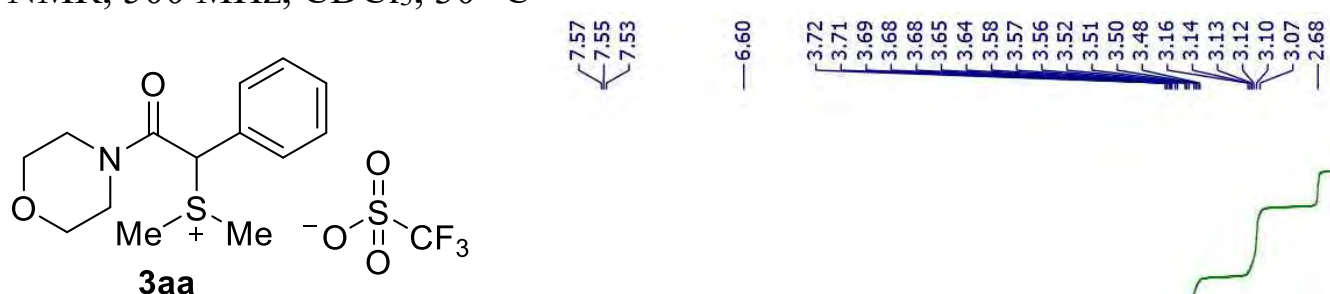

3aa
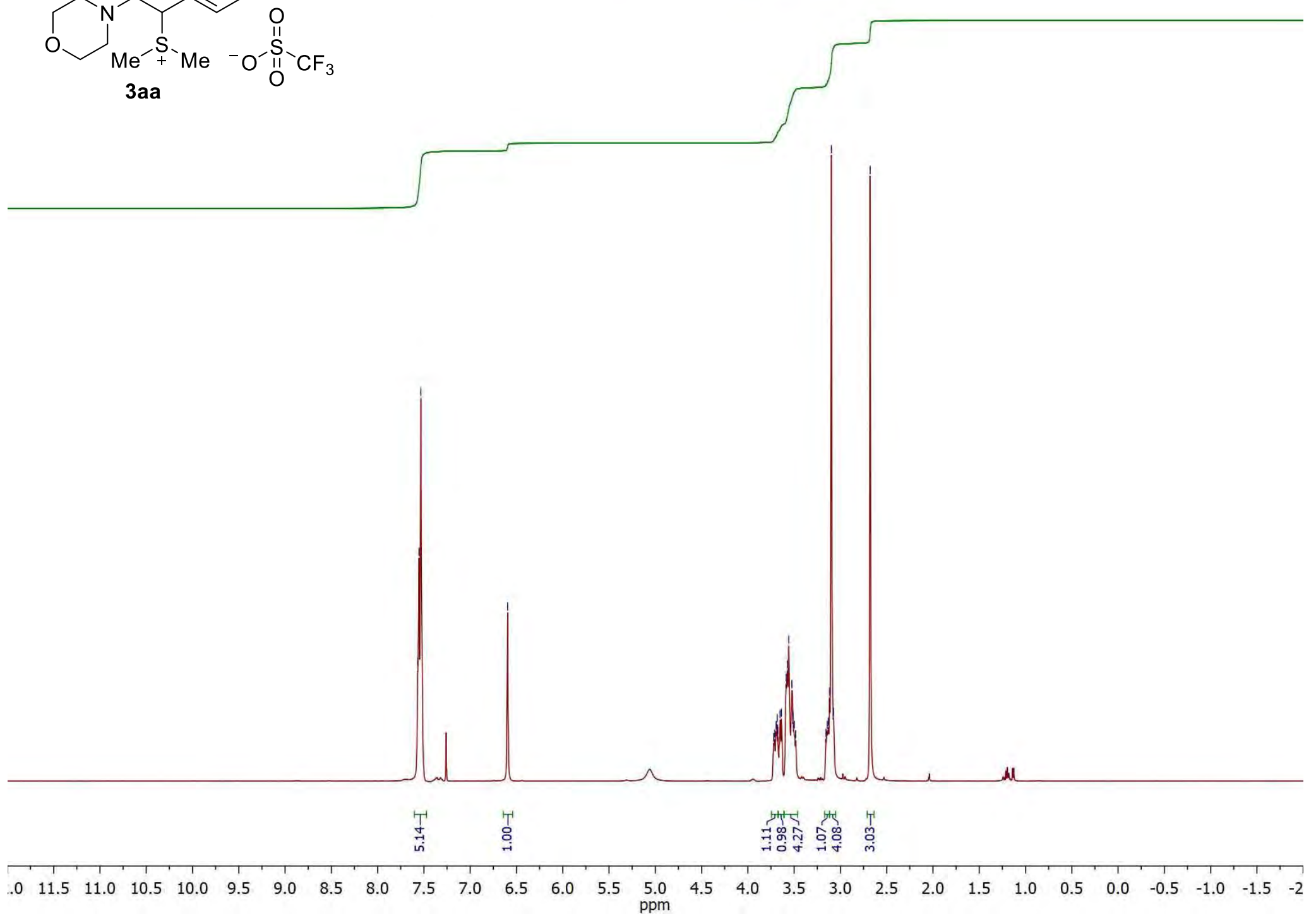
${ }^{13} \mathrm{C} \mathrm{NMR}, 125 \mathrm{MHz}, \mathrm{CDCl}_{3}, 30{ }^{\circ} \mathrm{C}$
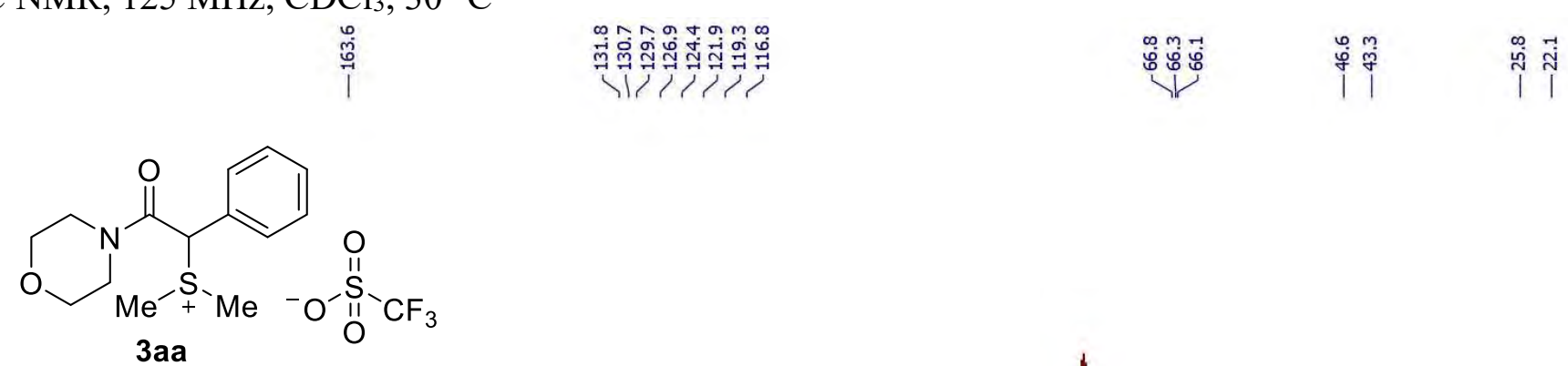

3aa

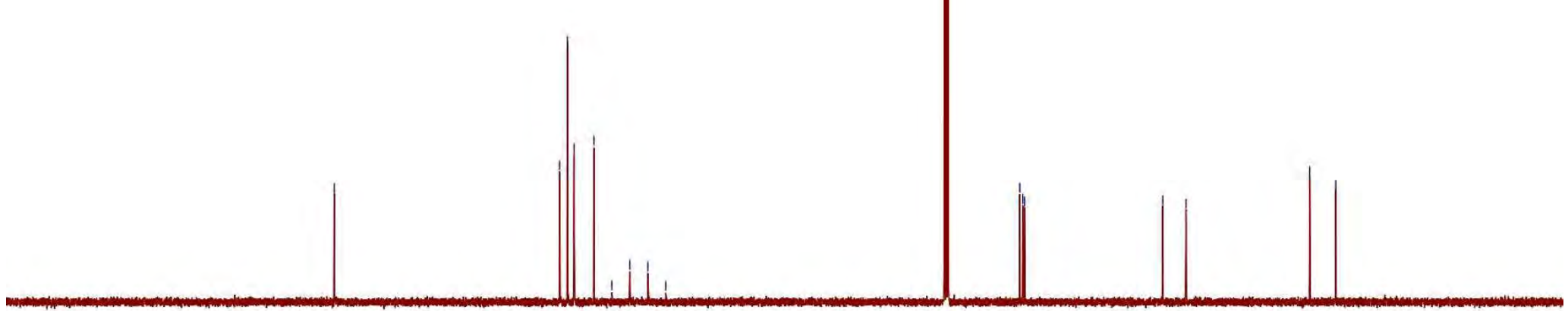

$\begin{array}{llll}0 & 200 & 190 & 180\end{array}$ 
${ }^{19} \mathrm{~F} \mathrm{NMR}, 470 \mathrm{MHz}, \mathrm{CDCl}_{3}, 30{ }^{\circ} \mathrm{C}$

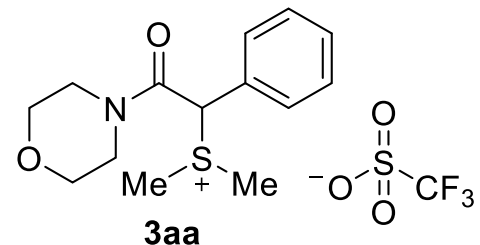

3aa

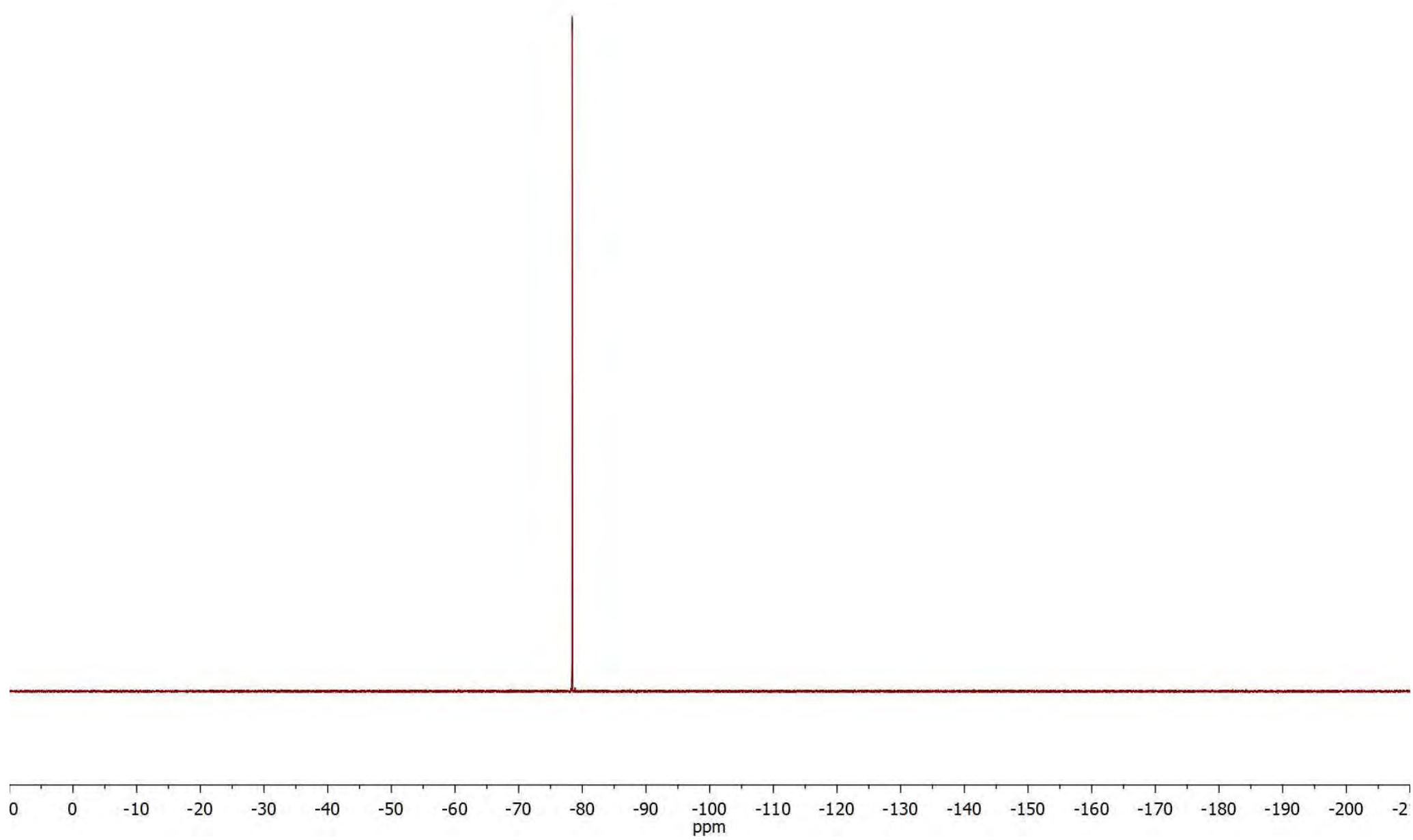


${ }^{1} \mathrm{H} \mathrm{NMR}, 400 \mathrm{MHz}, \mathrm{CDCl}_{3}, 25^{\circ} \mathrm{C}$

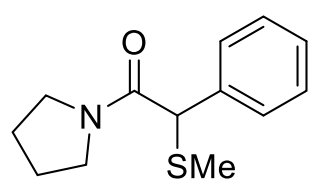

\section{ำ}

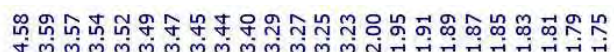

ن

$4 b$

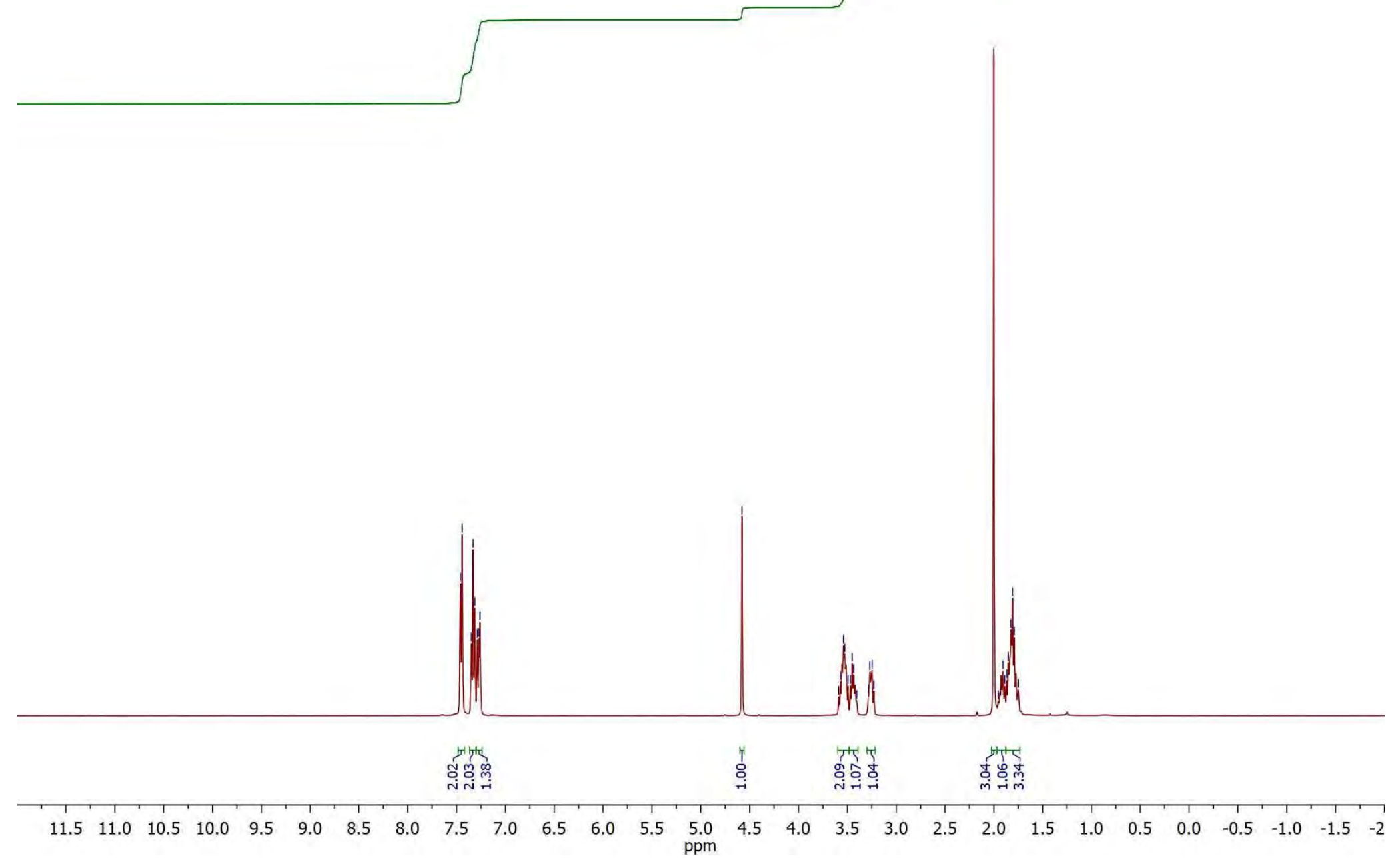


${ }^{13} \mathrm{C} \mathrm{NMR}, 100 \mathrm{MHz}, \mathrm{CDCl}_{3}, 25{ }^{\circ} \mathrm{C}$
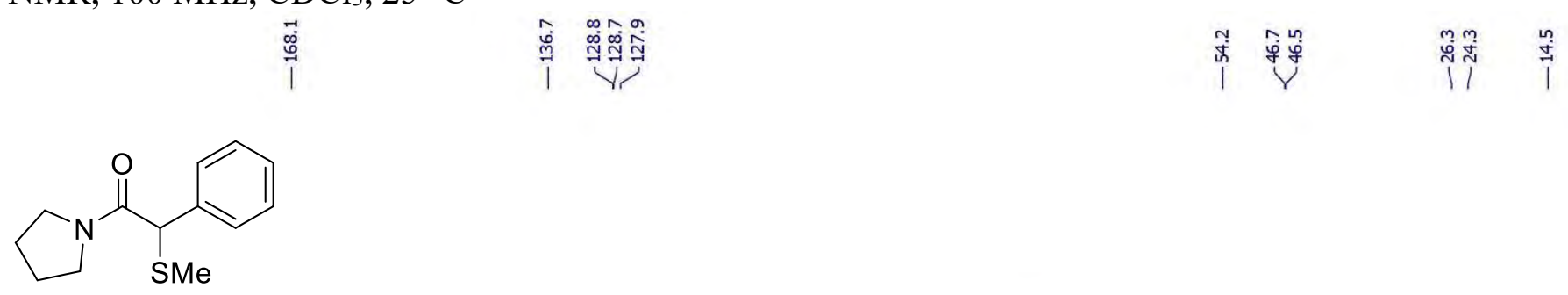

4b

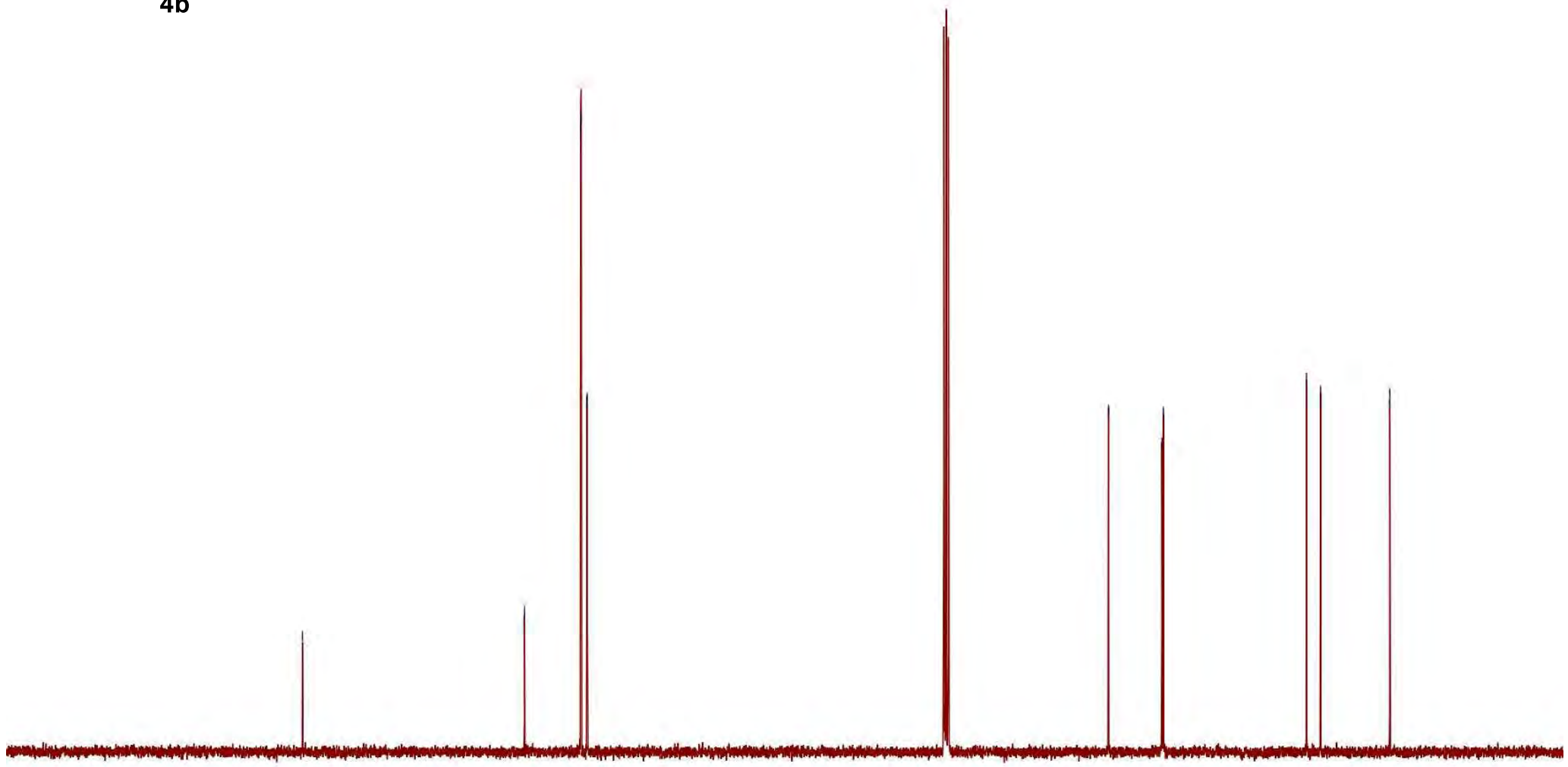

$.0 \quad 200 \quad 190$ 
${ }^{1} \mathrm{H} \mathrm{NMR}, 400 \mathrm{MHz}, \mathrm{CDCl}_{3}, 25^{\circ} \mathrm{C}$

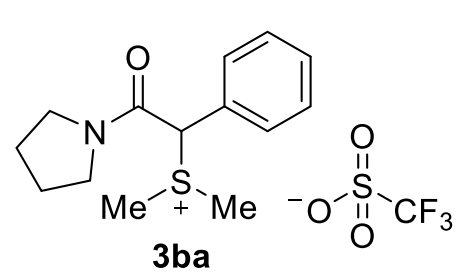

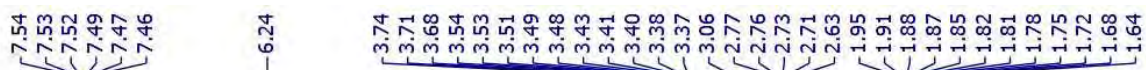
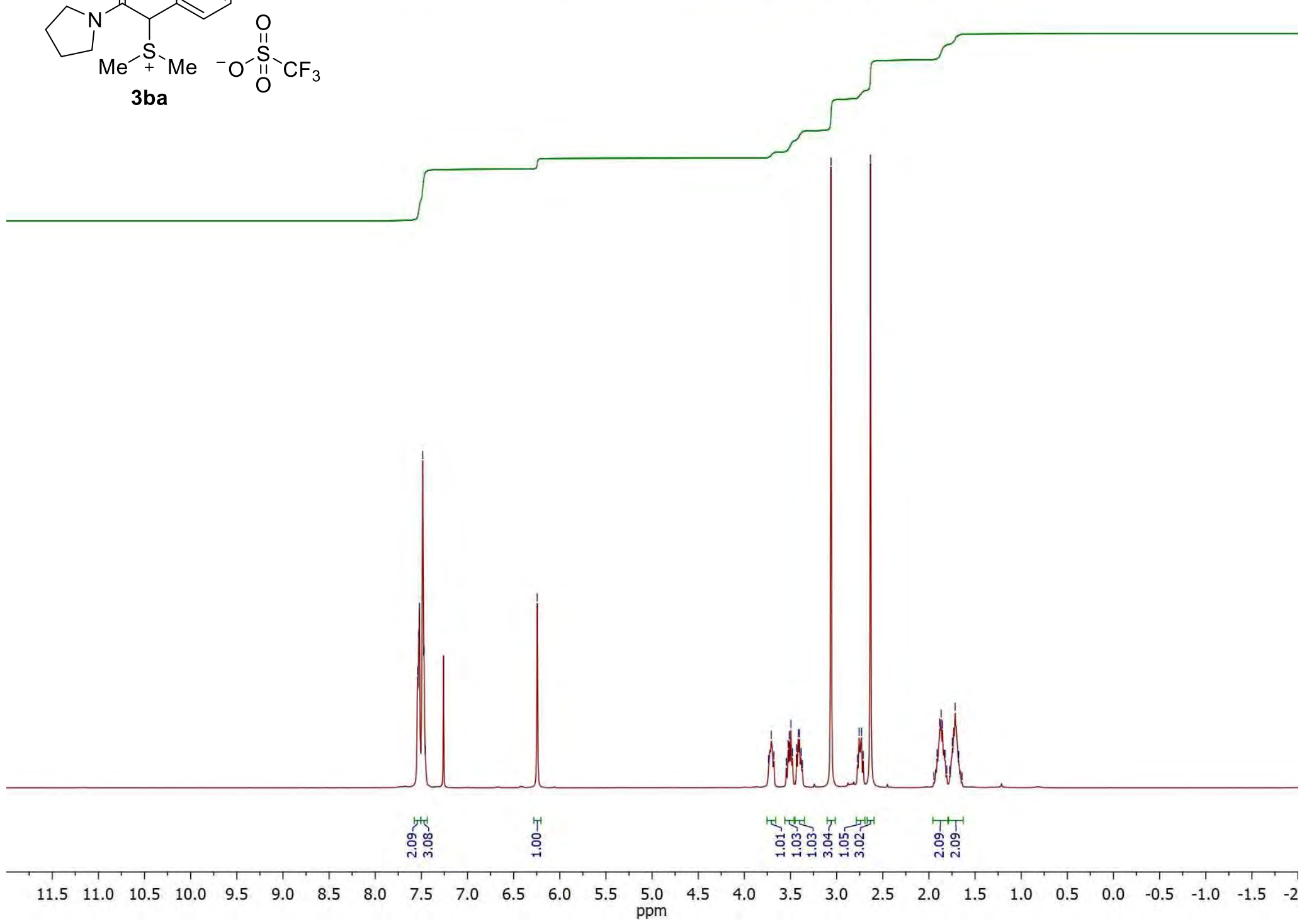
${ }^{13} \mathrm{C} \mathrm{NMR,} 100 \mathrm{MHz}, \mathrm{CDCl}_{3}, 25{ }^{\circ} \mathrm{C}$
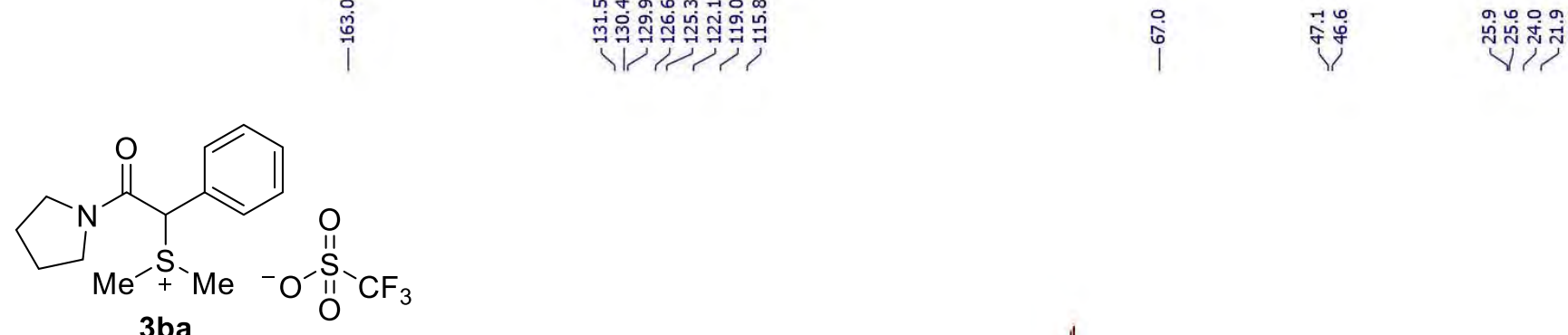

3 ba

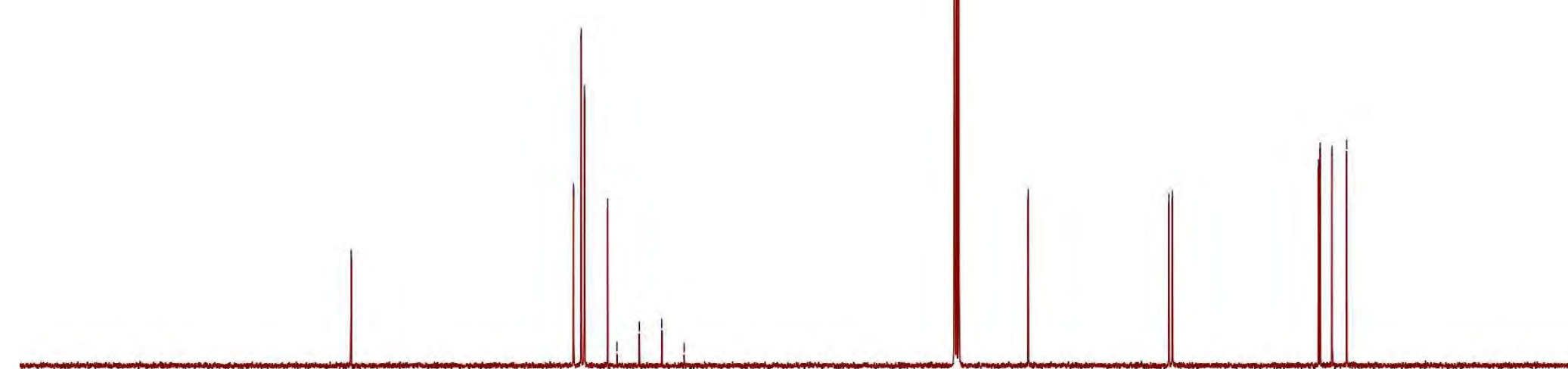

$200 \quad 190$ 
${ }^{19} \mathrm{~F} \mathrm{NMR,} 376 \mathrm{MHz}, \mathrm{CDCl}_{3}, 25{ }^{\circ} \mathrm{C}$

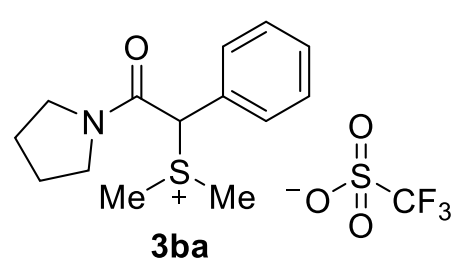

3 ba

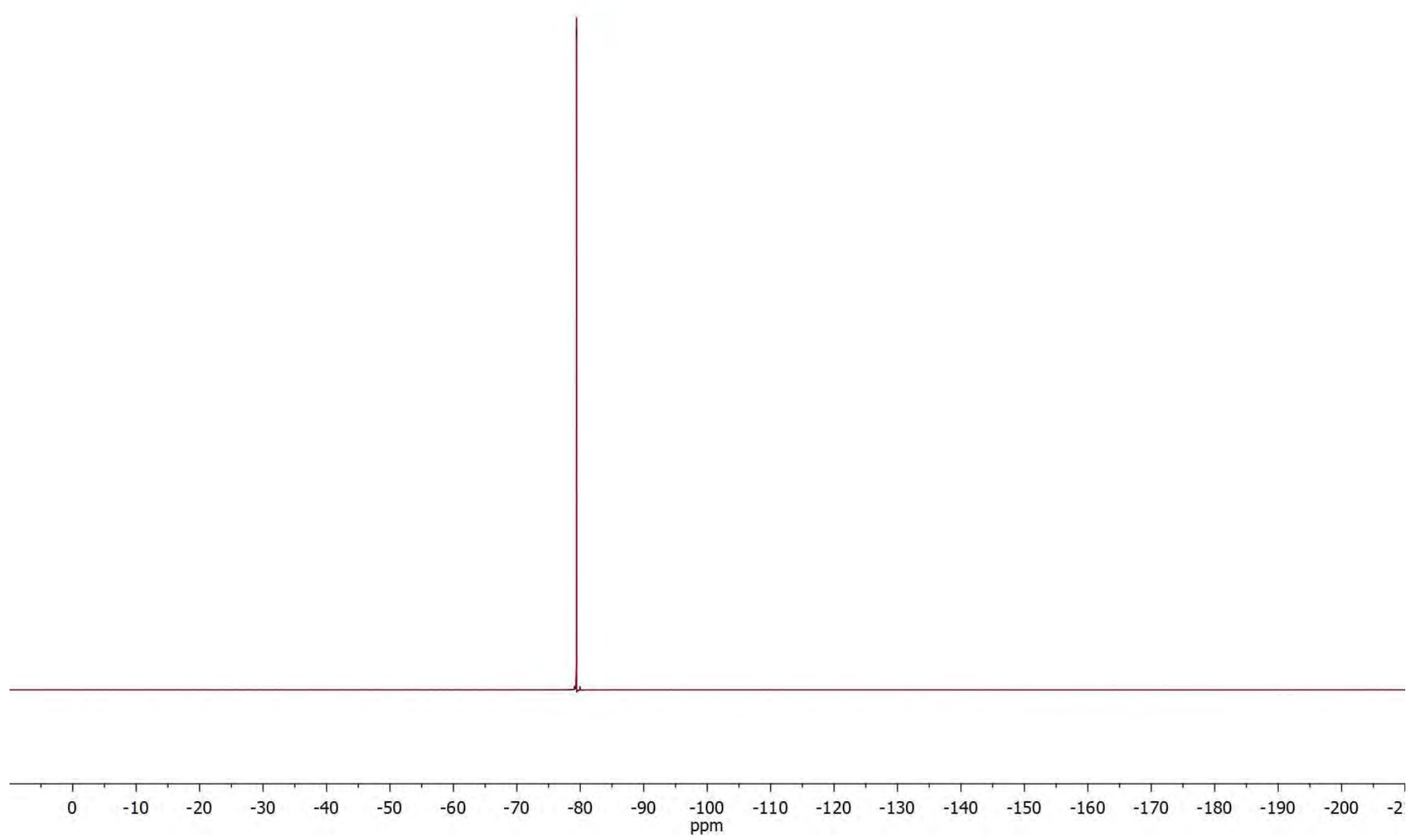


${ }^{1} \mathrm{H} \mathrm{NMR}, 400 \mathrm{MHz}, \mathrm{CDCl}_{3}, 25^{\circ} \mathrm{C}$
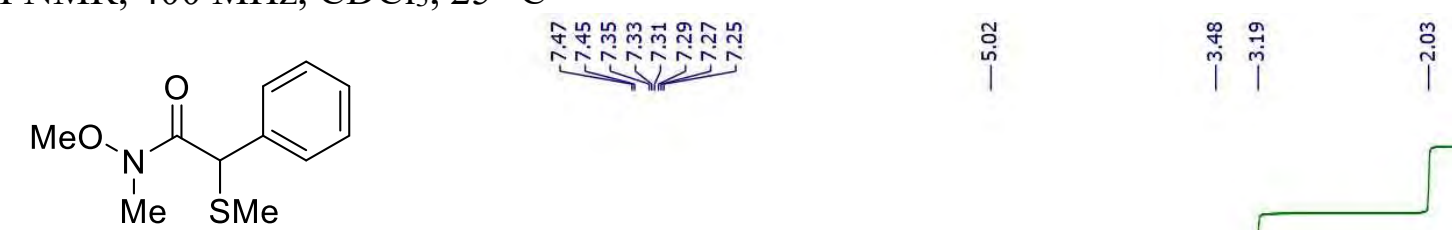

4c
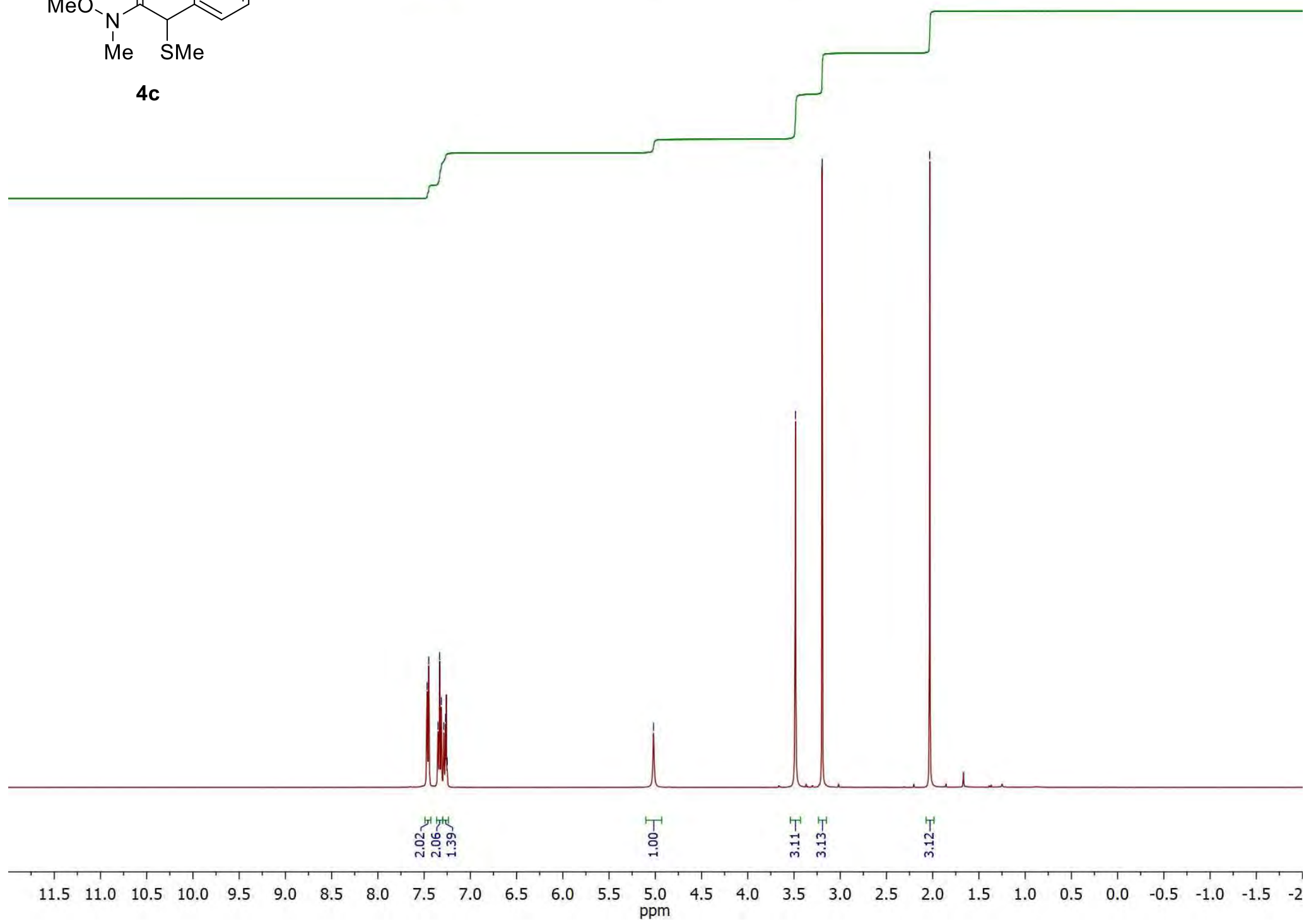
${ }^{13} \mathrm{C} \mathrm{NMR}, 100 \mathrm{MHz}, \mathrm{CDCl}_{3}, 25{ }^{\circ} \mathrm{C}$

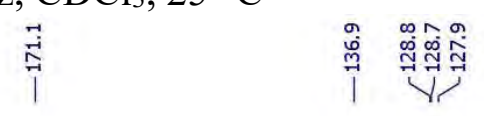

足

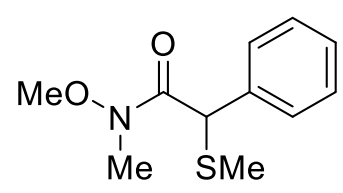

$4 c$

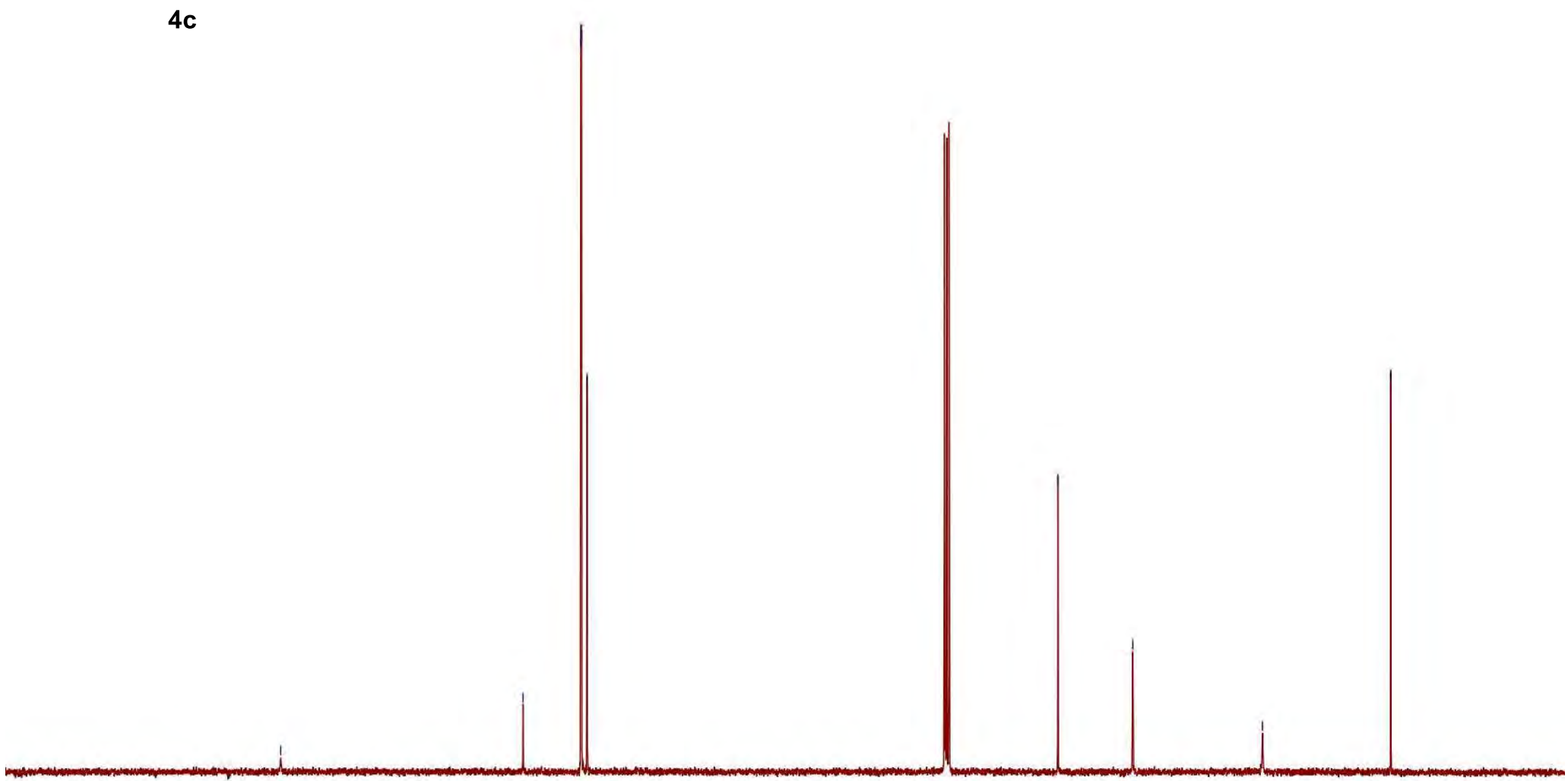

$0 \quad 200 \quad 190$ 
${ }^{1} \mathrm{H} \mathrm{NMR}, 400 \mathrm{MHz}, \mathrm{CDCl}_{3}, 25^{\circ} \mathrm{C}$

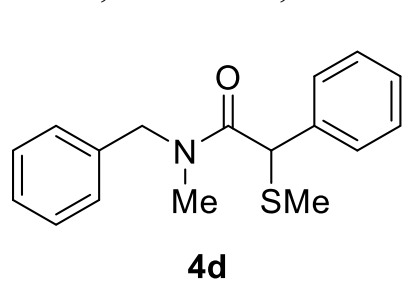

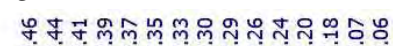

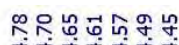

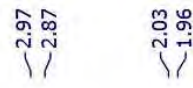

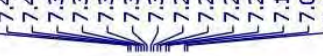

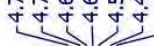

$\sqrt{2}$
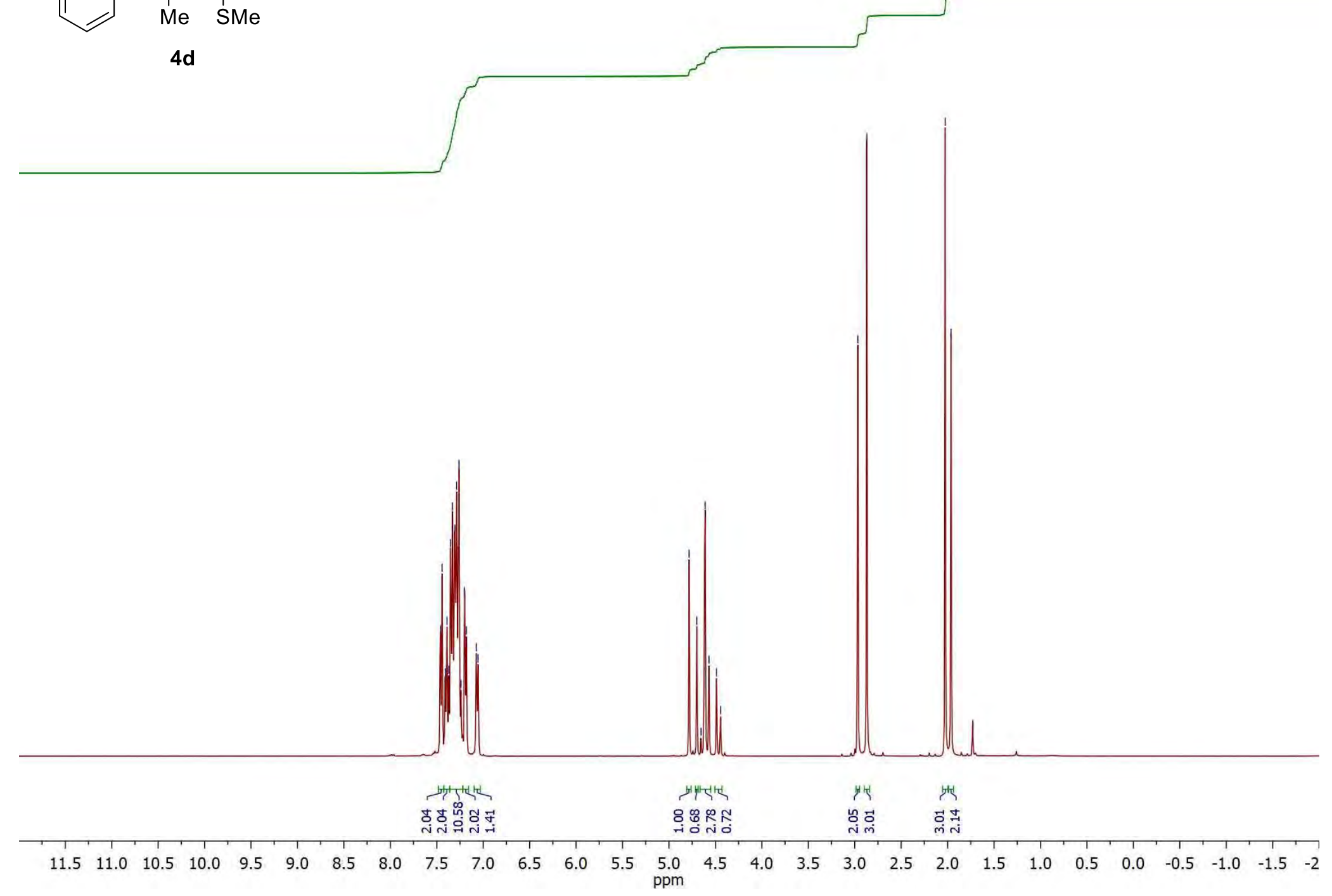
${ }^{13} \mathrm{C} \mathrm{NMR}, 100 \mathrm{MHz}, \mathrm{CDCl}_{3}, 25{ }^{\circ} \mathrm{C}$

עึ 说:

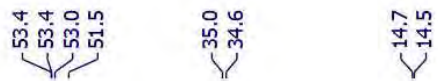

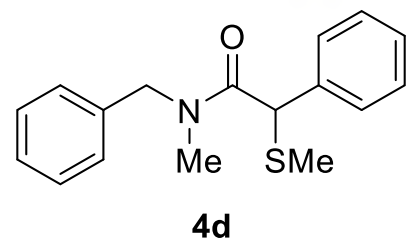

4d

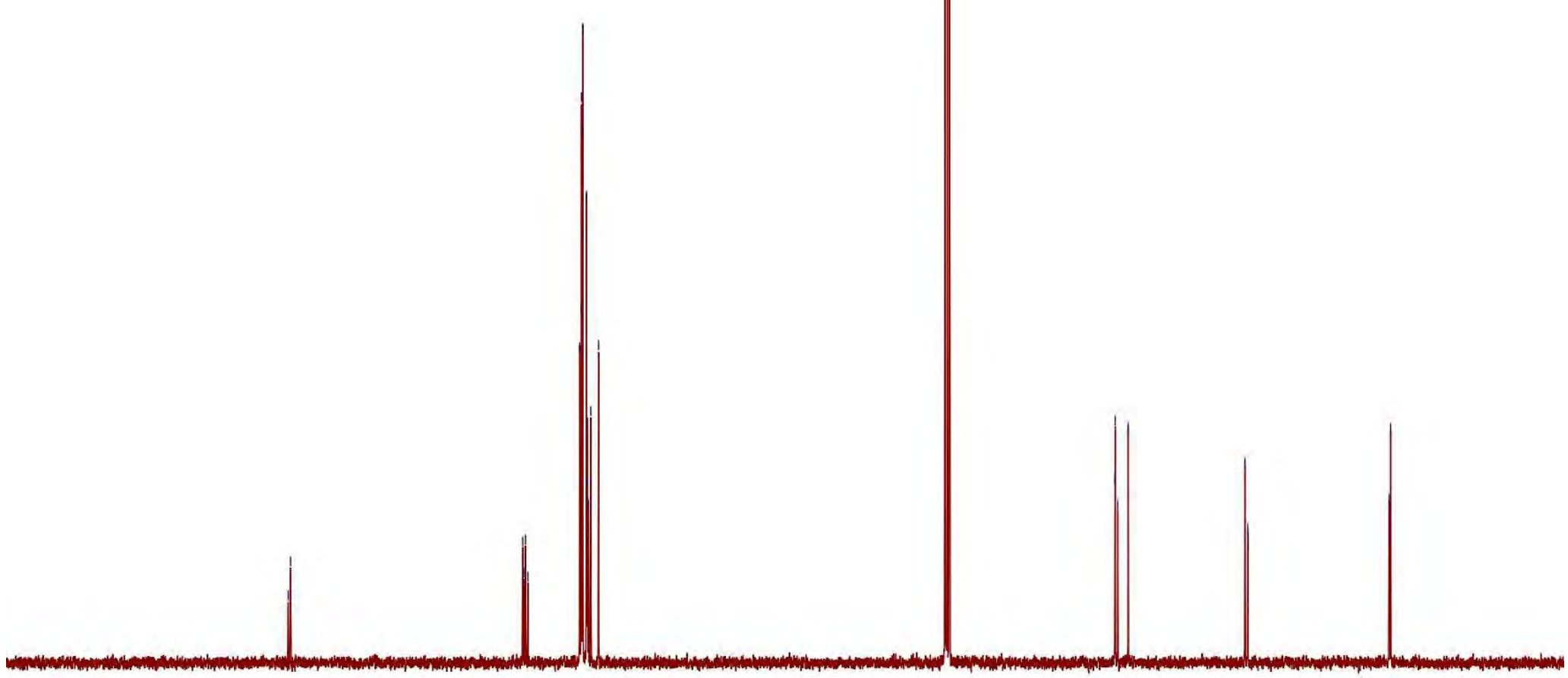

$0 \quad 200 \quad 190$ 
${ }^{1} \mathrm{H} \mathrm{NMR}, 400 \mathrm{MHz}, \mathrm{CDCl}_{3}, 25{ }^{\circ} \mathrm{C}$
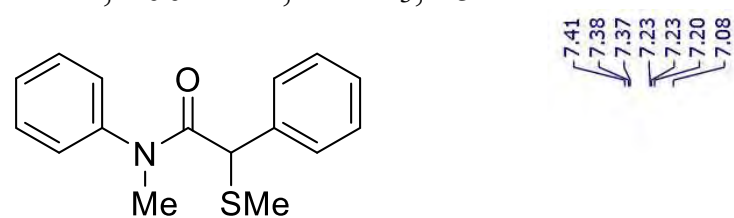

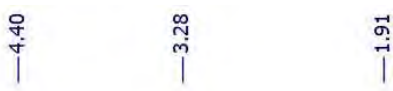

$4 \mathrm{e}$

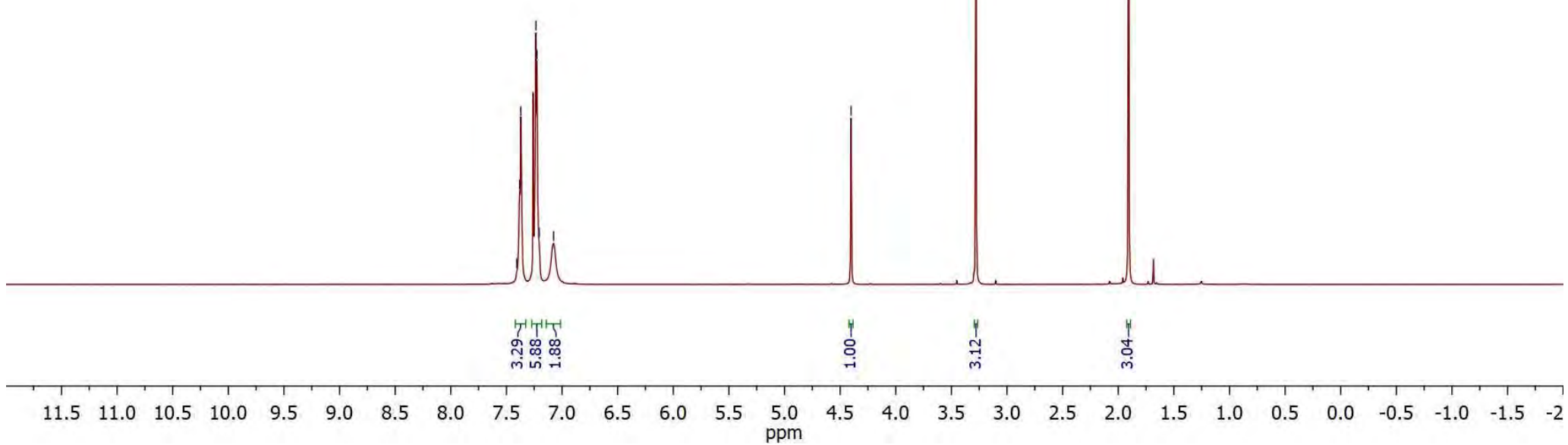


${ }^{13} \mathrm{C} \mathrm{NMR}, 100 \mathrm{MHz}, \mathrm{CDCl}_{3}, 25{ }^{\circ} \mathrm{C}$

ํ. |

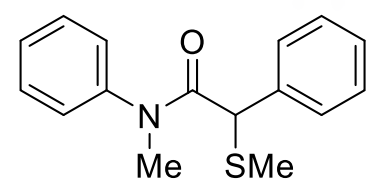

$4 \mathrm{e}$

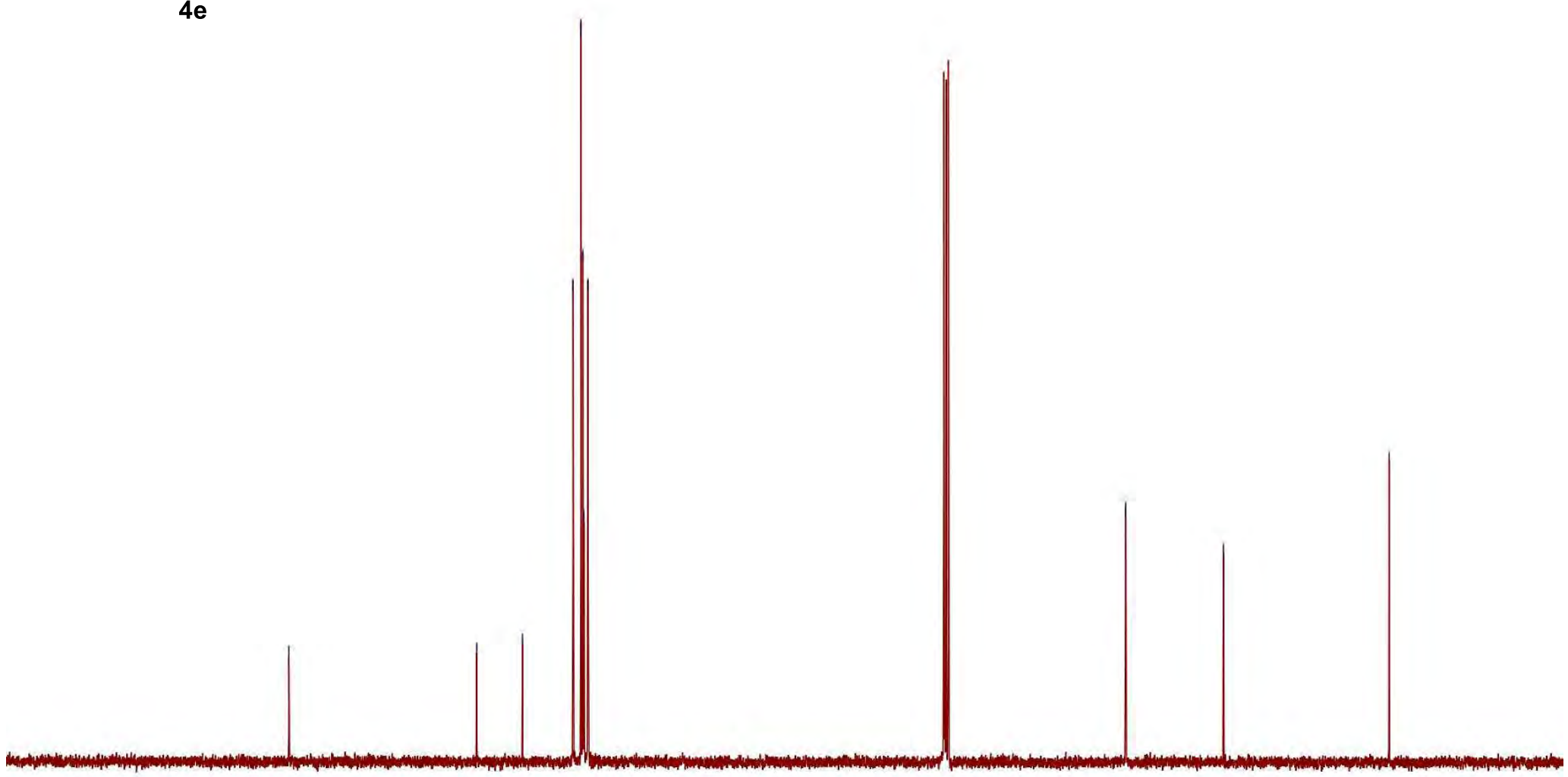

$0 \quad 200 \quad 190$ 
${ }^{1} \mathrm{H} \mathrm{NMR}, 400 \mathrm{MHz}, \mathrm{CDCl}_{3}, 25{ }^{\circ} \mathrm{C}$

$\mathrm{MeO}$

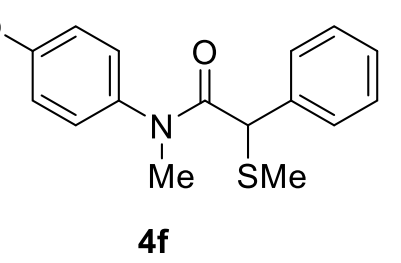

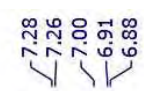

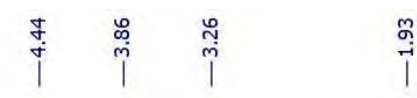

$4 f$

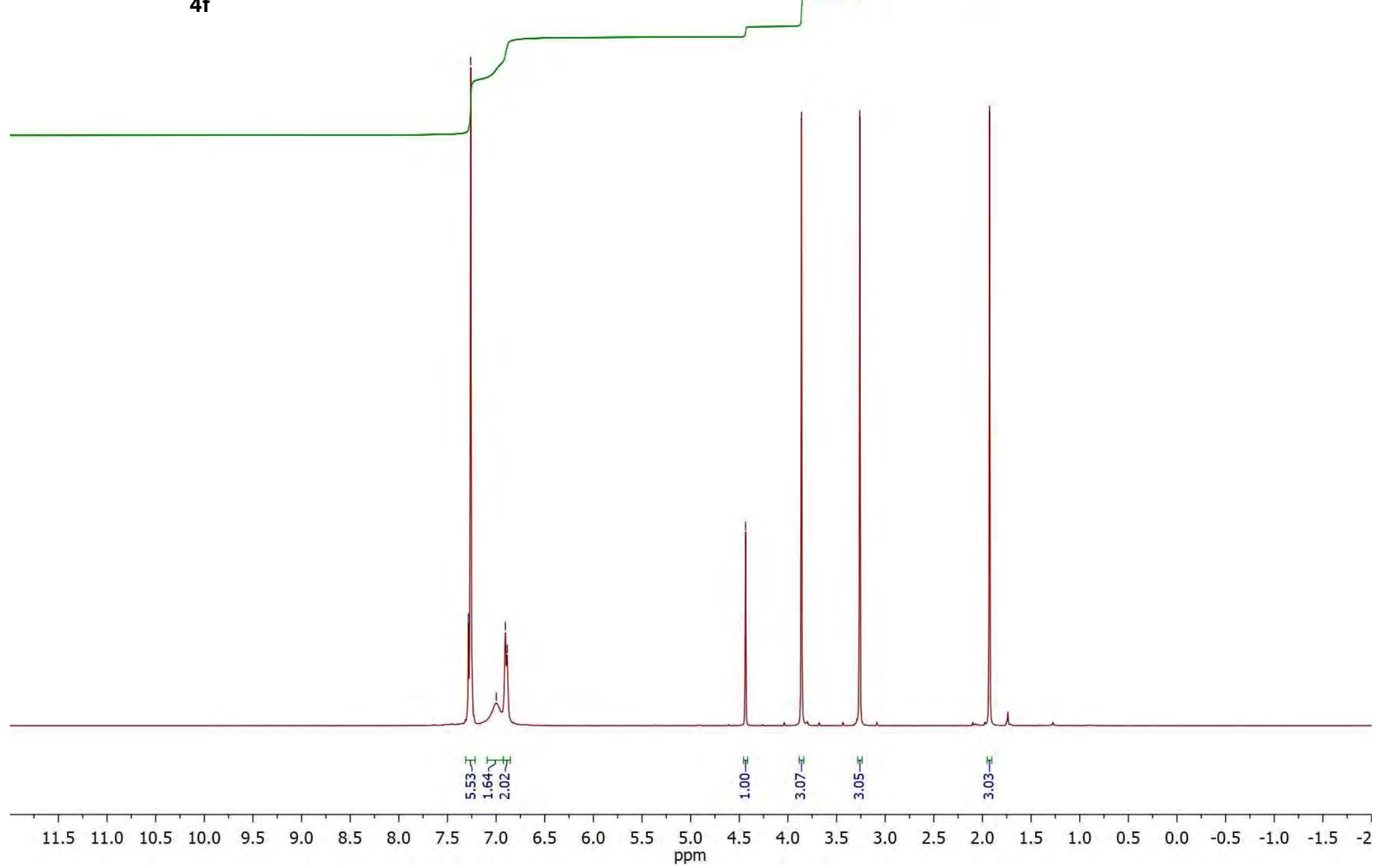


${ }^{13} \mathrm{C} \mathrm{NMR}, 100 \mathrm{MHz}, \mathrm{CDCl}_{3}, 25{ }^{\circ} \mathrm{C}$

$$
\text { | | | }
$$

$\mathrm{MeO}$

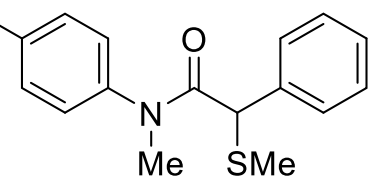

$4 f$

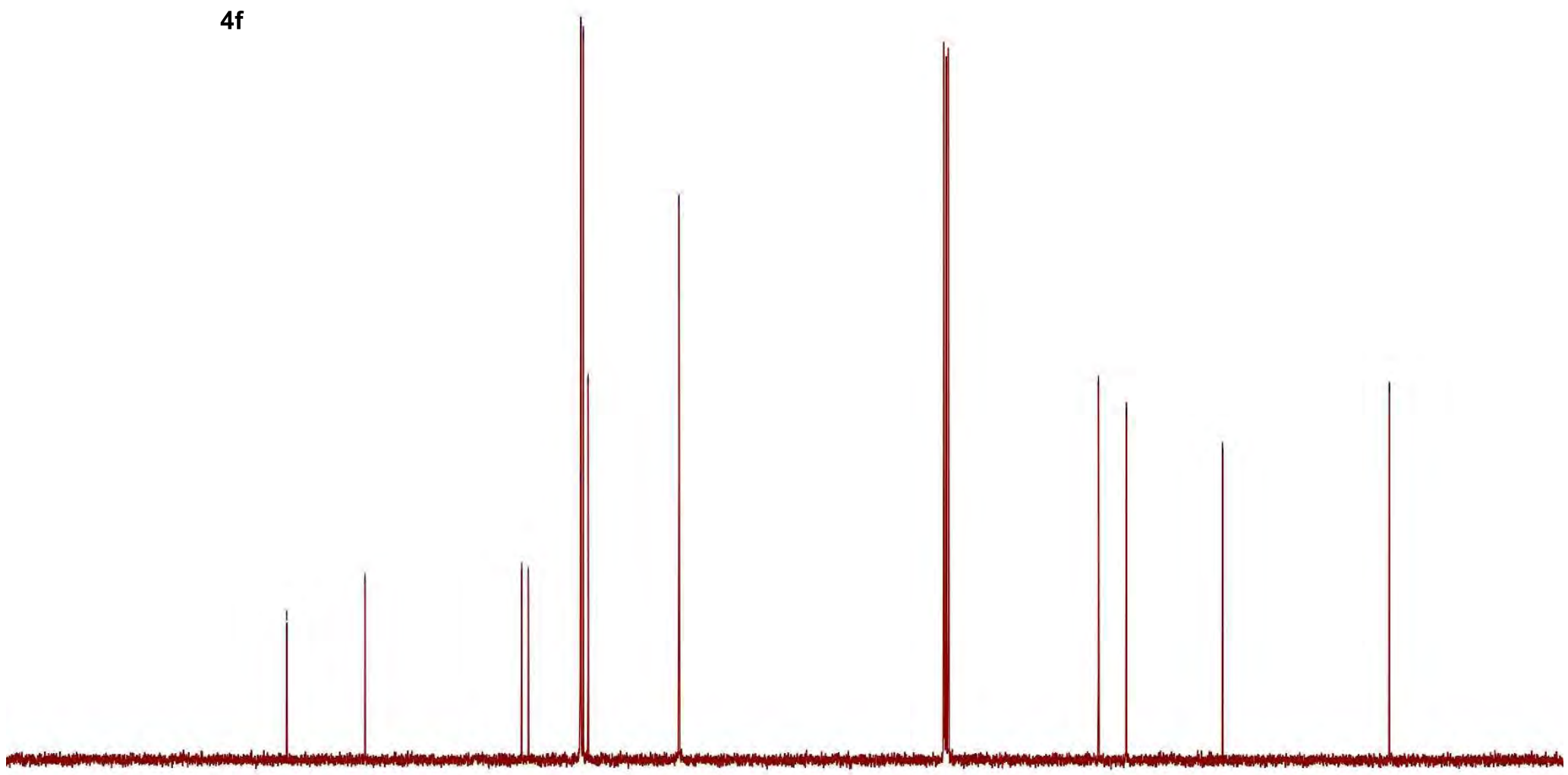

$0 \quad 200 \quad 190$ 
${ }^{1} \mathrm{H} \mathrm{NMR}, 400 \mathrm{MHz}, \mathrm{CDCl}_{3}, 25{ }^{\circ} \mathrm{C}$

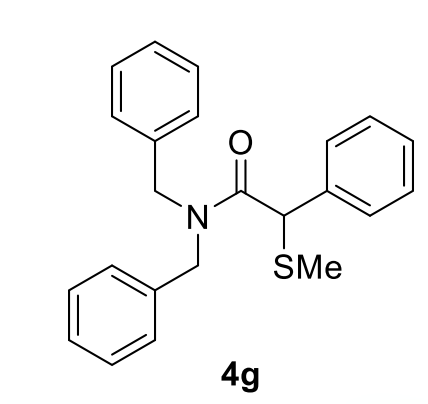

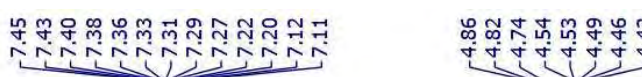

49

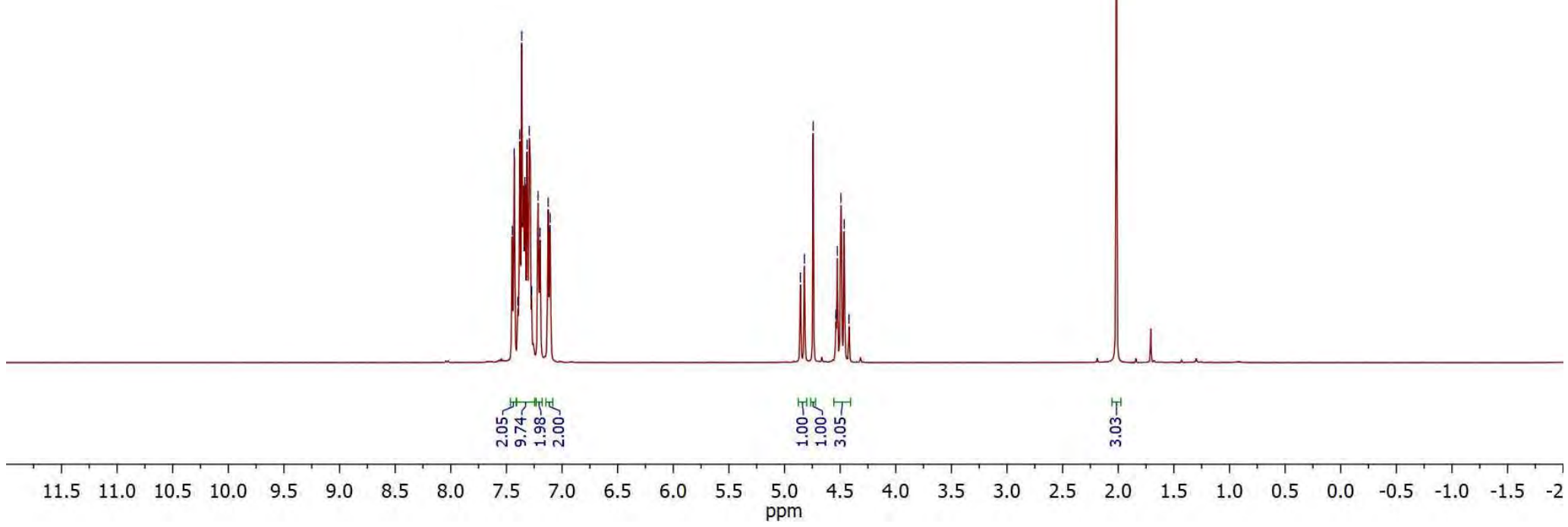


${ }^{13} \mathrm{C} \mathrm{NMR}, 100 \mathrm{MHz}, \mathrm{CDCl}_{3}, 25{ }^{\circ} \mathrm{C}$
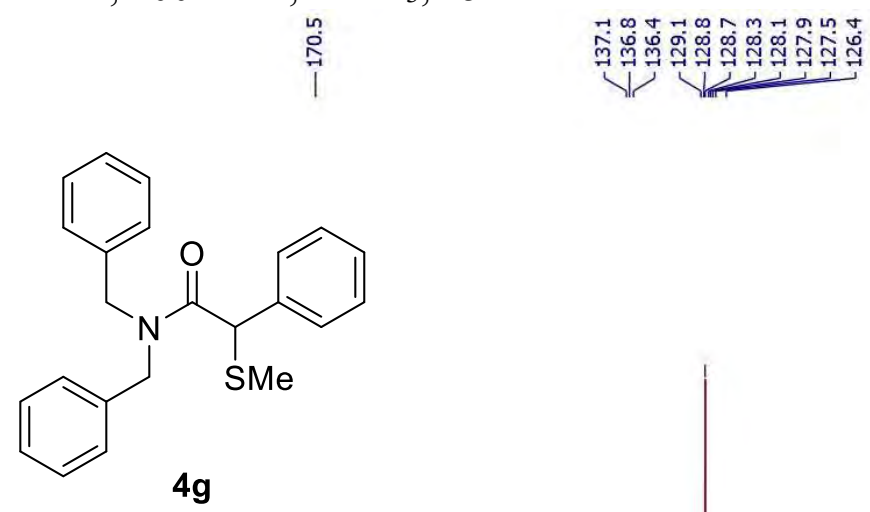

$4 \mathrm{~g}$

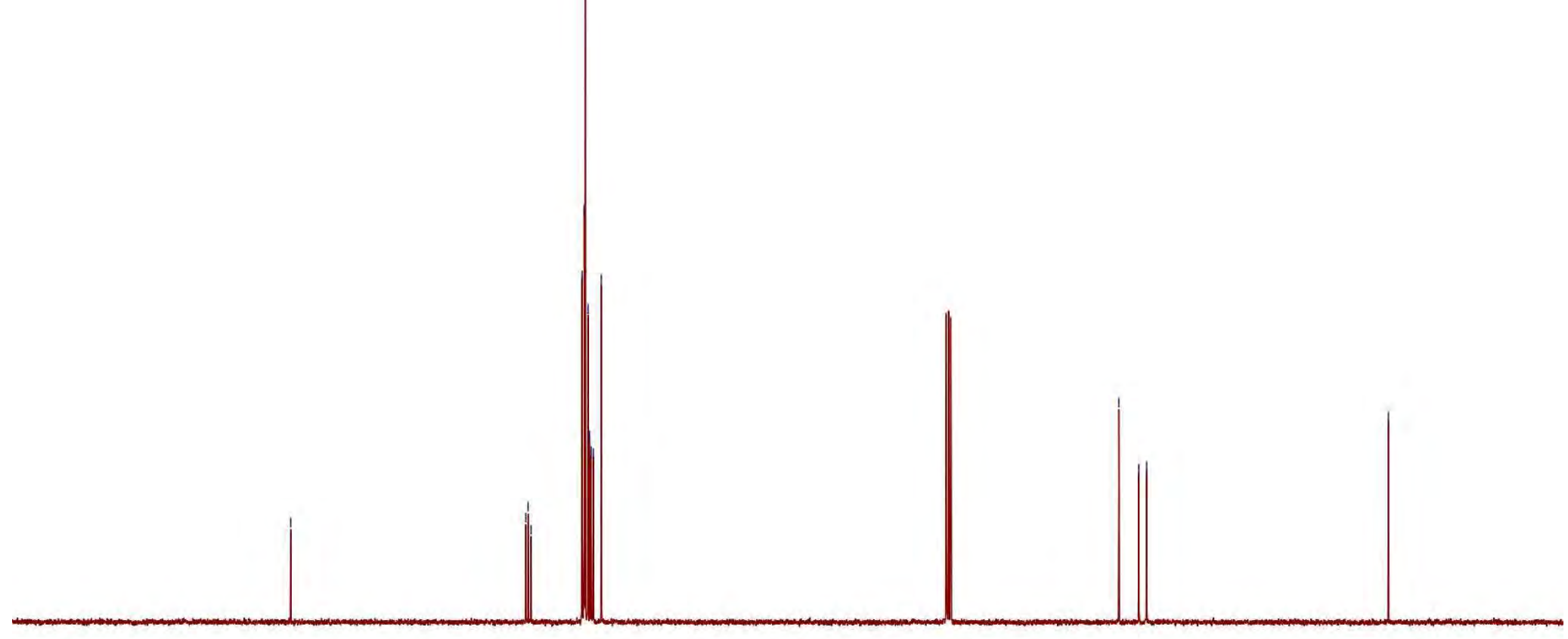

$10 \quad 200 \quad 190$ $-1$ 
${ }^{1} \mathrm{H} \mathrm{NMR}, 400 \mathrm{MHz}, \mathrm{CDCl}_{3}, 25{ }^{\circ} \mathrm{C}$

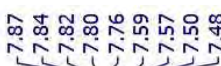

|<smiles>CSC(C(=O)N1CCOCC1)c1ccc2ccccc2c1</smiles>

4h
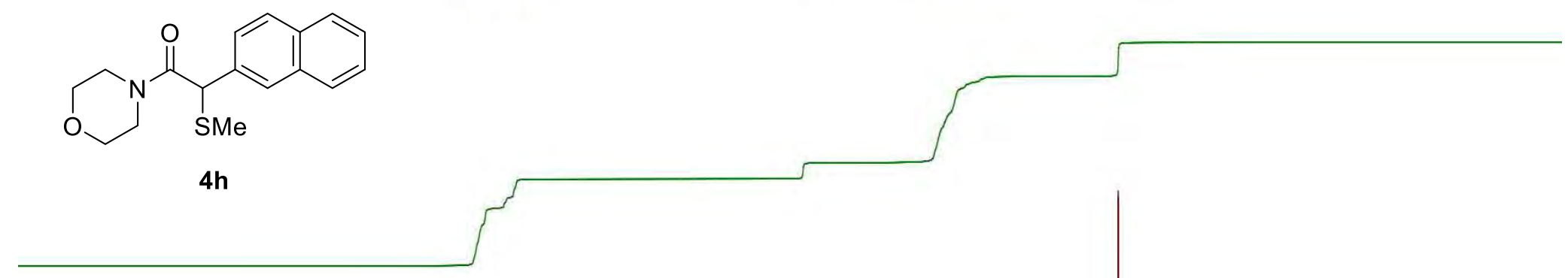
${ }^{13} \mathrm{C} \mathrm{NMR}, 100 \mathrm{MHz}, \mathrm{CDCl}_{3}, 25{ }^{\circ} \mathrm{C}$

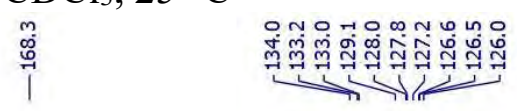

产

$\stackrel{\substack{i \\ i}}{i}$

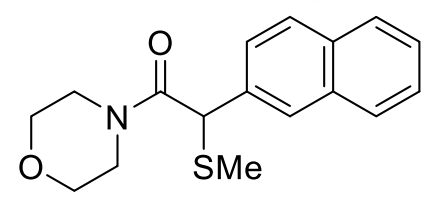

$4 \mathrm{~h}$

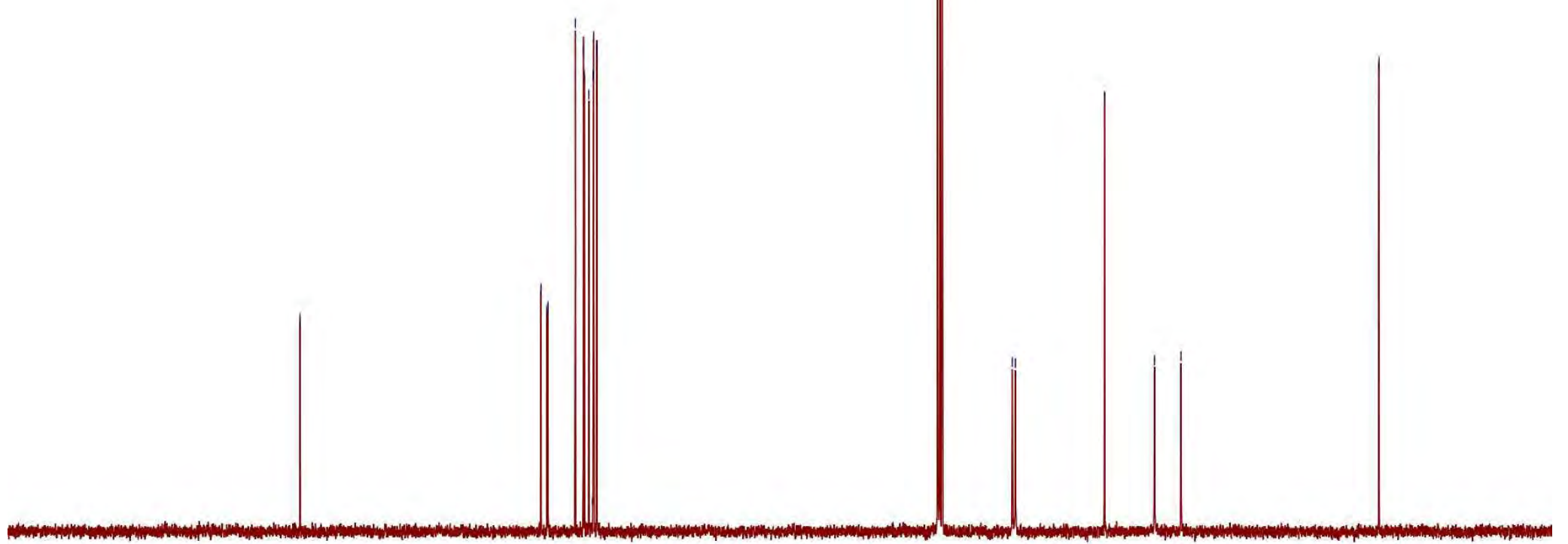

$\begin{array}{llll}0 & 200 & 190 & 180\end{array}$

160

150

130

120

100

$90 \quad 80$

70

$60 \quad 50$

40

$30 \quad 20$

10 
${ }^{1} \mathrm{H}$ NMR, $400 \mathrm{MHz}, \mathrm{CDCl}_{3}, 25^{\circ} \mathrm{C}$
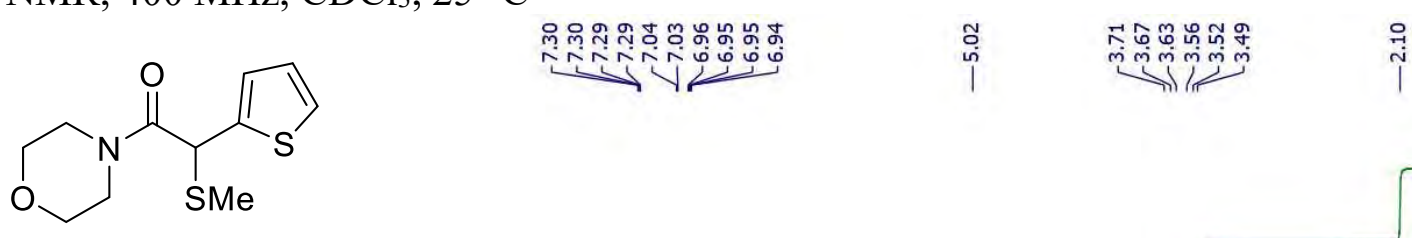

$4 \mathbf{i}$

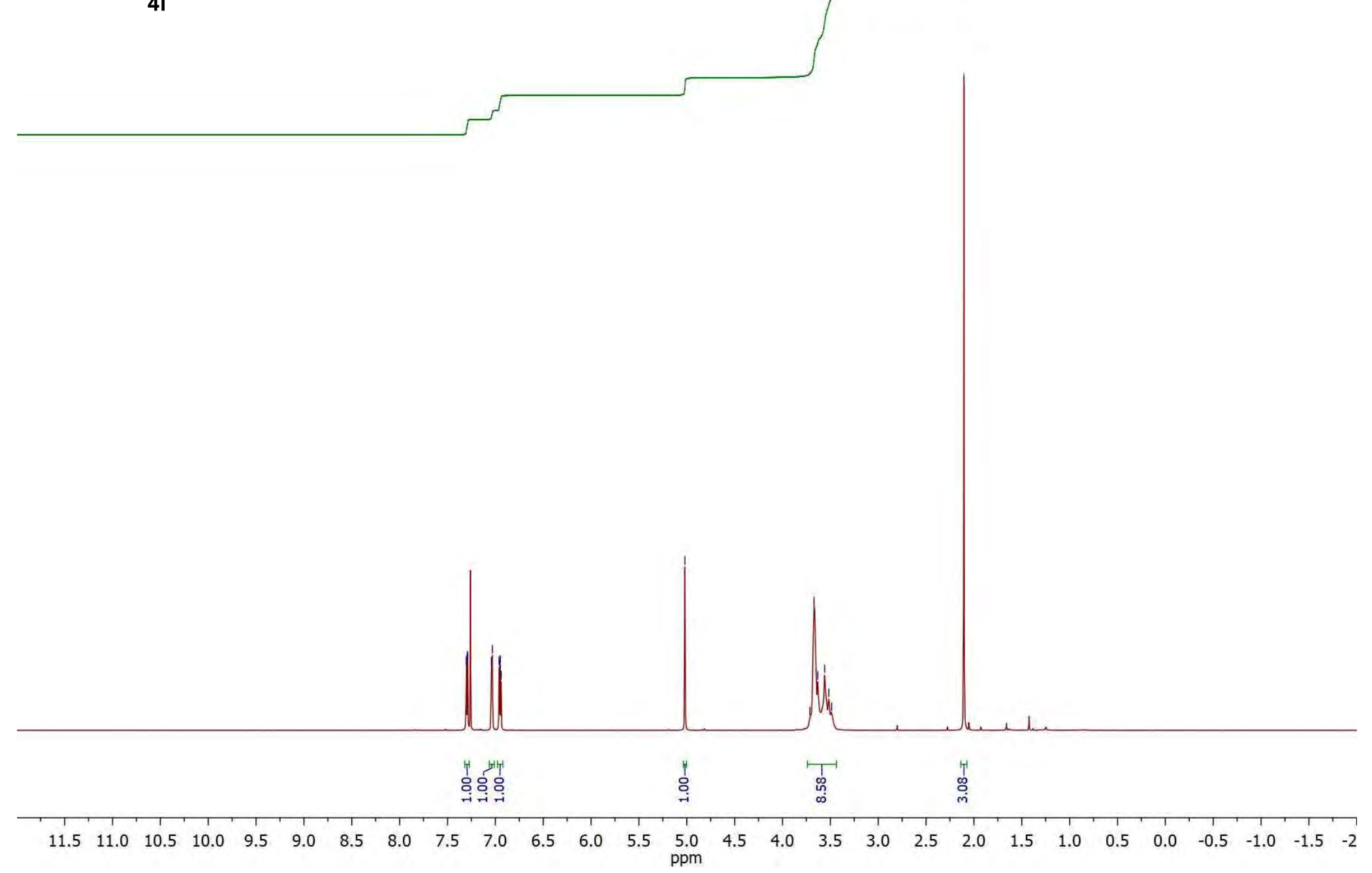


${ }^{13} \mathrm{C}$ NMR, $100 \mathrm{MHz}, \mathrm{CDCl}_{3}, 25{ }^{\circ} \mathrm{C}$
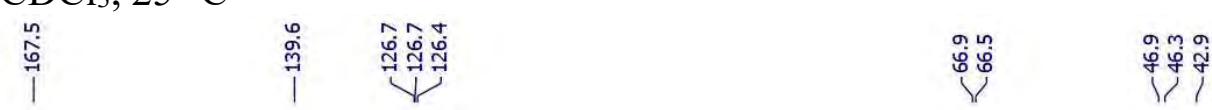

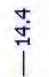

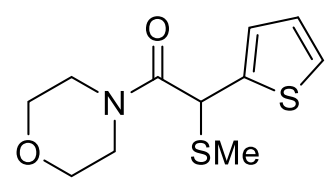

$4 \mathbf{i}$

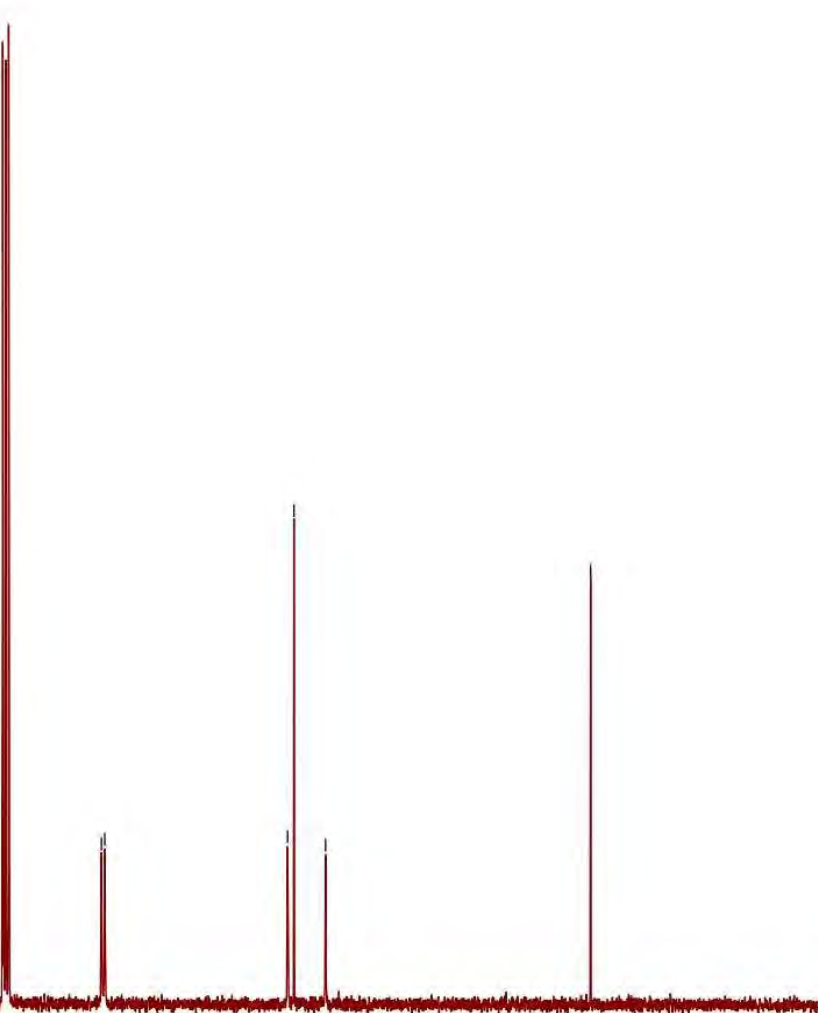

$0 \quad 200 \quad 190$ 
${ }^{1} \mathrm{H} \mathrm{NMR}, 400 \mathrm{MHz}, \mathrm{CDCl}_{3}, 25^{\circ} \mathrm{C}$

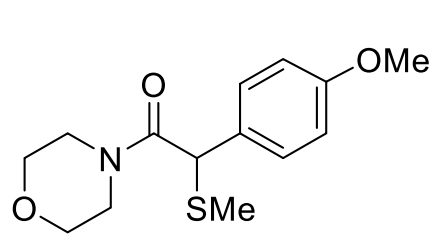

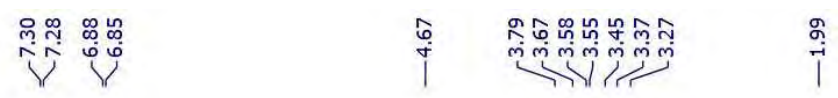

4j

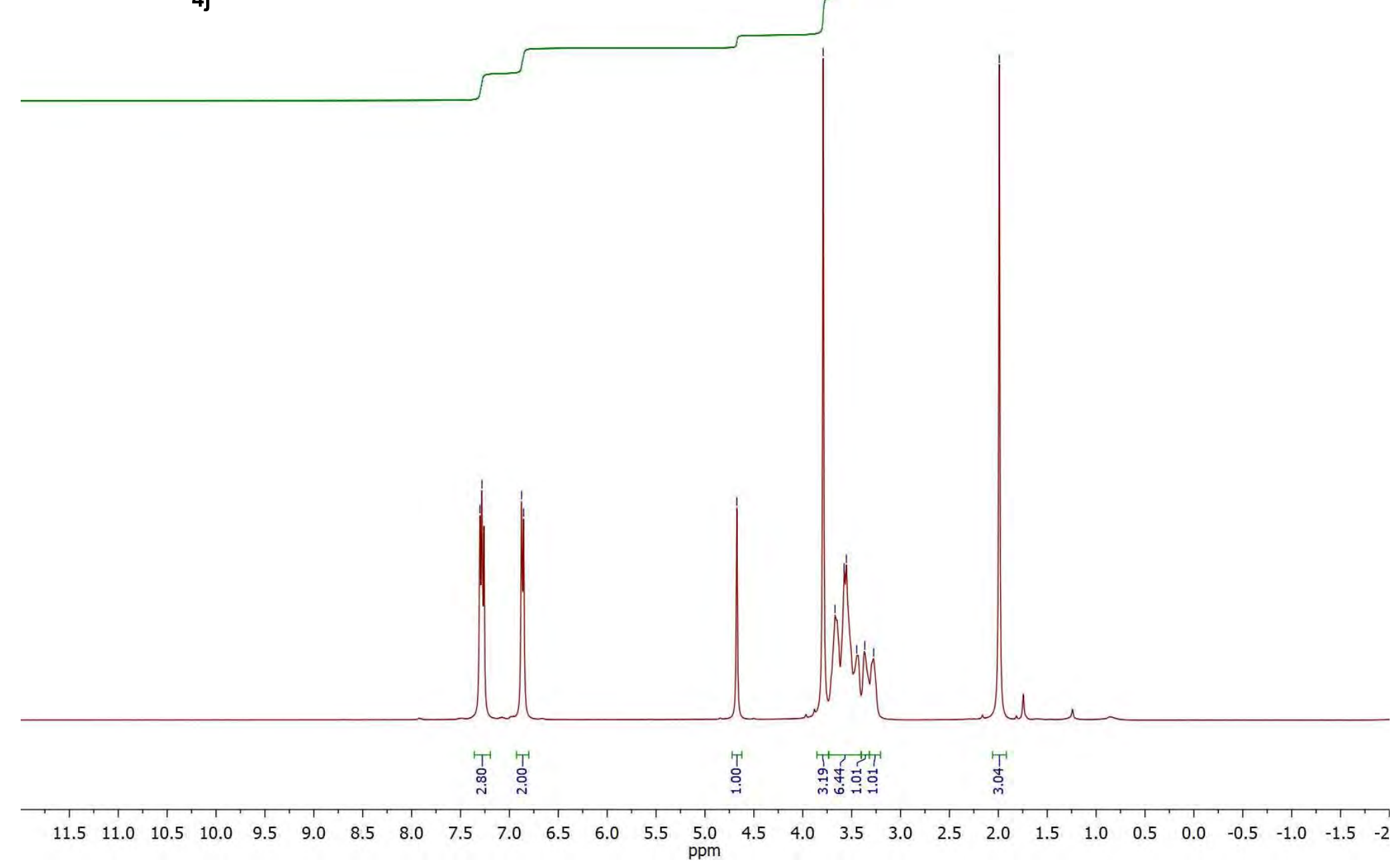


${ }^{13} \mathrm{C} \mathrm{NMR}, 100 \mathrm{MHz}, \mathrm{CDCl}_{3}, 25{ }^{\circ} \mathrm{C}$ ।

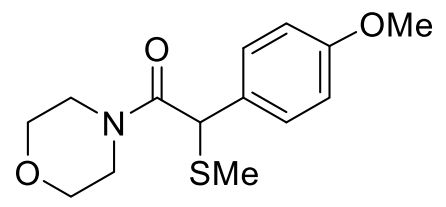

$4 \mathbf{j}$

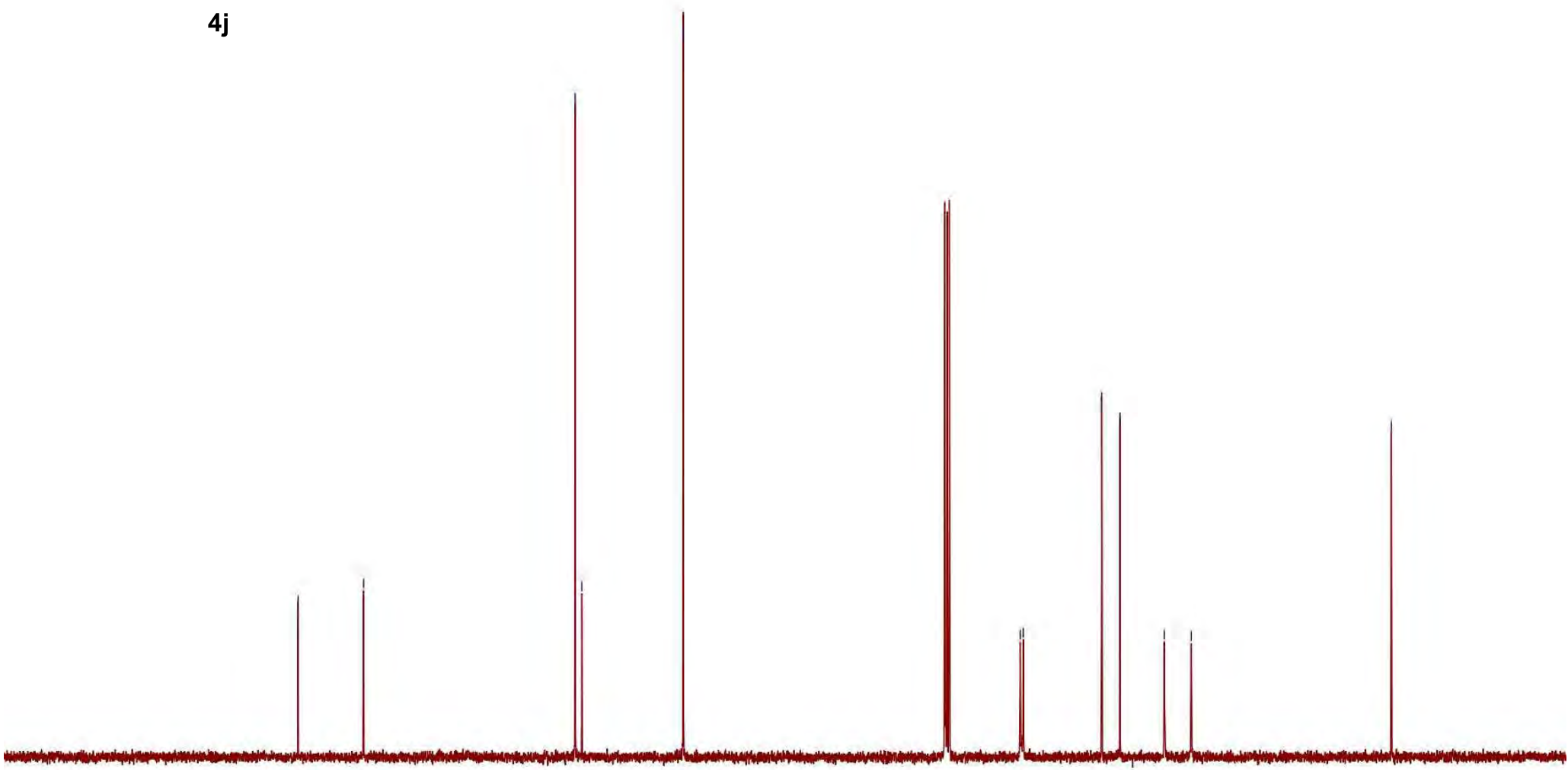

$.0 \quad 200 \quad 190$ 
${ }^{1} \mathrm{H} \mathrm{NMR}, 400 \mathrm{MHz}, \mathrm{CDCl}_{3}, 25^{\circ} \mathrm{C}$
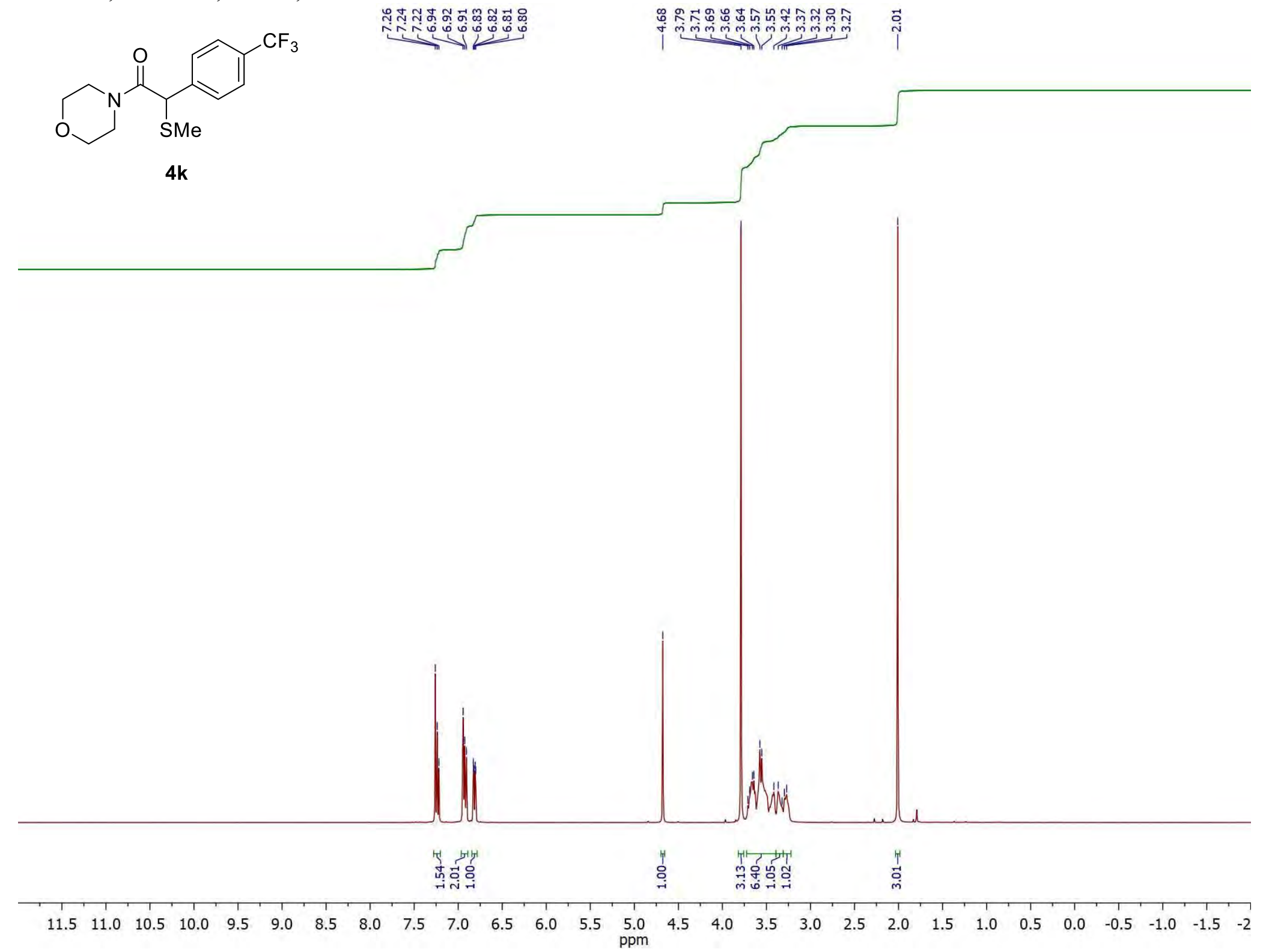
Chemoselective $\alpha$-Sulfidation of Amides Using Sulfoxide Reagents

M. Leypold, K. A. D’Angelo and M. Movassaghi

Page S144 / S183

${ }^{13} \mathrm{C} \mathrm{NMR,} 100 \mathrm{MHz}, \mathrm{CDCl}_{3}, 25{ }^{\circ} \mathrm{C}$

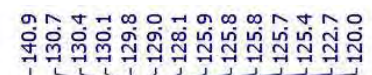

帒

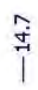<smiles>CC(C(=O)N1CCOCC1)c1ccc(C(F)(F)F)cc1</smiles>

$4 k$

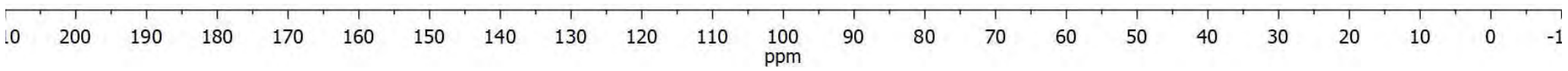


${ }^{19} \mathrm{~F} \mathrm{NMR,} 376 \mathrm{MHz}, \mathrm{CDCl}_{3}, 25^{\circ} \mathrm{C}$
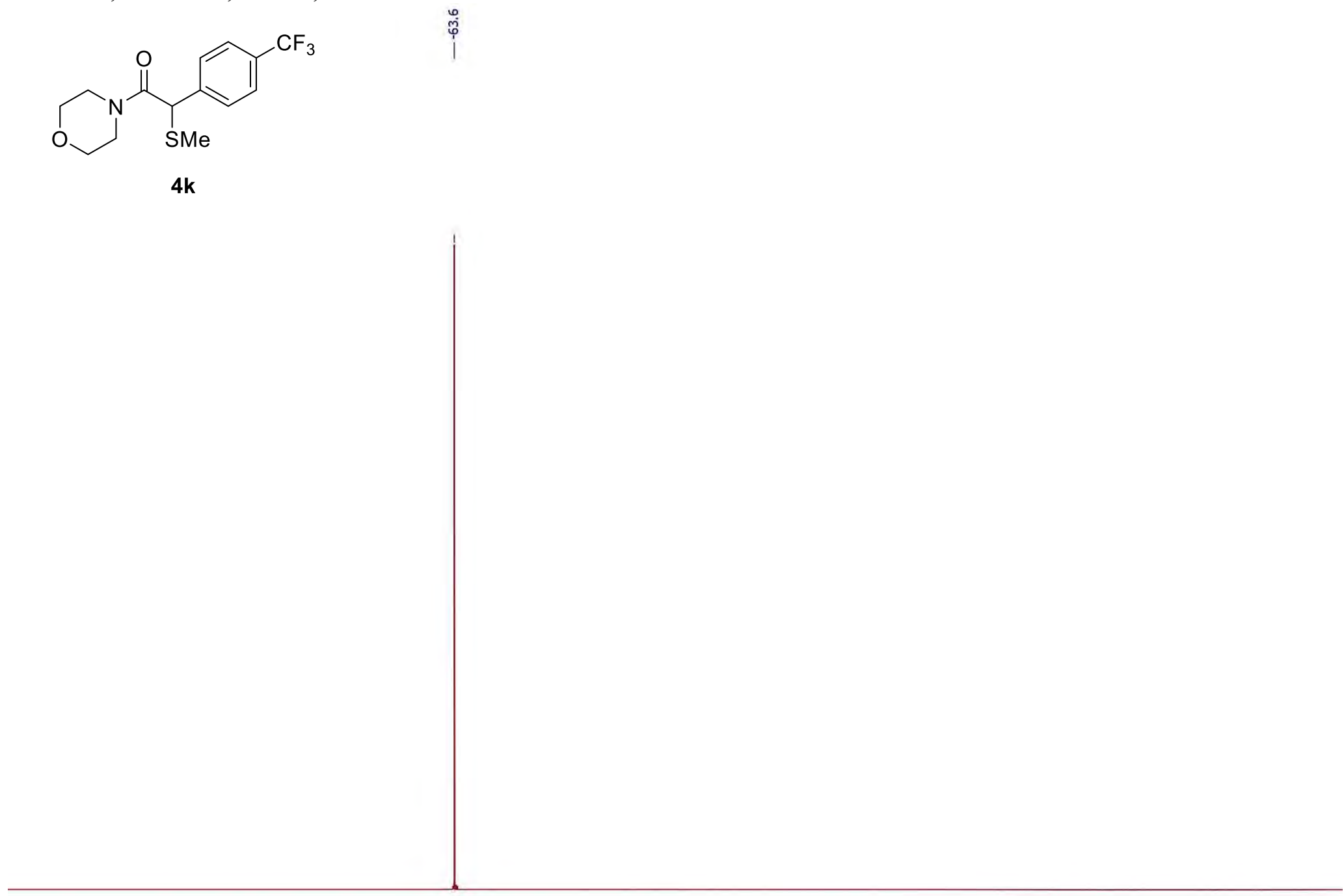

\begin{tabular}{|c|c|c|c|c|c|c|c|c|c|c|c|c|c|c|c|c|c|c|c|c|}
\hline 0 & $\begin{array}{c}1 \\
-10\end{array}$ & $\begin{array}{l}1 \\
-20\end{array}$ & $\begin{array}{l}1 \\
-30\end{array}$ & $\begin{array}{l}1 \\
-40\end{array}$ & $\begin{array}{c}1 \\
-50\end{array}$ & $\begin{array}{c}1 \\
-60\end{array}$ & $\begin{array}{l}1 \\
-70\end{array}$ & -80 & $\begin{array}{l}1 \\
-90\end{array}$ & $\begin{array}{c}1 \\
-100 \\
\mathrm{ppm}\end{array}$ & $\begin{array}{c}1 \\
-110\end{array}$ & $\begin{array}{c}1 \\
-120\end{array}$ & $\begin{array}{c}1 \\
-130\end{array}$ & -140 & $\begin{array}{c}1 \\
-150\end{array}$ & $\begin{array}{c}1 \\
-160\end{array}$ & $\begin{array}{c}1 \\
-170\end{array}$ & -180 & -190 & $\begin{array}{c}1 \\
-200\end{array}$ \\
\hline
\end{tabular}


${ }^{1} \mathrm{H} \mathrm{NMR}, 400 \mathrm{MHz}, \mathrm{CDCl}_{3}, 25^{\circ} \mathrm{C}$

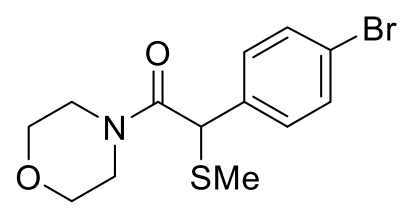

41

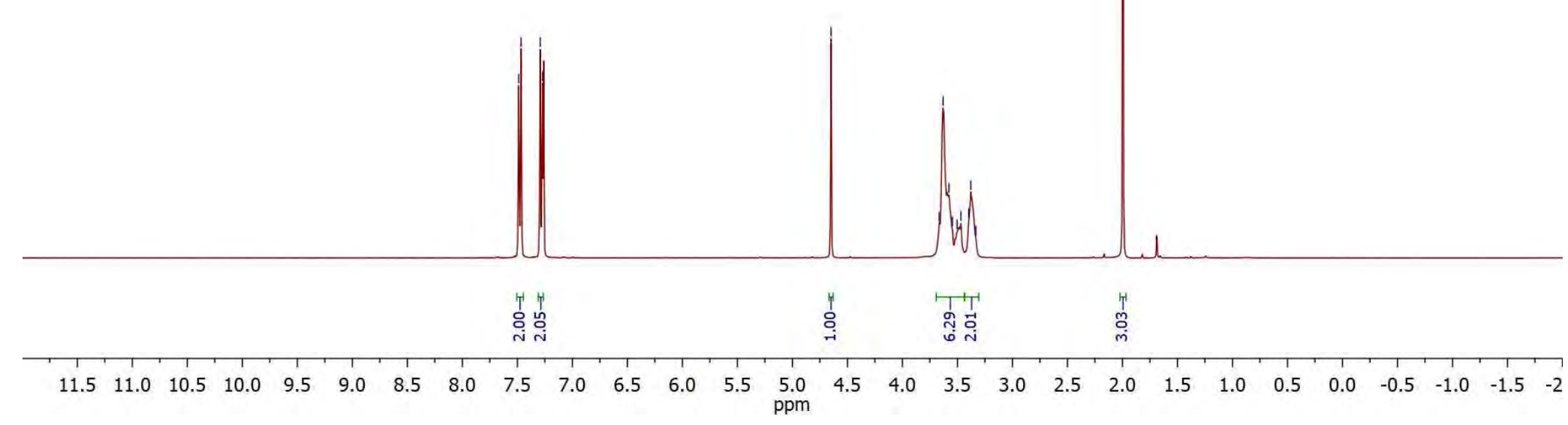


${ }^{13} \mathrm{C}$ NMR, $100 \mathrm{MHz}, \mathrm{CDCl}_{3}, 25{ }^{\circ} \mathrm{C}$

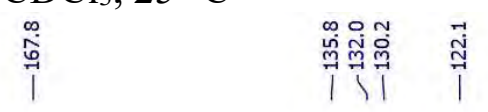

Vll

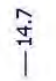

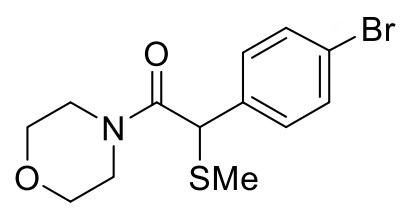

4

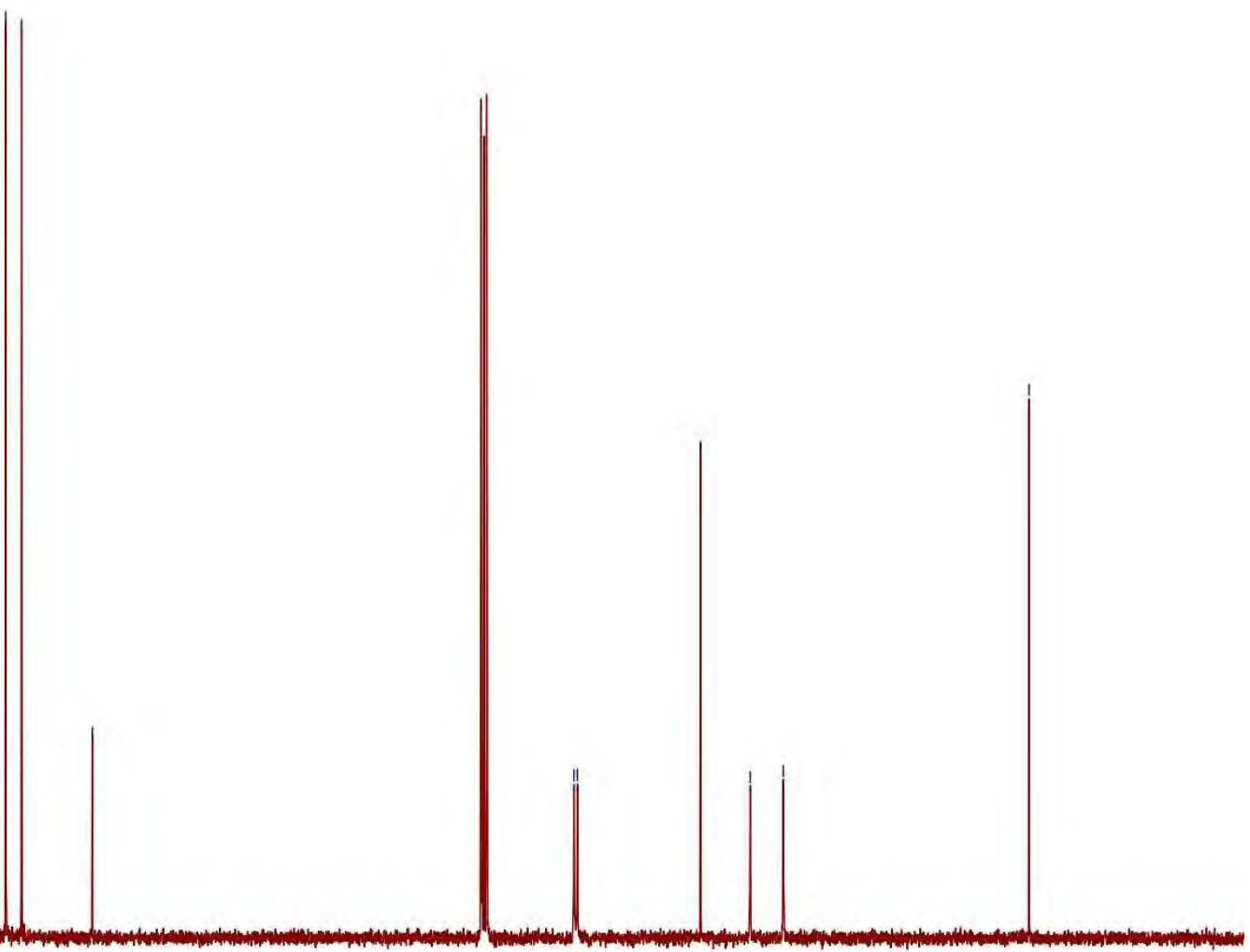

$0 \quad 200-190$ 
Chemoselective $\alpha$-Sulfidation of Amides Using Sulfoxide Reagents

M. Leypold, K. A. D’Angelo and M. Movassaghi

Page S148 / S183

${ }^{1} \mathrm{H} \mathrm{NMR}, 400 \mathrm{MHz}, \mathrm{CDCl}_{3}, 25^{\circ} \mathrm{C}$

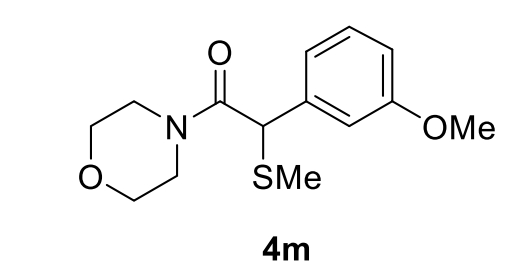

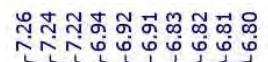

gog

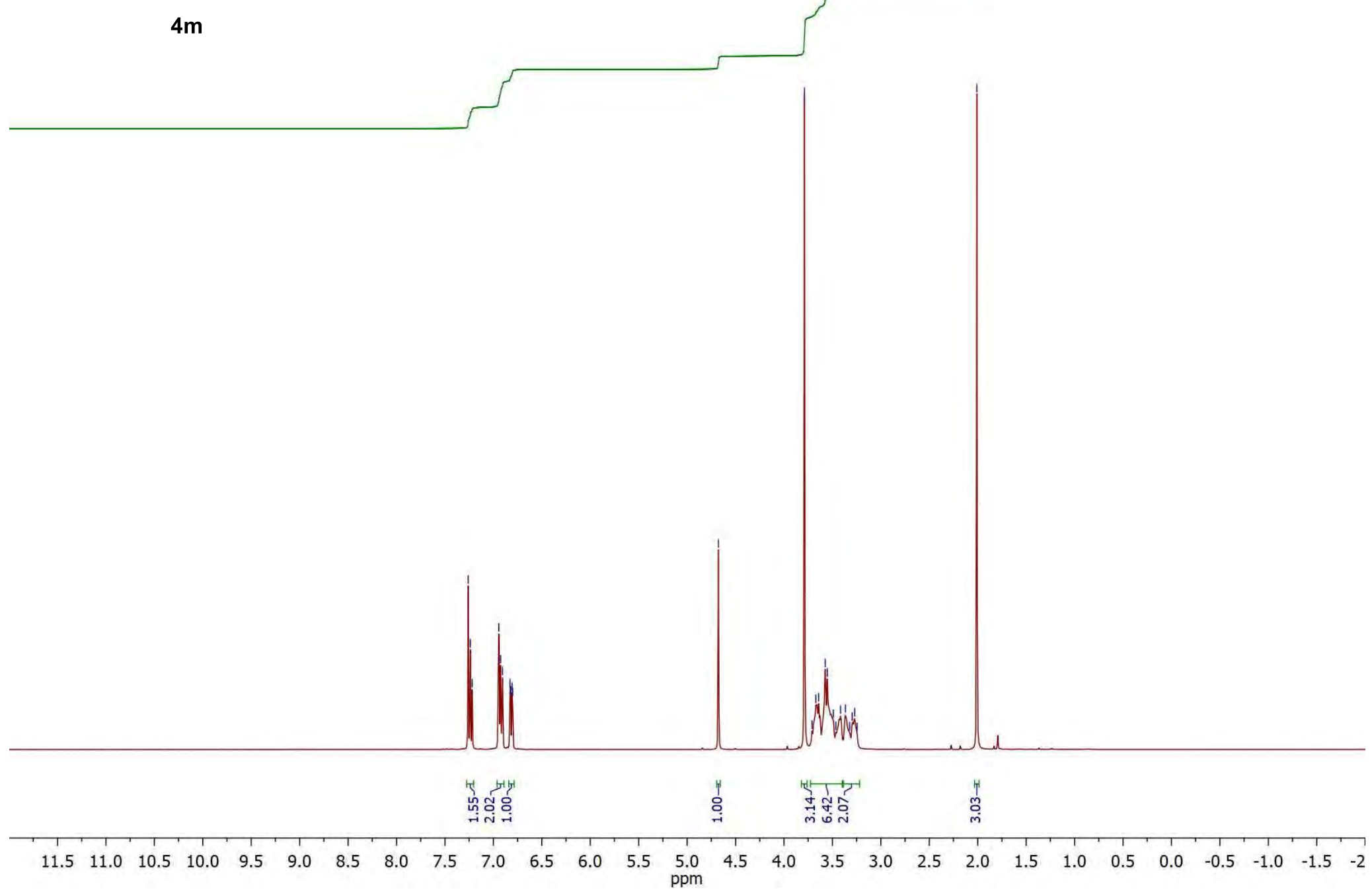


${ }^{13} \mathrm{C} \mathrm{NMR}, 100 \mathrm{MHz}, \mathrm{CDCl}_{3}, 25{ }^{\circ} \mathrm{C}$

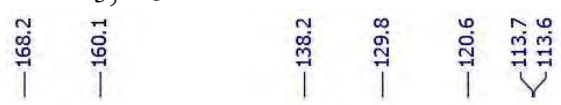

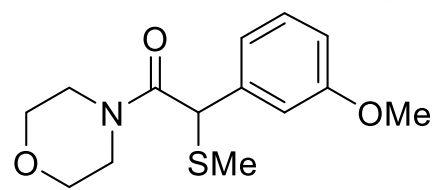

$4 m$

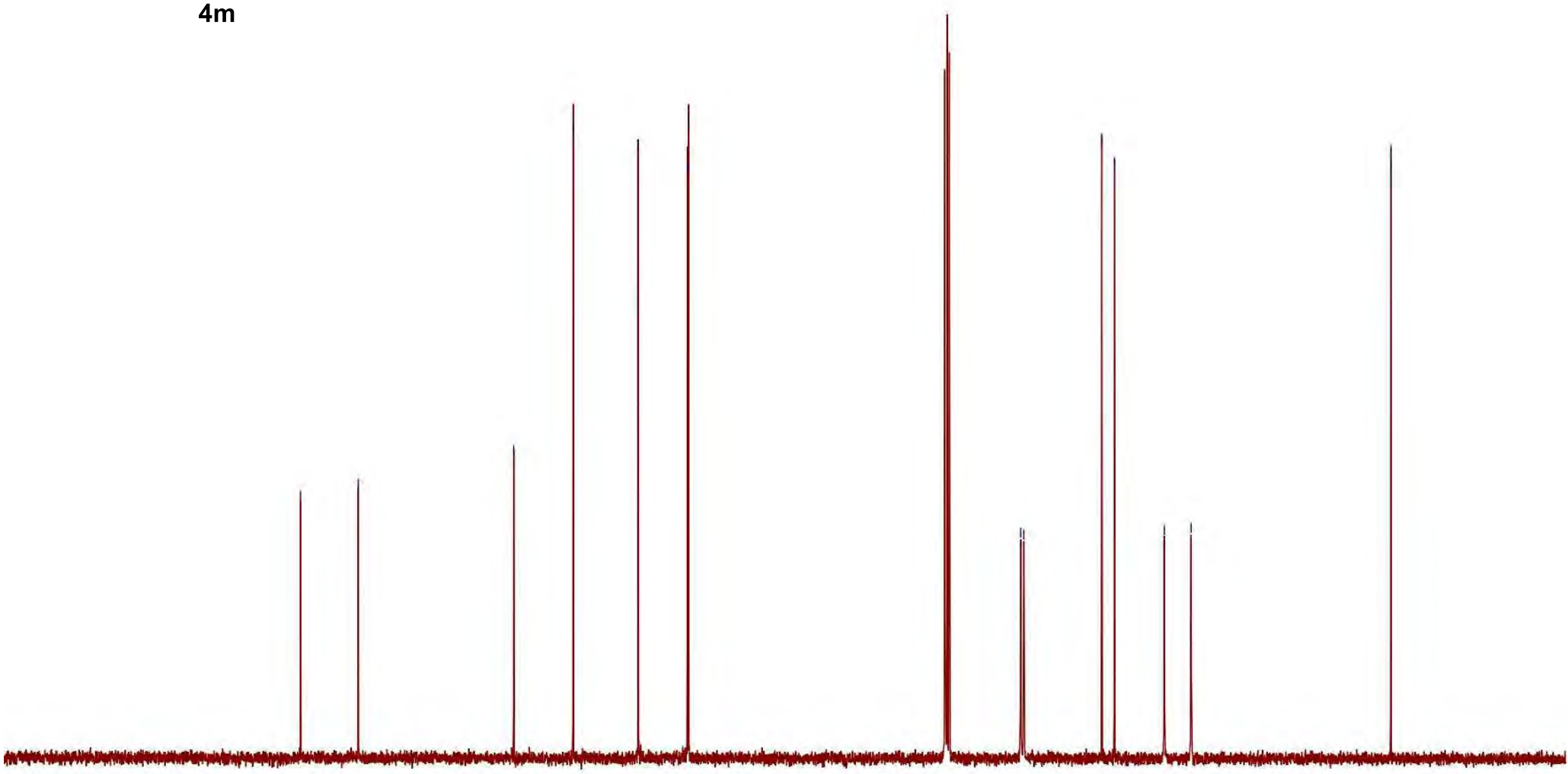

$\begin{array}{llll}0 & 200 & 190 & 180\end{array}$

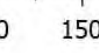

120 
${ }^{1} \mathrm{H} \mathrm{NMR}, 400 \mathrm{MHz}, \mathrm{CDCl}_{3}, 25^{\circ} \mathrm{C}$

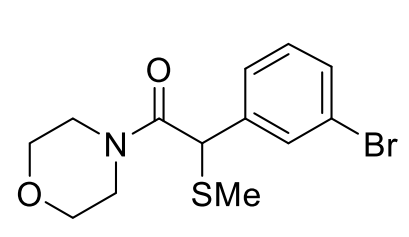

$4 n$

\section{$\mathrm{Br}$}
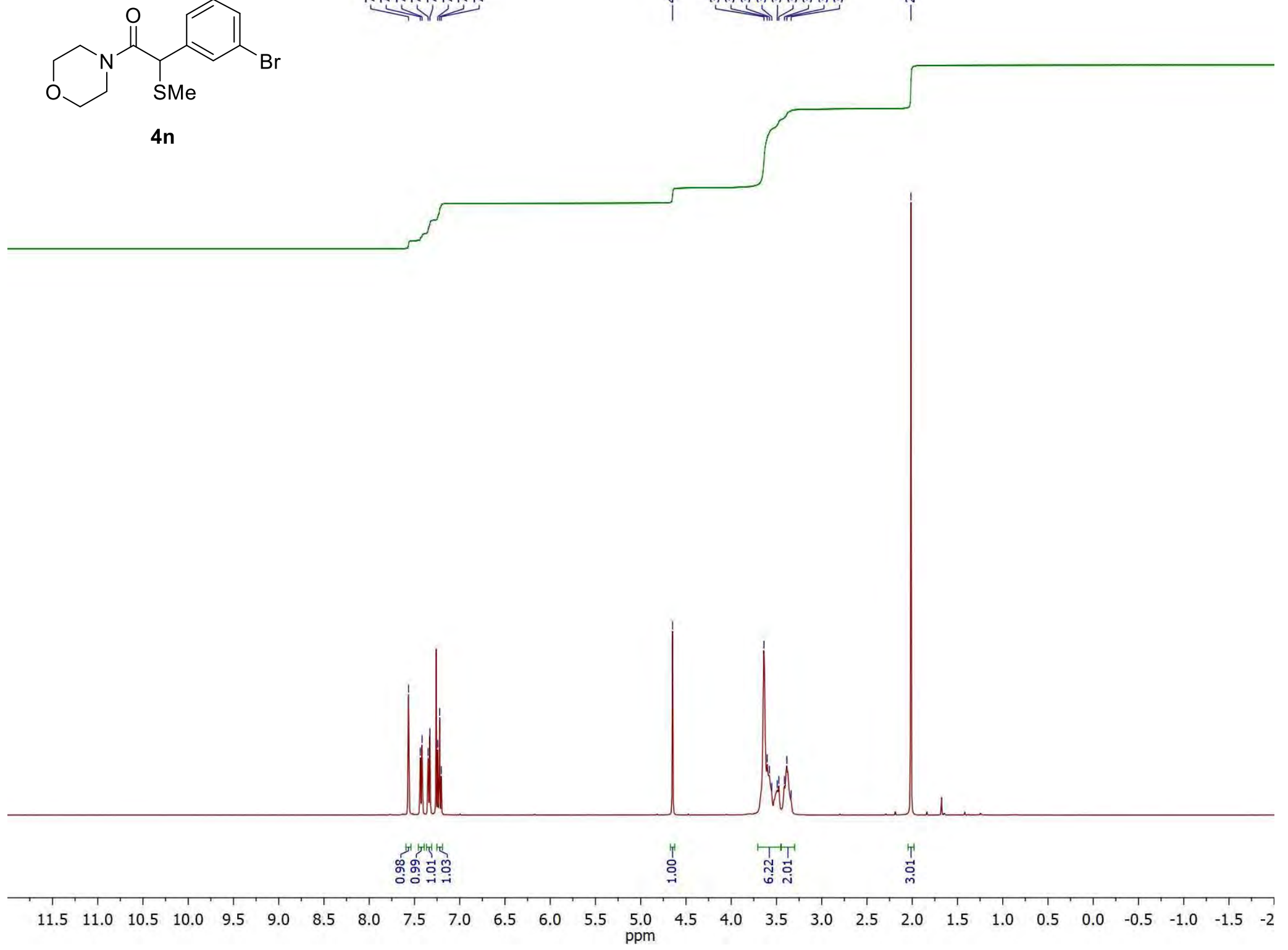
${ }^{13} \mathrm{C} \mathrm{NMR,} 100 \mathrm{MHz}, \mathrm{CDCl}_{3}, 25{ }^{\circ} \mathrm{C}$
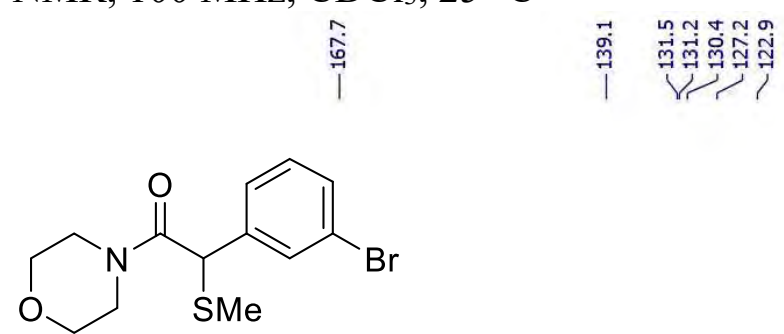

$4 n$

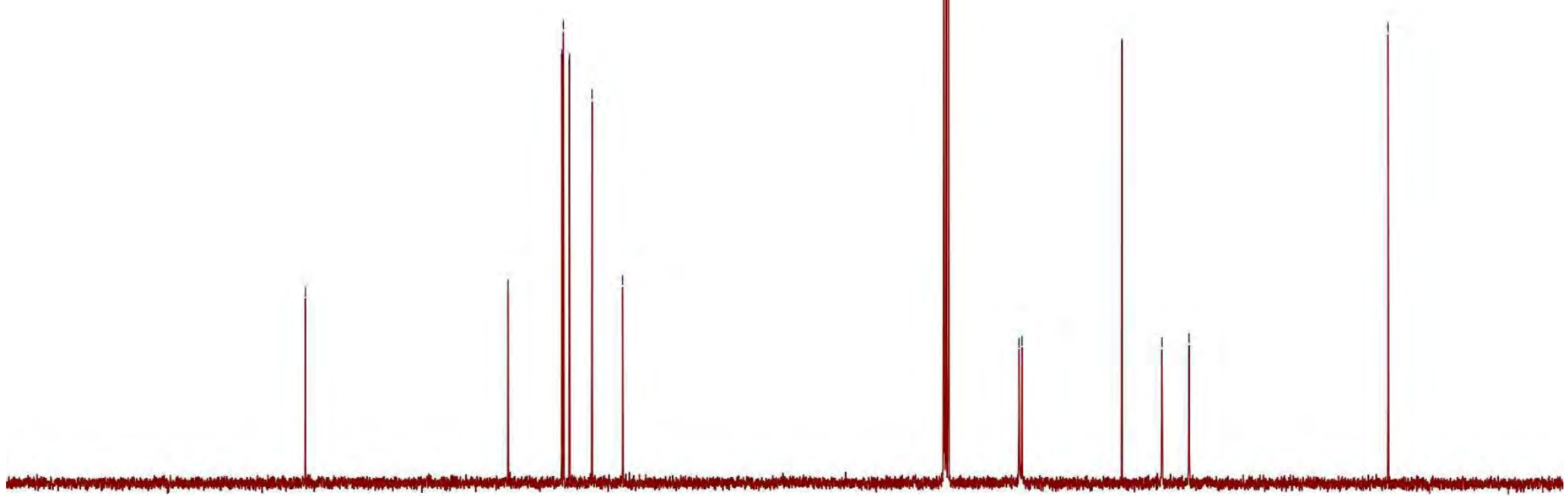

$0 \quad 200 \quad 190$ 
${ }^{1} \mathrm{H} \mathrm{NMR}, 400 \mathrm{MHz}, \mathrm{CDCl}_{3}, 25^{\circ} \mathrm{C}$

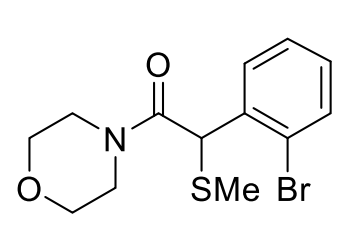

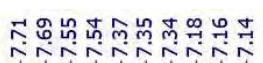

|

40

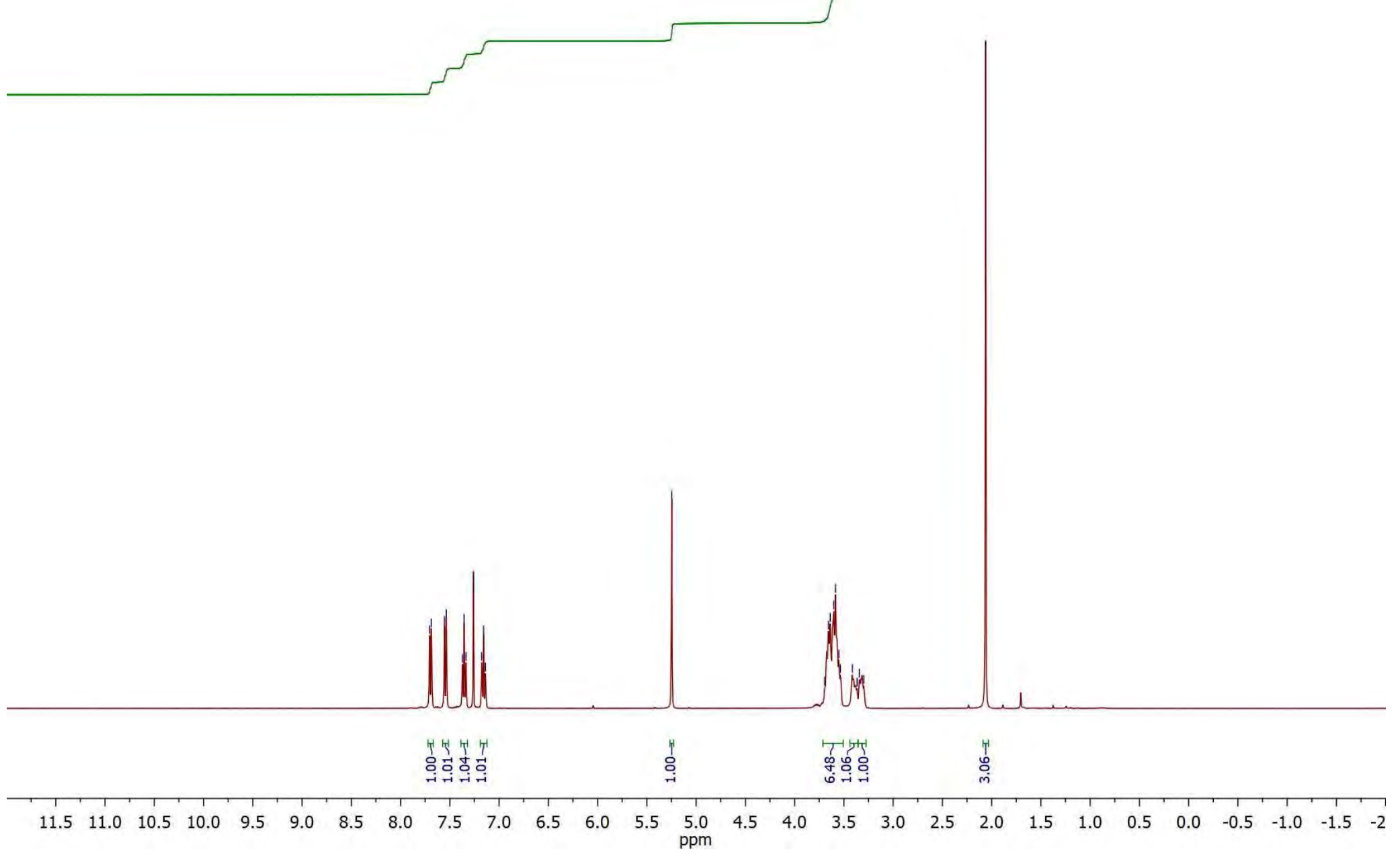


${ }^{13} \mathrm{C} \mathrm{NMR}, 100 \mathrm{MHz}, \mathrm{CDCl}_{3}, 25{ }^{\circ} \mathrm{C}$

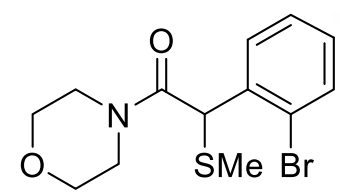

40

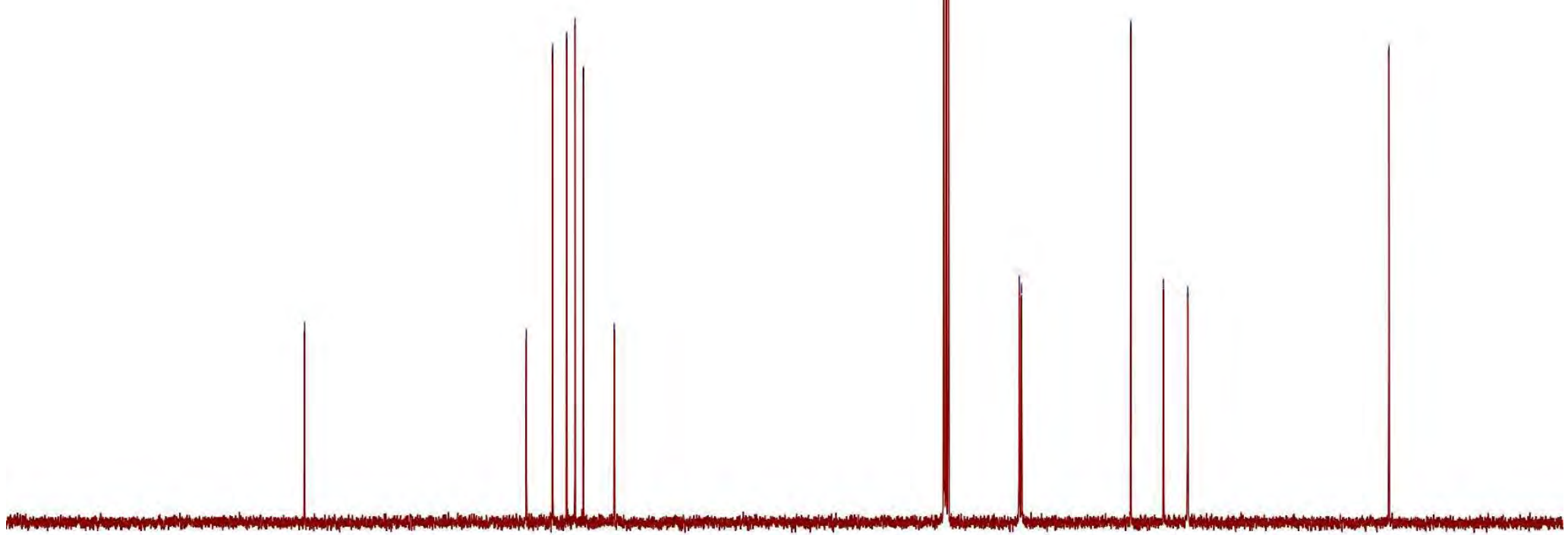

$\begin{array}{llll}0 & 200 \quad 190 \quad 180 & 170\end{array}$

160

$150 \quad 140$

130

120 
${ }^{1} \mathrm{H} \mathrm{NMR}, 400 \mathrm{MHz}, \mathrm{CDCl}_{3}, 25^{\circ} \mathrm{C}$
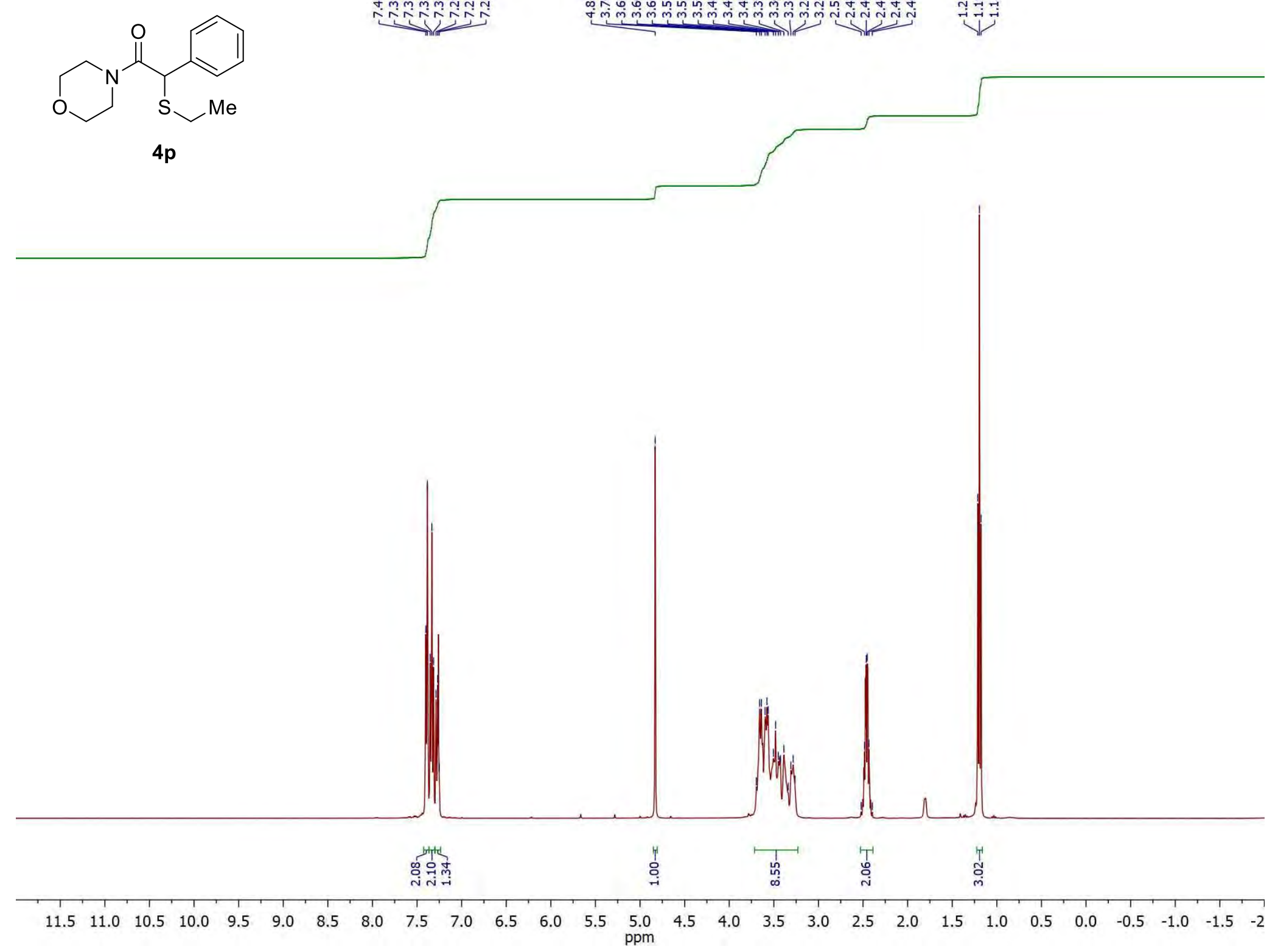
${ }^{13} \mathrm{C}$ NMR, $100 \mathrm{MHz}, \mathrm{CDCl}_{3}, 25{ }^{\circ} \mathrm{C}$
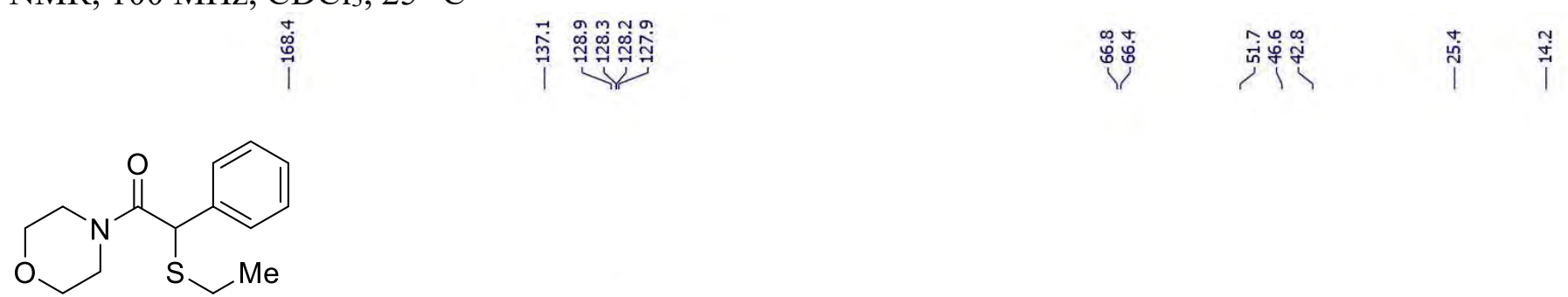

$4 p$

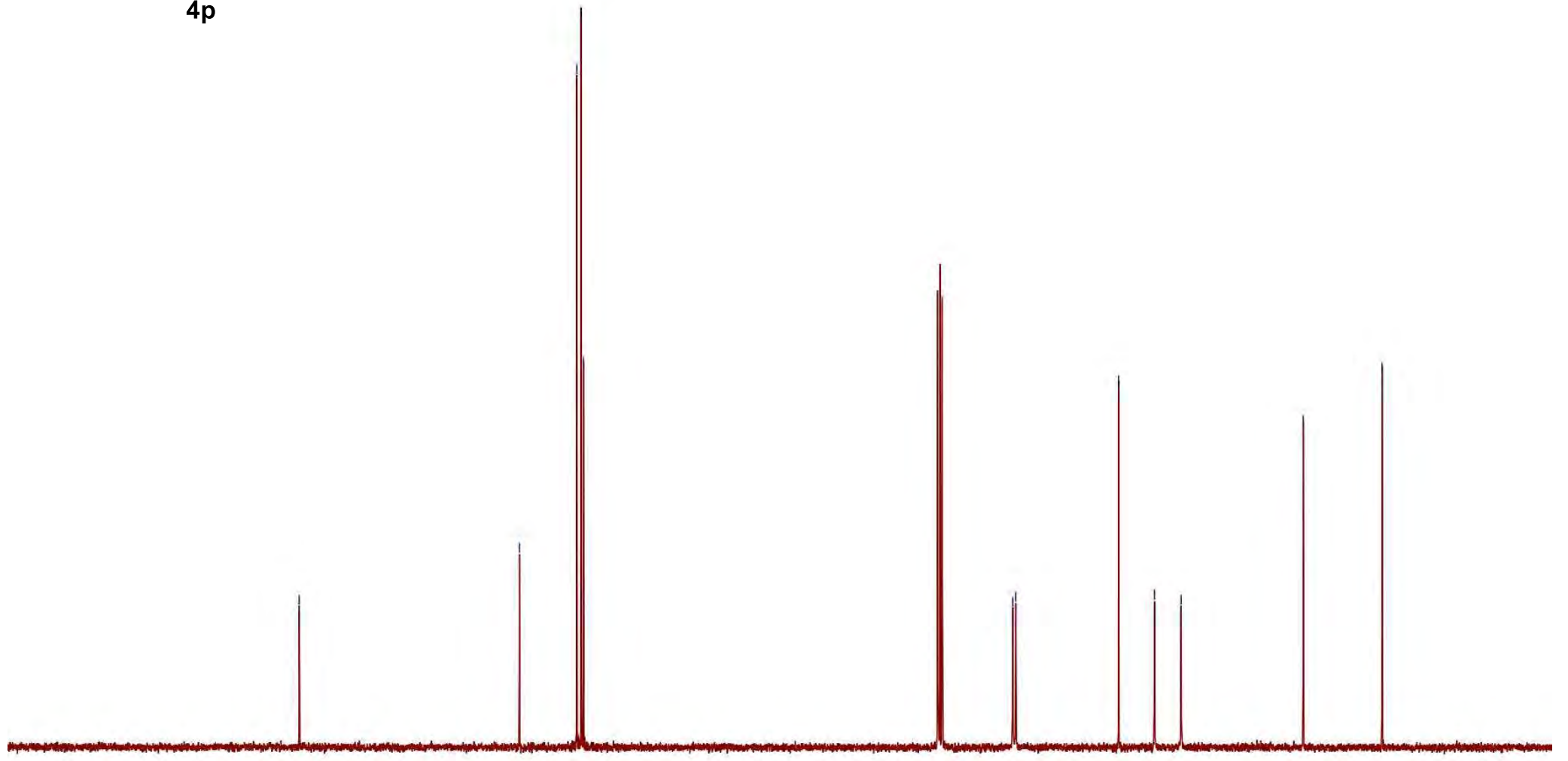

$.0 \quad 200 \quad 190$ 
${ }^{1} \mathrm{H} \mathrm{NMR}, 400 \mathrm{MHz}, \mathrm{CDCl}_{3}, 25^{\circ} \mathrm{C}$
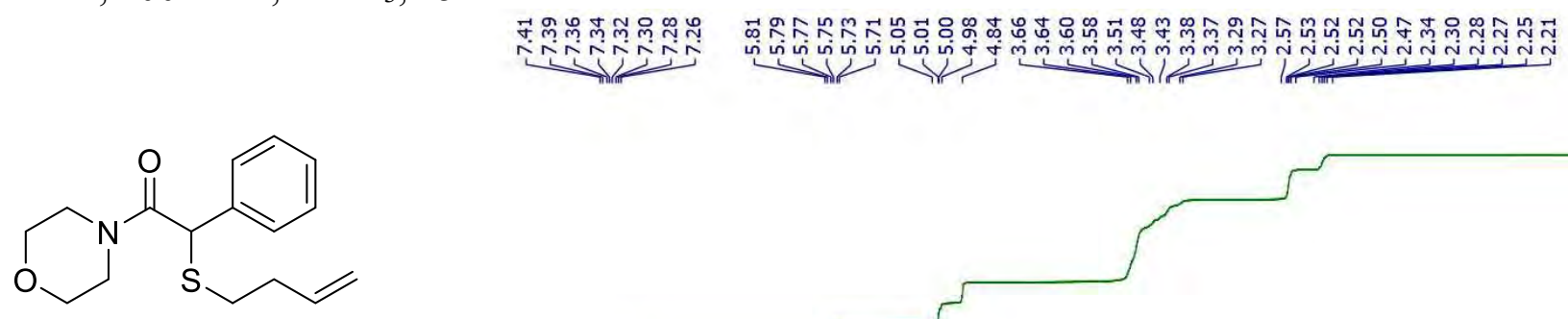

$4 q$

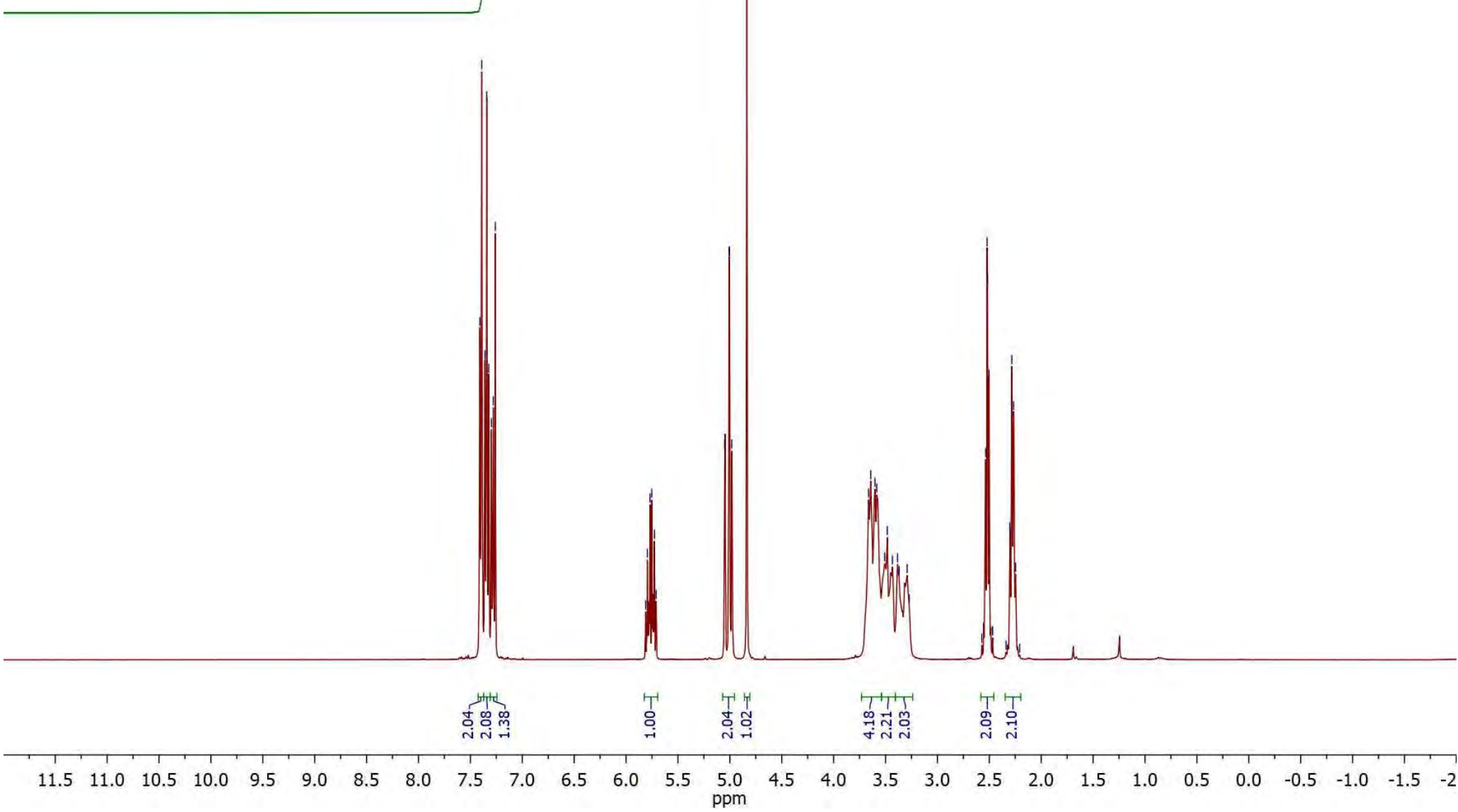


${ }^{13} \mathrm{C}$ NMR, $100 \mathrm{MHz}, \mathrm{CDCl}_{3}, 25{ }^{\circ} \mathrm{C}$

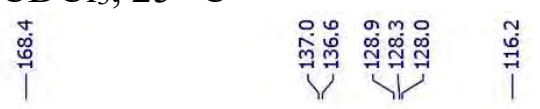

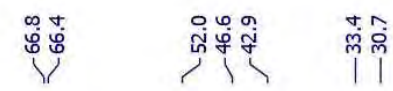

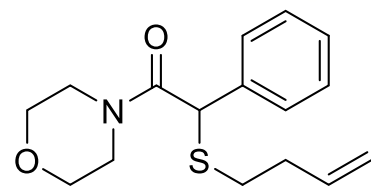

$4 q$

$4 q$

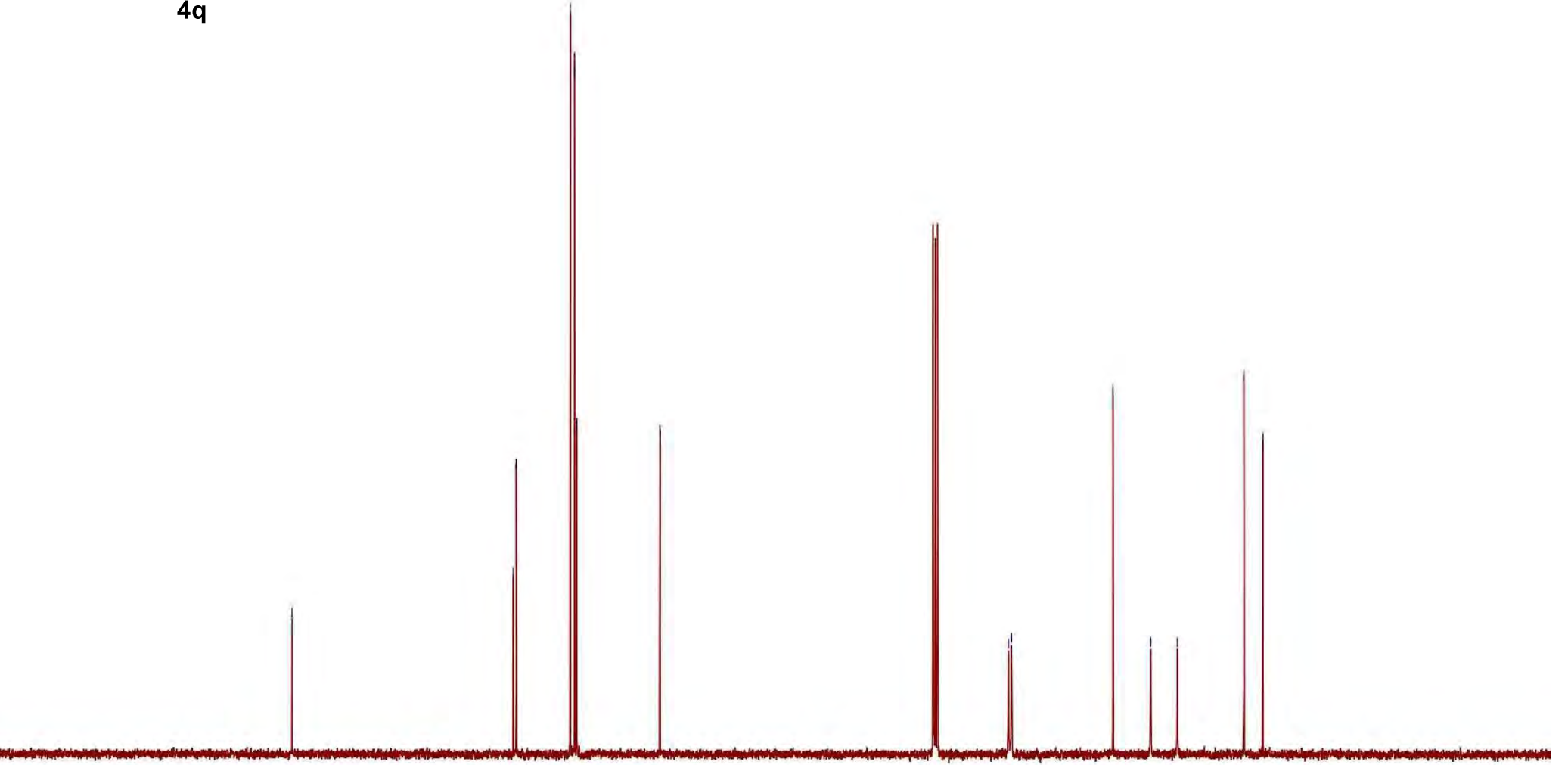

$10 \quad 200 \quad 190$

180

160

150140

130

$120 \quad 110$ 
${ }^{1} \mathrm{H} \mathrm{NMR}, 400 \mathrm{MHz}, \mathrm{CDCl}_{3}, 25^{\circ} \mathrm{C}$
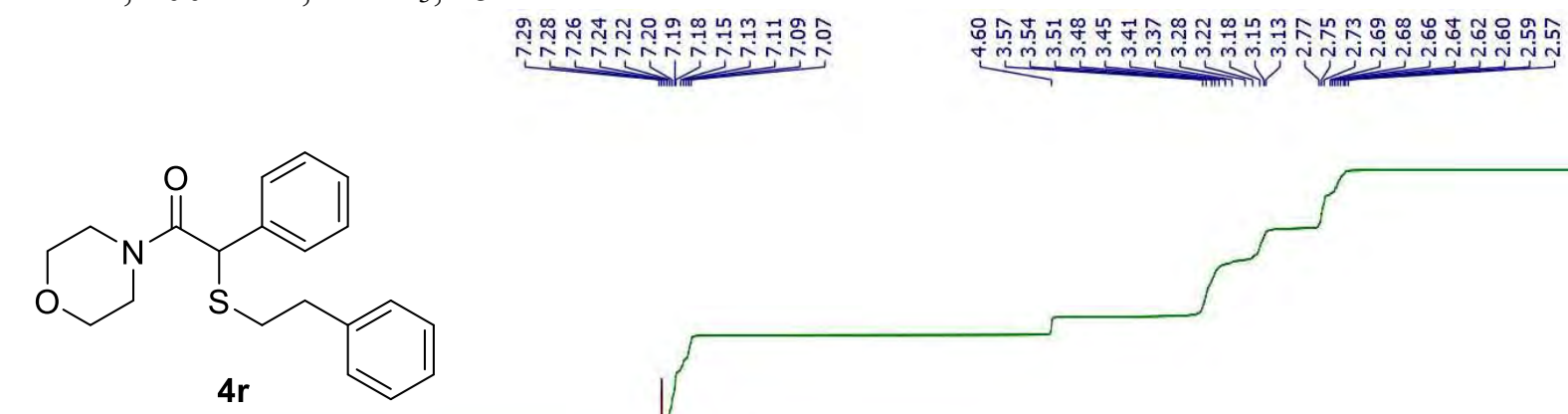

0
0

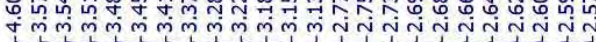

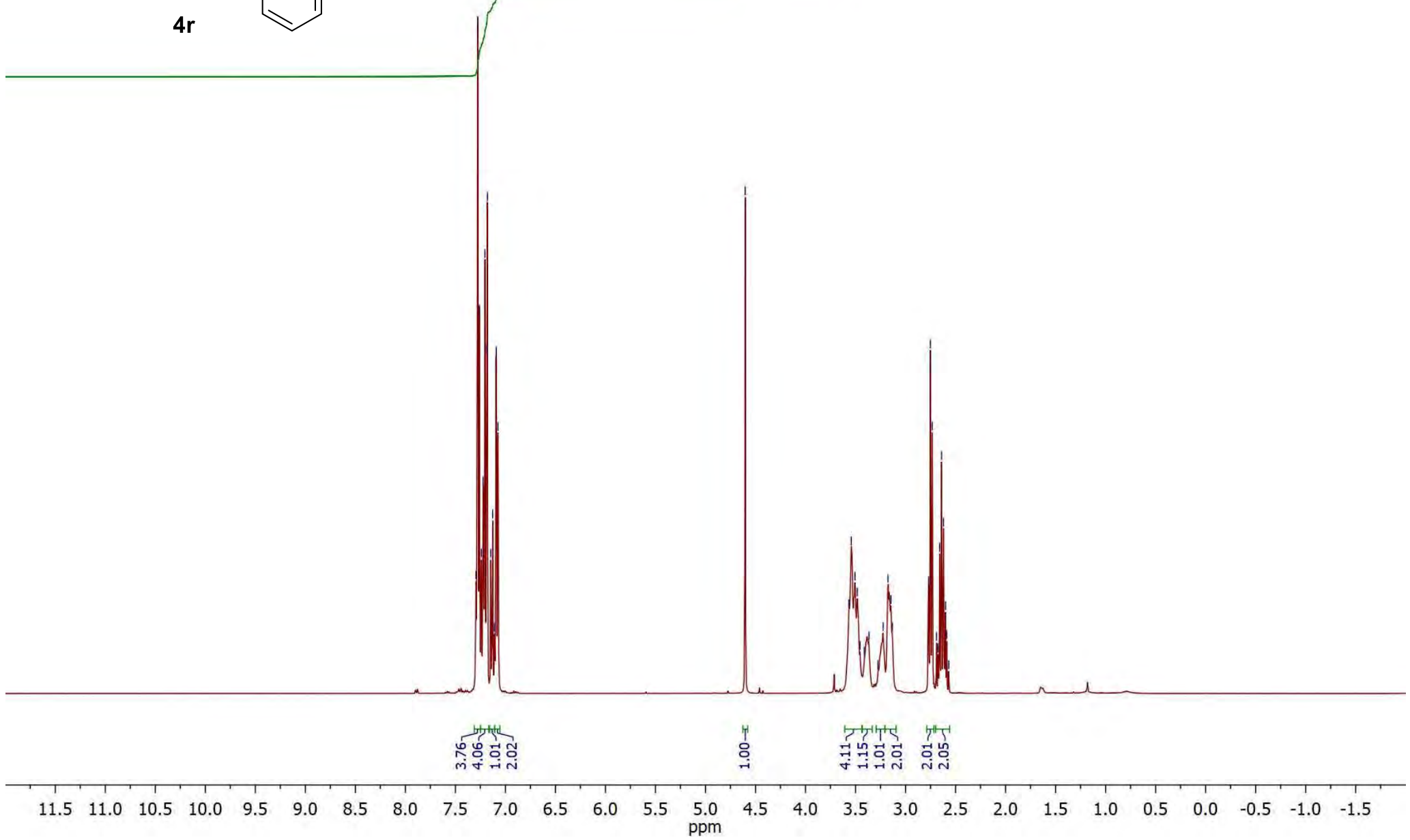


${ }^{13} \mathrm{C} \mathrm{NMR}, 100 \mathrm{MHz}, \mathrm{CDCl}_{3}, 25{ }^{\circ} \mathrm{C}$
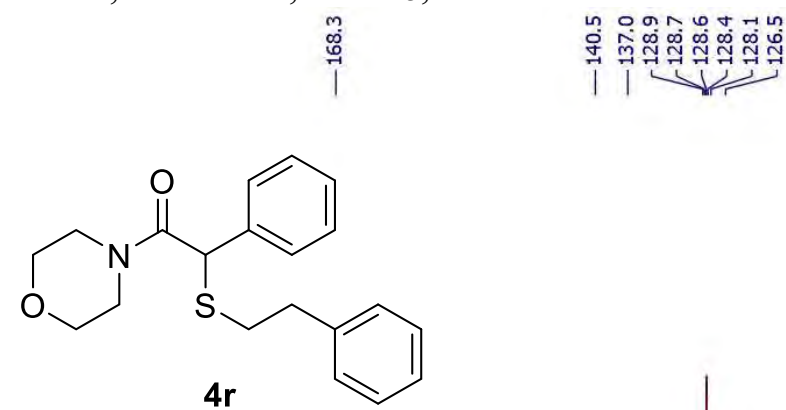

$4 r$

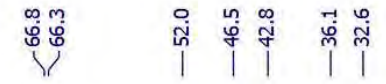

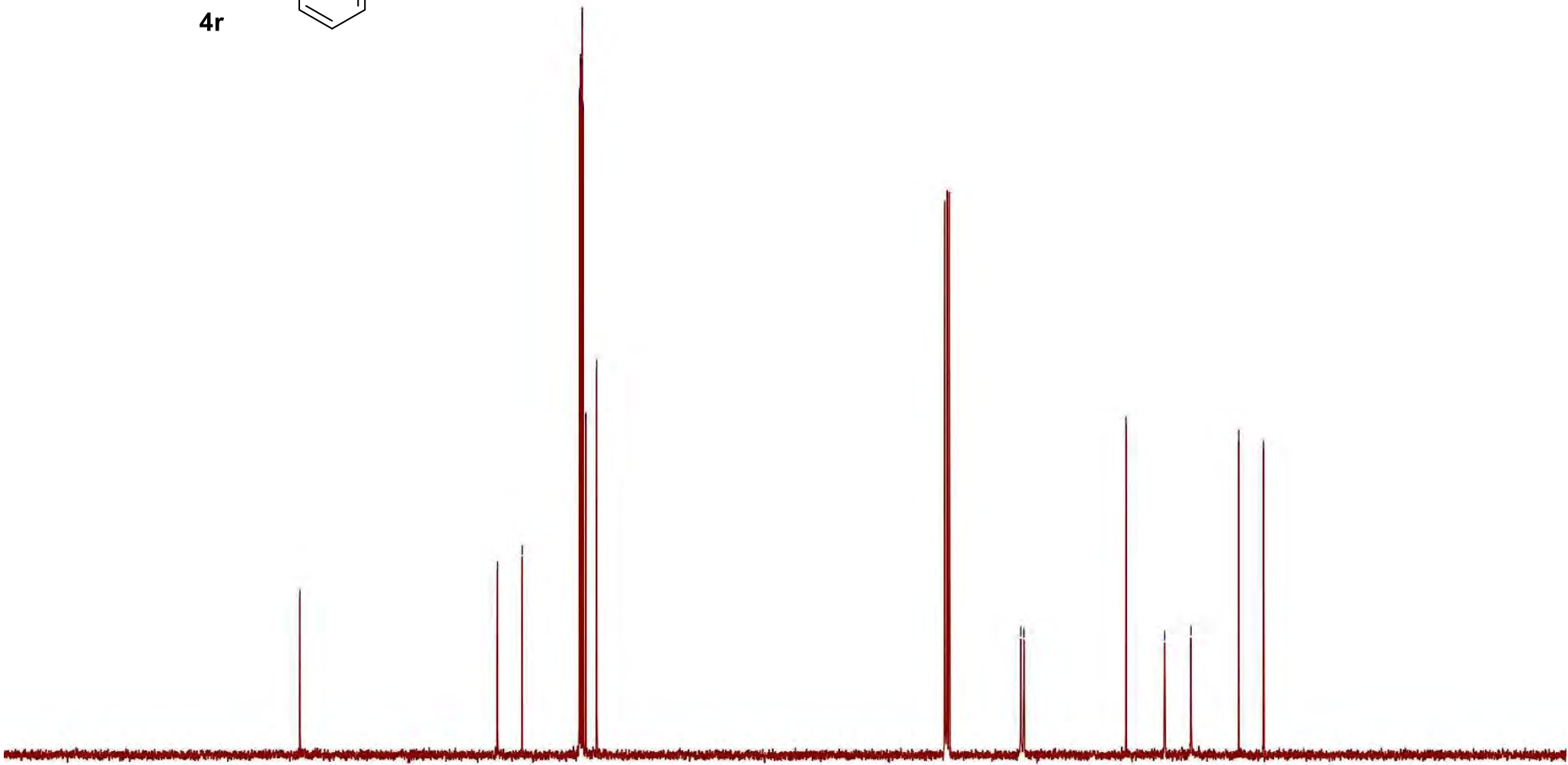

$.0 \quad 200 \quad 190$ 
${ }^{1} \mathrm{H} \mathrm{NMR}, 400 \mathrm{MHz}, \mathrm{CDCl}_{3}, 25^{\circ} \mathrm{C}$
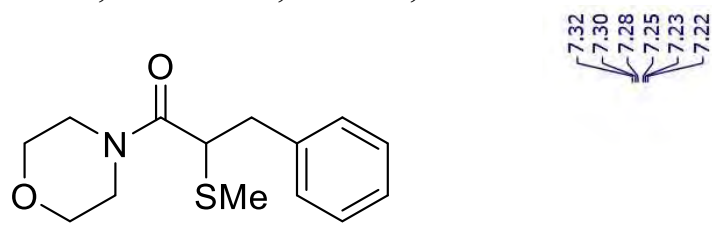

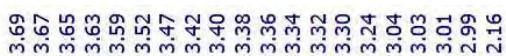

4s
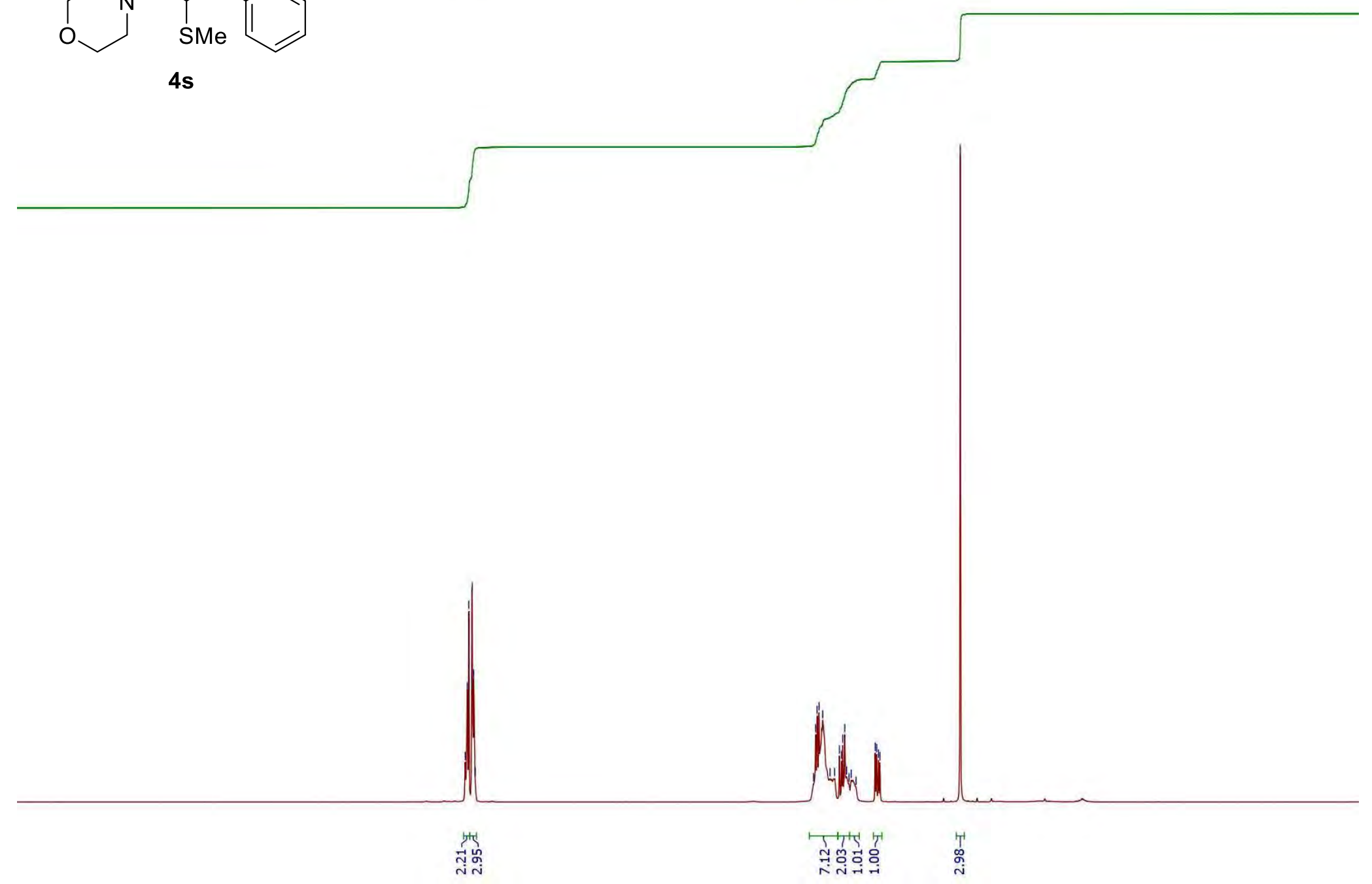

$\begin{array}{lllllllllllllllllllllllllllllllll}11.5 & 11.0 & 10.5 & 10.0 & 9.5 & 9.0 & 8.5 & 8.0 & 7.5 & 7.0 & 6.5 & 6.0 & 5.5 & \begin{array}{c}5.0 \\ \mathrm{ppm}\end{array} & 4.5 & 4.0 & 3.5 & 3.0 & 2.5 & 2.0 & 1.5 & 1.0 & 0.5 & 0.0 & -0.5 & -1.0 & -1.5 & -2\end{array}$ 
${ }^{13} \mathrm{C} \mathrm{NMR}, 100 \mathrm{MHz}, \mathrm{CDCl}_{3}, 25{ }^{\circ} \mathrm{C}$
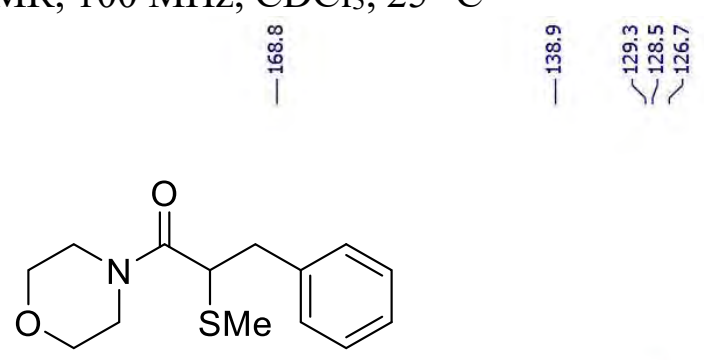

4s

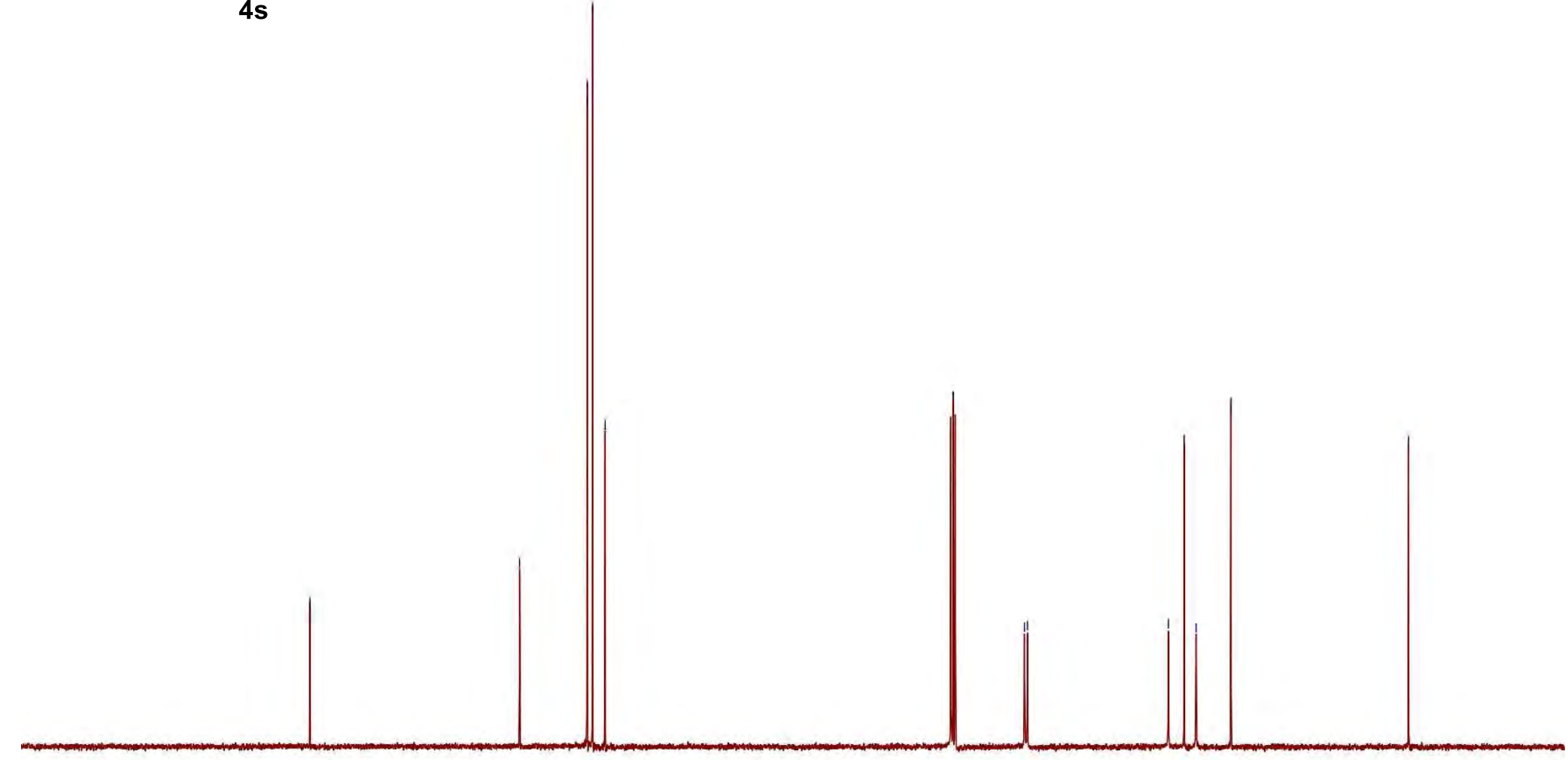

$0 \quad 200 \quad 190$ 
${ }^{1} \mathrm{H} \mathrm{NMR}, 400 \mathrm{MHz}, \mathrm{CDCl}_{3}, 25^{\circ} \mathrm{C}$

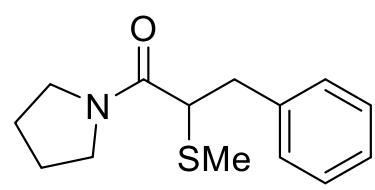

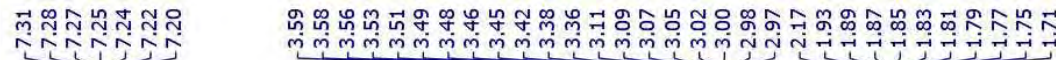

4t
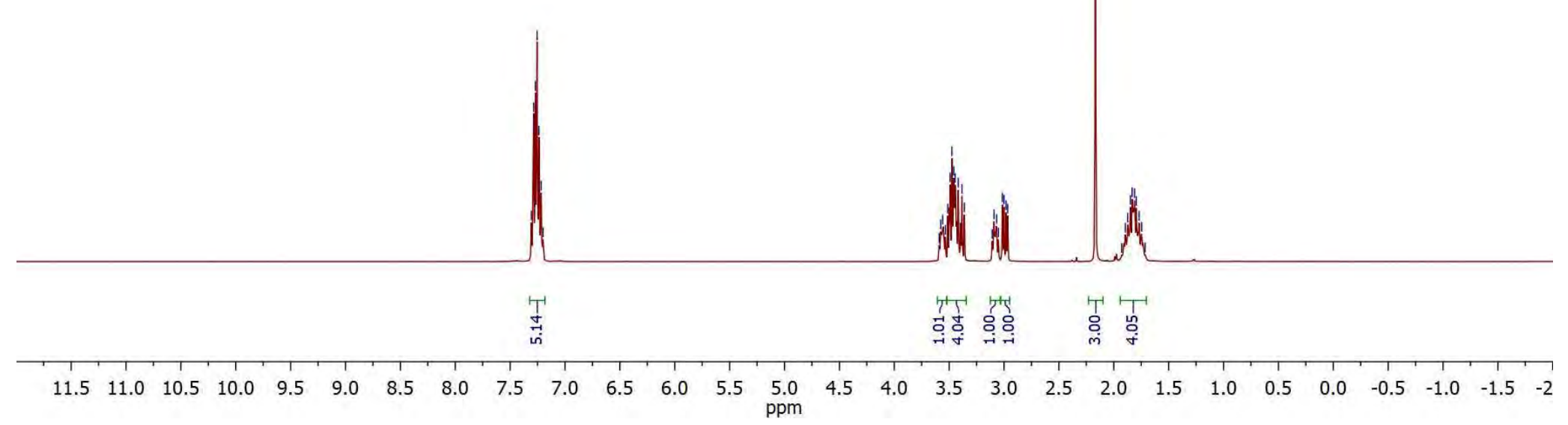
Chemoselective $\alpha$-Sulfidation of Amides Using Sulfoxide Reagents

M. Leypold, K. A. D’Angelo and M. Movassaghi

Page S163 / S183

${ }^{13} \mathrm{C} \mathrm{NMR,} 100 \mathrm{MHz}, \mathrm{CDCl}_{3}, 25{ }^{\circ} \mathrm{C}$

ปั

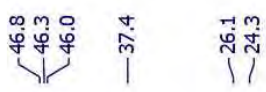

$\stackrel{\text { กี }}{7}$<smiles>CSC(Cc1ccccc1)C(=O)N1CCCC1</smiles>

$4 t$
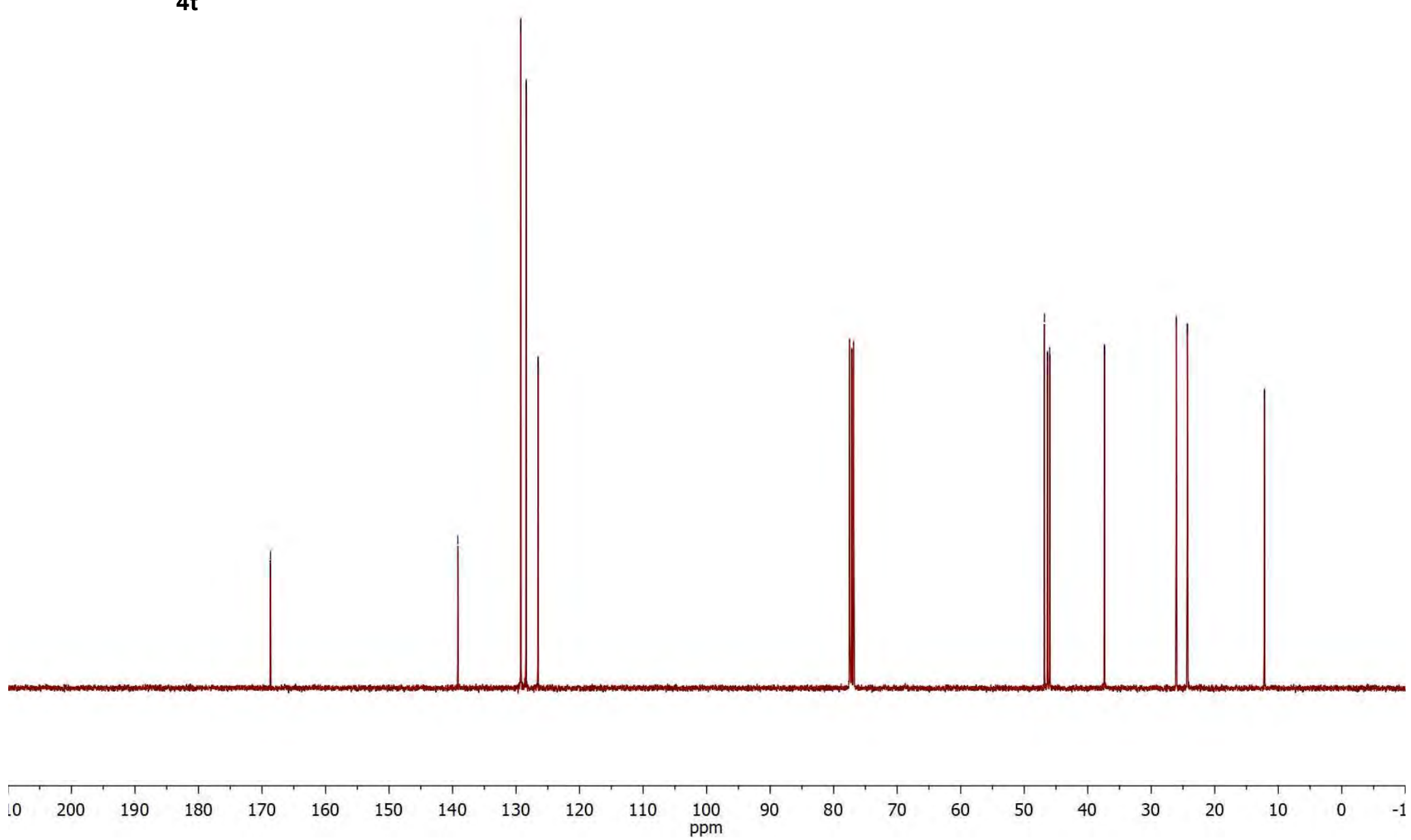
${ }^{1} \mathrm{H} \mathrm{NMR}, 400 \mathrm{MHz}, \mathrm{CDCl}_{3}, 25^{\circ} \mathrm{C}$

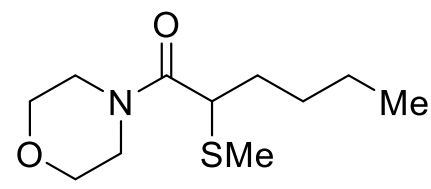

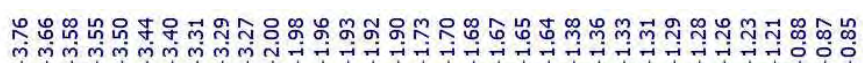

$4 u$

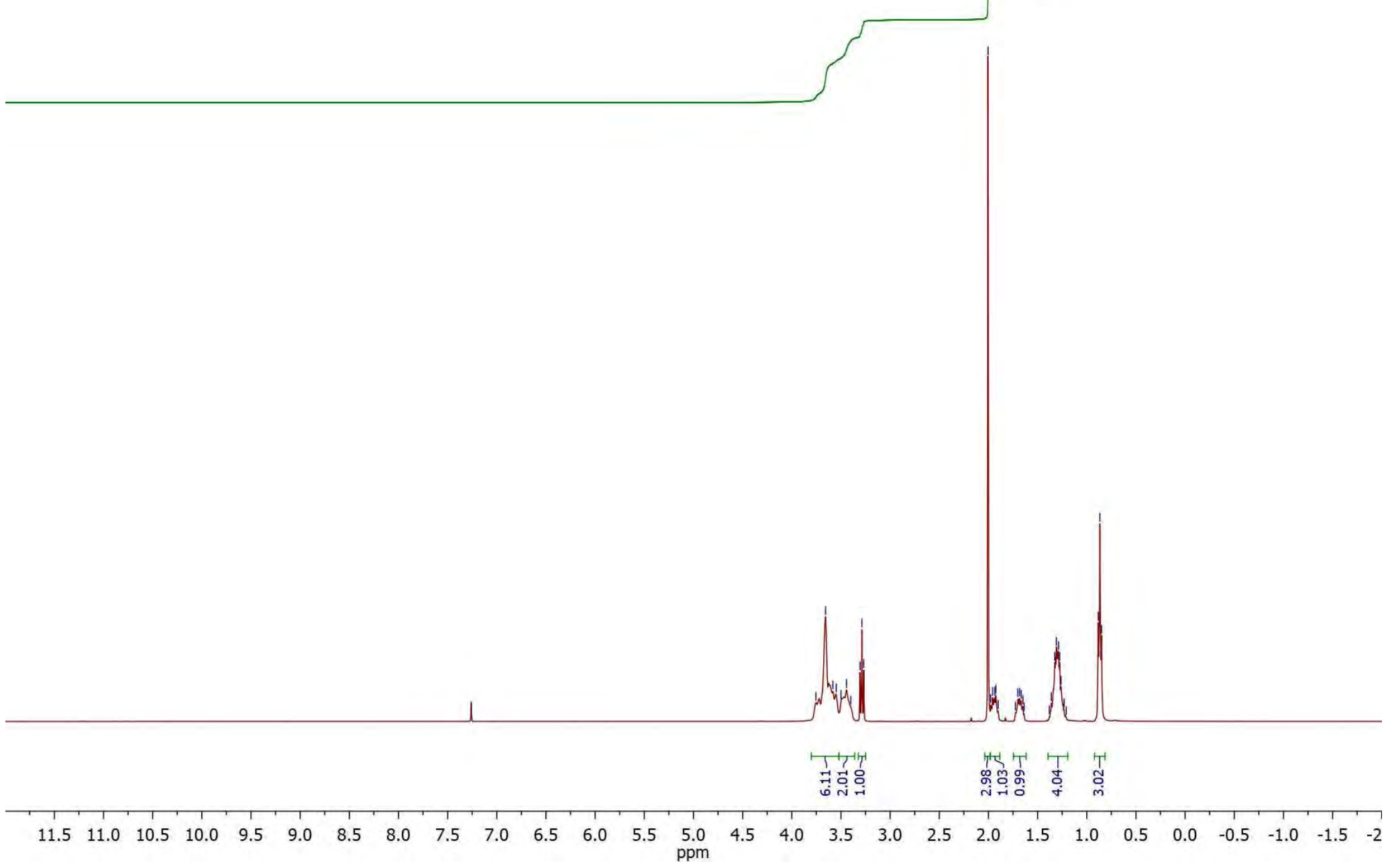


${ }^{13} \mathrm{C} \mathrm{NMR}, 100 \mathrm{MHz}, \mathrm{CDCl}_{3}, 25{ }^{\circ} \mathrm{C}$

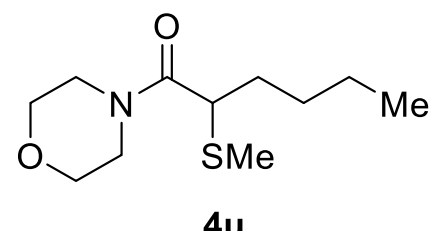

$4 u$

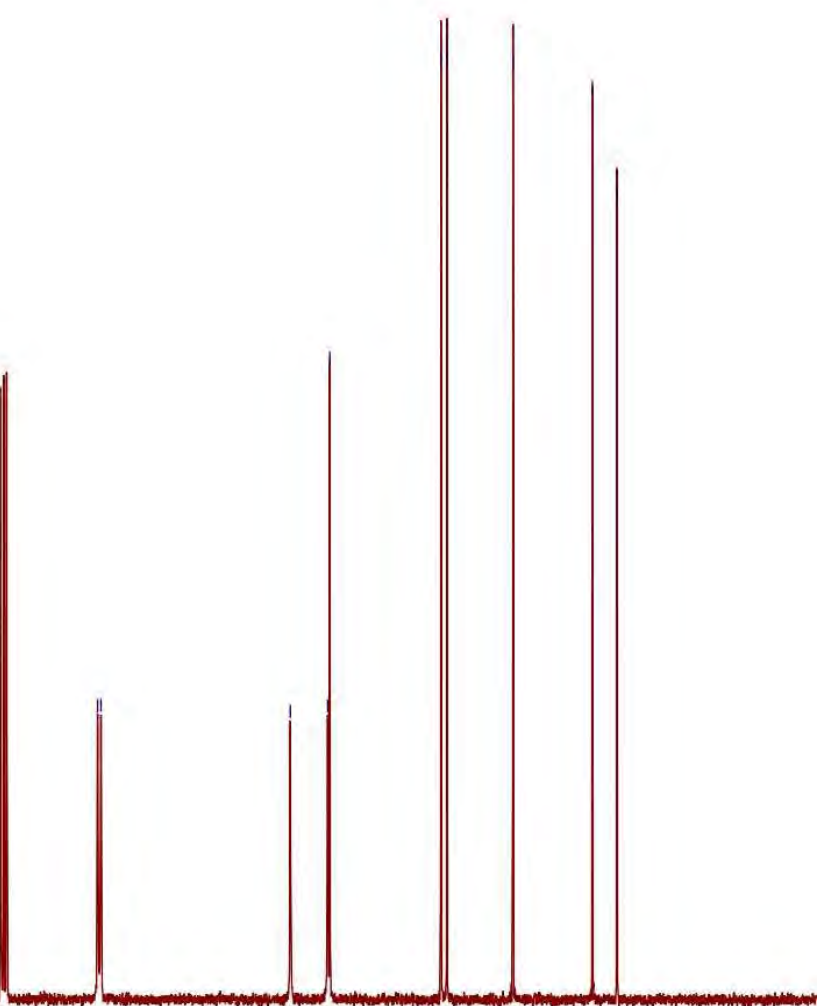

$0 \quad 200-190$ 
${ }^{1} \mathrm{H} \mathrm{NMR}, 400 \mathrm{MHz}, \mathrm{CDCl}_{3}, 25^{\circ} \mathrm{C}$

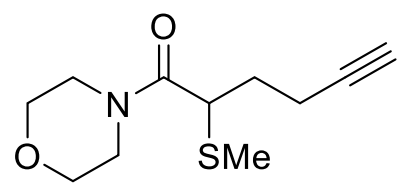

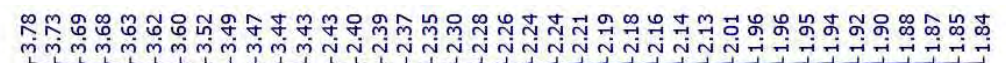

$4 v$
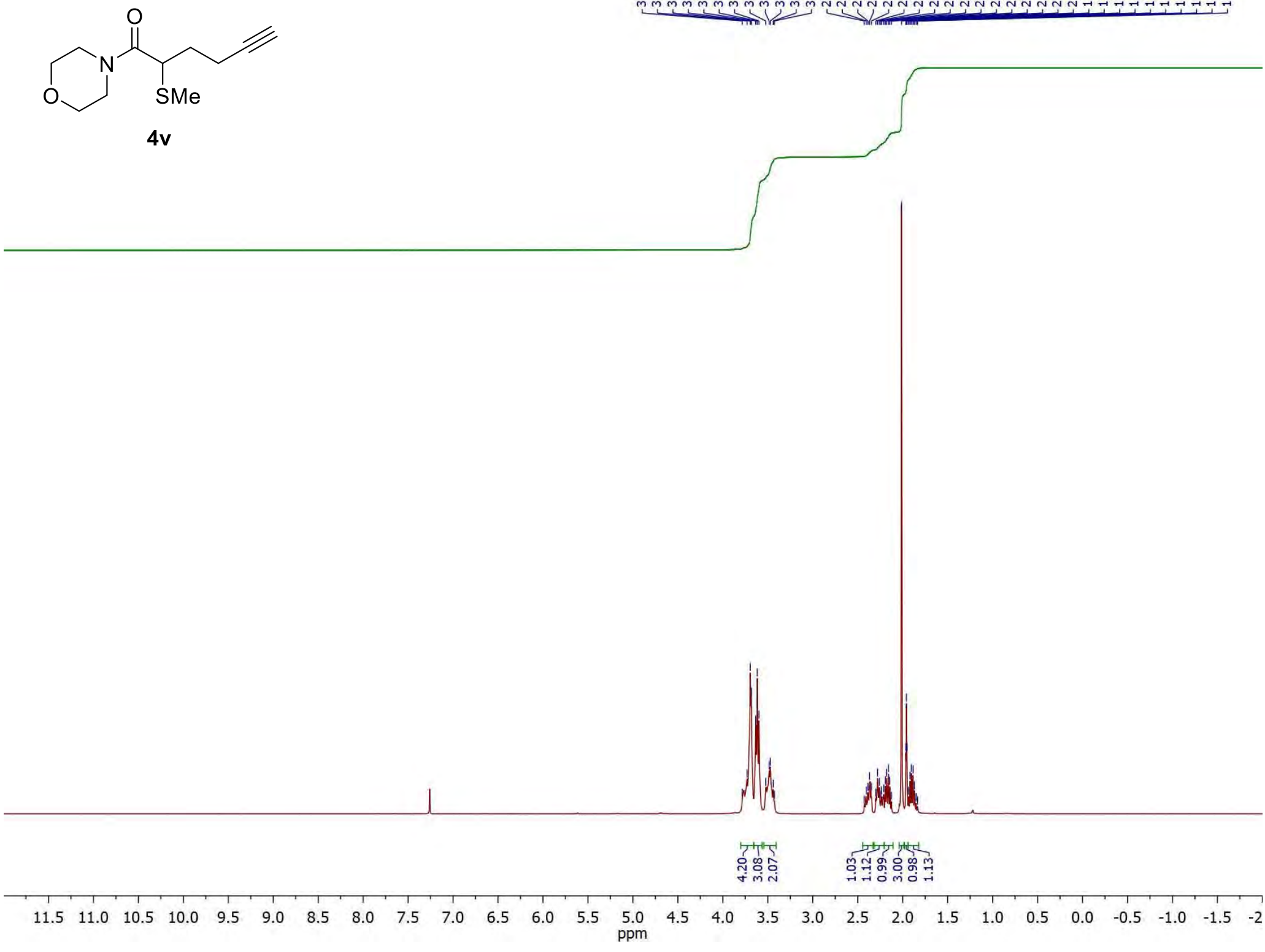
${ }^{13} \mathrm{C}$ NMR, $100 \mathrm{MHz}, \mathrm{CDCl}_{3}, 25^{\circ} \mathrm{C}$<smiles>C#CCCC(C)C(=O)N1CCOCC1</smiles>

$4 \mathrm{v}$

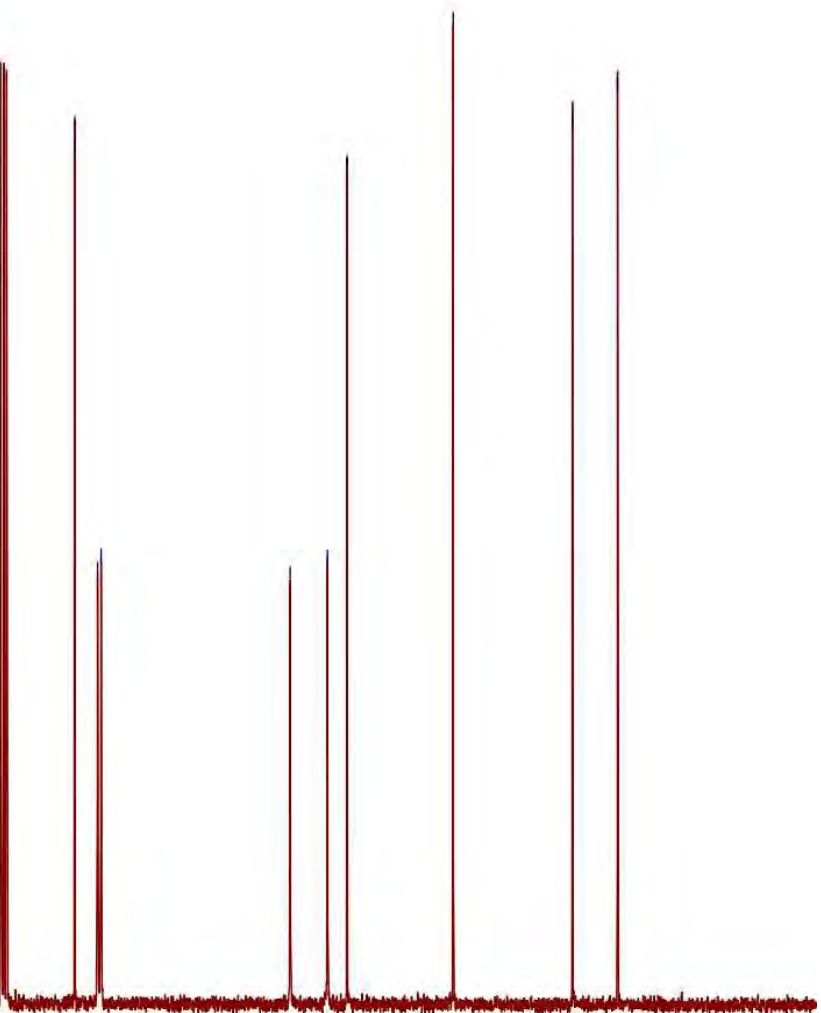

$0 \quad 200 \quad 190$ 
${ }^{1} \mathrm{H} \mathrm{NMR}, 400 \mathrm{MHz}, \mathrm{CDCl}_{3}, 25^{\circ} \mathrm{C}$

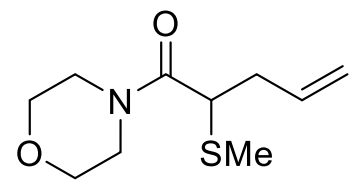

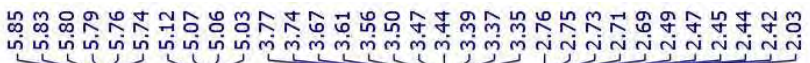

$4 w$

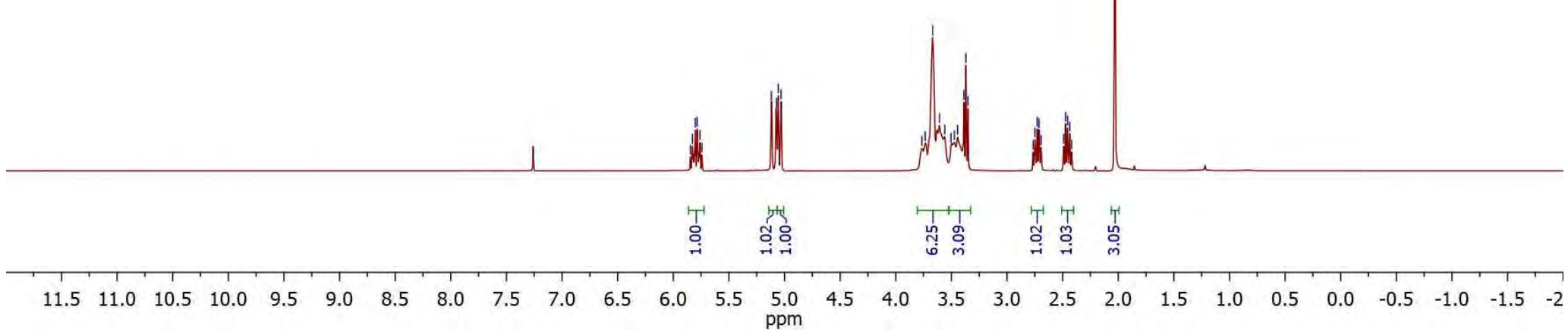


Chemoselective $\alpha$-Sulfidation of Amides Using Sulfoxide Reagents

M. Leypold, K. A. D’Angelo and M. Movassaghi

Page S169 / S183

${ }^{13} \mathrm{C} \mathrm{NMR,} 100 \mathrm{MHz}, \mathrm{CDCl}_{3}, 25^{\circ} \mathrm{C}$

$\stackrel{\substack{n \\ 0}}{\substack{\infty \\ \stackrel{1}{1}}}$

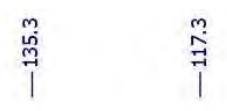

苞

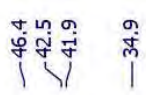

$\stackrel{\text { में }}{\overrightarrow{1}}$<smiles>C=CCC(C)C(=O)N1CCOCC1</smiles>

4w
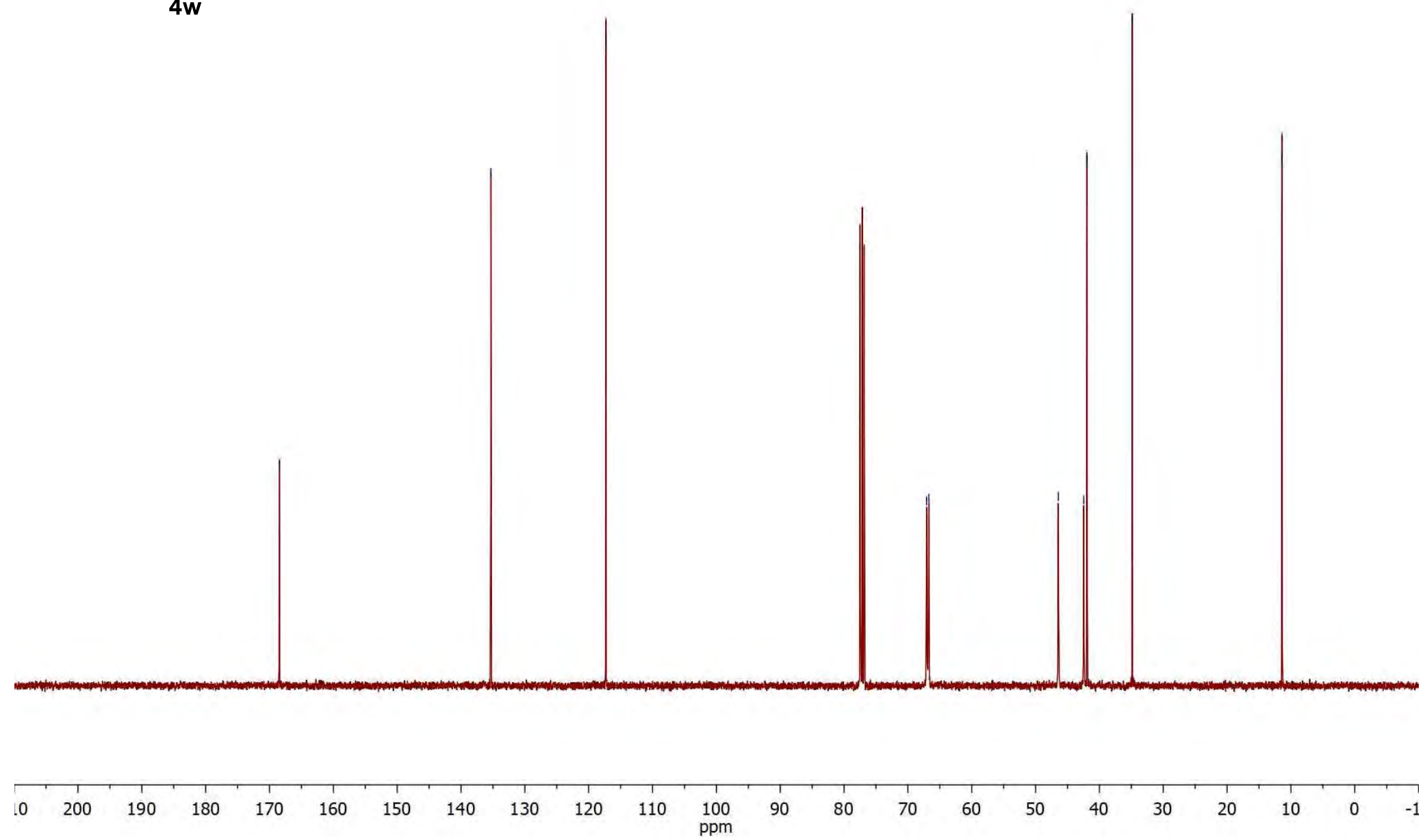
${ }^{1} \mathrm{H} \mathrm{NMR}, 400 \mathrm{MHz}, \mathrm{CDCl}_{3}, 25^{\circ} \mathrm{C}$

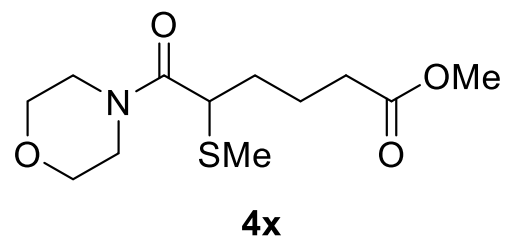

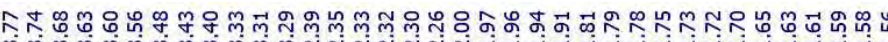

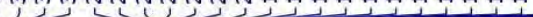

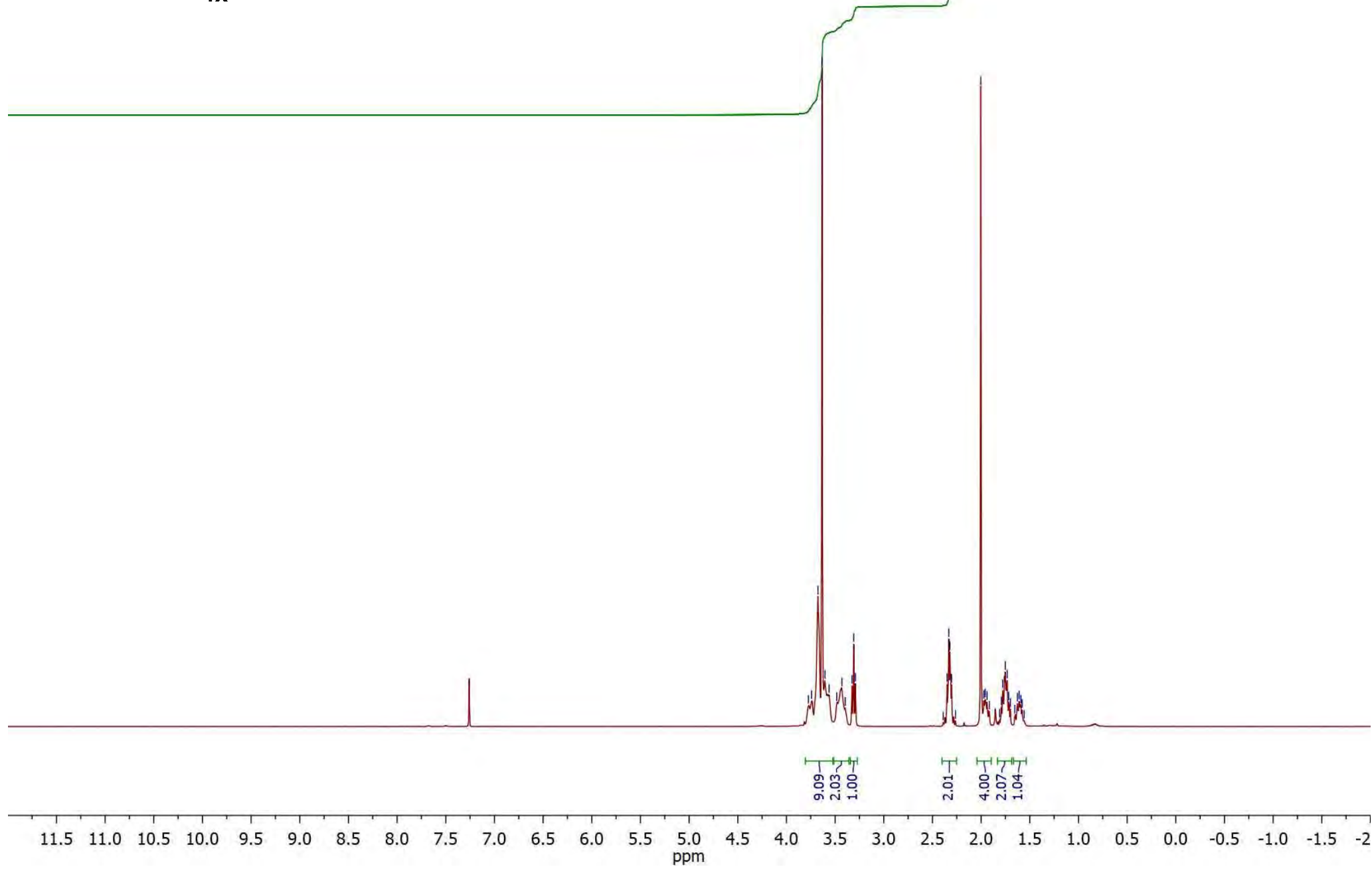


${ }^{13} \mathrm{C} \mathrm{NMR,} 100 \mathrm{MHz}, \mathrm{CDCl}_{3}, 25^{\circ} \mathrm{C}$

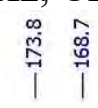<smiles>COC(=O)CCCC([As])C(=O)N1CCOCC1</smiles>

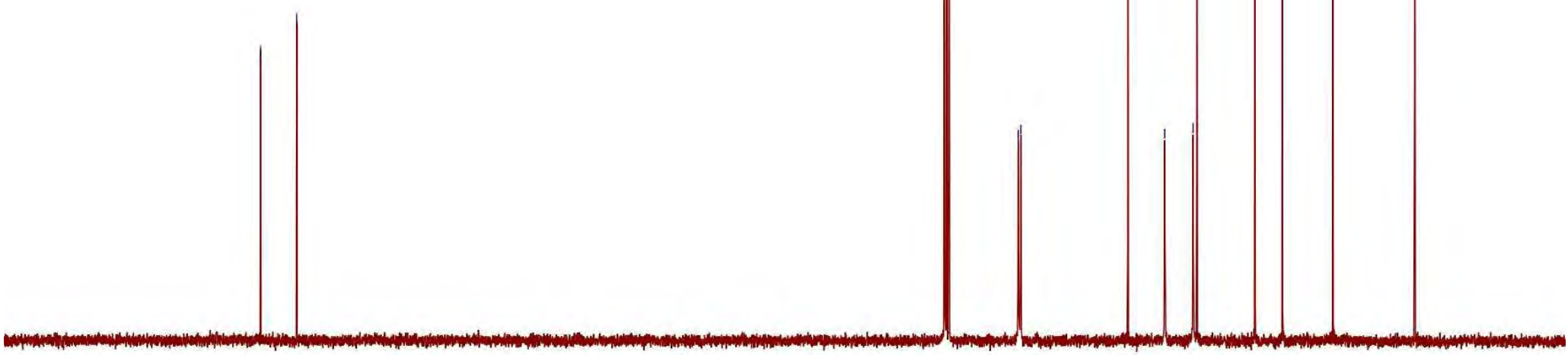

$0 \quad 200 \quad 190-180$

160 
${ }^{1} \mathrm{H} \mathrm{NMR}, 400 \mathrm{MHz}, \mathrm{CDCl}_{3}, 25^{\circ} \mathrm{C}$
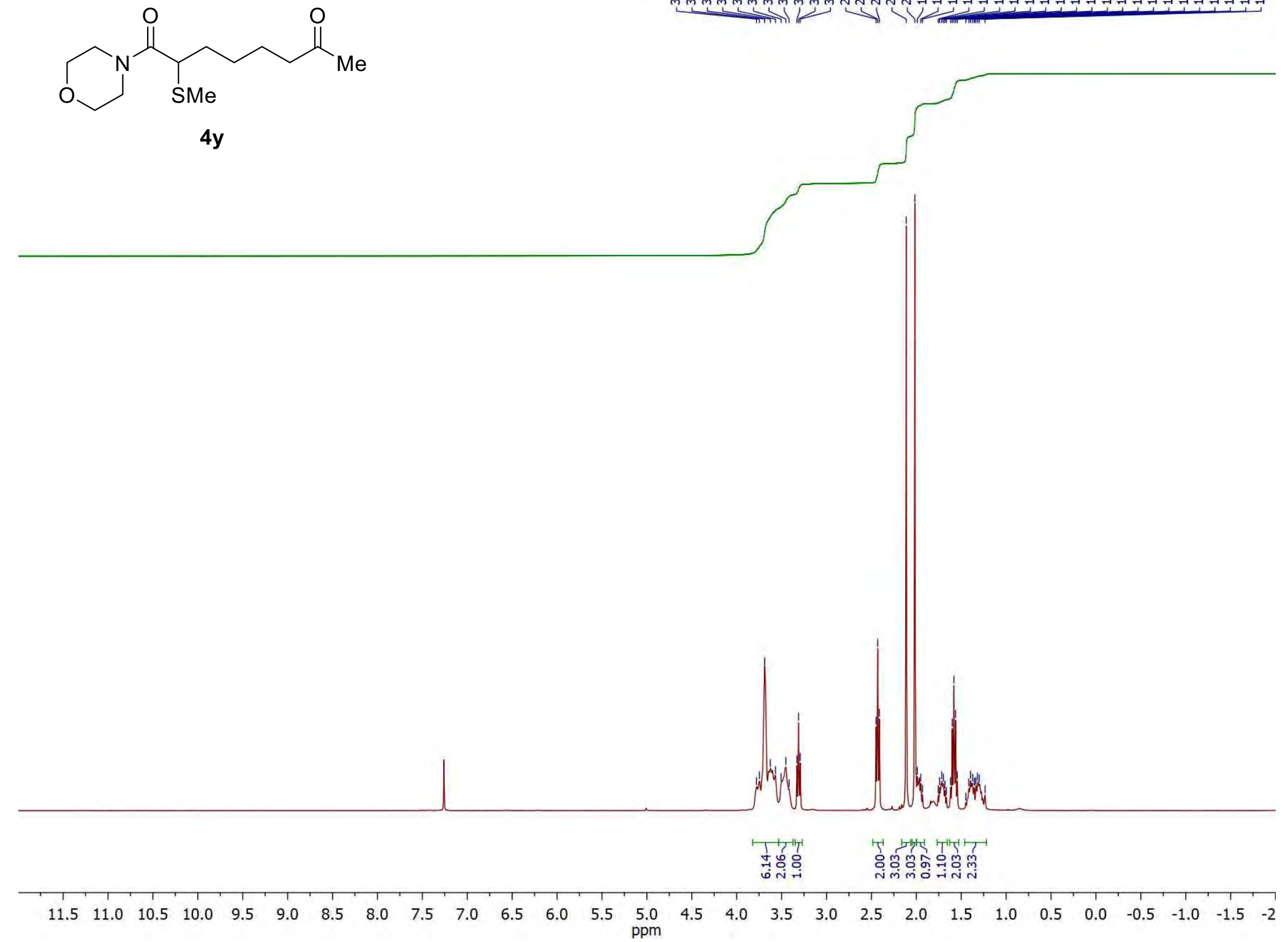
${ }^{13} \mathrm{C} \mathrm{NMR}, 100 \mathrm{MHz}, \mathrm{CDCl}_{3}, 25^{\circ} \mathrm{C}$<smiles>COCCCCCCCCCCCO</smiles><smiles>CC(=O)CCCCC(C(=O)N1CCOCC1)S(C)(C)C</smiles>

4y

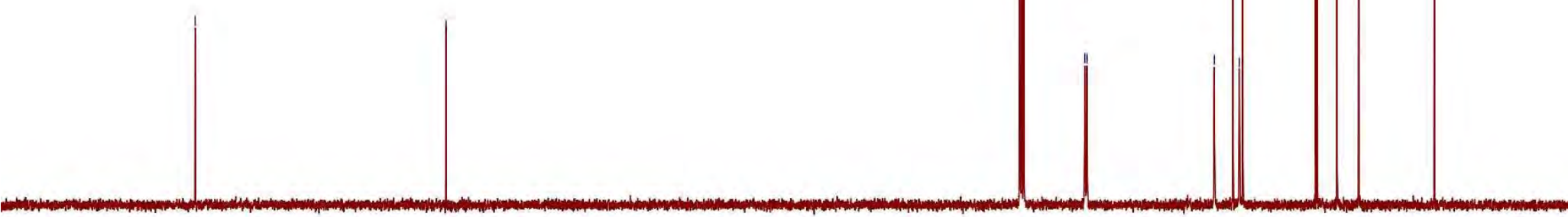

$\begin{array}{llllllllllllllllllllllllll} & 230 & 220 & 210 & 200 & 190 & 180 & 170 & 160 & 150 & 140 & 130 & 120 & 110 & 100 & 90 & 80 & 70 & 60 & 50 & 40 & 30 & 20 & 10 & 0 & -1\end{array}$ 
${ }^{1} \mathrm{H} \mathrm{NMR}, 400 \mathrm{MHz}, \mathrm{CDCl}_{3}, 25^{\circ} \mathrm{C}$
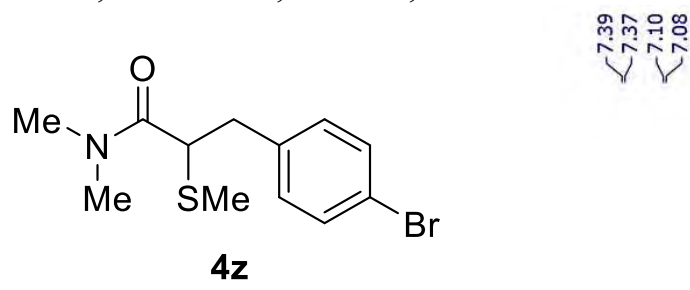

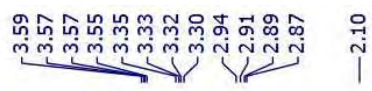

$$
\mathrm{Br}
$$
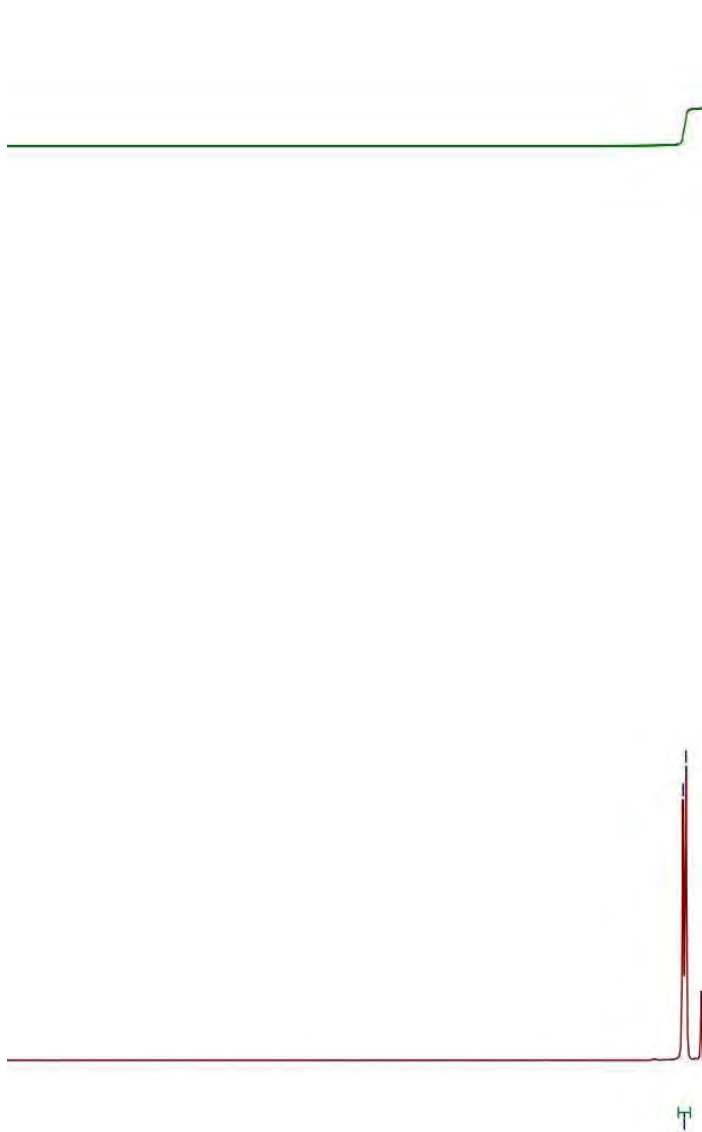

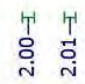

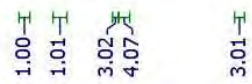

$\begin{array}{llllllllllllllllllllllllllllllllll}11.5 & 11.0 & 10.5 & 10.0 & 9.5 & 9.0 & 8.5 & 8.0 & 7.5 & 7.0 & 6.5 & 6.0 & 5.5 & \begin{array}{c}5.0 \\ \mathrm{ppm}\end{array} & 4.5 & 4.0 & 3.5 & 3.0 & 2.5 & 2.0 & 1.5 & 1.0 & 0.5 & 0.0 & -0.5 & -1.0 & -1.5 & -2\end{array}$ 
${ }^{13} \mathrm{C} \mathrm{NMR}, 100 \mathrm{MHz}, \mathrm{CDCl}_{3}, 25{ }^{\circ} \mathrm{C}$
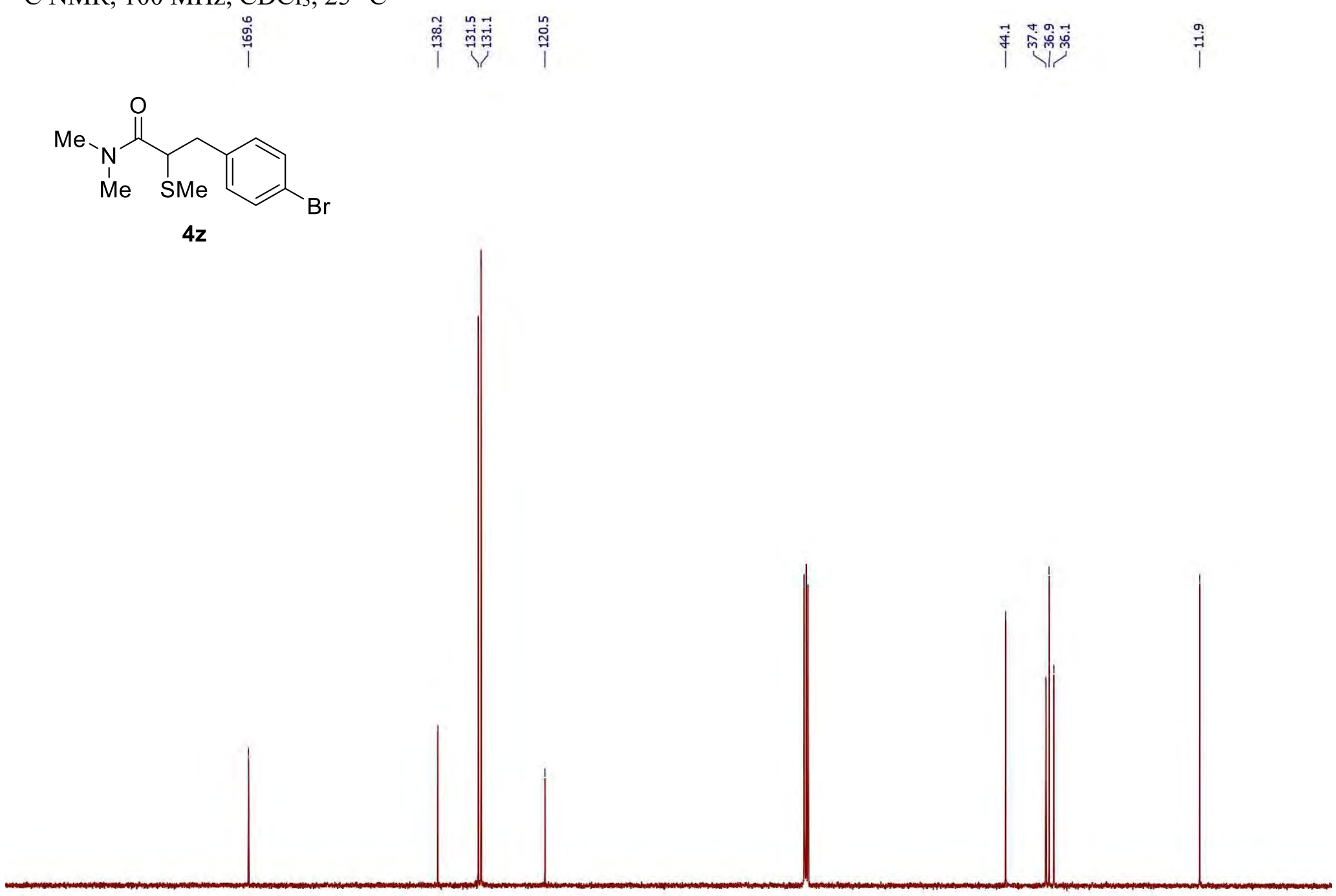

$\begin{array}{llll}0 & 200 & 190 & 180\end{array}$ $-1$ 
${ }^{1} \mathrm{H} \mathrm{NMR}, 400 \mathrm{MHz}, \mathrm{CDCl}_{3}, 25^{\circ} \mathrm{C}$
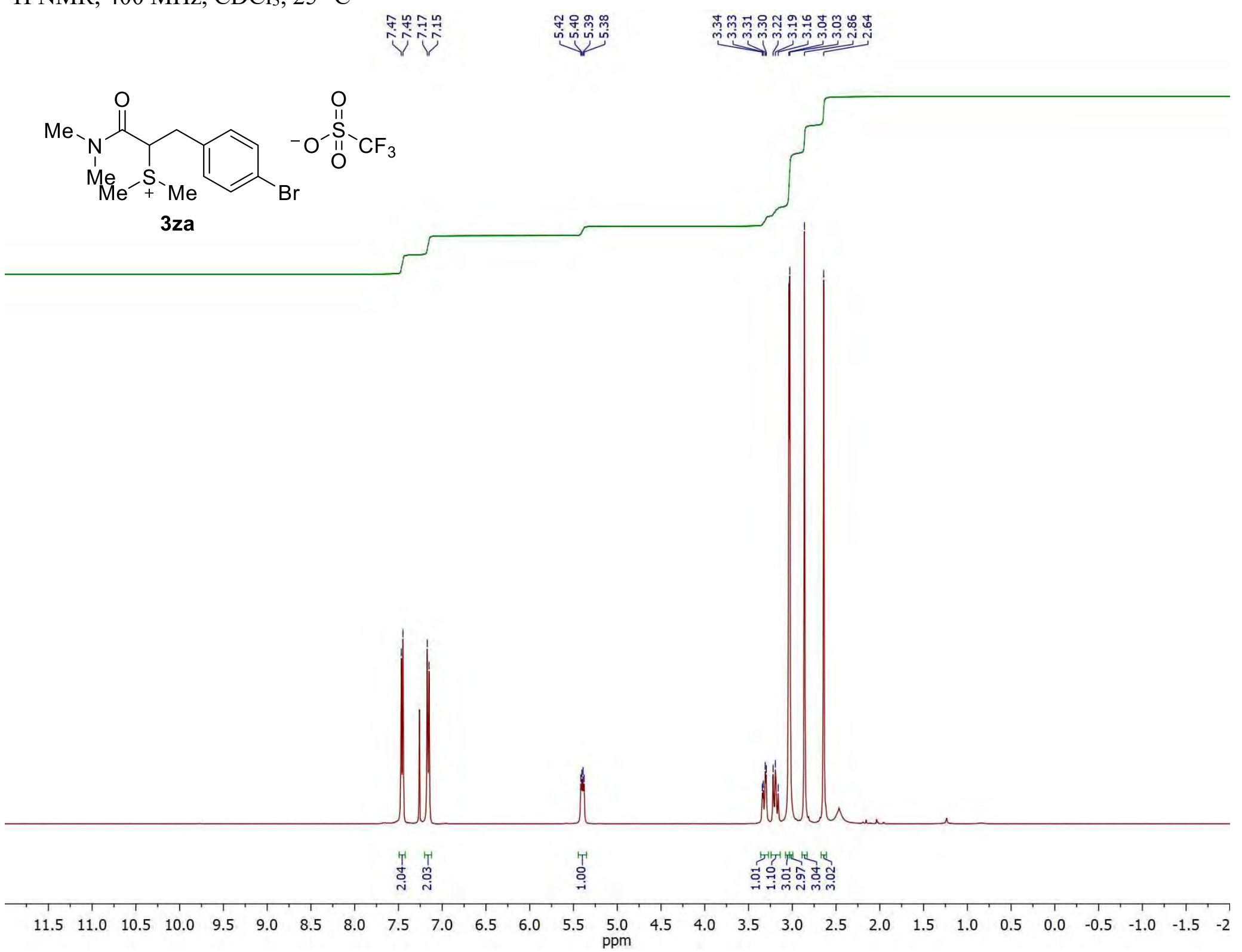
${ }^{13} \mathrm{C} \mathrm{NMR}, 100 \mathrm{MHz}, \mathrm{CDCl}_{3}, 25{ }^{\circ} \mathrm{C}$
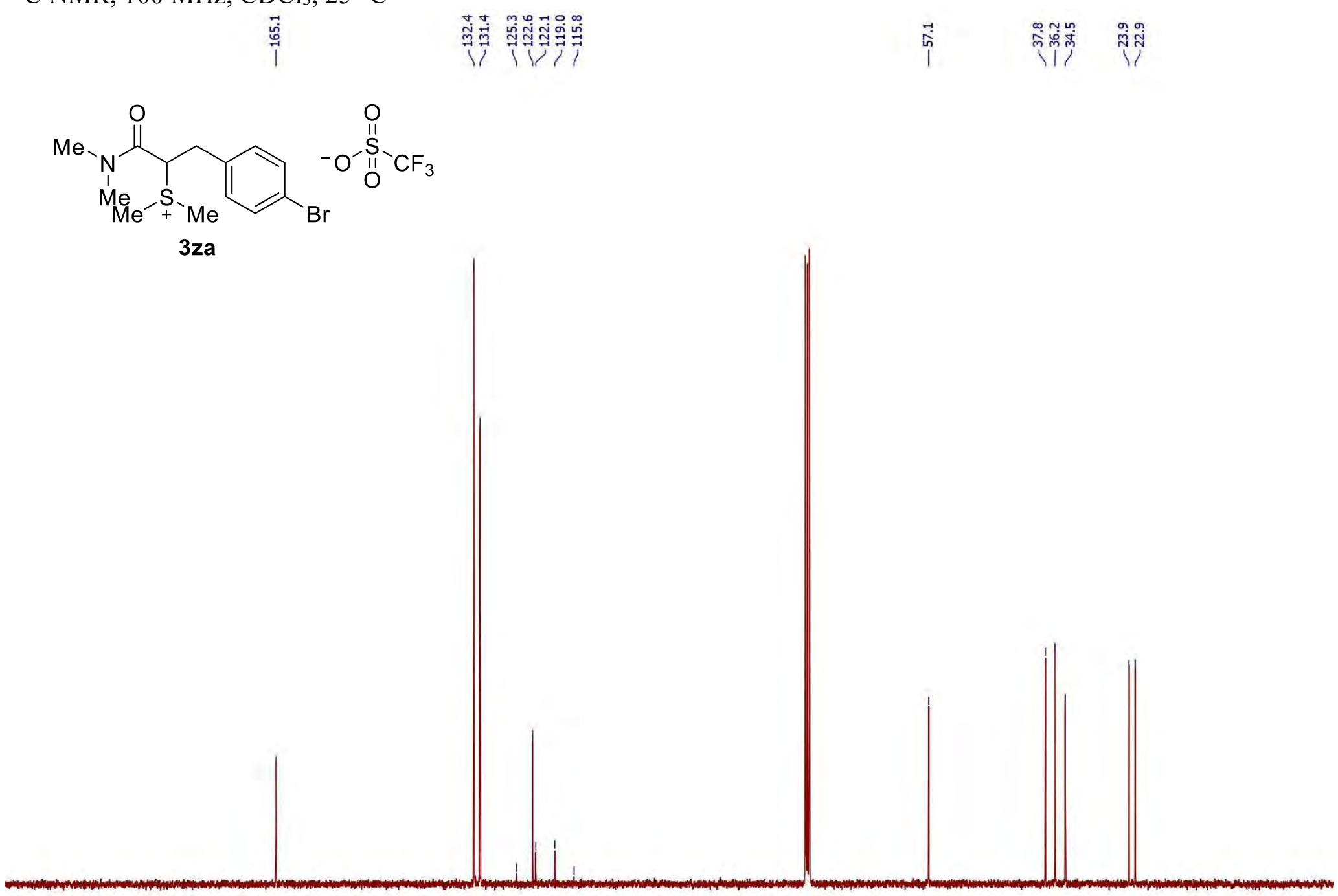

$0 \quad 200 \quad 190$ 
${ }^{19} \mathrm{~F} \mathrm{NMR}, 376 \mathrm{MHz}, \mathrm{CDCl}_{3}, 25{ }^{\circ} \mathrm{C}$
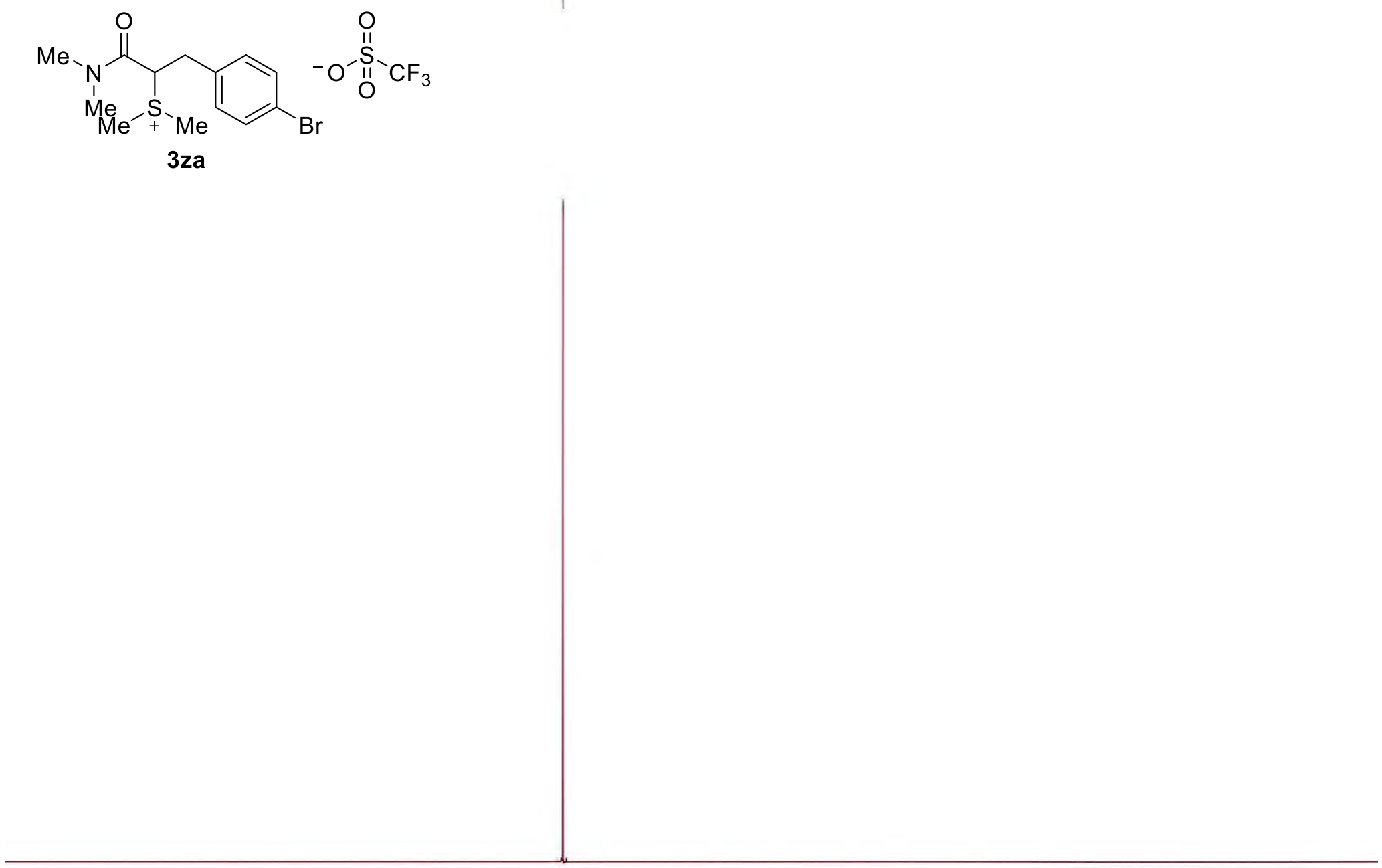

\begin{tabular}{llllllllllllllllllllll}
\hline 0 & -10 & -20 & -30 & -40 & -50 & -60 & -70 & -80 & -90 & -100 & -110 & -120 & -130 & -140 & -150 & -160 & -170 & -180 & -190 & -200 & -2
\end{tabular}


${ }^{1} \mathrm{H} \mathrm{NMR}, 400 \mathrm{MHz}, \mathrm{CDCl}_{3}, 25{ }^{\circ} \mathrm{C}$

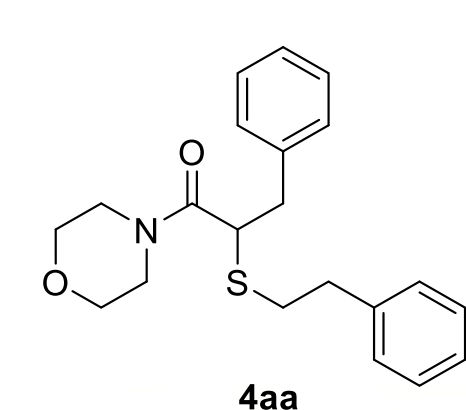
ํำ

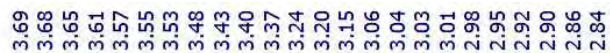

4aa

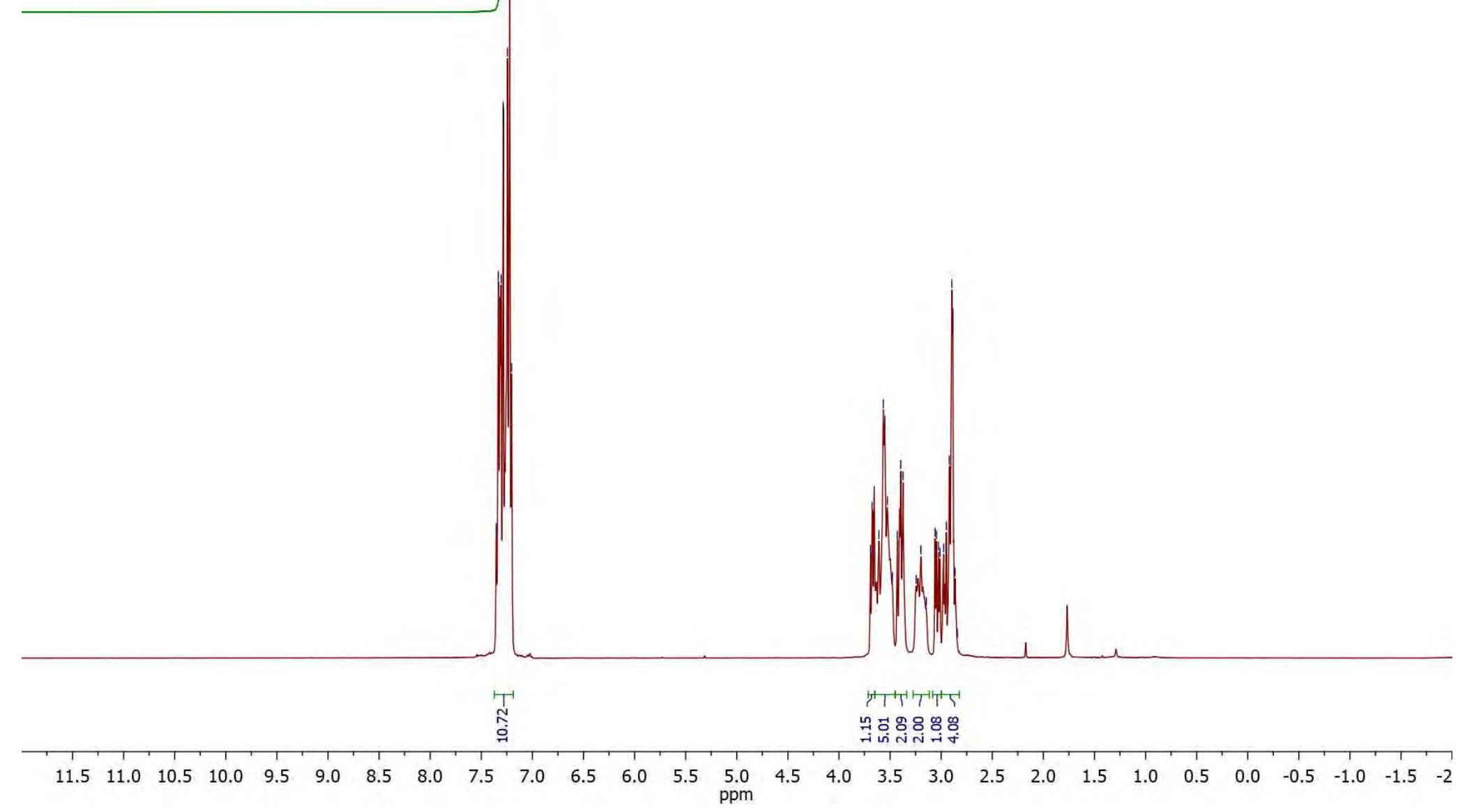


${ }^{13} \mathrm{C} \mathrm{NMR}, 100 \mathrm{MHz}, \mathrm{CDCl}_{3}, 25{ }^{\circ} \mathrm{C}$

|

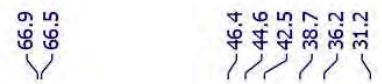<smiles>O=C(C(Cc1ccccc1)SCCc1ccccc1)N1CCOCC1</smiles>

4aa

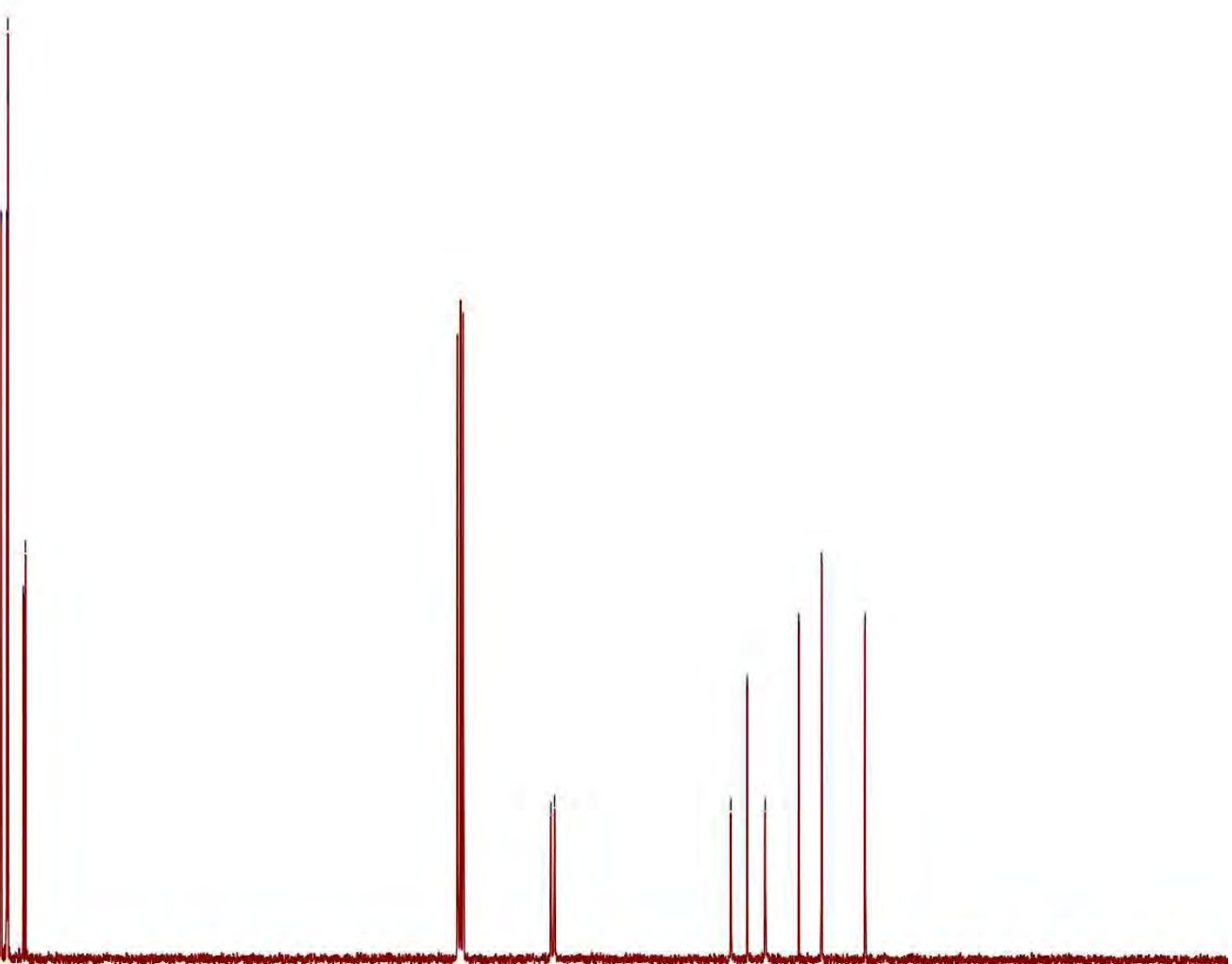

$0 \quad 200-190$ 
${ }^{1} \mathrm{H} \mathrm{NMR}, 400 \mathrm{MHz}, \mathrm{CDCl}_{3}, 25^{\circ} \mathrm{C}$

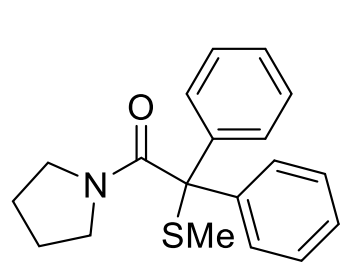

\section{กู่ ใุำ}

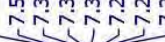

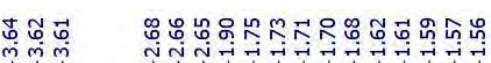

S4

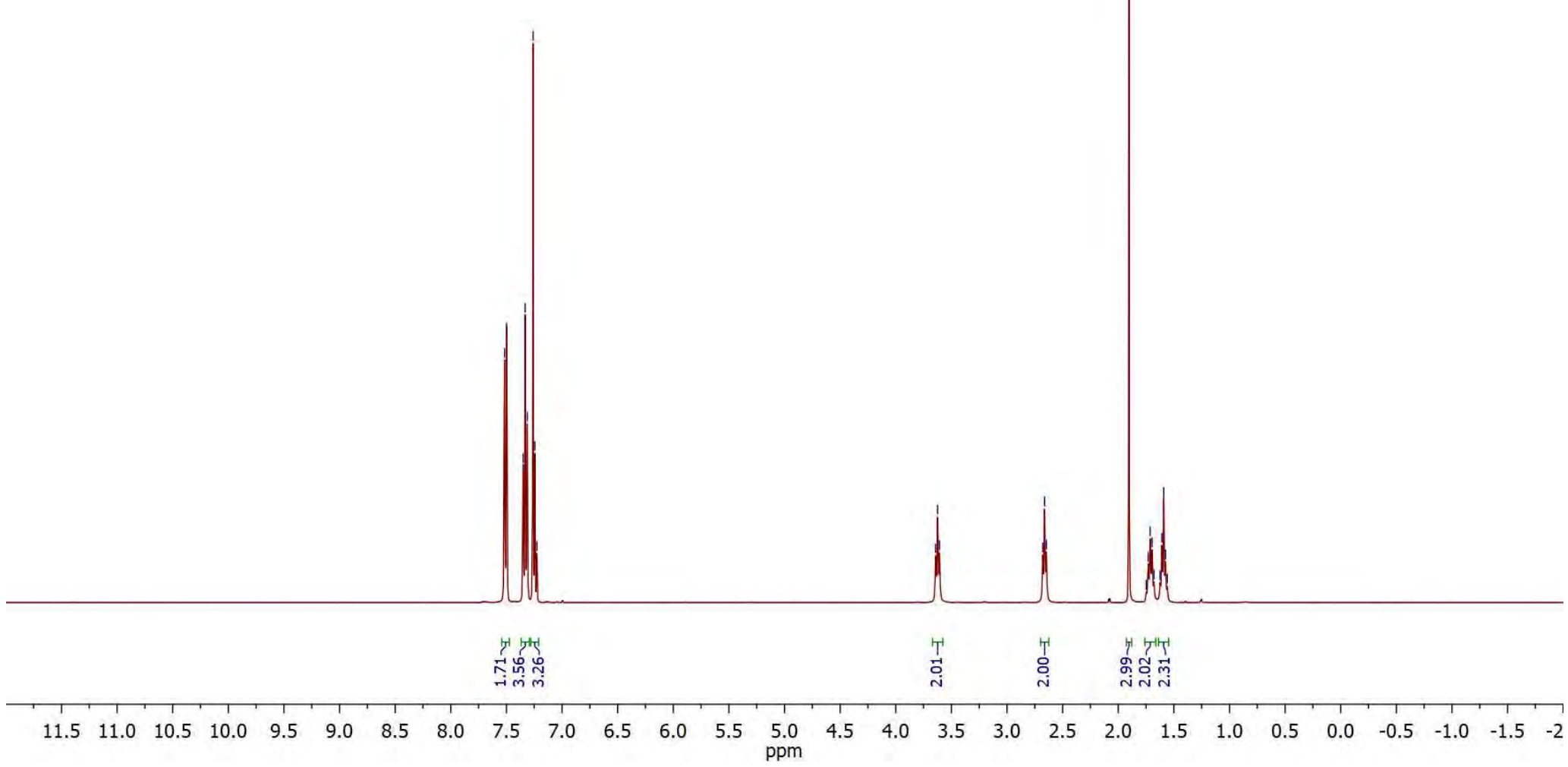


${ }^{13} \mathrm{C} \mathrm{NMR}, 100 \mathrm{MHz}, \mathrm{CDCl}_{3}, 25{ }^{\circ} \mathrm{C}$
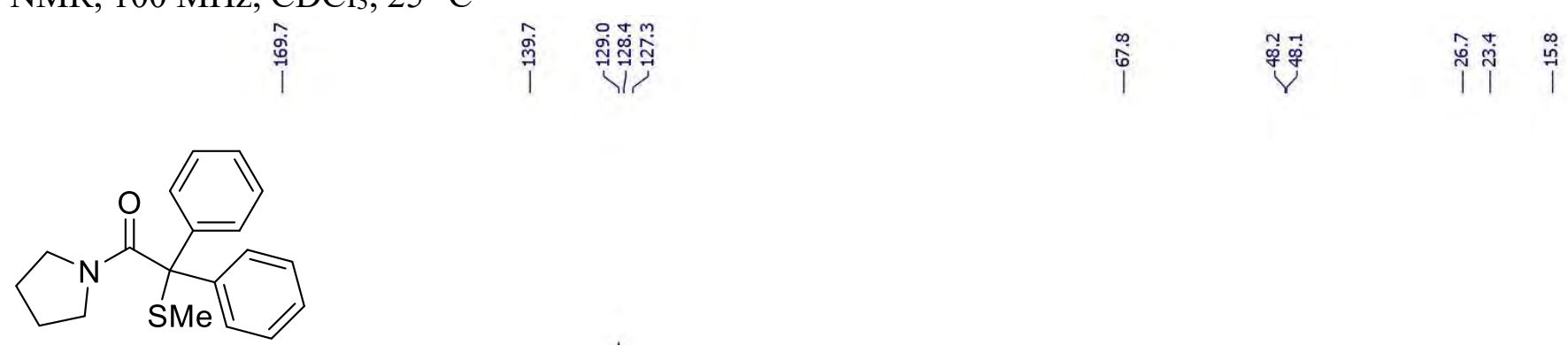

S4 
${ }^{13} \mathrm{C}$ NMR, $100 \mathrm{MHz}, \mathrm{CDCl}_{3}, 25^{\circ} \mathrm{C}$

$$
\begin{aligned}
& { }^{18} \mathrm{O} \\
& \mathrm{D}_{3} \mathrm{C}^{-\mathrm{S}_{-}} \mathrm{CD}_{3} \\
& 2 \mathrm{a}-{ }^{-18} \mathrm{O}-\boldsymbol{d}_{6}
\end{aligned}
$$

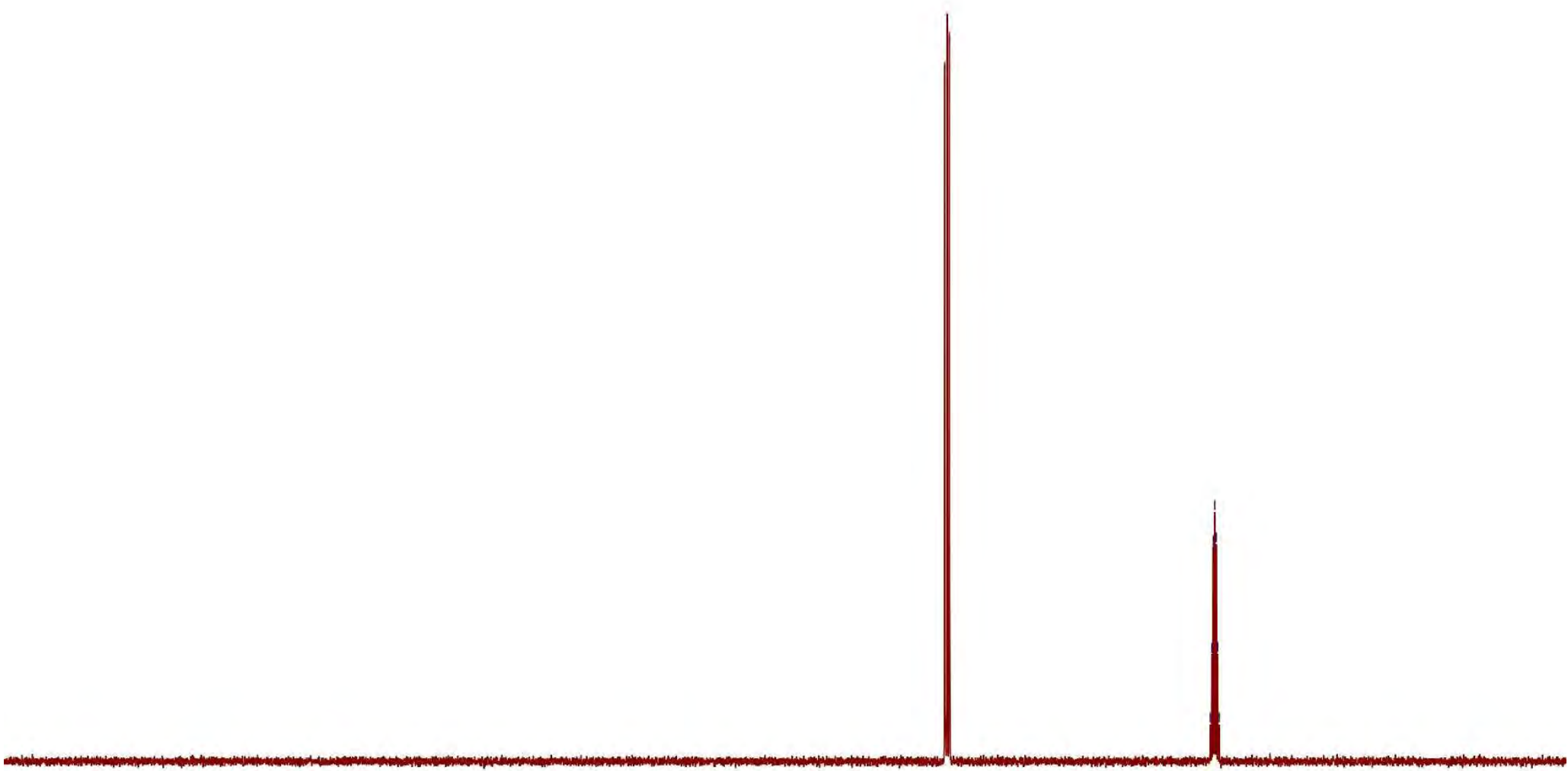

